University of Nebraska - Lincoln

DigitalCommons@University of Nebraska - Lincoln

Agronomy \& Horticulture -- Faculty Publications

Agronomy and Horticulture Department

$12-2004$

\title{
Sources of Plant Materials for Land Rehabilitation
}

\author{
Heidi Howard \\ Construction Engineering Research Laboratory \\ John A. Guretzky \\ University of Nebraska-Lincoln, jguretzky2@unl.edu \\ Brandy Koch \\ Construction Engineering Research Laboratory \\ Shannon McDowell \\ Construction Engineering Research Laboratory \\ Steven Warren \\ Construction Engineering Research Laboratory \\ See next page for additional authors
}

Follow this and additional works at: https://digitalcommons.unl.edu/agronomyfacpub

Part of the Plant Sciences Commons

Howard, Heidi; Guretzky, John A.; Koch, Brandy; McDowell, Shannon; Warren, Steven; and Howard, Gwyn L., "Sources of Plant Materials for Land Rehabilitation" (2004). Agronomy \& Horticulture -- Faculty Publications. 581.

https://digitalcommons.unl.edu/agronomyfacpub/581

This Article is brought to you for free and open access by the Agronomy and Horticulture Department at DigitalCommons@University of Nebraska - Lincoln. It has been accepted for inclusion in Agronomy \& Horticulture -Faculty Publications by an authorized administrator of DigitalCommons@University of Nebraska - Lincoln. 


\section{Authors}

Heidi Howard, John A. Guretzky, Brandy Koch, Shannon McDowell, Steven Warren, and Gwyn L. Howard 


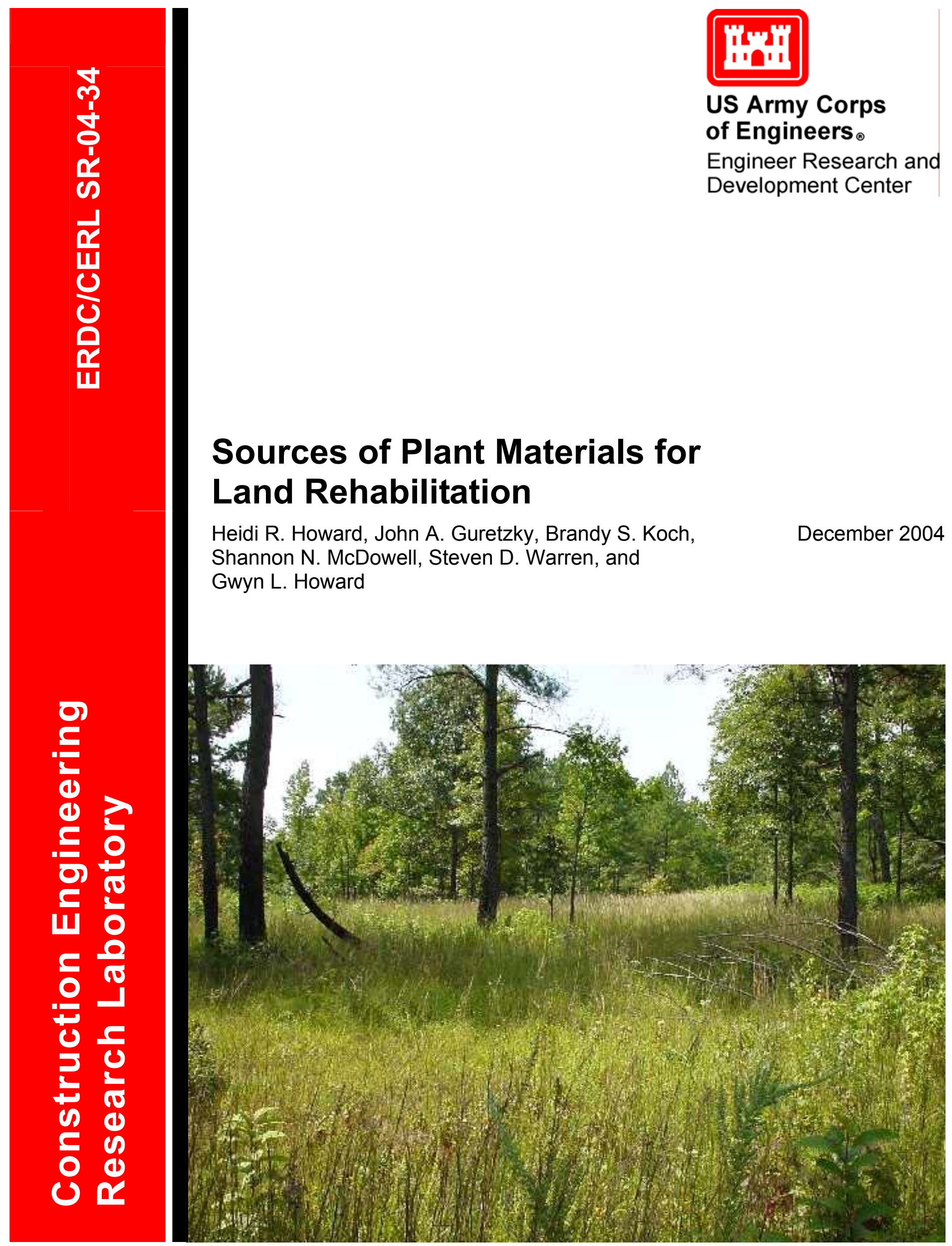





\section{Sources of Plant Materials for Land Rehabilitation}

Heidi R. Howard, John A. Guretzky, Brandy S. Koch, Shannon N. McDowell, Steven D. Warren, and Gwyn L. Howard

Construction Engineering Research Laboratory

PO Box 9005

Champaign, IL 61826-9005

Final Report

Approved for public release; distribution is unlimited.

Prepared for U.S. Army Corps of Engineers

Washington, DC 20314-1000

Under

Work Unit \#134GH5 
ABSTRACT: Military training often results in environmental degradation that has negative impacts on future training missions. The U.S. Army Construction Engineering Research Laboratory (CERL) conceptualized the Army's Integrated Training Area Management (ITAM) program in response to the concerns expressed by military land managers and trainers over the declining condition of training and testing lands. ITAM has evolved to include land condition and trend analysis (LCTA), environmental awareness (EA), training requirements integration (TRI), and land rehabilitation and maintenance (LRAM). The need for LRAM has increased dramatically over the past decade. Among the many difficulties faced in planning and implementing a land rehabilitation project is procurement of appropriate plant materials for revegetation of damaged lands. To satisfy government procurement requirements and ensure a fair and reasonable price for materials, it is usually necessary to identify and solicit bids from multiple vendors. Moreover, there is an increasing emphasis on the use of locally endemic or adapted plant species. These factors increase the demand to identify multiple commercial sources of regional or local sources of plant materials. This research compiled a comprehensive, current list of plant material vendors, including vendors of trees, shrubs, grasses, wild-flowers, and wetland plants.

DISCLAIMER: The contents of this report are not to be used for advertising, publication, or promotional purposes.

Citation of trade names does not constitute an official endorsement or approval of the use of such commercial products.

All product names and trademarks cited are the property of their respective owners. The findings of this report are not to be construed as an official Department of the Army position unless so designated by other authorized documents.

DESTROY THIS REPORT WHEN IT IS NO LONGER NEEDED. DO NOT RETURN IT TO THE ORIGINATOR. 


\section{Contents}

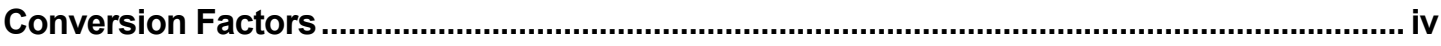

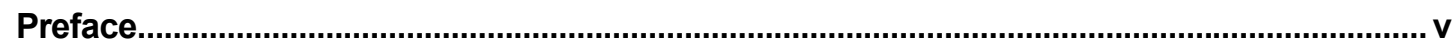

1 Introduction

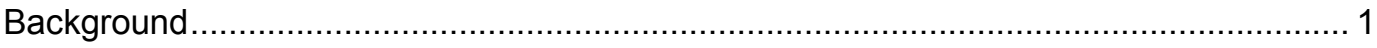

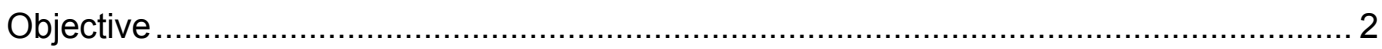

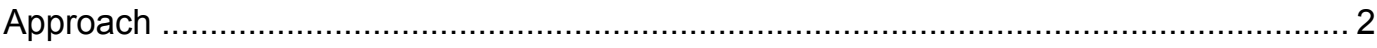

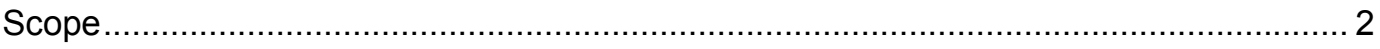

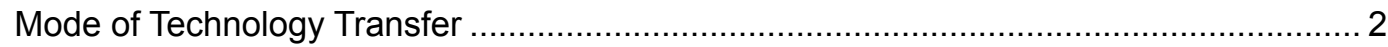

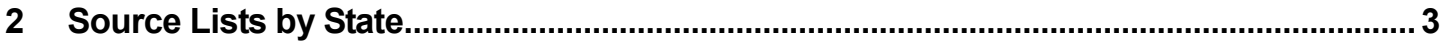

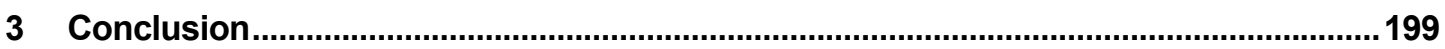

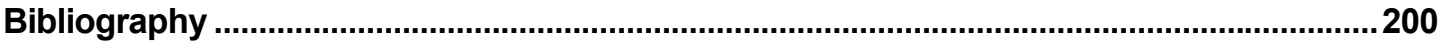

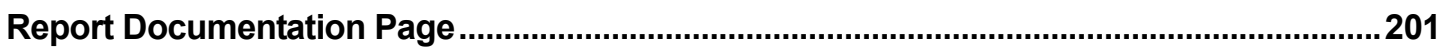




\section{Conversion Factors}

Non-SI* units of measurement used in this report can be converted to SI units as follows:

\begin{tabular}{|c|c|c|}
\hline Multiply & By & To Obtain \\
\hline acres & $4,046.873$ & square meters \\
\hline cubic feet & 0.02831685 & cubic meters \\
\hline cubic inches & 0.00001638706 & cubic meters \\
\hline degrees (angle) & 0.01745329 & radians \\
\hline degrees Fahrenheit & $(5 / 9) \times\left({ }^{\circ} \mathrm{F}-32\right)$ & degrees Celsius \\
\hline degrees Fahrenheit & $(5 / 9) \times\left({ }^{\circ} \mathrm{F}-32\right)+273.15$ & kelvins \\
\hline feet & 0.3048 & meters \\
\hline gallons (U.S. liquid) & 0.003785412 & cubic meters \\
\hline horsepower (550 ft-lb force per second) & 745.6999 & watts \\
\hline inches & 0.0254 & meters \\
\hline kips per square foot & 47.88026 & kilopascals \\
\hline kips per square inch & 6.894757 & megapascals \\
\hline miles (U.S. statute) & 1.609347 & kilometers \\
\hline pounds (force) & 4.448222 & newtons \\
\hline pounds (force) per square inch & 0.006894757 & megapascals \\
\hline pounds (mass) & 0.4535924 & kilograms \\
\hline square feet & 0.09290304 & square meters \\
\hline square miles & $2,589,998$ & square meters \\
\hline tons (force) & $8,896.443$ & newtons \\
\hline tons (2,000 pounds, mass) & 907.1847 & kilograms \\
\hline yards & 0.9144 & meters \\
\hline
\end{tabular}

\footnotetext{
*Système International d'Unités (“International System of Measurement”), commonly known as the "metric system."
} 


\section{Preface}

The work was performed by the Ecological Processes Branch (CN-N) of the Installations Division (CN), Construction Engineering Research Laboratory (CERL). The CERL Principal Investigator was Heidi Howard. The technical editor was William J. Wolfe, Information Technology Laboratory. Stephen E. Hodapp is Chief, CEERDCN-N, and Dr. John T. Bandy is Chief, CEERD-CN. The associated Technical Director was Dr. William D. Severinghaus, CEERD-CV-T. The Director of CERL is Dr. Alan W. Moore.

CERL is an element of the U.S. Army Engineer Research and Development Center (ERDC), U.S. Army Corps of Engineers. The Commander and Executive Director of ERDC is COL James R. Rowan, and the Director of ERDC is Dr. James R. Houston. 



\section{Introduction}

\section{Background}

War, by its very nature, is destructive to the environment. It follows logically that training for war is also destructive to the environment. Environmental ills associated with heavy training include soil compaction, soil erosion, siltation of waterways and wetlands, increased threat of flooding, loss of wildlife habitat, declining biodiversity, and invasion by noxious weeds. Such environmental degradation has a negative impact on the training mission as well. Tactical concealment resources are lost. Realism of the natural environment is diminished. Heavily eroded areas become dangerous or impassable with tactical vehicles. Moreover, public outcry over the condition of military training lands jeopardizes the image of the Armed Forces as a capable steward of the land.

The U.S. Army Construction Engineering Research Laboratory (CERL) conceptualized the Army's Integrated Training Area Management (ITAM) program in response to the concerns expressed by military land managers and trainers over the declining condition of training and testing lands,. ITAM has evolved to include land condition and trend analysis (LCTA), environmental awareness (EA), training requirements integration (TRI), and land rehabilitation and maintenance (LRAM).

The need for and the interest in LRAM have increased dramatically over the past decade. Among the many difficulties faced in planning and implementing a land rehabilitation project is procurement of appropriate plant materials for revegetation of damaged lands. To satisfy government procurement requirements and to ensure a fair and reasonable price for materials, it is usually necessary to identify and solicit bids from multiple vendors. Moreover, there is an increasing emphasis on the use of locally endemic or adapted plant species. These factors have increased the demand to identify multiple commercial sources of regional or local sources of plant materials. This work was undertaken to fill the need for a comprehensive, current listing of plant material vendors. 


\title{
Objective
}

The objective of this research was to create a comprehensive and up-to-date list of plant material vendors. The list includes vendors of trees, shrubs, grasses, wildflowers, and wetland plants.

\section{Approach}

1. Researchers began this work by purchasing a vendor list from a company that specializes in extracting and compiling business information from telephone directories.

2. Web-searches on the key phrase "seeds and bulbs retail" identified approximately 3650 businesses across the United States.

3. These lists were supplemented with information from a number of published sources (listed in the "Bibliography" section of this report).

4. Researchers called each listed business to verify that it was currently still active, and also to eliminate those business that sell only crop seed.

5. The resulting list was cross-referenced with the PMSource plant vendor database maintained by the Soil Conservation Service. Duplicate names were removed.

New names added from the PMSource were called to verify that they were still in business.

\section{Scope}

Users of this list of sources should be aware that not all vendors will carry all species. For any given species, it may be necessary to contact a large number of potential vendors. In addition, the availability and price of many species tend to vary seasonally and annually based on growing conditions and supply and demand.

\section{Mode of Technology Transfer}

This report will be made accessible through the World Wide Web (WWW) at URL:

\author{
http://www.cecer.army.mil
}




\section{Source Lists by State}

\section{Company Data by State}

\section{Alaska}

Anchorage

Alaska DNR Division of Forestry

550 W. Seventh Ave., Suite 1450

Anchorage, AK

99501

PHONE: (907) 269-8474

$\square$ SHRUB

$\square$ FORBS

v LOCAL TREE

$\square$ WETLAND

$\square$ LOCAL SHRUB

$\square$ LOCAL GRASS

URL: hittp://Www.dnr.state.ak.usfforestryl

Alaska Mill \& Seed

PO Box 101246. 114 N Orca St.

Anchorage, AK

99501

PHONE: (907) 279-4519

$\begin{array}{ll}\square \text { TREE } & \square \text { LOCAL TREE } \\ \square \text { SHRUB } & \square \text { LOCAL SHRUB } \\ \nabla \text { GRASS } & \nabla \text { LOCAL GRASS } \\ \square \text { FORBS } & \square \text { LOCAL FORBS } \\ \square \text { WETLAND } & \square \text { LOCAL WETLAND }\end{array}$

URL: none currently available

\section{Delta Junction}

Granite Mountain Farms

PO Box 1656

Delta Junction. AK

99737

PHONE: (907) 378-4526

$\begin{array}{ll}\square \text { TREE } & \square \text { LOCAL TREE } \\ \square \text { SHRUB } & \square \text { LOCAL SHRUB } \\ \nabla \text { GRASS } & \boldsymbol{v} \text { LOCAL GRASS } \\ \square \text { FORBS } & \square \text { LOCAL FORBS } \\ \square \text { WETLAND } & \square \text { LOCAL WETLAND }\end{array}$

URL: none currently available

\section{Ward Farms}

PO Box 1087, Mi 5 Sawmill Creek Rd.

Delta Junction. AK

99737

PHONE: (907) 895-5415

$\begin{array}{ll}\square \text { TREE } & \square \text { LOCAL TREE } \\ \square \text { SHRUB } & \square \text { LOCAL SHRUB } \\ \square \text { GRASS } & \checkmark \text { LOCAL GRASS } \\ \square \text { FORBS } & \square \text { LOCAL FORBS } \\ \square \text { WETLAND } & \square \text { LOCAL WETLAND }\end{array}$

URL: none currently available

Homer

Fritz Creek Gardens

PO Box 15226

Homer, AK

99603

PHONE: (907) 235-4969

$\begin{array}{ll}\square \text { TREE } & \square \text { LOCAL TREE } \\ \square \text { SHRUB } & \square \text { LOCAL SHRUB } \\ \square \text { GRASS } & \square \text { LOCAL GRASS } \\ \square \text { FORBS } & \square \text { LOCAL FORBS } \\ \square \text { WETLAND } & \square \text { LOCAL WETLAND }\end{array}$

URL: wnww.alaskahardy.com

Kenai

Seeds of Alaska

Box 3127, 48535 Cardwell Rd.

Kenai, AK

99611

PHONE: (907) 260-1980

$\begin{array}{ll}\square \text { TREE } & \square \text { LOCAL TREE } \\ \square \text { SHRUB } & \square \text { LOCAL SHRUB } \\ \nabla \text { GRASS } & \nabla \text { LOCAL GRASS } \\ \square \text { FORBS } & \square \text { LOCAL FORBS } \\ \square \text { WETLAND } & \square \text { LOCAL WETLAND }\end{array}$

URL: none currently available 
Palmer

Far North Tree and Seed Co.

PO Box 531, 5150 Gina Dr.

Palmer, AK

99645

PHONE: (907) 745-4024

$\begin{array}{ll}\nabla \text { TREE } & \square \text { LOCAL TREE } \\ \nabla \text { SHRUB } & \square \text { LOCAL SHRUB } \\ \square \text { GRASS } & \square \text { LOCAL GRASS } \\ \square \text { FORBS } & \square \text { LOCAL FORBS } \\ \square \text { WETLAND } & \square \text { LOCAL WETLAND }\end{array}$

URL: none currently available

Northern Native Seeds

Box 775

Palmer, AK

99645

PHONE: (907) 745-3773

$\begin{array}{ll}\square \text { TREE } & \square \text { LOCAL TREE } \\ \square \text { SHRUB } & \square \text { LOCAL SHRUB } \\ \nabla \text { GRASS } & \square \text { LOCAL GRASS } \\ \square \text { FORBS } & \square \text { LOCAL FORBS } \\ \square \text { WETLAND } & \square \text { LOCAL WETLAND }\end{array}$

URL: none currently available

Trapper Creek Farm/Alaska

PO Box 4272

$\checkmark$ TREE $\quad \nabla$ LOCAL TREE

Palmer, AK

99645

$\square$ SHRUB

$\checkmark$ GRASS

$\square$ LOCAL SHRUB

PHONE: (907) 745-1193

$\square$ FORBS $\square$ LOCAL FORBS

$\square$ WETLAND $\square$ LOCAL WETLAND

URL: none currently available

\section{Petersburg}

Wildwood Nursery

P.O.Box 1508

Petersburg, AK

99833

PHONE: (907) 772-4855

$\begin{array}{ll}\square \text { TREE } & \square \text { LOCAL TREE } \\ \square \text { SHRUB } & \square \text { LOCAL SHRUB } \\ \square \text { GRASS } & \square \text { LOCAL GRASS } \\ \square \text { FORBS } & \square \text { LOCAL FORBS } \\ \square \text { WETLAND } & \square \text { LOCAL WETLAND }\end{array}$

URL: none currently available

\section{Alabama}

Auburn

Alabama Crop Improvement Assoc.

PO Box 2619

$\square$ TREE $\quad \square$ LOCAL TREE

Auburn, AL

$\square$ SHRUB

$\checkmark$ GRASS

$\square$ LOCAL SHRUB

36831

$\square$ FORBS

$\nabla$ LOCAL GRASS

PHONE: (334) 821-7400

$\square$ WETLAND $\square$ LOCAL WETLAND

URL: www.ag.auburn.edu/ssca

\section{Camden}

Lambert Seed and Supply, Inc.

Box 128

Camden, AL

36726

PHONE: (205) 682-4111

$\begin{array}{ll}\square \text { TREE } & \square \text { LOCAL TREE } \\ \square \text { SHRUB } & \square \text { LOCAL SHRUB } \\ \square \text { GRASS } & \square \text { LOCAL GRASS } \\ \square \text { FORBS } & \square \text { LOCAL FORBS } \\ \square \text { WETLAND } & \square \text { LOCAL WETLAND }\end{array}$

URL: none currently available 
Cullman

Cullman Seed \& Feed Co., Inc.

Box 548

Cullman, AL

35055

PHONE: (205) 739-1966

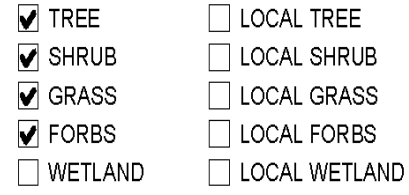

URL: none currently available

Elberta

Molpus Timberlands

29650 Cornstock Rd.

$\nabla$ TREE $\quad \nabla$ LOCAL TREE

Elberta, AL

36530

$\nabla$ SHRUB $\quad \nabla$ LOCAL SHRUB

PHONE: (251) 986-5210

$\checkmark$ GRASS $\nabla$ LOCAL GRASS

$\square$ FORBS $\square$ LOCAL FORBS

$\square$ WETLAND $\square$ LOCAL WETLAND

URL: www.molpus.com

Joppa

North Alabama Nursery Co.

P.O. Box 67

Joppa, AL

35087

PHONE: (256) 586-5676

$\begin{array}{ll}\nabla \text { TREE } & \square \text { LOCAL TREE } \\ \square \text { SHRUB } & \square \text { LOCAL SHRUB } \\ \square \text { GRASS } & \square \text { LOCAL GRASS } \\ \square \text { FORBS } & \square \text { LOCAL FORBS } \\ \square \text { WETLAND } & \square \text { LOCAL WETLAND }\end{array}$

URL: none currently available

Meridianville

Byers Nursery $\mathrm{C}_{0 .}$, Inc.

P.O. Box 560

$\checkmark$ TREE $\quad \nabla$ LOCAL TREE

Meridianville, AL

$\checkmark$ SHRUB

$\checkmark$ LOCAL SHRUB

35759

$\square$ GRASS

$\square$ LOCAL GRASS

PHONE: (256) 859-0690

$\square$ FORBS $\square$ LOCAL FORBS

$\square$ WETLAND $\square$ LOCAL WETLAND

URL: none currently available

Mobile

Flowerwood Nursery, Inc.

6470 Daupin Island Pkwy

Mobile, AL

36605

PHONE: (800) 862-4597

$\begin{array}{ll}\nabla \text { TREE } & \nabla \text { LOCAL TREE } \\ \nabla \text { SHRUB } & \square \text { LOCAL SHRUB } \\ \square \text { GRASS } & \square \text { LOCAL GRASS } \\ \square \text { FORBS } & \square \text { LOCAL FORBS } \\ \square \text { WETLAND } & \square \text { LOCAL WETLAND }\end{array}$

URL: http://Www.encoreazalea.com

\section{Montgomery}

Alabama Department of Conservation and Natural Resources

64 N. Union Street, Suite 468

Montgomery, AL

36130

PHONE: (334) 242-3486

$\square$ TREE

$\square$ SHRUB

$\square$ GRASS

$\square$ FORBS

$\square$ WETLAND $\square$ LOCAL TREE

$\square$ LOCAL SHRUB

$\square$ LOCAL GRASS

$\square$ LOCAL FORBS

$\square$ LOCAL WETLAND

URL: http://Www.denr.state.al.us/ 
Montgomery Seed \& Supply

1641 Federal Dr.

Montgomery, AL

36107

PHONE: (800) 633-8700

URL: none currently available

Montgomery Seed \& Supply

621 N. Decatur St.

Montgomery, AL

36104

PHONE: (334) 264-8051

URL: none currently available

$\begin{array}{ll}\square \text { TREE } & \square \text { LOCAL TREE } \\ \square \text { SHRUB } & \square \text { LOCAL SHRUB } \\ \square \text { GRASS } & \square \text { LOCAL GRASS } \\ \square \text { FORBS } & \square \text { LOCAL FORBS } \\ \square \text { WETLAND } & \square \text { LOCAL WETLAND }\end{array}$

$\begin{array}{ll}\square \text { TREE } & \square \text { LOCAL TREE } \\ \square \text { SHRUB } & \square \text { LOCAL SHRUB } \\ \square \text { GRASS } & \square \text { LOCAL GRASS } \\ \square \text { FORBS } & \square \text { LOCAL FORBS } \\ \square \text { WETLAND } & \square \text { LOCAL WETLAND }\end{array}$

Mountain Brook

South Pine, Inc.

4 Office Park Cir.

$\checkmark$ TREE $\quad \boldsymbol{v}$ LOCAL TREE

Mountain Brook, AL

35223

$\checkmark$ SHRUB $\forall$ LOCAL SHRUB

PHONE: (205) 879-1099

$\nabla$ GRASS $\quad \nabla$ LOCAL GRASS

$\checkmark$ FORBS $\checkmark$ LOCAL FORBS

$\checkmark$ WETLAND $\checkmark$ LOCAL WETLAND

URL: www.southpine.com

\section{Odenville}

International Forest Seed $\mathrm{C}_{0}$.

PO Box 490

Odenville, AL

35120

PHONE: (205) 629-6461

$\begin{array}{ll}\nabla \text { TREE } & \square \text { LOCAL TREE } \\ \square \text { SHRUB } & \square \text { LOCAL SHRUB } \\ \square \text { GRASS } & \square \text { LOCAL GRASS } \\ \square \text { FORBS } & \square \text { LOCAL FORBS } \\ \square \text { WETLAND } & \square \text { LOCAL WETLAND }\end{array}$

URL: none currently available

\section{Semmes}

Tom Dodd Nurseries, Inc.

9300 Wulff Rd. East, Drawer 45

Semmes, AL

36575

PHONE: (251) 649-1960

$\begin{array}{ll}\nabla \text { TREE } & \square \text { LOCAL TREE } \\ \nabla \text { SHRUB } & \nabla \text { LOCAL SHRUB } \\ \square \text { GRASS } & \square \text { LOCAL GRASS } \\ \nabla \text { FORBS } & \square \text { LOCAL FORBS } \\ \nabla \text { WETLAND } & \nabla \text { LOCAL WETLAND }\end{array}$

URL: www.tomdodd.com

Slocumb

Segrest Feed \& Seed Co

P.O. Box 339

Slocumb, AL

36375

PHONE: (334) 886-2371

$\begin{array}{ll}\square \text { TREE } & \square \text { LOCAL TREE } \\ \square \text { SHRUB } & \square \text { LOCAL SHRUB } \\ \square \text { GRASS } & \square \text { LOCAL GRASS } \\ \square \text { FORBS } & \square \text { LOCAL FORBS } \\ \square \text { WETLAND } & \square \text { LOCAL WETLAND }\end{array}$

URL: none currently available 
Wetumpka

Farmers Feed Svc.

5536 US Highway 23

Wetumpka, AL

36092

PHONE: (334) 567-6183

$\begin{array}{ll}\square \text { TREE } & \square \text { LOCAL TREE } \\ \square \text { SHRUB } & \square \text { LOCAL SHRUB } \\ \square \text { GRASS } & \square \text { LOCAL GRASS } \\ \square \text { FORBS } & \square \text { LOCAL FORBS } \\ \square \text { WETLAND } & \square \text { LOCAL WETLAND }\end{array}$

URL: none currently available

\section{Arkansas}

Ashdown

Kaufman Seeds, Inc.

34 E. Main St.

Ashdown, AR

71822

PHONE: (870) 898-3328

$\begin{array}{ll}\square \text { TREE } & \square \text { LOCAL TREE } \\ \square \text { SHRUB } & \square \text { LOCAL SHRUB } \\ \square \text { GRASS } & \square \text { LOCAL GRASS } \\ \square \text { FORBS } & \square \text { LOCAL FORBS } \\ \square \text { WETLAND } & \square \text { LOCAL WETLAND }\end{array}$

URL: none currently available

Elkins

Holland Wildflower Farm

2930 O'Neil Lane

Elkins, AR

72727

$\square$ SHRUB

$\nabla$ GRASS

PHONE: (479) 643-2622

$\checkmark$ FORBS $\quad \nabla$ LOCAL FORBS

$\square$ WETLAND $\square$ LOCAL WETLAND

URL: http:/Www hollandwildflowerfarm.com

\section{Hot Springs}

Weyerhaeuser $\mathrm{Co}$.

P.O. Box 1060

Hot Springs, AR

71902

PHONE: (800) 221-4898

$\begin{array}{ll}\square \text { TREE } & \square \text { LOCAL TREE } \\ \square \text { SHRUB } & \square \text { LOCAL SHRUB } \\ \square \text { GRASS } & \square \text { LOCAL GRASS } \\ \square \text { FORBS } & \square \text { LOCAL FORBS } \\ \square \text { WETLAND } & \square \text { LOCAL WETLAND }\end{array}$

URL: wWw.weyerhaeuser.com

\section{Little Rock}

Arkansas Forestry Commission

3821 West Roosevelt Rd

Little Rock, AR

72204

PHONE: (501) 296-1940

$\begin{array}{ll}\nabla \text { TREE } & \square \text { LOCAL TREE } \\ \square \text { SHRUB } & \square \text { LOCAL SHRUB } \\ \square \text { GRASS } & \square \text { LOCAL GRASS } \\ \square \text { FORBS } & \square \text { LOCAL FORBS } \\ \square \text { WETLAND } & \square \text { LOCAL WETLAND }\end{array}$

URL: http:/Www. forestry. state.ar.uSI

\section{London}

Pine Ridge Gardens

832 Sycamore Rd.

London, AR

72847

$\begin{array}{ll}\square \text { TREE } & \square \text { LOCAL TREE } \\ \square \text { SHRUB } & \square \text { LOCAL SHRUB } \\ \square \text { GRASS } & \square \text { LOCAL GRASS } \\ \square \text { FORBS } & \square \text { LOCAL FORBS } \\ \square \text { WETLAND } & \square \text { LOCAL WETLAND }\end{array}$

PHONE: (501) 293-4359

$\checkmark$ WETLAND

$\square$ LOCAL WETLAND

URL: none currently available 
Magnolia

Pittman Nursery Corp.

$\begin{array}{lll}\text { Box } 606 & \nabla \text { TREE } & \nabla \text { LOCAL TREE } \\ \text { Magnolia, AR } & \nabla \text { SHRUB } & \nabla \text { LOCAL SHRUB } \\ 71753 & \square \text { GRASS } & \nabla \text { LOCAL GRASS } \\ \text { PHONE: (800) 553-6661 } & \nabla \text { FORBS } & \nabla \text { LOCAL FORBS } \\ & \square \text { WETLAND } & \square \text { LOCAL WETLAND }\end{array}$

URL: none currently available

North Little Rock

Rivercity Seed $\mathrm{C}_{0}$.

P.O. Box 937

North Little Rock, AR

72115

PHONE: (501) 374-0302

$\begin{array}{ll}\square \text { TREE } & \square \text { LOCAL TREE } \\ \square \text { SHRUB } & \square \text { LOCAL SHRUB } \\ \square \text { GRASS } & \square \text { LOCAL GRASS } \\ \square \text { FORBS } & \square \text { LOCAL FORBS } \\ \square \text { WETLAND } & \square \text { LOCAL WETLAND }\end{array}$

URL: none currently available

\section{Sprindale}

Seed \& More

104 Hewitt St.

Sprindale, AR

72160

PHONE: (501) 751-8927

$\begin{array}{ll}\square \text { TREE } & \square \text { LOCAL TREE } \\ \square \text { SHRUB } & \square \text { LOCAL SHRUB } \\ \square \text { GRASS } & \square \text { LOCAL GRASS } \\ \square \text { FORBS } & \square \text { LOCAL FORBS } \\ \square \text { WETLAND } & \square \text { LOCAL WETLAND }\end{array}$

URL: none currently available

\section{Wynne}

Ridgecrest Nursery

3347 Hwy. 64

Wynne, AR

72396

PHONE: (501) 238-3763

$\begin{array}{ll}\nabla \text { TREE } & \checkmark \text { LOCAL TREE } \\ \nabla \text { SHRUB } & \checkmark \text { LOCAL SHRUB } \\ \nabla \text { GRASS } & \checkmark \text { LOCAL GRASS } \\ \nabla \text { FORBS } & \square \text { LOCAL FORBS } \\ \square \text { WETLAND } & \checkmark \text { LOCAL WETLAND }\end{array}$

URL: none currently available

\section{Arizona}

\section{Arizona City}

Arizona Plant Breeders

9343 W. Battaglia Dr.

Arizona City, AZ

85223

PHONE: (520) 466-6552

$\begin{array}{ll}\square \text { TREE } & \square \text { LOCAL TREE } \\ \square \text { SHRUB } & \square \text { LOCAL SHRUB } \\ \square \text { GRASS } & \square \text { LOCAL GRASS } \\ \square \text { FORBS } & \square \text { LOCAL FORBS } \\ \square \text { WETLAND } & \square \text { LOCAL WETLAND }\end{array}$

URL: none currently available

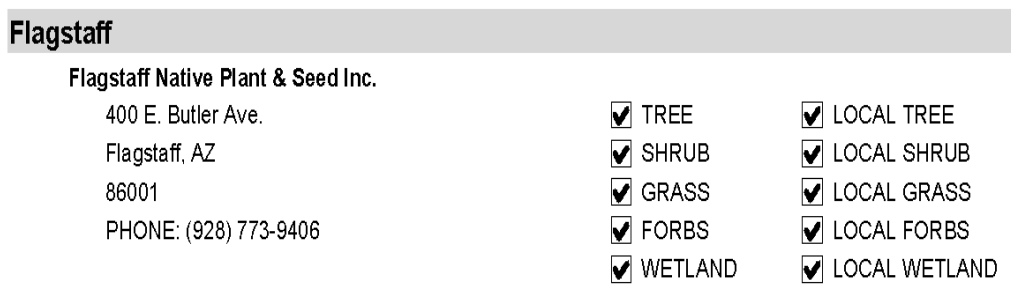

URL: www.nativeplantandseed.com 
Glendale

Mountain States Wholesale Nursery

10020 W. Glendale Ave

Glendale, AZ

85307

PHONE: (623) 247-8509

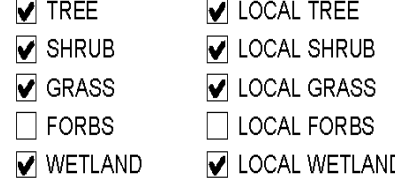

URL: www.mswn.com

Phoenix

Arizona State Land Department- Natural Resources 1616 W. Adams St.

Phoenix, AZ

85007

PHONE: (602) 542-4625

$\begin{array}{ll}\square \text { TREE } & \square \text { LOCAL TREE } \\ \square \text { SHRUB } & \square \text { LOCAL SHRUB } \\ \square \text { GRASS } & \square \text { LOCAL GRASS } \\ \square \text { FORBS } & \square \text { LOCAL FORBS } \\ \square \text { WETLAND } & \square \text { LOCAL WETLAND }\end{array}$

URL: http://Wwww.land. state.az.us/divisions/natural.htm

Queen Creek
$\begin{array}{lll}\text { Arid Zone Trees } \\ \text { Box } 167 & \square \text { TREE } & \square \text { LOCAL TREE } \\ \text { Queen Creek, AZ } & \square \text { SHRUB } & \square \text { LOCAL SHRUB } \\ 85242 & \square \text { GRASS } & \square \text { LOCAL GRASS } \\ \text { PHONE: (480) 987-9094 } & \square \text { FORBS } & \square \text { LOCAL FORBS } \\ & \square \text { WETLAND } & \square \text { LOCAL WETLAND }\end{array}$

URL: www.aridzonetrees.com

\begin{tabular}{|c|c|c|}
\hline \multicolumn{3}{|l|}{ Tempe } \\
\hline \multicolumn{3}{|l|}{ Wild Seed } \\
\hline Box 27751 & $\checkmark$ TREE & $\checkmark$ LOCAL TREE \\
\hline Tempe, AZ & $\nabla$ SHRUB & $\nabla$ LOCAL SHRUB \\
\hline 85285 & $\checkmark$ GRASS & $\checkmark$ LOCAL GRASS \\
\hline PHONE: (602) 276-3536 & $\checkmark$ FORBS & $\boldsymbol{\nabla}$ LOCAL FORBS \\
\hline & $\nabla$ WETLAND & $\checkmark$ LOCAL WETLAND \\
\hline
\end{tabular}

URL: none currently available

Tucson

Desert Trees Nursery

9559 N. Camino Del Plata

Tucson, AZ

85741

PHONE: (520) 297-5664

$\begin{array}{ll}\nabla \text { TREE } & \nabla \text { LOCAL TREE } \\ \nabla \text { SHRUB } & \square \text { LOCAL SHRUB } \\ \square \text { GRASS } & \square \text { LOCAL GRASS } \\ \square \text { FORBS } & \square \text { LOCAL FORBS } \\ \square \text { WETLAND } & \square \text { LOCAL WETLAND }\end{array}$

URL: none currently available

\section{Plant Materials Center}

3241 N. Romero Rd.

Tucson, AZ

85705

PHONE: (520) 670-6491

$\begin{array}{ll}\square \text { TREE } & \square \text { LOCAL TREE } \\ \square \text { SHRUB } & \square \text { LOCAL SHRUB } \\ \square \text { GRASS } & \square \text { LOCAL GRASS } \\ \square \text { FORBS } & \square \text { LOCAL FORBS } \\ \square \text { WETLAND } & \square \text { LOCAL WETLAND }\end{array}$

URL: none currently available 
Silverbell Nursery

2730 N. Silverbell Rd.

Tucson, AZ

85745

PHONE: (520) 622-3894

$\begin{array}{ll}\nabla \text { TREE } & \square \text { LOCAL TREE } \\ \nabla \text { SHRUB } & \square \text { LOCAL SHRUB } \\ \square \text { GRASS } & \square \text { LOCAL GRASS } \\ \square \text { FORBS } & \square \text { LOCAL FORBS } \\ \square \text { WETLAND } & \square \text { LOCAL WETLAND }\end{array}$

URL: none currently available

\section{California}

Aptos

Native Revival Nursery

8022 Soquel Dr

Aptos, CA

95003

PHONE: (831) 684-1811

$\begin{array}{ll}\square \text { TREE } & \square \text { LOCAL TREE } \\ \square \text { SHRUB } & \square \text { LOCAL SHRUB } \\ \nabla \text { GRASS } & \square \text { LOCAL GRASS } \\ \square \text { FORBS } & \square \text { LOCAL FORBS } \\ \square \text { WETLAND } & \square \text { LOCAL WETLAND }\end{array}$

URL: www.nativerevival.com

\section{Arroyo Grande}

Native Sons Wholesale Nursery

379 W. El Campo Rd.

Arroyo Grande $\mathrm{CA}$

93420

PHONE: (805) 481-5996

$\begin{array}{ll}\nabla \text { TREE } & \square \text { LOCAL TREE } \\ \square \text { SHRUB } & \square \text { LOCAL SHRUB } \\ \square \text { GRASS } & \square \text { LOCAL GRASS } \\ \square \text { FORBS } & \square \text { LOCAL FORBS } \\ \square \text { WETLAND } & \square \text { LOCAL WETLAND }\end{array}$

URL: www.nativeson.com

\section{Atascadero}

Bay Laurel Garden Center

2500 El Camino Real

Atascadero, CA

93422

PHONE: (805) 466-3449

$\begin{array}{ll}\nabla \text { TREE } & \square \text { LOCAL TREE } \\ \nabla \text { SHRUB } & \square \text { LOCAL SHRUB } \\ \nabla \text { GRASS } & \square \text { LOCAL GRASS } \\ \nabla \text { FORBS } & \square \text { LOCAL FORBS } \\ \square \text { WETLAND } & \square \text { LOCAL WETLAND }\end{array}$

URL: www.baylaurelnursery.com

Bolinas

Larner Seeds

Box 407235 Fern Rd.

Bolinas, CA

94924

PHONE: (415) 868-9407

$\begin{array}{ll}\nabla \text { TREE } & \square \text { LOCAL TREE } \\ \nabla \text { SHRUB } & \square \text { LOCAL SHRUB } \\ \nabla \text { GRASS } & \square \text { LOCAL GRASS } \\ \nabla \text { FORBS } & \square \text { LOCAL FORBS } \\ \square \text { WETLAND } & \square \text { LOCAL WETLAND }\end{array}$

URL: www.larnerseeds.com

\section{Brawley}

Azcal

767 S. 5th St.

Brawley, CA

92227

PHONE: (760) 344-2191

$\begin{array}{ll}\square \text { TREE } & \square \text { LOCAL TREE } \\ \square \text { SHRUB } & \square \text { LOCAL SHRUB } \\ \square \text { GRASS } & \square \text { LOCAL GRASS } \\ \square \text { FORBS } & \square \text { LOCAL FORBS } \\ \square \text { WETLAND } & \square \text { LOCAL WETLAND }\end{array}$

URL: none currently available 
Campbell

Sierra Pacific Turf Supply

510 Salmar Ave.

Campbell, CA

95008

$\square$ LOCAL TREE

PHONE: (406) 374-4700

$\square$ SHRUB $\quad \square$ LOCAL SHRUB

$\nabla$ GRASS $\square$ LOCAL GRASS

$\nabla$ FORBS $\quad \nabla$ LOCAL FORBS

$\square$ WETLAND $\square$ LOCAL WETLAND

URL: none currently available

Carpinteria

S\&S Seeds

Box 1275

Carpinteria, CA

93013

PHONE: (805) 684-0436

$\begin{array}{ll}\square \text { TREE } & \square \text { LOCAL TREE } \\ \nabla \text { SHRUB } & \nabla \text { LOCAL SHRUB } \\ \nabla \text { GRASS } & \nabla \text { LOCAL GRASS } \\ \nabla \text { FORBS } & \nabla \text { LOCAL FORBS } \\ \square \text { WETLAND } & \square \text { LOCAL WETLAND }\end{array}$

URL: www.ssseeds.com

\section{Castro Valley}

Clyde Robin Seed Co., Inc.

P.O. Box 2366

Castro Valley, CA

94546

PHONE: (510) 785-0425

$\begin{array}{ll}\square \text { TREE } & \square \text { LOCAL TREE } \\ \square \text { SHRUB } & \square \text { LOCAL SHRUB } \\ \square \text { GRASS } & \square \text { LOCAL GRASS } \\ \square \text { FORBS } & \square \text { LOCAL FORBS } \\ \square \text { WETLAND } & \square \text { LOCAL WETLAND }\end{array}$

URL: www.dyderobin.com

Davis

California NRCS / USDA Lyng Service Center

430 G Street \#4164

Davis, CA

95616

$\begin{array}{ll}\square \text { TREE } & \square \text { LOCAL TREE } \\ \square \text { SHRUB } & \square \text { LOCAL SHRUB } \\ \square \text { GRASS } & \square \text { LOCAL GRASS } \\ \square \text { FORBS } & \square \text { LOCAL FORBS } \\ \square \text { WETLAND } & \square \text { LOCAL WETLAND }\end{array}$

PHONE:

$\square$ WETLAND $\square$ LOCAL WETLAND

URL: http:I/Nww'ca.nrcs. usda.gov/

\section{Elk Grove}

Cornflower Farms, Inc.

Box 896

Elk Grove, CA

95759

PHONE: (916) 689-1015

$\begin{array}{ll}\nabla \text { TREE } & \nabla \text { LOCAL TREE } \\ \nabla \text { SHRUB } & \nabla \text { LOCAL SHRUB } \\ \nabla \text { GRASS } & \nabla \text { LOCAL GRASS } \\ \nabla \text { FORBS } & \nabla \text { LOCAL FORBS } \\ \nabla \text { WETLAND } & \nabla \text { LOCAL WETLAND }\end{array}$

URL: www.cornflowerfarms.com

\section{Escondido}

Horace Anderson Seedsman

20253 Elfin Forest Rd.

Escondido, CA

92029

$\checkmark$ TREE

$\checkmark$ SHRUB

$\checkmark$ LOCAL TREE

$\checkmark$ GRASS

$\checkmark$ LOCAL SHRUB

PHONE: (760) 471-1464

$\square$ FORBS

$\checkmark$ LOCAL GRASS

$\checkmark$ WETLAND

$\square$ LOCAL FORBS

URL: www.seedcoseeds.com 
Eureka

Freshwater Farms/North Coast Native Seed Bank 5851 Myrtle Ave

Eureka, CA

95503

PHONE: (707) 444-8261

$\checkmark$ TREE $\quad \nabla$ LOCAL TREE

$\nabla$ SHRUB $\quad \nabla$ LOCAL SHRUB

$\checkmark$ GRASS $\quad \square$ LOCAL GRASS

$\nabla$ FORBS $\quad \nabla$ LOCAL FORBS

$\nabla$ WETLAND $\nabla$ LOCAL WETLAND

URL: www.freshwaterfarms.com

Fairfax

ODonnells Fairfax Nursery

1700 Sir Francis Drake Blvd

Fairfax, $C A$

94930

PHONE: (415) 453-0372

$\checkmark$ SHRUB $\square$ LOCAL SHRUB

$\nabla$ GRASS $\quad \nabla$ LOCAL GRASS

$\checkmark$ FORBS $\quad \nabla$ LOCAL FORBS

$\nabla$ WETLAND $\nabla$ LOCAL WETLAND

URL: none currently available

Fulton

California Flora Nursery

Box 3

Fulton, $\mathrm{CA}$

95439

PHONE: (707) 528-8813

$\begin{array}{ll}\square \text { TREE } & \square \text { LOCAL TREE } \\ \square \text { SHRUB } & \square \text { LOCAL SHRUB } \\ \square \text { GRASS } & \square \text { LOCAL GRASS } \\ \square \text { FORBS } & \square \text { LOCAL FORBS } \\ \square \text { WETLAND } & \square \text { LOCAL WETLAND }\end{array}$

URL: http:/Wwww.calfloranursery.com/

\section{Grass Valley}

Far West Bulb Farm

14499 Lower Colfax Rd

Grass Valley, CA

95945

PHONE: (530) 272-4775

$\begin{array}{ll}\square \text { TREE } & \square \text { LOCAL TREE } \\ \square \text { SHRUB } & \square \text { LOCAL SHRUB } \\ \square \text { GRASS } & \square \text { LOCAL GRASS } \\ \square \text { FORBS } & \square \text { LOCAL FORBS } \\ \square \text { WETLAND } & \square \text { LOCAL WETLAND }\end{array}$

URL: www.californianativebulbs.com

Peaceful Valley Farm Supply

P.O. Box 2209

Grass Valley, $C A$

95945

PHONE: (530) 272-4769

$\begin{array}{ll}\square \text { TREE } & \square \text { LOCAL TREE } \\ \square \text { SHRUB } & \square \text { LOCAL SHRUB } \\ \nabla \text { GRASS } & \nabla \text { LOCAL GRASS } \\ \nabla \text { FORBS } & \nabla \text { LOCAL FORBS } \\ \square \text { WETLAND } & \square \text { LOCAL WETLAND }\end{array}$

URL: www.groworganic.com

\section{Kenwood}

Wildwood farm

10300 Sonoma Hwy

Kenwood, CA

95452

PHONE: (707) 833-1161

$\begin{array}{ll}\square \text { TREE } & \square \text { LOCAL TREE } \\ \square \text { SHRUB } & \square \text { LOCAL SHRUB } \\ \square \text { GRASS } & \square \text { LOCAL GRASS } \\ \square \text { FORBS } & \square \text { LOCAL FORBS } \\ \square \text { WETLAND } & \square \text { LOCAL WETLAND }\end{array}$

URL: wWW.wildwoodmaples.com 


\begin{tabular}{|c|c|c|}
\hline \multicolumn{3}{|l|}{ La Tuna Canyon } \\
\hline \multicolumn{3}{|l|}{ Theodore Payne Foundation } \\
\hline 10459 Tuxford St. & $\nabla$ TREE & $\checkmark$ LOCAL TREE \\
\hline La Tuna Canyon, CA & $\checkmark$ SHRUB & $\nabla$ LOCAL SHRUB \\
\hline 91352 & $\checkmark$ GRASS & $\checkmark$ LOCAL GRASS \\
\hline PHONE: (818) 768-1802 & $\nabla$ FORBS & $\nabla$ LOCAL FORBS \\
\hline & $\nabla$ WETLAND & $\checkmark$ LOCAL WETLAND \\
\hline
\end{tabular}

URL: www.theodorepayne.org

$\begin{array}{lll}\text { Lemoore } & & \\ \begin{array}{ll}\text { Limas Country Seeds } \\ \text { 9626 22nd Ave. }\end{array} & \square \text { TREE } & \square \text { LOCAL TREE } \\ \text { Lemoore, CA } & \square \text { SHRUB } & \square \text { LOCAL SHRUB } \\ 93245 & \square \text { GRASS } & \square \text { LOCAL GRASS } \\ \text { PHONE: (209) 924-1800 } & \square \text { FORBS } & \square \text { LOCAL FORBS } \\ & \square \text { WETLAND } & \square \text { LOCAL WETLAND }\end{array}$

URL: none currently available

\begin{tabular}{|c|c|c|}
\hline \multicolumn{3}{|l|}{ Livermore } \\
\hline \multicolumn{3}{|l|}{ Pacific Coast Seed Inc. } \\
\hline 533 Hawthorne Place & $\square$ TREE & $\square$ LOCAL TREE \\
\hline Livermore, CA & $\square$ SHRUB & $\square$ LOCAL SHRUB \\
\hline 94551 & $\nabla$ GRASS & $\square$ LOCAL GRASS \\
\hline PHONE: (925) 373-4417 & $\checkmark$ FORBS & $\square$ LOCAL FORBS \\
\hline & $\square$ WETLAND & $\square$ LOCAL WETLAND \\
\hline
\end{tabular}

\section{Lockeford}

Plant Materials Center

Box 68

Lockeford, CA

95237

PHONE: (209) 727-5319

$\begin{array}{ll}\square \text { TREE } & \square \text { LOCAL TREE } \\ \square \text { SHRUB } & \square \text { LOCAL SHRUB } \\ \square \text { GRASS } & \square \text { LOCAL GRASS } \\ \square \text { FORBS } & \square \text { LOCAL FORBS } \\ \square \text { WETLAND } & \square \text { LOCAL WETLAND }\end{array}$

URL: none currently available

Lompoc

Environmental Seed Producers

Box 2709, 1851 W. Olive Ave

Lompoc, CA

93438

PHONE: (805) 735-8888

$\begin{array}{ll}\square \text { TREE } & \square \text { LOCAL TREE } \\ \square \text { SHRUB } & \square \text { LOCAL SHRUB } \\ \square \text { GRASS } & \square \text { LOCAL GRASS } \\ \nabla \text { FORBS } & \square \text { LOCAL FORBS } \\ \square \text { WETLAND } & \square \text { LOCAL WETLAND }\end{array}$

URL: www.espseeds.com

\begin{tabular}{|c|c|c|}
\hline Los Angeles & & \\
\hline Stover Seed Co. & & \\
\hline PO Box 21488 & $\checkmark$ TREE & $\checkmark$ LOCAL TREE \\
\hline Los Angeles, CA & $\bar{V}$ SHRUB & $\bar{\nabla}$ LOCAL SHRUB \\
\hline 90012 & $\nabla$ GRASS & $\nabla$ LOCAL GRASS \\
\hline PHONE: (213) 626-9668 & $\nabla$ FORBS & $\underline{v}$ LOCAL FORBS \\
\hline & $\nabla$ WETLAND & $\checkmark$ LOCAL WETLAND \\
\hline
\end{tabular}

URL: www.stoverseed.com 
Manteca

Comprath Seed Co.

205 Stockton St.

Manteca, CA

95337

PHONE: (209) 623-6242

$\begin{array}{ll}\square \text { TREE } & \square \text { LOCAL TREE } \\ \square \text { SHRUB } & \square \text { LOCAL SHRUB } \\ \square \text { GRASS } & \square \text { LOCAL GRASS } \\ \square \text { FORBS } & \square \text { LOCAL FORBS } \\ \square \text { WETLAND } & \square \text { LOCAL WETLAND }\end{array}$

URL: www.seedsolutions.com

Kamprath Seed Co.

205 Stockton St.

Manteca, CA

95337

PHONE: (800) 466-9959

$\begin{array}{ll}\square \text { TREE } & \square \text { LOCAL TREE } \\ \nabla \text { SHRUB } & \checkmark \text { LOCAL SHRUB } \\ \nabla \text { GRASS } & \nabla \text { LOCAL GRASS } \\ \nabla \text { FORBS } & \nabla \text { LOCAL FORBS } \\ \square \text { WETLAND } & \checkmark \text { LOCAL WETLAND }\end{array}$

URL: none currently available

\section{Moss Landing}

Elkhorn Native Plant Nursery

1957 B Salinas Rd., P.O. Box 270

Moss Landing. $C A$

95039

PHONE: (831) 763-1207

$\begin{array}{ll}\nabla \text { TREE } & \nabla \text { LOCAL TREE } \\ \nabla \text { SHRUB } & \nabla \text { LOCAL SHRUB } \\ \nabla \text { GRASS } & \square \text { LOCAL GRASS } \\ \nabla \text { FORBS } & \nabla \text { LOCAL FORBS } \\ \nabla \text { WETLAND } & \nabla \text { LOCAL WETLAND }\end{array}$

URL: www.elkhornnursery.com

Northridge

KSA Jojoba

19025 Parthenia St.

Northridge, CA

91324

PHONE: (818) 701-1534

$\begin{array}{ll}\square \text { TREE } & \square \text { LOCAL TREE } \\ \square \text { SHRUB } & \nabla \text { LOCAL SHRUB } \\ \square \text { GRASS } & \square \text { LOCAL GRASS } \\ \square \text { FORBS } & \square \text { LOCAL FORBS } \\ \square \text { WETLAND } & \square \text { LOCAL WETLAND }\end{array}$

URL: http:/Www.jojoba-ksa.com

Ojai

Ojai Valley Seeds

P.O. Box 543

Ojai, $C A$

93024

PHONE: (805) 646-7743

$\begin{array}{ll}\nabla \text { TREE } & \square \text { LOCAL TREE } \\ \square \text { SHRUB } & \square \text { LOCAL SHRUB } \\ \square \text { GRASS } & \square \text { LOCAL GRASS } \\ \square \text { FORBS } & \square \text { LOCAL FORBS } \\ \square \text { WETLAND } & \square \text { LOCAL WETLAND }\end{array}$

URL: www.ojaiseeds.com

\section{Orange}

Village Nurseries

1589 N. Main St.

Orange, $\mathrm{CA}$

92867

PHONE: (714) 279-3100

$\begin{array}{ll}\nabla \text { TREE } & \square \text { LOCAL TREE } \\ \nabla \text { SHRUB } & \checkmark \text { LOCAL SHRUB } \\ \nabla \text { GRASS } & \checkmark \text { LOCAL GRASS } \\ \nabla \text { FORBS } & \nabla \text { LOCAL FORBS } \\ \square \text { WETLAND } & \square \text { LOCAL WETLAND }\end{array}$

URL: www.villagenurseries.com 
Palm Desert

The Living Desert, Palo Verde Garden Center

47900 Portola Ave.

Palm Desert, CA

92260

PHONE: (760) 346-5694

$\begin{array}{ll}\nabla \text { TREE } & \nabla \text { LOCAL TREE } \\ \nabla \text { SHRUB } & \nabla \text { LOCAL SHRUB } \\ \nabla \text { GRASS } & \square \text { LOCAL GRASS } \\ \nabla \text { FORBS } & \square \text { LOCAL FORBS } \\ \square \text { WETLAND } & \square \text { LOCAL WETLAND }\end{array}$

URL: www.livingdesert.org

Petaluma

North Coast Native Nursery

P.O. Box 744

$\nabla$ TREE $\quad \nabla$ LOCAL TREE

Petaluma, CA

94953

PHONE: (707) 769-1213

$\checkmark$ SHRUB $\quad \nabla$ LOCAL SHRUB

$\nabla$ GRASS $\nabla$ LOCAL GRASS

$\checkmark$ FORBS $\quad \nabla$ LOCAL FORBS

$\nabla$ WETLAND $\quad \nabla$ LOCAL WETLAND

URL: www.northcoastnativenursery.com

Pacific Open-Space

Box 744

Petaluma, CA

94953

PHONE: (707) 769-1213

$\begin{array}{ll}\nabla \text { TREE } & \nabla \text { LOCAL TREE } \\ \nabla \text { SHRUB } & \nabla \text { LOCAL SHRUB } \\ \nabla \text { GRASS } & \nabla \text { LOCAL GRASS } \\ \nabla \text { FORBS } & \nabla \text { LOCAL FORBS } \\ \nabla \text { WETLAND } & \nabla \text { LOCAL WETLAND }\end{array}$

URL: www.northcoastnativenursery.com

\section{Phillipsville}

Sylvandale Gardens

77 Avenue of the Giants

Phillipsville, CA

95559

PHONE: (707) 923-3606

$\begin{array}{ll}\nabla \text { TREE } & \square \text { LOCAL TREE } \\ \nabla \text { SHRUB } & \square \text { LOCAL SHRUB } \\ \nabla \text { GRASS } & \square \text { LOCAL GRASS } \\ \nabla \text { FORBS } & \square \text { LOCAL FORBS } \\ \square \text { WETLAND } & \square \text { LOCAL WETLAND }\end{array}$

URL: none currently available

Philo

Wildflowers International, Inc.

967 Highway 128

Philo, CA

95466

PHONE: (707) 895-3500

$\begin{array}{ll}\square \text { TREE } & \square \text { LOCAL TREE } \\ \square \text { SHRUB } & \square \text { LOCAL SHRUB } \\ \square \text { GRASS } & \square \text { LOCAL GRASS } \\ \square \text { FORBS } & \square \text { LOCAL FORBS } \\ \square \text { WETLAND } & \square \text { LOCAL WETLAND }\end{array}$

URL: none currently available

\section{Placerville}

Forest Seeds of California

1100 Indian Hill Rd

Placerville, CA

95667

PHONE: (530) 621-1551

$\begin{array}{ll}\nabla \text { TREE } & \square \text { LOCAL TREE } \\ \nabla \text { SHRUB } & \square \text { LOCAL SHRUB } \\ \square \text { GRASS } & \square \text { LOCAL GRASS } \\ \nabla \text { FORBS } & \nabla \text { LOCAL FORBS } \\ \nabla \text { WETLAND } & \square \text { LOCAL WETLAND }\end{array}$

URL: none currently available 
San Juan Capistrano

Tree of Life Nursery

PO Bos 635

San Juan Capistrano, CA

92693

PHONE: (949) 728-0685

URL: www.treeoflifenursery.com

\section{San Martin}

Saratoga Horticultural Foundation

15815 Murphy Ave.

San Martin, CA

95046

PHONE: (408) 779-3303

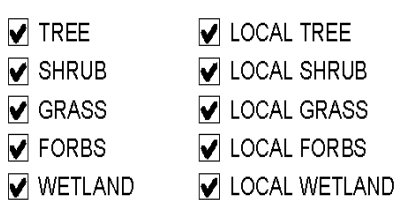

$\checkmark$ WETLAND $\checkmark$ LOCAL WETLAND

URL: www.saratogahortfoundation.org

Santa Margatrita

Las Pilitas Nursery

3232 Las Palitas Rd.

Santa Margatrita, CA

93453

PHONE: (805) 438-5992

$\begin{array}{ll}\square \text { TREE } & \square \text { LOCAL TREE } \\ \square \text { SHRUB } & \square \text { LOCAL SHRUB } \\ \square \text { GRASS } & \square \text { LOCAL GRASS } \\ \square \text { FORBS } & \square \text { LOCAL FORBS } \\ \square \text { WETLAND } & \square \text { LOCAL WETLAND }\end{array}$

WETLAND

URL: www.laspilitas.com

\section{Santa Rosa}

LeBallisters

1250 Sebastopol Rd

Santa Rosa, CA

95407

PHONE: (707) 526-6733

$\begin{array}{ll}\nabla \text { TREE } & \checkmark \text { LOCAL TREE } \\ \nabla \text { SHRUB } & \checkmark \text { LOCAL SHRUB } \\ \nabla \text { GRASS } & \nabla \text { LOCAL GRASS } \\ \nabla \text { FORBS } & \checkmark \text { LOCAL FORBS } \\ \nabla \text { WETLAND } & \checkmark \text { LOCAL WETLAND }\end{array}$

$\begin{array}{ll}\square \text { TREE } & \square \text { LOCAL TREE } \\ \nabla \text { SHRUB } & \square \text { LOCAL SHRUB } \\ \nabla \text { GRASS } & \square \text { LOCAL GRASS } \\ \nabla \text { FORBS } & \square \text { LOCAL FORBS } \\ \square \text { WETLAND } & \square \text { LOCAL WETLAND }\end{array}$

URL: www.leballistersseed com

Stockton

Lockhart Seeds Inc.

$3 N$. Wilson Way

Stockton, CA

95203

PHONE: (209) 466-4401

$\begin{array}{ll}\square \text { TREE } & \square \text { LOCAL TREE } \\ \square \text { SHRUB } & \square \text { LOCAL SHRUB } \\ \square \text { GRASS } & \square \text { LOCAL GRASS } \\ \square \text { FORBS } & \square \text { LOCAL FORBS } \\ \square \text { WETLAND } & \square \text { LOCAL WETLAND }\end{array}$

URL: none currently available

\section{Valley Springs}

Calaveras Nursery

1622 Hwy. 12

Valley Springs, CA

95252

PHONE: (209) 772-1823

$\begin{array}{ll}\square \text { TREE } & \square \text { LOCAL TREE } \\ \square \text { SHRUB } & \square \text { LOCAL SHRUB } \\ \square \text { GRASS } & \square \text { LOCAL GRASS } \\ \square \text { FORBS } & \square \text { LOCAL FORBS } \\ \square \text { WETLAND } & \square \text { LOCAL WETLAND }\end{array}$

URL: none currently available 
Vista

Carter Seeds

475 Mar Vista Dr.

Vista, CA

$\checkmark$ TREE

$\checkmark$ SHRUB

$\checkmark$ GRASS

$\nabla$ FORBS

$\checkmark$ LOCAL SHRUB

92083

PHONE: (800) 872-7711

$\square$ WETLAND $\square$ LOCAL WETLAND

URL: www.carterseeds.com

Wildomar

Agrono-Tec Seed Co.

21420 Bundy Cnyn Rd

Wildomar, CA

92595

$\square$ TREE

$\square$ SHRUB

$\square$ GRASS

$\square$ LOCAL TREE

PHONE: (909) 674-0638

$\square$ FORBS
$\square$ WETLAND

$\square$ LOCAL SHRUB

$\checkmark$ LOCAL GRASS

(9) 674-0638

$\square$ LOCAL FORBS

URL: none currently available

Windsor

Circuit Rider Productions

9619 Old Redwood Hwy

Windsor, CA

95492

PHONE: (707) 838-6641

$\begin{array}{ll}\square \text { TREE } & \square \text { LOCAL TREE } \\ \square \text { SHRUB } & \square \text { LOCAL SHRUB } \\ \square \text { GRASS } & \square \text { LOCAL GRASS } \\ \square \text { FORBS } & \square \text { LOCAL FORBS } \\ \square \text { WETLAND } & \square \text { LOCAL WETLAND }\end{array}$

URL: none currently available

\section{Woodside}

Yerba Buena Nursery

19500 Skyline Blvd

Woodside, CA

94062

PHONE: (650) 851-1668

$\begin{array}{ll}\nabla \text { TREE } & \square \text { LOCAL TREE } \\ \nabla \text { SHRUB } & \checkmark \text { LOCAL SHRUB } \\ \square \text { GRASS } & \checkmark \text { LOCAL GRASS } \\ \nabla \text { FORBS } & \square \text { LOCAL FORBS } \\ \square \text { WETLAND } & \square \text { LOCAL WETLAND }\end{array}$

URL: www.yerbabuenanursery.com

\section{Colorado}

Arvada

Applewood Seed Co.

5310 Vivian St.

Anvada, CO

80002

PHONE: (303) 431-7333

$\begin{array}{ll}\square \text { TREE } & \square \text { LOCAL TREE } \\ \square \text { SHRUB } & \square \text { LOCAL SHRUB } \\ \square \text { GRASS } & \square \text { LOCAL GRASS } \\ \nabla \text { FORBS } & \boldsymbol{\nabla} \text { LOCAL FORBS } \\ \square \text { WETLAND } & \square \text { LOCAL WETLAND }\end{array}$

URL: www.applewoodseed.com

\section{Brighton}

Little Valley Nurseries, Inc.

13022 E. 136th Ave.

Brighton, $C O$

80601

PHONE: (303) 659-6708

$\begin{array}{ll}\square \text { TREE } & \square \text { LOCAL TREE } \\ \square \text { SHRUB } & \square \text { LOCAL SHRUB } \\ \square \text { GRASS } & \square \text { LOCAL GRASS } \\ \square \text { FORBS } & \square \text { LOCAL FORBS } \\ \square \text { WETLAND } & \square \text { LOCAL WETLAND }\end{array}$

URL: www.lwwn.com 


\section{Coaldale}

Western Native Seed

PO Box 188

Coaldale, $\mathrm{CO}$

81222

PHONE: (719) 942-3935

URL: www.westernnativeseed.com

Denver

Arkansas Valley Seeds $\mathrm{C}_{0}$

4625 Colorado Blvd.

Denver, $C O$

80216

PHONE: (303) 320-7500

$\begin{array}{ll}\nabla \text { TREE } & \nabla \text { LOCAL TREE } \\ \nabla \text { SHRUB } & \nabla \text { LOCAL SHRUB } \\ \nabla \text { GRASS } & \nabla \text { LOCAL GRASS } \\ \nabla \text { FORBS } & \nabla \text { LOCAL FORBS } \\ \nabla \text { WETLAND } & \nabla \text { LOCAL WETLAND }\end{array}$

URL: www.seedsolutions.com

Colorado DNR

1313 Sherman St., Rm. 718

Denver, $C O$

80203

PHONE: (303) 866-3311

$\begin{array}{ll}\square \text { TREE } & \square \text { LOCAL TREE } \\ \square \text { SHRUB } & \square \text { LOCAL SHRUB } \\ \square \text { GRASS } & \square \text { LOCAL GRASS } \\ \square \text { FORBS } & \square \text { LOCAL FORBS } \\ \square \text { WETLAND } & \square \text { LOCAL WETLAND }\end{array}$

URL: http://Www dnr.state.co.us/

\section{Dolores}

Southwest Seed, Inc.

13260 Rd. 29

Dolores, CO

81323

PHONE: (970) 565-8722

$\begin{array}{ll}\square \text { TREE } & \square \text { LOCAL TREE } \\ \square \text { SHRUB } & \square \text { LOCAL SHRUB } \\ \square \text { GRASS } & \square \text { LOCAL GRASS } \\ \square \text { FORBS } & \square \text { LOCAL FORBS } \\ \square \text { WETLAND } & \square \text { LOCAL WETLAND }\end{array}$

URL: www.southwestseed.com

Dove Creek

Carhart Feed \& Seed, Inc.

Box 55

Dove Creek, CO

81324

PHONE: (970) 677-2233

$\begin{array}{ll}\square \text { TREE } & \square \text { LOCAL TREE } \\ \nabla \text { SHRUB } & \square \text { LOCAL SHRUB } \\ \square \text { GRASS } & \square \text { LOCAL GRASS } \\ \nabla \text { FORBS } & \square \text { LOCAL FORBS } \\ \square \text { WETLAND } & \square \text { LOCAL WETLAND }\end{array}$

URL: none currently available

\section{Fort Collins}

\section{Colorado State Forest Nursery}

Foothills Campus, Bldg. 1060"

Fort Collins, $\mathrm{CO}$

80523

PHONE: (970) 491-8429

$\begin{array}{ll}\square \text { TREE } & \square \text { LOCAL TREE } \\ \nabla \text { SHRUB } & \square \text { LOCAL SHRUB } \\ \nabla \text { GRASS } & \square \text { LOCAL GRASS } \\ \square \text { FORBS } & \square \text { LOCAL FORBS } \\ \square \text { WETLAND } & \square \text { LOCAL WETLAND }\end{array}$

URL: none currently available

$\begin{array}{ll}\nabla \text { TREE } & \square \text { LOCAL TREE } \\ \nabla \text { SHRUB } & \square \text { LOCAL SHRUB } \\ \square \text { GRASS } & \square \text { LOCAL GRASS } \\ \square \text { FORBS } & \square \text { LOCAL FORBS } \\ \square \text { WETLAND } & \square \text { LOCAL WETLAND }\end{array}$

$\square$ WETLAND $\square$ LOCAL WETLAND 
Franktown

Country Lane Wholesale Nursery

2979 N. Hwy. 83

Franktown, $\mathrm{CO}$

80116

PHONE: (303) 688-2442

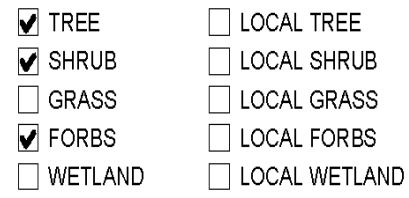

URL: none currently available

\section{Ft. Lupton}

Aquatic \& Wetland Co

9999 Weld County Rd. \#25

Ft. Lupton, CO

80621

PHONE: (303) 857-6157

$\begin{array}{ll}\square \text { TREE } & \square \text { LOCAL TREE } \\ \square \text { SHRUB } & \square \text { LOCAL SHRUB } \\ \square \text { GRASS } & \square \text { LOCAL GRASS } \\ \square \text { FORBS } & \square \text { LOCAL FORBS } \\ \square \text { WETLAND } & \square \text { LOCAL WETLAND }\end{array}$

URL: http://Www aquaticandwetland.com/

Golden

Green Acres Nursery

4990 McIntyre St

Golden, CO

80403

PHONE: (303) 279-8204

$\begin{array}{ll}\nabla \text { TREE } & \square \text { LOCAL TREE } \\ \nabla \text { SHRUB } & \nabla \text { LOCAL SHRUB } \\ \nabla \text { GRASS } & \nabla \text { LOCAL GRASS } \\ \nabla \text { FORBS } & \nabla \text { LOCAL FORBS } \\ \nabla \text { WETLAND } & \checkmark \text { LOCAL WETLAND }\end{array}$

URL: www.greenacresnursery.com

\section{Grand Junction}

Greenfields Seed

520 S. 9th St.

Grand Junction, CO

81505

PHONE: (970) 241-0979

$\begin{array}{ll}\square \text { TREE } & \square \text { LOCAL TREE } \\ \square \text { SHRUB } & \square \text { LOCAL SHRUB } \\ \square \text { GRASS } & \square \text { LOCAL GRASS } \\ \square \text { FORBS } & \square \text { LOCAL FORBS } \\ \square \text { WETLAND } & \square \text { LOCAL WETLAND }\end{array}$

URL: none currently available

\section{Greeley}

Pawnee Buttes Seed Inc.

P.O. Box 100

Greeley, CO

80632

PHONE: (970) 356-7002

$\begin{array}{ll}\square \text { TREE } & \square \text { LOCAL TREE } \\ \nabla \text { SHRUB } & \square \text { LOCAL SHRUB } \\ \nabla \text { GRASS } & \square \text { LOCAL GRASS } \\ \nabla \text { FORBS } & \square \text { LOCAL FORBS } \\ \nabla \text { WETLAND } & \square \text { LOCAL WETLAND }\end{array}$

URL: www.pawneebuttesseed.com

\section{Greely}

Sharp Bros. Seed Co.

$101 \mathrm{E}$. 4th St.

$\square$ TREE

Greely, CO

80631

PHONE: (970) 356-4710

$\checkmark$ SHRUB

$\square$ GRASS

$\square$ LOCAL TREE

$\checkmark$ FORBS

$\square$ LOCAL SHRUB

WETLAND

$\square$ LOCAL GRASS

$\square$ LOCAL FORBS

URL: www.sharpseed.com 
Jaroso

Dean Swift Seed Co.

$P O$ Box B

Jaroso, CO

81138

PHONE: (719) 672-3739

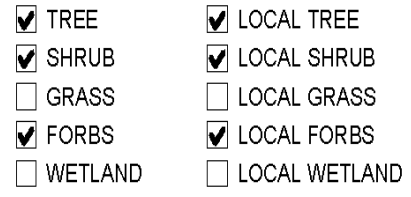

URL: www.deanswittseed.com

Longmont

Arkansas Valley Seeds $\mathrm{Co}$.

43333 Highway 66

Longmont, $\mathrm{CO}$

80504

$\begin{array}{ll}\square \text { TREE } & \square \text { LOCAL TREE } \\ \square \text { SHRUB } & \square \text { LOCAL SHRUB } \\ \square \text { GRASS } & \square \text { LOCAL GRASS } \\ \square \text { FORBS } & \square \text { LOCAL FORBS } \\ \square \text { WETLAND } & \square \text { LOCAL WETLAND }\end{array}$

PHONE: (303) 665-6642

$\square$ WETLAND $\square$ LOCAL WETLAND

URL: www.seedsolutions.com

\section{Rocky Ford}

Arkansas Valley Seeds, Inc.

PO Box 270

Rocky Ford, CO

81067

PHONE: (719) 254-7469

$\begin{array}{ll}\square \text { TREE } & \square \text { LOCAL TREE } \\ \square \text { SHRUB } & \square \text { LOCAL SHRUB } \\ \nabla \text { GRASS } & \square \text { LOCAL GRASS } \\ \square \text { FORBS } & \square \text { LOCAL FORBS } \\ \square \text { WETLAND } & \square \text { LOCAL WETLAND }\end{array}$

URL: none currently available

\section{Connecticut}

\section{Bolton}

Hope River Nursery

251 Hope River Rd

Boton, CT

06040

PHONE: (860) 646-7099

$\begin{array}{ll}\square \text { TREE } & \square \text { LOCAL TREE } \\ \square \text { SHRUB } & \square \text { LOCAL SHRUB } \\ \square \text { GRASS } & \square \text { LOCAL GRASS } \\ \square \text { FORBS } & \square \text { LOCAL FORBS } \\ \square \text { WETLAND } & \square \text { LOCAL WETLAND }\end{array}$

URL: none currently available

Treats Trees

87 Bolton Center Rd.

Bolton, CT

06043

PHONE: (860) 649-5184

$\begin{array}{ll}\nabla \text { TREE } & \square \text { LOCAL TREE } \\ \nabla \text { SHRUB } & \checkmark \text { LOCAL SHRUB } \\ \square \text { GRASS } & \square \text { LOCAL GRASS } \\ \nabla \text { FORBS } & \square \text { LOCAL FORBS } \\ \nabla \text { WETLAND } & \nabla \text { LOCAL WETLAND }\end{array}$

URL: none currently available

\section{Brookfield Center}

Haviland Farm Market

8 Cedar Hill Rd.

Brookfield Center, CT

06805

PHONE: (203) 775-1149

$\begin{array}{ll}\nabla \text { TREE } & \square \text { LOCAL TREE } \\ \square \text { SHRUB } & \square \text { LOCAL SHRUB } \\ \square \text { GRASS } & \square \text { LOCAL GRASS } \\ \square \text { FORBS } & \square \text { LOCAL FORBS } \\ \square \text { WETLAND } & \square \text { LOCAL WETLAND }\end{array}$

URL: none currently available 
Burlington

Merriman Tree Farm \& Nursery

455 Milford St.

Burlington, CT

06013

PHONE: (860) 675-3480

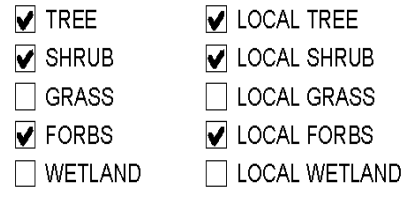

URL: none currently available

\section{Cheshire}

Cheshire Nursery, Inc

1317 S. Main St

Cheshire, CT

06410

PHONE: (203) 272-3228

$\begin{array}{ll}\square \text { TREE } & \square \text { LOCAL TREE } \\ \square \text { SHRUB } & \square \text { LOCAL SHRUB } \\ \square \text { GRASS } & \square \text { LOCAL GRASS } \\ \square \text { FORBS } & \square \text { LOCAL FORBS } \\ \square \text { WETLAND } & \square \text { LOCAL WETLAND }\end{array}$

URL: none currently available

Chesire

Casterano's Greenhouses and Farms, Inc.

$\begin{array}{lll}\text { 1030 S. Meriden Rd. } & \square \text { TREE } & \square \text { LOCAL TREE } \\ \text { Chesire, CT } & \square \text { SHRUB } & \square \text { LOCAL SHRUB } \\ 06410 & \square \text { GRASS } & \square \text { LOCAL GRASS } \\ \text { PHONE: (203) 272-6444 } & \square \text { FORBS } & \square \text { LOCAL FORBS } \\ & \square \text { WETLAND } & \square \text { LOCAL WETLAND }\end{array}$

URL: www.casteranosfarms.com

Clinton

Clinton Nurseries, Inc.

114 Main St

Clinton, CT

06776

$\square$ TREE $\quad \square$ LOCAL TREE

$\checkmark$ SHRUB

$\checkmark$ LOCAL SHRUB

PHONE: (860) 669-8611

$\square$ GRASS $\square$ LOCAL GRASS

$\square$ FORBS $\square$ LOCAL FORBS

$\square$ WETLAND $\square$ LOCAL WETLAND

URL: none currently available

\section{Collinsville}

Riverside Nursery

56 River Rd.

Collinsville, CT

06022

PHONE: (860) 693-2285

$\begin{array}{ll}\nabla \text { TREE } & \nabla \text { LOCAL TREE } \\ \nabla \text { SHRUB } & \square \text { LOCAL SHRUB } \\ \square \text { GRASS } & \square \text { LOCAL GRASS } \\ \nabla \text { FORBS } & \square \text { LOCAL FORBS } \\ \square \text { WETLAND } & \square \text { LOCAL WETLAND }\end{array}$

URL: http://Www riverside-nursery.com/

\section{Cromwell}

Millane Nurseries, Inc.

604 Main St.

Cromwell, CT

06416

PHONE: (860) 635-5500

$\begin{array}{ll}\square \text { TREE } & \square \text { LOCAL TREE } \\ \square \text { SHRUB } & \square \text { LOCAL SHRUB } \\ \square \text { GRASS } & \square \text { LOCAL GRASS } \\ \square \text { FORBS } & \square \text { LOCAL FORBS } \\ \square \text { WETLAND } & \square \text { LOCAL WETLAND }\end{array}$

URL: none currently available 
Deep River

Acer Gardens

447 Winthrop Rd.

Deep River, CT

06417

PHONE: (860) 526-9056

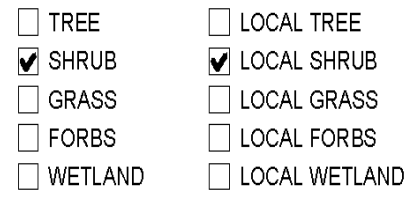

URL: none currently available

\section{East Haddam}

Balleks Garden Center

Maple Ave

$\nabla$ TREE $\quad \nabla$ LOCAL TREE

East Haddam, CT

06423

$\checkmark$ SHRUB

$\checkmark$ LOCAL SHRUB

PHONE: (860) 873-8878

$\square$ GRASS $\square$ LOCAL GRASS

$\checkmark$ FORBS $\quad \nabla$ LOCAL FORBS

$\square$ WETLAND $\square$ LOCAL WETLAND

URL: none currently available

\section{East Haven}

East Haven Landscape Products

10 Mill St.

East Haven, CT

06512

PHONE: (203) 467-6260

$\begin{array}{ll}\square \text { SHRUB } & \square \text { LOCAL SHRUB } \\ \square \text { GRASS } & \square \text { LOCAL GRASS } \\ \square \text { FORBS } & \square \text { LOCAL FORBS } \\ \square \text { WETLAND } & \square \text { LOCAL WETLAND }\end{array}$

URL: none currently available

\section{Greenwich}

Greenwich Landscaping $\mathrm{C}_{0}$

732 North St.

Greenwich, CT

06830

PHONE: (203) 869-1022

URL: none currently available

Shemin Nurseries

1081 King St.

Greenwich, CT

06830

PHONE: (203) 531-6700

URL: none currently available

\section{Guilford}

Hockings Garden Center

1200 Durham Rd.

Guilford, CT

06484

PHONE: (203) 458-2518

$\begin{array}{ll}\square \text { TREE } & \square \text { LOCAL TREE } \\ \square \text { SHRUB } & \square \text { LOCAL SHRUB } \\ \square \text { GRASS } & \square \text { LOCAL GRASS } \\ \square \text { FORBS } & \square \text { LOCAL FORBS } \\ \square \text { WETLAND } & \square \text { LOCAL WETLAND }\end{array}$

URL: none currently available

$\begin{array}{ll}\nabla \text { TREE } & \square \text { LOCAL TREE } \\ \nabla \text { SHRUB } & \square \text { LOCAL SHRUB } \\ \square \text { GRASS } & \square \text { LOCAL GRASS } \\ \nabla \text { FORBS } & \square \text { LOCAL FORBS } \\ \square \text { WETLAND } & \square \text { LOCAL WETLAND }\end{array}$

$\begin{array}{ll}\nabla \text { TREE } & \square \text { LOCAL TREE } \\ \square \text { SHRUB } & \square \text { LOCAL SHRUB } \\ \square \text { GRASS } & \square \text { LOCAL GRASS } \\ \square \text { FORBS } & \square \text { LOCAL FORBS } \\ \square \text { WETLAND } & \square \text { LOCAL WETLAND }\end{array}$

$\square$ WETLAND 
Hamden

Broken Arrow Nursery

13 Broken Arrow Rd

Hamden, CT

06518

$\checkmark$ LOCAL TREE

PHONE: (203) 288-1026

$\checkmark$ SHRUB $\quad \square$ LOCAL SHRUB

$\nabla$ GRASS $\quad \nabla$ LOCAL GRASS

$\square$ FORBS $\square$ LOCAL FORBS

URL: www.brokenarrownursery.com

Hartford

Connecticut Department of Environmental Protection Division of Forestry

79 Elm Street

$\square$ WETLAND $\square$ LOCAL WETLAND

Hartford, CT

06106

PHONE: (860) 424-3630

$\begin{array}{ll}\square \text { TREE } & \square \text { LOCAL TREE } \\ \square \text { SHRUB } & \square \text { LOCAL SHRUB } \\ \square \text { GRASS } & \square \text { LOCAL GRASS } \\ \square \text { FORBS } & \square \text { LOCAL FORBS } \\ \square \text { WETLAND } & \square \text { LOCAL WETLAND }\end{array}$

URL: http://dep.state ct.us/burnatr/

\section{Kensington}

Sunny Border Nurseries, Inc

1709 Kensington Rd.

Kensington, $\mathrm{CT}$

06037

$\begin{array}{ll}\square \text { TREE } & \square \text { LOCAL TREE } \\ \square \text { SHRUB } & \square \text { LOCAL SHRUB } \\ \square \text { GRASS } & \square \text { LOCAL GRASS } \\ \square \text { FORBS } & \square \text { LOCAL FORBS } \\ \square \text { WETLAND } & \square \text { LOCAL WETLAND }\end{array}$

PHONE: (860) 828-0321

$\checkmark$ WETLAND $\quad \nabla$ LOCAL WETLAND

URL: www.sunnyborder.com

Kent

Kent Horticultural Services

PO Box 128

Kent, CT

06757

PHONE: (860) 927-3480

$\begin{array}{ll}\square \text { TREE } & \square \text { LOCAL TREE } \\ \square \text { SHRUB } & \square \text { LOCAL SHRUB } \\ \square \text { GRASS } & \square \text { LOCAL GRASS } \\ \square \text { FORBS } & \square \text { LOCAL FORBS } \\ \square \text { WETLAND } & \square \text { LOCAL WETLAND }\end{array}$

URL: none currently available

Killingworth

Winterberry Nursery

104 Parker Hill Rd. Ext.

Killingworth, CT

06417

PHONE: (860) 663-2747

$\begin{array}{ll}\square \text { TREE } & \square \text { LOCAL TREE } \\ \square \text { SHRUB } & \square \text { LOCAL SHRUB } \\ \square \text { GRASS } & \square \text { LOCAL GRASS } \\ \nabla \text { FORBS } & \square \text { LOCAL FORBS } \\ \square \text { WETLAND } & \square \text { LOCAL WETLAND }\end{array}$

URL: none currently available

\section{Lebanon}

Chappell Nursery

1114 Trumbull Hwy.

Lebanon, CT

06249

PHONE: (860) 379-2626

$\begin{array}{ll}\square \text { TREE } & \square \text { LOCAL TREE } \\ \bar{\nabla} \text { SHRUB } & \square \text { LOCAL SHRUB } \\ \square \text { GRASS } & \square \text { LOCAL GRASS } \\ \square \text { FORBS } & \square \text { LOCAL FORBS } \\ \square \text { WETLAND } & \square \text { LOCAL WETLAND }\end{array}$

URL: none currently available 
Prides Corner Farm, Inc.

122 Waterman Rd

Lebanon, CT

06249

PHONE: (860) 887-2092

$\begin{array}{ll}\nabla \text { TREE } & \square \text { LOCAL TREE } \\ \nabla \text { SHRUB } & \square \text { LOCAL SHRUB } \\ \square \text { GRASS } & \square \text { LOCAL GRASS } \\ \nabla \text { FORBS } & \square \text { LOCAL FORBS } \\ \square \text { WETLAND } & \square \text { LOCAL WETLAND }\end{array}$

URL: none currently available

Ledyard

Holdridge Farm Nursery

PO Box 29

Ledyard, CT

06339

PHONE: (860) 464-8400

$\begin{array}{ll}\nabla \text { TREE } & \nabla \text { LOCAL TREE } \\ \square \text { SHRUB } & \square \text { LOCAL SHRUB } \\ \square \text { GRASS } & \square \text { LOCAL GRASS } \\ \nabla \text { FORBS } & \nabla \text { LOCAL FORBS } \\ \square \text { WETLAND } & \square \text { LOCAL WETLAND }\end{array}$

URL: none currently available

\section{Litchfield}

White Flower Farms

P.O. Box 50, Route 63

Litchfield, CT

06759

PHONE: (860) 482-3638

$\begin{array}{ll}\square \text { TREE } & \square \text { LOCAL TREE } \\ \nabla \text { SHRUB } & \boldsymbol{\nabla} \text { LOCAL SHRUB } \\ \square \text { GRASS } & \square \text { LOCAL GRASS } \\ \nabla \text { FORBS } & \boldsymbol{\nabla} \text { LOCAL FORBS } \\ \nabla \text { WETLAND } & \boldsymbol{\nabla} \text { LOCAL WETLAND }\end{array}$

URL: www.whiteflowerfarm.com

\section{Manchester}

Woodland Gardens

168 Woodland St.

Manchester, CT

$\nabla$ TREE

$\checkmark$ SHRUB

$\checkmark$ LOCAL TREE

06040

PHONE: (860) 643-8474

$\square$ GRASS

$\checkmark$ FORBS

$\checkmark$ LOCAL SHRUB

$\square$ WETLAND

$\square$ LOCAL GRASS

$\checkmark$ LOCAL FORBS

URL: none currently available

Milford

Glorias Garden Center

258 Boston Post Rd.

Milford, CT

06460

PHONE: (203) 877-2776

$\begin{array}{ll}\nabla \text { TREE } & \square \text { LOCAL TREE } \\ \square \text { SHRUB } & \square \text { LOCAL SHRUB } \\ \square \text { GRASS } & \square \text { LOCAL GRASS } \\ \square \text { FORBS } & \square \text { LOCAL FORBS } \\ \square \text { WETLAND } & \square \text { LOCAL WETLAND }\end{array}$

URL: none currently available

\section{Monroe}

Kenneth Twombly Nursery

163 Barn Hill Rd

$\checkmark$ TREE

$\checkmark$ SHRUB

$\checkmark$ LOCAL TREE

Monroe, CT

06468

PHONE: (203) 261-2133

$\square$ GRASS

$\checkmark$ FORBS

$\checkmark$ LOCAL SHRUB

$\square$ WETLAND

$\square$ LOCAL GRASS

$\checkmark$ LOCAL FORBS

$\square$ LOCAL WETLAND

URL: none currently available 


\section{New Milford}

Cortina Gardens

25 Bridgewater Rd

New Milford, CT

06776

PHONE: (860) 354-8120

URL: none currently available

\section{Fieldstone Nursery}

48 Merryall Rd.

New Milford, CT

06776

PHONE: (860) 354-3116

URL: none currently available

$\begin{array}{ll}\square \text { TREE } & \square \text { LOCAL TREE } \\ \square \text { SHRUB } & \square \text { LOCAL SHRUB } \\ \square \text { GRASS } & \square \text { LOCAL GRASS } \\ \nabla \text { FORBS } & \square \text { LOCAL FORBS } \\ \square \text { WETLAND } & \square \text { LOCAL WETLAND }\end{array}$

$\checkmark$ TREE

$\checkmark$ SHRUB

$\checkmark$ LOCAL TREE

$\checkmark$ LOCAL SHRUB

$\square$ GRASS $\square$ LOCAL GRASS

$\checkmark$ FORBS $\quad \nabla$ LOCAL FORBS

$\square$ WETLAND $\square$ LOCAL WETLAND

Newton

Planters Choice

140 Huntington Rd.

Newton, CT

06470

PHONE: (203) 426-4037

$\begin{array}{ll}\nabla \text { TREE } & \square \text { LOCAL TREE } \\ \square \text { SHRUB } & \square \text { LOCAL SHRUB } \\ \square \text { GRASS } & \square \text { LOCAL GRASS } \\ \nabla \text { FORBS } & \square \text { LOCAL FORBS } \\ \square \text { WETLAND } & \square \text { LOCAL WETLAND }\end{array}$

URL: none currently available

\section{North Haven}

Bell Nurseries, Inc.

1301 Hartford Turnpike

North Haven, CT

06473

PHONE: (203) 248-5086

$\begin{array}{ll}\nabla \text { TREE } & \square \text { LOCAL TREE } \\ \nabla \text { SHRUB } & \square \text { LOCAL SHRUB } \\ \square \text { GRASS } & \square \text { LOCAL GRASS } \\ \square \text { FORBS } & \square \text { LOCAL FORBS } \\ \square \text { WETLAND } & \square \text { LOCAL WETLAND }\end{array}$

URL: none currently available

Prospect

Prospect Nursery \& Garden

246 New Haven Rd.

Prospect, CT

06712

PHONE: (203) 758-4909

$\begin{array}{ll}\square \text { TREE } & \square \text { LOCAL TREE } \\ \square \text { SHRUB } & \square \text { LOCAL SHRUB } \\ \square \text { GRASS } & \square \text { LOCAL GRASS } \\ \square \text { FORBS } & \square \text { LOCAL FORBS } \\ \square \text { WETLAND } & \square \text { LOCAL WETLAND }\end{array}$

URL: none currently available

\section{Rocky Hill}

A.J. Vicino \& Sons Nursery

259 New Britain Ave.

Rocky Hill, CT

06067

PHONE: (860) 529-1304

$\begin{array}{ll}\nabla \text { TREE } & \square \text { LOCAL TREE } \\ \nabla \text { SHRUB } & \square \text { LOCAL SHRUB } \\ \square \text { GRASS } & \square \text { LOCAL GRASS } \\ \square \text { FORBS } & \square \text { LOCAL FORBS } \\ \square \text { WETLAND } & \square \text { LOCAL WETLAND }\end{array}$

URL: none currently available 
Salem

Salem Country Gardens

385 New London Rd.

Salem, CT

06415

PHONE: (860) 435-2439

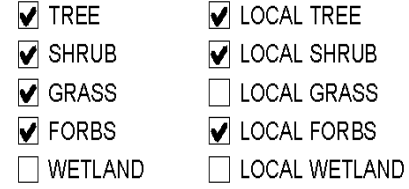

URL: none currently available

Sharon

Beardsley Gardens

157 Gay St., Rt. 41

Sharon, CT

06069

PHONE: (860) 364-0727

$\begin{array}{ll}\nabla \text { TREE } & \square \text { LOCAL TREE } \\ \nabla \text { SHRUB } & \square \text { LOCAL SHRUB } \\ \square \text { GRASS } & \square \text { LOCAL GRASS } \\ \nabla \text { FORBS } & \square \text { LOCAL FORBS } \\ \nabla \text { WETLAND } & \square \text { LOCAL WETLAND }\end{array}$

URL: www.beardsleygardens.com

\section{Simsbury}

Warners Nursery Center

PO Box 662

Simsbury, CT

06070

PHONE: (860) 651-0204

$\begin{array}{ll}\nabla \text { TREE } & \square \text { LOCAL TREE } \\ \square \text { SHRUB } & \square \text { LOCAL SHRUB } \\ \square \text { GRASS } & \square \text { LOCAL GRASS } \\ \nabla \text { FORBS } & \square \text { LOCAL FORBS } \\ \square \text { WETLAND } & \square \text { LOCAL WETLAND }\end{array}$

URL: none currently available

\section{Southington}

Evergreen Nursery, Inc.

567 Woodruff St.

Southington, CT

06489

PHONE: (860) 628-0325

$\begin{array}{ll}\square \text { TREE } & \square \text { LOCAL TREE } \\ \nabla \text { SHRUB } & \square \text { LOCAL SHRUB } \\ \square \text { GRASS } & \square \text { LOCAL GRASS } \\ \square \text { FORBS } & \square \text { LOCAL FORBS } \\ \square \text { WETLAND } & \square \text { LOCAL WETLAND }\end{array}$

URL: none currently available

\section{Stamford}

High Ridge Nursery

1854 High Ridge Rd.

Stamford, CT

06903

PHONE: (203) 329-9957

$\begin{array}{ll}\nabla \text { TREE } & \nabla \text { LOCAL TREE } \\ \nabla \text { SHRUB } & \square \text { LOCAL SHRUB } \\ \square \text { GRASS } & \square \text { LOCAL GRASS } \\ \nabla \text { FORBS } & \square \text { LOCAL FORBS } \\ \square \text { WETLAND } & \square \text { LOCAL WETLAND }\end{array}$

URL: none currently available

\section{Watertown}

James S. Hosking Nursery

PO Box 6

Watertown, CT

06795

PHONE: (860) 274-8889

$\begin{array}{ll}\nabla \text { TREE } & \square \text { LOCAL TREE } \\ \square \text { SHRUB } & \square \text { LOCAL SHRUB } \\ \square \text { GRASS } & \square \text { LOCAL GRASS } \\ \square \text { FORBS } & \square \text { LOCAL FORBS } \\ \square \text { WETLAND } & \square \text { LOCAL WETLAND }\end{array}$

URL: none currently available 


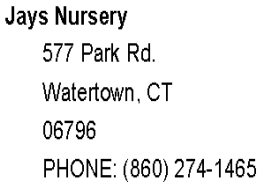

URL: none currently available

\section{West Suffield}

Robert Baker, Inc.

1700 Mountain Rd.

West Suffield, CT

06093

PHONE: (860) 668-7371

URL: none currently available

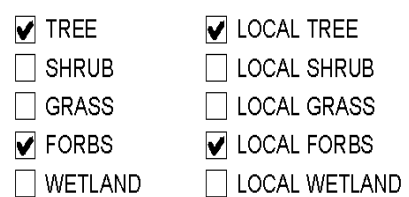

$\square$ WETLAND

\section{n}

Youngs Nurseries, Inc.

211 Danbury Rd.

Wilton, CT

0689 ?

PHONE: (203) 762-5511

$\begin{array}{ll}\square \text { TREE } & \square \text { LOCAL TREE } \\ \square \text { SHRUB } & \square \text { LOCAL SHRUB } \\ \square \text { GRASS } & \square \text { LOCAL GRASS } \\ \square \text { FORBS } & \square \text { LOCAL FORBS } \\ \square \text { WETLAND } & \square \text { LOCAL WETLAND }\end{array}$

$\square$ WETLAND $\square$ LOCAL WETLAND

URL: none currently available

Windsor Locks

Arthur Boglisch \& Sons

11 Roberts $S t$

Windsor Locks, CT

06096

PHONE: (860) 623-1704

$\begin{array}{ll}\nabla \text { TREE } & \square \text { LOCAL TREE } \\ \nabla \text { SHRUB } & \square \text { LOCAL SHRUB } \\ \square \text { GRASS } & \square \text { LOCAL GRASS } \\ \nabla \text { FORBS } & \square \text { LOCAL FORBS } \\ \square \text { WETLAND } & \square \text { LOCAL WETLAND }\end{array}$

$\square$ WETLAND $\square$ LOCAL WETLAND

URL: none currently available

Yantic

River Run Nursery

PO Box 155

Yantic, CT

06389

PHONE: (860) 887-2092

$\begin{array}{ll}\square \text { TREE } & \square \text { LOCAL TREE } \\ \square \text { SHRUB } & \square \text { LOCAL SHRUB } \\ \square \text { GRASS } & \square \text { LOCAL GRASS } \\ \square \text { FORBS } & \square \text { LOCAL FORBS } \\ \square \text { WETLAND } & \square \text { LOCAL WETLAND }\end{array}$

URL: none currently available

\section{Delaware}

Claymont

Sweeney Seed Company, Inc.

2721 Philadelphia Pike

Claymont, DE

19703

PHONE: (302) 798-6001

$\begin{array}{ll}\square \text { TREE } & \square \text { LOCAL TREE } \\ \square \text { SHRUB } & \square \text { LOCAL SHRUB } \\ \square \text { GRASS } & \square \text { LOCAL GRASS } \\ \square \text { FORBS } & \square \text { LOCAL FORBS } \\ \square \text { WETLAND } & \square \text { LOCAL WETLAND }\end{array}$

$\square$ LOCAL WETLAND

URL: www.Sweeneyseed.com 
Clayton

Forest View Nursery, Inc.

1313 Blackbird Forest Rd

Clayton, DE

19938

TREE

$\checkmark$ SHRUB

$\checkmark$ GRASS

$\checkmark$ LOCAL TREE

PHONE: (302) 653-7757

$\square$ FORBS

$\nabla$ WETLAND $\nabla$ LOCAL WETLAND

URL: www.forestviewnursery.com

Hartly

Shelterwood Farm

179 Tuxward Road

Hartly, DE

19953

PHONE: (302) 492-8071

$\begin{array}{ll}\nabla \text { TREE } & \square \text { LOCAL TREE } \\ \square \text { SHRUB } & \square \text { LOCAL SHRUB } \\ \square \text { GRASS } & \square \text { LOCAL GRASS } \\ \square \text { FORBS } & \square \text { LOCAL FORBS } \\ \square \text { WETLAND } & \square \text { LOCAL WETLAND }\end{array}$

URL: none currently available

Lewes

Cape Farms Acres

RD1, Box E134A"

Lewes, DE

19958

PHONE: (302) 945-1840

$\begin{array}{ll}\square \text { TREE } & \square \text { LOCAL TREE } \\ \square \text { SHRUB } & \square \text { LOCAL SHRUB } \\ \square \text { GRASS } & \square \text { LOCAL GRASS } \\ \square \text { FORBS } & \square \text { LOCAL FORBS } \\ \square \text { WETLAND } & \square \text { LOCAL WETLAND }\end{array}$

URL: none currently available

Newark

Sweeney Seed Company, Inc.

Suite 950, Peoples Plaza"

Newark, DE

19702

$\begin{array}{ll}\square \text { TREE } & \square \text { LOCAL TREE } \\ \square \text { SHRUB } & \square \text { LOCAL SHRUB } \\ \square \text { GRASS } & \square \text { LOCAL GRASS } \\ \square \text { FORBS } & \square \text { LOCAL FORBS } \\ \square \text { WETLAND } & \square \text { LOCAL WETLAND }\end{array}$

PHONE: (302) 834-0440

$\square$ WETLAND $\square$ LOCAL WETLAND

URL: wWw.Sweeneyseed.com

Smyrna

Joseph Wick Nurseries

3902 Branford Rd.

Smyrna, DE

19977

PHONE: (302) 653-9000

$\begin{array}{ll}\nabla \text { TREE } & \nabla \text { LOCAL TREE } \\ \nabla \text { SHRUB } & \square \text { LOCAL SHRUB } \\ \nabla \text { GRASS } & \square \text { LOCAL GRASS } \\ \square \text { FORBS } & \square \text { LOCAL FORBS } \\ \nabla \text { WETLAND } & \nabla \text { LOCAL WETLAND }\end{array}$

URL: none currently available

\section{Florida}

Alachua

Alachua Farm \& Lumber Center

Box 2290

Alachua, FL

32616

PHONE: (386) 462-3003

$\begin{array}{ll}\square \text { TREE } & \square \text { LOCAL TREE } \\ \square \text { SHRUB } & \square \text { LOCAL SHRUB } \\ \square \text { GRASS } & \square \text { LOCAL GRASS } \\ \square \text { FORBS } & \square \text { LOCAL FORBS } \\ \square \text { WETLAND } & \square \text { LOCAL WETLAND }\end{array}$

URL: none currently available 
Possum Hollow Farms

10106 NW 156th Ave.

Alachua, FL

32615

PHONE: (905) 462-5455

$\begin{array}{ll}\square \text { TREE } & \square \text { LOCAL TREE } \\ \square \text { SHRUB } & \square \text { LOCAL SHRUB } \\ \square \text { GRASS } & \square \text { LOCAL GRASS } \\ \square \text { FORBS } & \square \text { LOCAL FORBS } \\ \square \text { WETLAND } & \square \text { LOCAL WETLAND }\end{array}$

URL: none currently available

Arcadia

Deluxe Trees \& Shrubs

6306 SW Carlton Ave

Arcadia, FL

$\nabla$ TREE $\quad \nabla$ LOCAL TREE

34266

PHONE: (863) 494-1488

$\nabla$ SHRUB $\nabla$ LOCAL SHRUB

$\square$ GRASS $\square$ LOCAL GRASS

$\square$ FORBS $\square$ LOCAL FORBS

$\square$ WETLAND $\square$ LOCAL WETLAND

URL: www.afnn.org

\section{Avon Park}

Normans Native Plants Plus

2150 US $27 \mathrm{~N}$

Avon Park, FL

33825

PHONE: (863) 414-4729

$\begin{array}{ll}\nabla \text { TREE } & \square \text { LOCAL TREE } \\ \square \text { SHRUB } & \square \text { LOCAL SHRUB } \\ \square \text { GRASS } & \square \text { LOCAL GRASS } \\ \square \text { FORBS } & \square \text { LOCAL FORBS } \\ \nabla \text { WETLAND } & \square \text { LOCAL WETLAND }\end{array}$

URL: www.afnn.org

Bartow

Bartow Ornamental Nursery

3890 Hwy $60 \mathrm{E}$

Bartow, FL

33830

PHONE: (888) 534-1350

$\begin{array}{ll}\nabla \text { TREE } & \square \text { LOCAL TREE } \\ \square \text { SHRUB } & \square \text { LOCAL SHRUB } \\ \square \text { GRASS } & \square \text { LOCAL GRASS } \\ \square \text { FORBS } & \square \text { LOCAL FORBS } \\ \square \text { WETLAND } & \square \text { LOCAL WETLAND }\end{array}$

URL: http://Www.southeasttrees.com

Bookelea

Soaring Eagle Nursery

5101 Pine Island Rd

Bookeleâ, FL

33922

PHONE: (800) 293-7256

$\begin{array}{ll}\nabla \text { TREE } & \square \text { LOCAL TREE } \\ \nabla \text { SHRUB } & \square \text { LOCAL SHRUB } \\ \square \text { GRASS } & \square \text { LOCAL GRASS } \\ \square \text { FORBS } & \square \text { LOCAL FORBS } \\ \square \text { WETLAND } & \square \text { LOCAL WETLAND }\end{array}$

URL: none currently available

\section{Bookelia}

Pine Breeze Nursery

PO Box 0702

Bookelia, FL

33922-070

PHONE: (941) 283-7200

$\begin{array}{ll}\nabla \text { TREE } & \square \text { LOCAL TREE } \\ \square \text { SHRUB } & \square \text { LOCAL SHRUB } \\ \nabla \text { GRASS } & \square \text { LOCAL GRASS } \\ \nabla \text { FORBS } & \nabla \text { LOCAL FORBS } \\ \square \text { WETLAND } & \square \text { LOCAL WETLAND }\end{array}$

URL: www.afnn.org 
Boynton Beach

Boynton Botanicals

9281 87th PLS

Boynton Beach, FL

33437

PHONE: (561) 737-1490

URL: http://Www.afnn.org

Native Green Cay

12750 Hagen Ranch Rd

Boynton Beach, FL

33437

PHONE: (561) 496-1415

URL: www.afnn.org

Pat Fords Nursery, Inc. 840096 th CT S

Boynton Beach, FL

33437

PHONE: (561) 734-7188

$\begin{array}{ll}\nabla \text { TREE } & \square \text { LOCAL TREE } \\ \square \text { SHRUB } & \square \text { LOCAL SHRUB } \\ \nabla \text { GRASS } & \square \text { LOCAL GRASS } \\ \nabla \text { FORBS } & \square \text { LOCAL FORBS } \\ \nabla \text { WETLAND } & \square \text { LOCAL WETLAND }\end{array}$

$\checkmark$ TREE

$\square$ SHRUB

$\square$ GRASS

$\square$ FORBS

$\square$ WETLAND

$\checkmark$ LOCAL TREE

$\square$ LOCAL SHRUB

$\square$ LOCAL GRASS

$\square$ LOCAL FORBS

$\square$ LOCAL WETLAND

$\begin{array}{ll}\square \text { TREE } & \nabla \text { LOCAL TREE } \\ \square \text { SHRUB } & \square \text { LOCAL SHRUB } \\ \nabla \text { GRASS } & \nabla \text { LOCAL GRASS } \\ \square \text { FORBS } & \square \text { LOCAL FORBS } \\ \square \text { WETLAND } & \square \text { LOCAL WETLAND }\end{array}$

URL: www.afnn.org

Bradenton

Sabay Tree Farm \& Nursery, Inc.

POB 1686

Bradenton, $\mathrm{FL}$

34206

PHONE: (941) 322-2367

$\begin{array}{ll}\nabla \text { TREE } & \square \text { LOCAL TREE } \\ \nabla \text { SHRUB } & \nabla \text { LOCAL SHRUB } \\ \square \text { GRASS } & \square \text { LOCAL GRASS } \\ \square \text { FORBS } & \square \text { LOCAL FORBS } \\ \square \text { WETLAND } & \square \text { LOCAL WETLAND }\end{array}$

URL: www.afnn.org

\section{Brooker}

Wild Azalea Nursery

17970 SW 111th St

Brooker, FL

32622

PHONE: (352) 485-3556

$\begin{array}{ll}\nabla \text { TREE } & \square \text { LOCAL TREE } \\ \nabla \text { SHRUB } & \square \text { LOCAL SHRUB } \\ \square \text { GRASS } & \square \text { LOCAL GRASS } \\ \square \text { FORBS } & \square \text { LOCAL FORBS } \\ \square \text { WETLAND } & \square \text { LOCAL WETLAND }\end{array}$

URL: none currently available

\section{Brooksville}

Hickory Hill Native Nursery, Inc.

27212 Hickory Rd.

Brooksville, FL

34602

PHONE: (352) 754-9701

$\begin{array}{ll}\nabla \text { TREE } & \nabla \text { LOCAL TREE } \\ \nabla \text { SHRUB } & \nabla \text { LOCAL SHRUB } \\ \nabla \text { GRASS } & \nabla \text { LOCAL GRASS } \\ \nabla \text { FORBS } & \nabla \text { LOCAL FORBS } \\ \nabla \text { WETLAND } & \nabla \text { LOCAL WETLAND }\end{array}$

URL: www.afnn.org 
Plant Materials Center

14119 Broad St

Brooksville, FL

34601

PHONE: (352) 796-9600

$\begin{array}{ll}\square \text { TREE } & \square \text { LOCAL TREE } \\ \square \text { SHRUB } & \square \text { LOCAL SHRUB } \\ \square \text { GRASS } & \square \text { LOCAL GRASS } \\ \square \text { FORBS } & \square \text { LOCAL FORBS } \\ \square \text { WETLAND } & \square \text { LOCAL WETLAND }\end{array}$

URL: http://plant-materials.nrcs.usda.gov/flpmc/

\section{Chiefland}

A.D. Andrews Nursery

$\begin{array}{lll}\text { Box } 1126 & \square \text { TREE } & \square \text { LOCAL TREE } \\ \text { Chiefland, FL } & \square \text { SHRUB } & \square \text { LOCAL SHRUB } \\ 32626 & \square \text { GRASS } & \square \text { LOCAL GRASS } \\ \text { PHONE: (352) 493-2496 } & \square \text { FORBS } & \square \text { LOCAL FORBS } \\ & \square \text { WETLAND } & \square \text { LOCAL WETLAND }\end{array}$

URL: none currently available

\section{Christmas}

Green Images

P.O. Box 1330

Christmas, FL

32701

PHONE: (407) 568-1333

$\begin{array}{ll}\nabla \text { TREE } & \nabla \text { LOCAL TREE } \\ \nabla \text { SHRUB } & \nabla \text { LOCAL SHRUB } \\ \nabla \text { GRASS } & \nabla \text { LOCAL GRASS } \\ \nabla \text { FORBS } & \boldsymbol{\nabla} \text { LOCAL FORBS } \\ \nabla \text { WETLAND } & \boldsymbol{\nabla} \text { LOCAL WETLAND }\end{array}$

URL: http://Www.afinn.org

\section{Clearwater}

Whisper Palms of Terra Ceia LLC

4400118 th Ave N STE 305

Clearwater, FL

33762

PHONE: (727) 573-1292

$\begin{array}{ll}\square \text { TREE } & \square \text { LOCAL TREE } \\ \square \text { SHRUB } & \square \text { LOCAL SHRUB } \\ \square \text { GRASS } & \nabla \text { LOCAL GRASS } \\ \nabla \text { FORBS } & \square \text { LOCAL FORBS } \\ \square \text { WETLAND } & \square \text { LOCAL WETLAND }\end{array}$

URL: www.afnn.org

\section{Croveland}

Dixie Plant Farm

6037 Lake Erie Rd

Croveland. FL

34736

PHONE: (352) 429-5380

$\begin{array}{ll}\nabla \text { TREE } & \square \text { LOCAL TREE } \\ \nabla \text { SHRUB } & \square \text { LOCAL SHRUB } \\ \nabla \text { GRASS } & \nabla \text { LOCAL GRASS } \\ \square \text { FORBS } & \square \text { LOCAL FORBS } \\ \nabla \text { WETLAND } & \checkmark \text { LOCAL WETLAND }\end{array}$

URL: none currently available

\section{Davenport}

\section{The Natives}

2929 J.B. Carter Rd.

Davenport, $F L$

33837

PHONE: (863) 422-6664

$\begin{array}{ll}\nabla \text { TREE } & \nabla \text { LOCAL TREE } \\ \nabla \text { SHRUB } & \checkmark \text { LOCAL SHRUB } \\ \nabla \text { GRASS } & \nabla \text { LOCAL GRASS } \\ \nabla \text { FORBS } & \nabla \text { LOCAL FORBS } \\ \nabla \text { WETLAND } & \nabla \text { LOCAL WETLAND }\end{array}$

URL: http://Ww'w afinn.org 
Davie

Alexander Landscaping \& Plant Farm

910 S Flamingo Rd

Davie, FL

33325-440

PHONE: (954) 472-5039

URL: http:/Wwww.alexfarm.com

Garden of Delights

14560 SW 14th St

Davie, FL

33325

PHONE: (954) 370-9004

$\checkmark$ TREE $\checkmark$ LOCAL TREE

$\nabla$ SHRUB $\quad \nabla$ LOCAL SHRUB

$\checkmark$ GRASS $\square$ LOCAL GRASS

$\nabla$ FORBS $\quad \nabla$ LOCAL FORBS

$\nabla$ WETLAND $\nabla$ LOCAL WETLAND

URL: www.gardenofdelights.com

Johns Patio Stone \& Sod Inc.

$1130 \mathrm{~S}$. Flamingo Rd

$\checkmark$ TREE $\quad \nabla$ LOCAL TREE

Davie, FL

33325

$\checkmark$ SHRUB

$\checkmark$ LOCAL SHRUB

PHONE: (954) 475-1558

$\nabla$ GRASS $\quad \nabla$ LOCAL GRASS

$\square$ FORBS $\square$ LOCAL FORBS

$\square$ WETLAND $\square$ LOCAL WETLAND

URL: none currently available

\section{Delray Beach}

Tropical Plant \& Seed Locators

Box 1000

Delray Beach, FL

33447

$\boldsymbol{v}$ TREE

$\boldsymbol{\nabla}$ LOCAL TREE

PHONE: (561) 278-6004

SHRUB $\square$ LOCAL SHRUB

$\square$ GRASS $\square$ LOCAL GRASS

$\square$ FORBS $\square$ LOCAL FORBS

$\square$ WETLAND $\square$ LOCAL WETLAND

URL: none currently available

\section{Fort Myers}

All Native Garden \& Plant Nursery

300 Center Road

Fort Myers, FL

33907-151

PHONE: (239) 939-9663

$\begin{array}{ll}\nabla \text { TREE } & \nabla \text { LOCAL TREE } \\ \nabla \text { SHRUB } & \nabla \text { LOCAL SHRUB } \\ \nabla \text { GRASS } & \nabla \text { LOCAL GRASS } \\ \nabla \text { FORBS } & \square \text { LOCAL FORBS } \\ \nabla \text { WETLAND } & \nabla \text { LOCAL WETLAND }\end{array}$

URL: http://nolawn.com

Rigsby Nursery, Inc.

PO Box 50910

Fort Myers, FL

33994-091

PHONE: (239) 543-3379

$\begin{array}{ll}\nabla \text { TREE } & \boldsymbol{\nabla} \text { LOCAL TREE } \\ \square \text { SHRUB } & \square \text { LOCAL SHRUB } \\ \square \text { GRASS } & \square \text { LOCAL GRASS } \\ \square \text { FORBS } & \square \text { LOCAL FORBS } \\ \square \text { WETLAND } & \square \text { LOCAL WETLAND }\end{array}$

URL: www.cmione.com 
Fort Pierce

Diamond R Fertilizer

4100 Glades Cut-Off Road

Fort Pierce, FL

34981

PHONE: (772) 464-9300

$\begin{array}{ll}\square \text { TREE } & \square \text { LOCAL TREE } \\ \square \text { SHRUB } & \square \text { LOCAL SHRUB } \\ \square \text { GRASS } & \square \text { LOCAL GRASS } \\ \square \text { FORBS } & \square \text { LOCAL FORBS } \\ \square \text { WETLAND } & \square \text { LOCAL WETLAND }\end{array}$

URL: http://Ww'w. diamond-r.com

\section{Ft. Lauderdale}

Runway Growers, Inc.

2891 S.W. 36th St

Ft. Lauderdale, FL

33312

PHONE: (954) 580-0269

$\begin{array}{ll}\nabla \text { TREE } & \nabla \text { LOCAL TREE } \\ \nabla \text { SHRUB } & \checkmark \text { LOCAL SHRUB } \\ \nabla \text { GRASS } & \square \text { LOCAL GRASS } \\ \nabla \text { FORBS } & \checkmark \text { LOCAL FORBS } \\ \square \text { WETLAND } & \square \text { LOCAL WETLAND }\end{array}$

URL: none currently available

\section{Ft. Meade}

Reclamation Trees

1857 Keller Rd

Ft. Meade, FL

33841

PHONE: (941) 285-8130

$\begin{array}{ll}\nabla \text { TREE } & \square \text { LOCAL TREE } \\ \square \text { SHRUB } & \square \text { LOCAL SHRUB } \\ \square \text { GRASS } & \square \text { LOCAL GRASS } \\ \square \text { FORBS } & \square \text { LOCAL FORBS } \\ \square \text { WETLAND } & \square \text { LOCAL WETLAND }\end{array}$

URL: none currently available

\section{Gainesville}

San Felasco Nurseries, Inc

$7315 \mathrm{NW} 126 \mathrm{St}$

$\checkmark$ TREE $\quad \nabla$ LOCAL TREE

Gainesville, FL

32653

PHONE: (800) 933-9638

$\checkmark$ SHRUB

$\square$ GRASS

$\checkmark$ FORBS

$\checkmark$ LOCAL SHRUB

$\checkmark$ WETLAND

$\checkmark$ LOCAL GRASS

$\nabla$ LOCAL FORBS

URL: www.sanfelasco.com

Tropic Traditions Nurseries

PO Box 13678

Gainesville, FL

32604-167

PHONE: (888) 586-5875

$\begin{array}{ll}\nabla \text { TREE } & \square \text { LOCAL TREE } \\ \square \text { SHRUB } & \square \text { LOCAL SHRUB } \\ \square \text { GRASS } & \square \text { LOCAL GRASS } \\ \square \text { FORBS } & \square \text { LOCAL FORBS } \\ \square \text { WETLAND } & \square \text { LOCAL WETLAND }\end{array}$

URL: www.tropictraditions.com

\section{Goulds}

Native Tree Nursery, Inc.

17250 S.W. 232nd St.

Goulds, FL

33170

PHONE: (305) 247-4499

$\begin{array}{ll}\nabla \text { TREE } & \checkmark \text { LOCAL TREE } \\ \nabla \text { SHRUB } & \checkmark \text { LOCAL SHRUB } \\ \nabla \text { GRASS } & \checkmark \text { LOCAL GRASS } \\ \square \text { FORBS } & \square \text { LOCAL FORBS } \\ \square \text { WETLAND } & \checkmark \text { LOCAL WETLAND }\end{array}$

URL: hlttp:/WwW nativetreenursery.com 
Groveland

Green Isle Gardens

6043 Lake Erie Rd.

Groveland, $\mathrm{FL}$

34736

PHONE: (321) 436-4932

$\begin{array}{ll}\square \text { TREE } & \square \text { LOCAL TREE } \\ \square \text { SHRUB } & \square \text { LOCAL SHRUB } \\ \square \text { GRASS } & \square \text { LOCAL GRASS } \\ \square \text { FORBS } & \square \text { LOCAL FORBS } \\ \square \text { WETLAND } & \square \text { LOCAL WETLAND }\end{array}$

URL: www.afnn.org

\section{Gulf Breeze}

The Garden Gate

3268 Fordham Parkway

Gulf Breeze, FL

32561

PHONE: (850) 932-9066

$\begin{array}{ll}\square \text { TREE } & \square \text { LOCAL TREE } \\ \square \text { SHRUB } & \square \text { LOCAL SHRUB } \\ \square \text { GRASS } & \square \text { LOCAL GRASS } \\ \square \text { FORBS } & \square \text { LOCAL FORBS } \\ \square \text { WETLAND } & \square \text { LOCAL WETLAND }\end{array}$

URL: www.afnn.org

Havana

Florida Wildflower Cooperative

$\begin{array}{lll}\text { PO Box } 1228 & \square \text { TREE } & \square \text { LOCAL TREE } \\ \text { Havana, FL } & \square \text { SHRUB } & \square \text { LOCAL SHRUB } \\ 32333 & \nabla \text { GRASS } & \nabla \text { LOCAL GRASS } \\ \text { PHONE: (850) 539-2999 } & \nabla \text { FORBS } & \nabla \text { LOCAL FORBS } \\ & \nabla \text { WETLAND } & \nabla \text { LOCAL WETLAND }\end{array}$

URL: wnw.floridawildflowers.com

\section{Hawthorn}

Breezy Oaks Nursery

23602 S.E. Hawthorn Rd.

$\boldsymbol{\nabla}$ TREE $\quad \boldsymbol{\nabla}$ LOCAL TREE

Hawthorn, FL

32640

PHONE: (352) 481-3795

$\nabla$ SHRUB $\quad \nabla$ LOCAL SHRUB

$\square$ GRASS $\square$ LOCAL GRASS

$\square$ FORBS $\square$ LOCAL FORBS

$\square$ WETLAND $\square$ LOCAL WETLAND

URL: http:/Nwww.afnn.org

\section{Homestead}

Arvida Nurseries

P.O. Box 1508

Homestead, FL

33090-150

PHONE: (305) 245-9573

$\begin{array}{ll}\nabla \text { TREE } & \square \text { LOCAL TREE } \\ \nabla \text { SHRUB } & \square \text { LOCAL SHRUB } \\ \square \text { GRASS } & \square \text { LOCAL GRASS } \\ \square \text { FORBS } & \square \text { LOCAL FORBS } \\ \square \text { WETLAND } & \square \text { LOCAL WETLAND }\end{array}$

URL: http://afnn.org

Botanics Wholesale

31701 SW 194th Ave

Homestead, FL

33031

PHONE: (305) 245-2966

$\begin{array}{ll}\square \text { TREE } & \square \text { LOCAL TREE } \\ \square \text { SHRUB } & \square \text { LOCAL SHRUB } \\ \square \text { GRASS } & \square \text { LOCAL GRASS } \\ \square \text { FORBS } & \square \text { LOCAL FORBS } \\ \square \text { WETLAND } & \square \text { LOCAL WETLAND }\end{array}$

URL: www.botanics.com 
Plant Creations Inc.

28301 SW 172 Ave

Homestead, FL

33030

PHONE: (305) 248-8147

$\begin{array}{ll}\nabla \text { TREE } & \nabla \text { LOCAL TREE } \\ \nabla \text { SHRUB } & \nabla \text { LOCAL SHRUB } \\ \nabla \text { GRASS } & \nabla \text { LOCAL GRASS } \\ \nabla \text { FORBS } & \nabla \text { LOCAL FORBS } \\ \nabla \text { WETLAND } & \nabla \text { LOCAL WETLAND }\end{array}$

URL: Www.plantcreations.com

The Liner Distributor, Inc.

18875 Southwest 272nd Street

Homestead, FL

33031

PHONE: (305) 247-5568

$\begin{array}{ll}\square \text { TREE } & \square \text { LOCAL TREE } \\ \nabla \text { SHRUB } & \square \text { LOCAL SHRUB } \\ \nabla \text { GRASS } & \square \text { LOCAL GRASS } \\ \square \text { FORBS } & \square \text { LOCAL FORBS } \\ \square \text { WETLAND } & \square \text { LOCAL WETLAND }\end{array}$

URL: none currently available

Vebers Jungle Garden

24605 SW 197 th Ave

Homestead, FL

33031

PHONE: (305) 242-9500

$\begin{array}{ll}\nabla \text { TREE } & \square \text { LOCAL TREE } \\ \nabla \text { SHRUB } & \boldsymbol{\nabla} \text { LOCAL SHRUB } \\ \nabla \text { GRASS } & \boldsymbol{\nabla} \text { LOCAL GRASS } \\ \nabla \text { FORBS } & \boldsymbol{\nabla} \text { LOCAL FORBS } \\ \square \text { WETLAND } & \square \text { LOCAL WETLAND }\end{array}$

URL: www.afnn.org

Homosassa

Nicks Nursery

PO Box 2761

Homosassa, FL

34447

PHONE: (352) 628-3098

$\begin{array}{ll}\nabla \text { TREE } & \square \text { LOCAL TREE } \\ \nabla \text { SHRUB } & \square \text { LOCAL SHRUB } \\ \square \text { GRASS } & \square \text { LOCAL GRASS } \\ \square \text { FORBS } & \square \text { LOCAL FORBS } \\ \square \text { WETLAND } & \square \text { LOCAL WETLAND }\end{array}$

URL: none currently available

Hudson

Environmental Equities, Inc.

P.O. Box 7180

$\nabla$ TREE $\quad \nabla$ LOCAL TREE

Hudson, FL

$\checkmark$ SHRUB

$\nabla$ GRASS

$\checkmark$ LOCAL SHRUB

34674-718

$\nabla$ FORBS

$\checkmark$ LOCAL GRASS

PHONE: (941) 355-1267

$\checkmark$ WETLAND

$\nabla$ LOCAL FORBS

$\checkmark$ LOCAL WETLAND

URL: www.afnn.org

Jacksonville
Mandarin Native Plants
13500 Mandarin Rd
Jacksonville, FL
32233
PHONE: (904) 268-2904
URL: none currently available
Native and Uncommon Plants
4157 Ortega Blvd
Jacksonville, FL
32210-442
PHONE: (904) $388-9851$

URL: www.nativeanduncommonplants.com

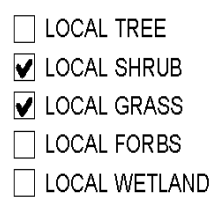

$\square$ WETLAND $\square$ LOCAL WETLAND

$\begin{array}{ll}\nabla \text { TREE } & \square \text { LOCAL TREE } \\ \nabla \text { SHRUB } & \nabla \text { LOCAL SHRUB } \\ \nabla \text { GRASS } & \checkmark \text { LOCAL GRASS } \\ \nabla \text { FORBS } & \nabla \text { LOCAL FORBS } \\ \square \text { WETLAND } & \square \text { LOCAL WETLAND }\end{array}$


Okeefenokee Growers

Box 4488

Jacksonville, FL

32201

PHONE: (904) 356-4881

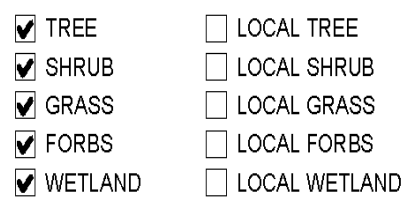

URL: none currently available

Jupiter

Rood Landscape Inc.

4546 County Line Rd

Jupiter, FL

33469

PHONE: (561) 746-5186

$\begin{array}{ll}\nabla \text { TREE } & \square \text { LOCAL TREE } \\ \nabla \text { SHRUB } & \square \text { LOCAL SHRUB } \\ \square \text { GRASS } & \square \text { LOCAL GRASS } \\ \square \text { FORBS } & \square \text { LOCAL FORBS } \\ \square \text { WETLAND } & \square \text { LOCAL WETLAND }\end{array}$

URL: www.roodlandscape.com

\section{Keystone Heights}

Echo Nursery

6615 County Road 214

Keystone Heights, FL

32656

PHONE: (800) 915-7467

$\begin{array}{ll}\square \text { TREE } & \square \text { LOCAL TREE } \\ \square \text { SHRUB } & \square \text { LOCAL SHRUB } \\ \square \text { GRASS } & \square \text { LOCAL GRASS } \\ \square \text { FORBS } & \square \text { LOCAL FORBS } \\ \square \text { WETLAND } & \square \text { LOCAL WETLAND }\end{array}$

URL: wWw.afnn.org

\section{Trail Ridge Nursery}

PO Box 967

Keystone Heights, FL

32656

PHONE: (352) 473-2073

$\begin{array}{ll}\nabla \text { TREE } & \square \text { LOCAL TREE } \\ \square \text { SHRUB } & \square \text { LOCAL SHRUB } \\ \square \text { GRASS } & \square \text { LOCAL GRASS } \\ \square \text { FORBS } & \square \text { LOCAL FORBS } \\ \square \text { WETLAND } & \square \text { LOCAL WETLAND }\end{array}$

URL: www.afnn.org

\section{Kissimmee}

Marcal Growers

1850 Palmetto Dr.

Kissimmee, FL

34744

PHONE: (407) 348-0304

$\begin{array}{ll}\nabla \text { TREE } & \nabla \text { LOCAL TREE } \\ \nabla \text { SHRUB } & \nabla \text { LOCAL SHRUB } \\ \nabla \text { GRASS } & \nabla \text { LOCAL GRASS } \\ \square \text { FORBS } & \square \text { LOCAL FORBS } \\ \nabla \text { WETLAND } & \nabla \text { LOCAL WETLAND }\end{array}$

URL: none currently available

\section{LaBelle}

Perkins Nursery, Inc.

PO Box 2460

LaBelle, FL

33975

PHONE: (865) 675-3006

$\begin{array}{ll}\nabla \text { TREE } & \square \text { LOCAL TREE } \\ \square \text { SHRUB } & \square \text { LOCAL SHRUB } \\ \square \text { GRASS } & \square \text { LOCAL GRASS } \\ \square \text { FORBS } & \square \text { LOCAL FORBS } \\ \square \text { WETLAND } & \square \text { LOCAL WETLAND }\end{array}$

URL: www.afnn.org 


\section{Lake Worth}

Erhardt Nursery

5099 2nd Road

Lake Worth, FL

33467

PHONE: (561) 967-7181

$\begin{array}{ll}\nabla \text { TREE } & \square \text { LOCAL TREE } \\ \square \text { SHRUB } & \square \text { LOCAL SHRUB } \\ \nabla \text { GRASS } & \square \text { LOCAL GRASS } \\ \nabla \text { FORBS } & \square \text { LOCAL FORBS } \\ \square \text { WETLAND } & \square \text { LOCAL WETLAND }\end{array}$

URL: www.afnn.org

Farnsworth Farms Nursery

7080 Hypoluxo Farms Rd

Lake Worth, FL

33463

PHONE: (561) 965-2657

URL: none currently available

Indian Trails Native Nursery

6315 Park LN W

$\checkmark$ TREE $\quad \nabla$ LOCAL TREE

$\nabla$ SHRUB $\square$ LOCAL SHRUB

$\square$ GRASS $\square$ LOCAL GRASS

$\checkmark$ FORBS $\quad \square$ LOCAL FORBS

$\square$ WETLAND $\square$ LOCAL WETLAND

Lake Worth, FL

33467-660

PHONE: (561) 641-9488

$\begin{array}{ll}\nabla \text { TREE } & \nabla \text { LOCAL TREE } \\ \nabla \text { SHRUB } & \nabla \text { LOCAL SHRUB } \\ \nabla \text { GRASS } & \nabla \text { LOCAL GRASS } \\ \nabla \text { FORBS } & \nabla \text { LOCAL FORBS } \\ \square \text { WETLAND } & \square \text { LOCAL WETLAND }\end{array}$

URL: www.afnn.org

Meadow Beauty Nursery

5782 Ranches Rd

Lake Worth, FL

33463

PHONE: (561) 966-6848

$\begin{array}{ll}\nabla \text { TREE } & \nabla \text { LOCAL TREE } \\ \nabla \text { SHRUB } & \nabla \text { LOCAL SHRUB } \\ \nabla \text { GRASS } & \nabla \text { LOCAL GRASS } \\ \nabla \text { FORBS } & \nabla \text { LOCAL FORBS } \\ \square \text { WETLAND } & \square \text { LOCAL WETLAND }\end{array}$

URL: www.afnn.org

Mesozoic Nurseries, Inc.

7667 Park Lane W.

Lake Worth, FL

33467

PHONE: (561) 967-2630

$\begin{array}{ll}\nabla \text { TREE } & \nabla \text { LOCAL TREE } \\ \nabla \text { SHRUB } & \nabla \text { LOCAL SHRUB } \\ \nabla \text { GRASS } & \nabla \text { LOCAL GRASS } \\ \nabla \text { FORBS } & \nabla \text { LOCAL FORBS } \\ \nabla \text { WETLAND } & \nabla \text { LOCAL WETLAND }\end{array}$

URL: http:/Www.afnn.org

Sunco

2269 2nd Ave. N.

Lake Worth, FL

33461

PHONE: (561) 586-7402

$\begin{array}{ll}\nabla \text { TREE } & \square \text { LOCAL TREE } \\ \square \text { SHRUB } & \square \text { LOCAL SHRUB } \\ \square \text { GRASS } & \square \text { LOCAL GRASS } \\ \square \text { FORBS } & \square \text { LOCAL FORBS } \\ \square \text { WETLAND } & \square \text { LOCAL WETLAND }\end{array}$

URL: http://Ww'w. suncotrees.com

Sundance Ornamentals

10689 Heritage Blvd

Lake Worth, FL

33467-672

PHONE: (561) 965-1344

$\begin{array}{ll}\nabla \text { TREE } & \nabla \text { LOCAL TREE } \\ \nabla \text { SHRUB } & \square \text { LOCAL SHRUB } \\ \nabla \text { GRASS } & \square \text { LOCAL GRASS } \\ \nabla \text { FORBS } & \nabla \text { LOCAL FORBS } \\ \square \text { WETLAND } & \square \text { LOCAL WETLAND }\end{array}$

URL: www.afnn.org 
Lakeland

Florida Native Flora, Inc

PO Box 2291

Lakeland, FL

33806

PHONE: (863) 853-8695

$\begin{array}{ll}\nabla \text { TREE } & \square \text { LOCAL TREE } \\ \square \text { SHRUB } & \square \text { LOCAL SHRUB } \\ \nabla \text { GRASS } & \square \text { LOCAL GRASS } \\ \nabla \text { FORBS } & \square \text { LOCAL FORBS } \\ \square \text { WETLAND } & \square \text { LOCAL WETLAND }\end{array}$

URL: www.afnn.org

Largo

Wilcox Nursery

12501 Indian Rocks Road

$\nabla$ TREE

$\checkmark$ SHRUB

$\square$ GRASS

33774

PHONE: (727) 595-2073

$\checkmark$ FORBS

$\square$ WETLAND

$\nabla$ LOCAL TREE

$\checkmark$ LOCAL SHRUB

$\checkmark$ LOCAL GRASS

$\checkmark$ LOCAL FORBS

$\square$ LOCAL WETLAND

URL: www.wilcoxnursery.com

Lee

Superior Trees, Inc.

Box 9325

Lee, FL

32059

PHONE: (850) 971-5159

URL: none currently available

\section{Loxahatchee}

$3 E$ Tree Farms and Wetland Nursery

$\begin{array}{ll}\nabla \text { TREE } & \nabla \text { LOCAL TREE } \\ \nabla \text { SHRUB } & \nabla \text { LOCAL SHRUB } \\ \nabla \text { GRASS } & \nabla \text { LOCAL GRASS } \\ \nabla \text { FORBS } & \nabla \text { LOCAL FORBS } \\ \nabla \text { WETLAND } & \nabla \text { LOCAL WETLAND }\end{array}$

$\checkmark$ WETLAND

$\checkmark$ LOCAL WETLAND
POB 476

Loxahatchee, FL

33470

PHONE: (561) 798-2150

URL: http://afnn.org

Carl Bates Indigenous Plants

16956 Hollowtree Ln

Loxahatchee, $\mathrm{FL}$

33470-501

PHONE: (561) 358-7480

$\begin{array}{ll}\nabla \text { TREE } & \checkmark \text { LOCAL TREE } \\ \nabla \text { SHRUB } & \checkmark \text { LOCAL SHRUB } \\ \nabla \text { GRASS } & \checkmark \text { LOCAL GRASS } \\ \nabla \text { FORBS } & \nabla \text { LOCAL FORBS } \\ \nabla \text { WETLAND } & \checkmark \text { LOCAL WETLAND }\end{array}$

$\checkmark$ TREE

$\square$ SHRUB

$\nabla$ GRASS

$\checkmark$ FORBS

$\checkmark$ WETLAND

$\checkmark$ LOCAL TREE

$\square$ LOCAL SHRUB

$\nabla$ LOCAL GRASS

$\checkmark$ LOCAL FORBS

$\checkmark$ LOCAL WETLAND

URL: http://afnn.org

\section{D.R. Bates Seeds}

P.O. Box 68

Loxahatchee, FL

33470

PHONE: (561) 790-3246

URL: http:/Www.drbates.com

$\begin{array}{ll}\square \text { TREE } & \square \text { LOCAL TREE } \\ \square \text { SHRUB } & \square \text { LOCAL SHRUB } \\ \square \text { GRASS } & \square \text { LOCAL GRASS } \\ \square \text { FORBS } & \square \text { LOCAL FORBS } \\ \square \text { WETLAND } & \square \text { LOCAL WETLAND }\end{array}$


EnviroGlades, Inc.

PO Box 476

Loxahatchee, FL

33470

PHONE: (561) 798-4995

$\begin{array}{ll}\nabla \text { TREE } & \nabla \text { LOCAL TREE } \\ \nabla \text { SHRUB } & \nabla \text { LOCAL SHRUB } \\ \nabla \text { GRASS } & \nabla \text { LOCAL GRASS } \\ \nabla \text { FORBS } & \nabla \text { LOCAL FORBS } \\ \nabla \text { WETLAND } & \nabla \text { LOCAL WETLAND }\end{array}$

URL: none currently available

Southern Native Nursery, Inc.

16351 Van Gogh Rd

Loxahatchee, FL

33470

PHONE: (561) 798-1172

$\begin{array}{ll}\nabla \text { TREE } & \nabla \text { LOCAL TREE } \\ \nabla \text { SHRUB } & \nabla \text { LOCAL SHRUB } \\ \nabla \text { GRASS } & \boldsymbol{\nabla} \text { LOCAL GRASS } \\ \square \text { FORBS } & \square \text { LOCAL FORBS } \\ \square \text { WETLAND } & \square \text { LOCAL WETLAND }\end{array}$

URL: www.afnn.org

Lutz

Florida Native Nursery

17711 Livingston Ave

Lutz, FL

33559

$\checkmark$ TREE $\quad \boldsymbol{V}$ LOCAL TREE

$\checkmark$ SHRUB

$\checkmark$ LOCAL SHRUB

PHONE: (813) 920-9613

$\nabla$ GRASS $\quad \nabla$ LOCAL GRASS

$\square$ FORBS $\square$ LOCAL FORBS

$\square$ WETLAND $\square$ LOCAL WETLAND

URL: none currently available

Mayo

Central Florida Lands \& Timber, Inc

Rt. 1, Box 899"

Mayo, FL

32066

PHONE: (386) 294-1211

$\boldsymbol{v}$ TREE $\quad \boldsymbol{v}$ LOCAL TREE

$\square$ SHRUB $\square$ LOCAL SHRUB

$\square$ GRASS $\square$ LOCAL GRASS

$\square$ FORBS $\square$ LOCAL FORBS

$\checkmark$ WETLAND $\checkmark$ LOCAL WETLAND

URL: none currently available

\section{Melrose}

Chiappini Farm Native Nursery

POB 436

Melrose, FL

32666-043

PHONE: (800) 293-5413

$\begin{array}{ll}\nabla \text { TREE } & \square \text { LOCAL TREE } \\ \nabla \text { SHRUB } & \nabla \text { LOCAL SHRUB } \\ \square \text { GRASS } & \square \text { LOCAL GRASS } \\ \square \text { FORBS } & \square \text { LOCAL FORBS } \\ \square \text { WETLAND } & \square \text { LOCAL WETLAND }\end{array}$

URL: http://WWw atlantic.net $/ \sim$ dchiapin

Miami

A Hidden Garden

6901 SW 105th St

Miami, FL

33183

PHONE: (305) 272-0150

$\begin{array}{ll}\square \text { TREE } & \square \text { LOCAL TREE } \\ \square \text { SHRUB } & \square \text { LOCAL SHRUB } \\ \square \text { GRASS } & \square \text { LOCAL GRASS } \\ \square \text { FORBS } & \square \text { LOCAL FORBS } \\ \square \text { WETLAND } & \square \text { LOCAL WETLAND }\end{array}$

URL: none currently available 
Action Theory Landscaping \& Nursery 12995 226th St

Miami, FL

33143

PHONE: (305) 257-2244

URL: http:/Www plantiful.com/actiontheory

\section{Peltons Nursery}

PO Box 560912

Miami, FL

33256-091

PHONE: (305) 447-7667

URL: www.peltonsnurseries.com

Plant Doctor, Inc.

15195 S.W. 192nd St.

Miami, FL

33187

PHONE: (305) 255-4533

URL: none currently available

Sturon, Inc.

14260 SW 136th St, \#15

Miami, FL

33186

PHONE: (305) 232-9005

URL: none currently available

Vila \& Son Nursery Corp.

20451 SW 216th St

Miami, FL

33170

PHONE: (305) 245-2055

URL: www.vila-n-son.com

$\begin{array}{ll}\nabla \text { TREE } & \square \text { LOCAL TREE } \\ \nabla \text { SHRUB } & \square \text { LOCAL SHRUB } \\ \square \text { GRASS } & \square \text { LOCAL GRASS } \\ \square \text { FORBS } & \square \text { LOCAL FORBS } \\ \nabla \text { WETLAND } & \square \text { LOCAL WETLAND }\end{array}$

$\begin{array}{ll}\nabla \text { TREE } & \square \text { LOCAL TREE } \\ \square \text { SHRUB } & \square \text { LOCAL SHRUB } \\ \square \text { GRASS } & \square \text { LOCAL GRASS } \\ \square \text { FORBS } & \square \text { LOCAL FORBS } \\ \square \text { WETLAND } & \square \text { LOCAL WETLAND }\end{array}$

$\checkmark$ TREE

$\checkmark$ SHRUB

$\checkmark$ LOCAL TREE

$\checkmark$ LOCAL SHRUB

$\square$ GRASS

$\square$ FORBS

$\square$ LOCAL GRASS

$\square$ LOCAL FORBS

$\square$ WETLAND $\square$ LOCAL WETLAND $\checkmark$ TREE $\quad \square$ LOCAL TREE

$\checkmark$ SHRUB $\square$ LOCAL SHRUB

$\nabla$ GRASS $\square$ LOCAL GRASS

$\checkmark$ FORBS $\quad \square$ LOCAL FORBS

$\square$ WETLAND $\square$ LOCAL WETLAND

\section{Micanopy}

Simanton Farms Nursery

22049 NW 75 th Ave

Micanopy, FL

32667

PHONE: (352) 591-2271

$\begin{array}{ll}\nabla \text { TREE } & \nabla \text { LOCAL TREE } \\ \nabla \text { SHRUB } & \nabla \text { LOCAL SHRUB } \\ \nabla \text { GRASS } & \square \text { LOCAL GRASS } \\ \square \text { FORBS } & \square \text { LOCAL FORBS } \\ \square \text { WETLAND } & \square \text { LOCAL WETLAND }\end{array}$

URL: none currently available

Urban Forestry Services

301 W Seminary St

Micanopy, FL

32667

PHONE: (352) 466-3919

$\begin{array}{ll}\nabla \text { TREE } & \square \text { LOCAL TREE } \\ \square \text { SHRUB } & \square \text { LOCAL SHRUB } \\ \square \text { GRASS } & \square \text { LOCAL GRASS } \\ \square \text { FORBS } & \square \text { LOCAL FORBS } \\ \square \text { WETLAND } & \square \text { LOCAL WETLAND }\end{array}$

URL: www.afnn.org

$\begin{array}{ll}\nabla \text { TREE } & \square \text { LOCAL TREE } \\ \square \text { SHRUB } & \square \text { LOCAL SHRUB } \\ \square \text { GRASS } & \square \text { LOCAL GRASS } \\ \square \text { FORBS } & \square \text { LOCAL FORBS } \\ \square \text { WETLAND } & \square \text { LOCAL WETLAND }\end{array}$


Milton

Rancho la Orquidea Inc

1124 Pearson Rd

Milton. FL

32583-913

$\checkmark$ TREE

$\checkmark$ SHRUB

$\checkmark$ GRASS

$\nabla$ FORBS

$\checkmark$ WETLAND

$\checkmark$ LOCAL SHRUB

PHONE: (850) 983-8948

URL: www.rancholaorquidea.com

Southern Native Plants, Inc.

6322 Mary Kitchens Rd

Milton, FL

32583

PHONE: (850) 983-9121

$\begin{array}{ll}\nabla \text { TREE } & \nabla \text { LOCAL TREE } \\ \nabla \text { SHRUB } & \nabla \text { LOCAL SHRUB } \\ \nabla \text { GRASS } & \nabla \text { LOCAL GRASS } \\ \nabla \text { FORBS } & \nabla \text { LOCAL FORBS } \\ \square \text { WETLAND } & \square \text { LOCAL WETLAND }\end{array}$

URL: www.afnn.org

Minneola

Matlack Tree Farm

PO Box 67

Minneola, FL

34755

PHONE: (352) 406-9735

$\checkmark$ LOCAL GRASS

$\nabla$ LOCAL FORBS

$\checkmark$ LOCAL WETLAND

URL: www.afnn.org

\section{Monticello}

Apalachee Native Nursery

12 Hayfield Spur

Monticello, FL

32344

PHONE: (850) 997-8976

$\begin{array}{ll}\nabla \text { TREE } & \square \text { LOCAL TREE } \\ \square \text { SHRUB } & \square \text { LOCAL SHRUB } \\ \square \text { GRASS } & \square \text { LOCAL GRASS } \\ \square \text { FORBS } & \square \text { LOCAL FORBS } \\ \square \text { WETLAND } & \square \text { LOCAL WETLAND }\end{array}$

URL: http://wantplants.com

Morriston

Marshall Tree Farm

17350 SE 65th St

Morriston, FL

32668

PHONE: (800) 786-1422

$\begin{array}{ll}\nabla \text { TREE } & \nabla \text { LOCAL TREE } \\ \nabla \text { SHRUB } & \nabla \text { LOCAL SHRUB } \\ \nabla \text { GRASS } & \nabla \text { LOCAL GRASS } \\ \nabla \text { FORBS } & \nabla \text { LOCAL FORBS } \\ \nabla \text { WETLAND } & \nabla \text { LOCAL WETLAND }\end{array}$

URL: www.marshalltrees.com

\section{Myakka City}

McKeithen Growers, Inc.

24005 71st Ave E

Myakka City, FL

34251

PHONE: (941) 322-2397

$\begin{array}{ll}\nabla \text { TREE } & \checkmark \text { LOCAL TREE } \\ \square \text { SHRUB } & \square \text { LOCAL SHRUB } \\ \square \text { GRASS } & \square \text { LOCAL GRASS } \\ \square \text { FORBS } & \square \text { LOCAL FORBS } \\ \square \text { WETLAND } & \square \text { LOCAL WETLAND }\end{array}$

URL: www.afnn.org

$\begin{array}{ll}\nabla \text { TREE } & \square \text { LOCAL TREE } \\ \square \text { SHRUB } & \square \text { LOCAL SHRUB } \\ \square \text { GRASS } & \square \text { LOCAL GRASS } \\ \square \text { FORBS } & \square \text { LOCAL FORBS } \\ \square \text { WETLAND } & \square \text { LOCAL WETLAND }\end{array}$


Naples

Superior Plant Co.

510 Frangipani Ave.

Naples, FL

34117

PHONE: (941) 455-1560

URL: none currently available

Trans Gro (Superior Plant Company Division)

510 Frangipani Ave

Naples, FL

34117

PHONE: (239) 657-6141

URL: www.afnn.org

Wylde Thyme Hammock

2861 Sherman Ave

Naples, FL

34120

PHONE: (941) 352-9115

URL: www.afnn.org

New Smyrna Beach

Beemans Nursery, Inc.

3881 S. Nova Rd.

New Smyrna Beach, FL

32168

PHONE: (386) 428-8889

URL: http://beemansnursery.com

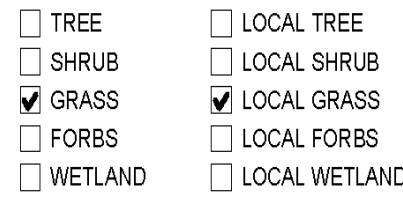

$\begin{array}{ll}\nabla \text { TREE } & \square \text { LOCAL TREE } \\ \square \text { SHRUB } & \square \text { LOCAL SHRUB } \\ \nabla \text { GRASS } & \square \text { LOCAL GRASS } \\ \nabla \text { FORBS } & \square \text { LOCAL FORBS } \\ \square \text { WETLAND } & \square \text { LOCAL WETLAND }\end{array}$

$\checkmark$ TREE

$\square$ SHRUB

$\checkmark$ GRASS

$\square$ FORBS

$\square$ WETLAND

$\checkmark$ LOCAL TREE

$\square$ LOCAL SHRUB

$\bar{\nabla}$ LOCAL GRASS

$\square$ LOCAL FORBS

$\square$ LOCAL WETLAND

$\square$ WETLAND $\square$ LOCAL WETLAND 


\section{Ocala}

Bent Tree Farm, Inc. 4273 N.W.CR 225-A

Ocala, FL

34482

PHONE: (352) 732-9564

URL: none currently available

Greenbriar Nurseries, Inc. Box 5189

Ocala, FL

32678

PHONE: (352) 489-0133

$\begin{array}{ll}\square \text { TREE } & \square \text { LOCAL TREE } \\ \square \text { SHRUB } & \square \text { LOCAL SHRUB } \\ \square \text { GRASS } & \square \text { LOCAL GRASS } \\ \square \text { FORBS } & \square \text { LOCAL FORBS } \\ \square \text { WETLAND } & \square \text { LOCAL WETLAND }\end{array}$

URL: http://Nww greenbriarnurseries.com

Old Town

Old Town Native Nursery

HC4, Box 68-351-A

Old Town, FL

32680

PHONE: (352) 498-2907

$\begin{array}{ll}\nabla \text { TREE } & \square \text { LOCAL TREE } \\ \square \text { SHRUB } & \square \text { LOCAL SHRUB } \\ \square \text { GRASS } & \square \text { LOCAL GRASS } \\ \square \text { FORBS } & \square \text { LOCAL FORBS } \\ \square \text { WETLAND } & \square \text { LOCAL WETLAND }\end{array}$

URL: none currently available

\section{Orange Park}

Northeast Florida Native Nursery

1524 Smith St

Orange Park, FL

32073

PHONE: (904) 264-6699

$\begin{array}{ll}\nabla \text { TREE } & \nabla \text { LOCAL TREE } \\ \nabla \text { SHRUB } & \nabla \text { LOCAL SHRUB } \\ \nabla \text { GRASS } & \square \text { LOCAL GRASS } \\ \nabla \text { FORBS } & \checkmark \text { LOCAL FORBS } \\ \square \text { WETLAND } & \square \text { LOCAL WETLAND }\end{array}$

URL: www.afnn.org

Oxford

Brown \& Brown Seed

13992 N. U.S. Hwy 31

Oxford, FL

34484

PHONE: (352) 245-3222

$\begin{array}{ll}\nabla \text { TREE } & \square \text { LOCAL TREE } \\ \nabla \text { SHRUB } & \square \text { LOCAL SHRUB } \\ \square \text { GRASS } & \square \text { LOCAL GRASS } \\ \square \text { FORBS } & \square \text { LOCAL FORBS } \\ \square \text { WETLAND } & \square \text { LOCAL WETLAND }\end{array}$

URL: none currently available

\section{Pahokee}

Oasis Tree Farms

PO Box 539

Pahokee, FL

33476

PHONE: (561) 924-2400

$\begin{array}{ll}\square \text { TREE } & \square \text { LOCAL TREE } \\ \square \text { SHRUB } & \square \text { LOCAL SHRUB } \\ \square \text { GRASS } & \square \text { LOCAL GRASS } \\ \square \text { FORBS } & \square \text { LOCAL FORBS } \\ \square \text { WETLAND } & \square \text { LOCAL WETLAND }\end{array}$

URL: none currently available

$\begin{array}{ll}\square \text { TREE } & \checkmark \text { LOCAL TREE } \\ \square \text { SHRUB } & \square \text { LOCAL SHRUB } \\ \square \text { GRASS } & \square \text { LOCAL GRASS } \\ \square \text { FORBS } & \square \text { LOCAL FORBS } \\ \square \text { WETLAND } & \square \text { LOCAL WETLAND }\end{array}$


Palmetto

Horticultural Systems

Golf Course Road

Palmetto, FL

34221

$\checkmark$ TREE

$\checkmark$ SHRUB

$\checkmark$ GRASS

$\checkmark$ FORBS

$\checkmark$ WETLAND

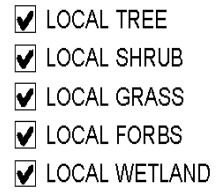

URL: http:/Wwww.ecogroup.com

Parrish

Green Seasons Nursery

P.O. Box 539

Parrish, FL

34219

PHONE: (941) 776-1605

$\begin{array}{ll}\square \text { TREE } & \square \text { LOCAL TREE } \\ \square \text { SHRUB } & \square \text { LOCAL SHRUB } \\ \square \text { GRASS } & \square \text { LOCAL GRASS } \\ \nabla \text { FORBS } & \square \text { LOCAL FORBS } \\ \square \text { WETLAND } & \square \text { LOCAL WETLAND }\end{array}$

URL: none currently available

Sweet Bay Nursery

10824 Erie Rd

Parrish, FL

34219

PHONE: (941) 776-0501

$\begin{array}{ll}\nabla \text { TREE } & \square \text { LOCAL TREE } \\ \nabla \text { SHRUB } & \square \text { LOCAL SHRUB } \\ \nabla \text { GRASS } & \square \text { LOCAL GRASS } \\ \square \text { FORBS } & \square \text { LOCAL FORBS } \\ \square \text { WETLAND } & \square \text { LOCAL WETLAND }\end{array}$

URL: wWw.afnn.org

\section{Penny Farms}

Shadowlawn Nursery

PO Box 515

Penny Farms, FL

32079-051

PHONE: (904) 269-5857

$\begin{array}{ll}\nabla \text { TREE } & \square \text { LOCAL TREE } \\ \square \text { SHRUB } & \square \text { LOCAL SHRUB } \\ \square \text { GRASS } & \square \text { LOCAL GRASS } \\ \square \text { FORBS } & \square \text { LOCAL FORBS } \\ \square \text { WETLAND } & \square \text { LOCAL WETLAND }\end{array}$

URL: www.afnn.org

\section{Pensacola}

The Echo Center

1055 Echo Circle

Pensacola, FL

32514

PHONE: (850) 478-1985

$\begin{array}{ll}\nabla \text { TREE } & \nabla \text { LOCAL TREE } \\ \nabla \text { SHRUB } & \checkmark \text { LOCAL SHRUB } \\ \nabla \text { GRASS } & \nabla \text { LOCAL GRASS } \\ \nabla \text { FORBS } & \nabla \text { LOCAL FORBS } \\ \nabla \text { WETLAND } & \checkmark \text { LOCAL WETLAND }\end{array}$

URL: none currently available

Plant City

RSS Field Services, Inc.

POB 549

Plant City, FL

33564

PHONE: (813) 754-7160

$\begin{array}{ll}\nabla \text { TREE } & \square \text { LOCAL TREE } \\ \square \text { SHRUB } & \square \text { LOCAL SHRUB } \\ \nabla \text { GRASS } & \square \text { LOCAL GRASS } \\ \square \text { FORBS } & \square \text { LOCAL FORBS } \\ \square \text { WETLAND } & \square \text { LOCAL WETLAND }\end{array}$

URL: www.afnn.org 
Suncoast Plant Nursery

5512 W Thonotosassa Rd

$\checkmark$ TREE $\quad \nabla$ LOCAL TREE

Plant City, FL

33565

PHONE: (813) 986-3713

$\nabla$ SHRUB $\quad \bar{v}$ LOCAL SHRUB

$\square$ GRASS $\square$ LOCAL GRASS

$\square$ FORBS $\square$ LOCAL FORBS

$\checkmark$ WETLAND $\square$ LOCAL WETLAND

URL: none currently available

Ruskin

Sun City Tree Farm \& Nursery

270130 th ST SE

Ruskin, $\mathrm{FL}$

33570

PHONE: (813) 645-9527

$\begin{array}{ll}\square \text { TREE } & \square \text { LOCAL TREE } \\ \square \text { SHRUB } & \square \text { LOCAL SHRUB } \\ \square \text { GRASS } & \square \text { LOCAL GRASS } \\ \square \text { FORBS } & \square \text { LOCAL FORBS } \\ \square \text { WETLAND } & \square \text { LOCAL WETLAND }\end{array}$

URL: www.afnn.org

San Antonio

All Native, LLC

PO Box 1045

San Antonio, FL

33576

PHONE: (352) 588-3687

$\checkmark$ TREE LOCAL TREE

$\nabla$ SHRUB $\quad \nabla$ LOCAL SHRUB

$\nabla$ GRASS $\quad \nabla$ LOCAL GRASS

$\checkmark$ FORBS $\quad \nabla$ LOCAL FORBS

$\nabla$ WETLAND $\nabla$ LOCAL WETLAND

URL: www.allnativeflora.com

CFNF/All Native

PO Box 1045

San Antonio, FL

33576

PHONE: (352) 588-3687

$\begin{array}{ll}\nabla \text { TREE } & \checkmark \text { LOCAL TREE } \\ \nabla \text { SHRUB } & \checkmark \text { LOCAL SHRUB } \\ \square \text { GRASS } & \checkmark \text { LOCAL GRASS } \\ \square \text { FORBS } & \square \text { LOCAL FORBS } \\ \square \text { WETLAND } & \checkmark \text { LOCAL WETLAND }\end{array}$

URL: http://afnn.org

\section{Sanibel}

Native Plant Nursery

Box 839

$\nabla$ TREE $\quad \nabla$ LOCAL TREE

Sanibel, FL

$\nabla$ SHRUB

$\checkmark$ LOCAL SHRUB

33957

$\checkmark$ GRASS $\quad \nabla$ LOCAL GRASS

PHONE: (941) 472-1932

$\checkmark$ FORBS $\quad \nabla$ LOCAL FORBS

$\nabla$ WETLAND $\nabla$ LOCAL WETLAND

URL: http:/WWWw.Sccf.org

\section{Santa Rosa Beach}

The Gourd Garden \& Curiosity Shop

4808 E County Rd 30-A,

Santa Rosa Beach, FL

32459

PHONE: (850) 231-2007

$\begin{array}{ll}\nabla \text { TREE } & \square \text { LOCAL TREE } \\ \nabla \text { SHRUB } & \nabla \text { LOCAL SHRUB } \\ \nabla \text { GRASS } & \square \text { LOCAL GRASS } \\ \square \text { FORBS } & \square \text { LOCAL FORBS } \\ \square \text { WETLAND } & \square \text { LOCAL WETLAND }\end{array}$

URL: www.gourdgarden.com 


\section{Sarasota}

Aquatic Plants of Florida

1491 Second St, Ste C-1

Sarasota, FL

34236

PHONE: (941) 952-9886

URL: http:/Wwww.aquaticplantsofflorida.com

\section{Coastal Aquatic Services, Inc.}

P.O. Box 37525

Sarasota, FL

34278

PHONE: (941) 378-5320

$\begin{array}{ll}\nabla \text { TREE } & \nabla \text { LOCAL TREE } \\ \nabla \text { SHRUB } & \nabla \text { LOCAL SHRUB } \\ \nabla \text { GRASS } & \nabla \text { LOCAL GRASS } \\ \nabla \text { FORBS } & \nabla \text { LOCAL FORBS } \\ \nabla \text { WETLAND } & \nabla \text { LOCAL WETLAND }\end{array}$

URL: none currently available

Florida Native Plants

730 Myakka Rd

$\nabla$ TREE $\quad \nabla$ LOCAL TREE

Sarasota, $\mathrm{FL}$

34240

PHONE: (941) 322-1915

$\checkmark$ SHRUB $\nabla$ LOCAL SHRUB

$\nabla$ GRASS $\quad \nabla$ LOCAL GRASS

$\nabla$ FORBS $\quad \nabla$ LOCAL FORBS

$\checkmark$ WETLAND $\square$ LOCAL WETLAND

URL: http:/flloridanativeplants.com

\section{Scottsmoor}

American Native Products

3455 Johns Road

Scottsmoor, FL

32755

PHONE: (321) 383-1967

$\begin{array}{ll}\square \text { TREE } & \square \text { LOCAL TREE } \\ \nabla \text { SHRUB } & \nabla \text { LOCAL SHRUB } \\ \square \text { GRASS } & \square \text { LOCAL GRASS } \\ \square \text { FORBS } & \square \text { LOCAL FORBS } \\ \square \text { WETLAND } & \square \text { LOCAL WETLAND }\end{array}$

URL: none currently available

\section{Sebring}

C M Payne \& Son Seed Co.

9410 Payne Rd.

Sebring. FL

33872

PHONE: (863) 385-4642

$\begin{array}{ll}\square \text { TREE } & \square \text { LOCAL TREE } \\ \square \text { SHRUB } & \square \text { LOCAL SHRUB } \\ \square \text { GRASS } & \square \text { LOCAL GRASS } \\ \square \text { FORBS } & \square \text { LOCAL FORBS } \\ \square \text { WETLAND } & \square \text { LOCAL WETLAND }\end{array}$

URL: none currently available

Finders Keepers Plants \& Broker Inc.

5031 Whippoorwill Rd

Sebring. FL

33875-638

$\begin{array}{ll}\square \text { TREE } & \square \text { LOCAL TREE } \\ \square \text { SHRUB } & \square \text { LOCAL SHRUB } \\ \nabla \text { GRASS } & \square \text { LOCAL GRASS } \\ \nabla \text { FORBS } & \square \text { LOCAL FORBS } \\ \square \text { WETLAND } & \square \text { LOCAL WETLAND }\end{array}$

URL: www.finderskeepersplants.com 


\section{St. Augustine}

Southern Horticulture

1690 A1AS

St. Augustine, FL

32080

PHONE: (904) 471-0440

URL: www.afnn.org

$\begin{array}{ll}\square \text { TREE } & \square \text { LOCAL TREE } \\ \square \text { SHRUB } & \square \text { LOCAL SHRUB } \\ \nabla \text { GRASS } & \square \text { LOCAL GRASS } \\ \nabla \text { FORBS } & \square \text { LOCAL FORBS } \\ \nabla \text { WETLAND } & \square \text { LOCAL WETLAND }\end{array}$

$\nabla$ WETLAND $\nabla$ LOCAL WETLAND

\section{St. Cloud}

The Liner Farm

Box 701369

St. Cloud, FL

34770

PHONE: (407) 892-1484

URL: none currently available

$\begin{array}{ll}\nabla \text { TREE } & \nabla \text { LOCAL TREE } \\ \nabla \text { SHRUB } & \nabla \text { LOCAL SHRUB } \\ \nabla \text { GRASS } & \nabla \text { LOCAL GRASS } \\ \nabla \text { FORBS } & \nabla \text { LOCAL FORBS } \\ \nabla \text { WETLAND } & \nabla \text { LOCAL WETLAND }\end{array}$

$\checkmark$ WETLAND

\section{Tallahassee}

Florida Division of Forestry

3125 Conner Blvd

Tallahassee, FL

32399-165

PHONE: (850) 488-4274

URL: http://Wwww.fl-dof.com/

Native Nurseries

1661 Centerville Rd.

Tallahassee, FL

32308

PHONE: (850) 386-8882

$\begin{array}{ll}\square \text { TREE } & \square \text { LOCAL TREE } \\ \square \text { SHRUB } & \square \text { LOCAL SHRUB } \\ \square \text { GRASS } & \square \text { LOCAL GRASS } \\ \square \text { FORBS } & \square \text { LOCAL FORBS } \\ \square \text { WETLAND } & \square \text { LOCAL WETLAND }\end{array}$

URL: http://Www nativenurseries.com

Tampa

Ecological Consultants, Inc. 5121 Ehrlich Rd, Ste 103A,

Tampa, FL

33624

PHONE: (813) 264-5859

$\begin{array}{ll}\nabla \text { TREE } & \nabla \text { LOCAL TREE } \\ \nabla \text { SHRUB } & \nabla \text { LOCAL SHRUB } \\ \nabla \text { GRASS } & \checkmark \text { LOCAL GRASS } \\ \nabla \text { FORBS } & \checkmark \text { LOCAL FORBS } \\ \square \text { WETLAND } & \checkmark \text { LOCAL WETLAND }\end{array}$

URL: www.afnn.org

Treemart

12505 N Nebraska Ave

Tampa, FL

33612

PHONE: (800) 664-4006

$\begin{array}{ll}\square \text { TREE } & \square \text { LOCAL TREE } \\ \square \text { SHRUB } & \square \text { LOCAL SHRUB } \\ \square \text { GRASS } & \square \text { LOCAL GRASS } \\ \square \text { FORBS } & \square \text { LOCAL FORBS } \\ \square \text { WETLAND } & \square \text { LOCAL WETLAND }\end{array}$

URL: www.treemart.com

$\begin{array}{ll}\nabla \text { TREE } & \square \text { LOCAL TREE } \\ \square \text { SHRUB } & \square \text { LOCAL SHRUB } \\ \nabla \text { GRASS } & \square \text { LOCAL GRASS } \\ \square \text { FORBS } & \square \text { LOCAL FORBS } \\ \square \text { WETLAND } & \square \text { LOCAL WETLAND }\end{array}$


Taveras

Native Creations

28200 Tammi Dr

Taveras, FL

32778

PHONE: (352) 343-3854

$\begin{array}{ll}\nabla \text { TREE } & \square \text { LOCAL TREE } \\ \square \text { SHRUB } & \square \text { LOCAL SHRUB } \\ \nabla \text { GRASS } & \square \text { LOCAL GRASS } \\ \nabla \text { FORBS } & \square \text { LOCAL FORBS } \\ \nabla \text { WETLAND } & \square \text { LOCAL WETLAND }\end{array}$

URL: www.afnn.org

Tavernier

Florida Keys Native Nursery, Inc.

102 Mohawk St.

$\nabla$ TREE $\quad \nabla$ LOCAL TREE

Tavernier, FL

$\checkmark$ SHRUB $\quad \nabla$ LOCAL SHRUB

33070

$\checkmark$ GRASS $\quad \nabla$ LOCAL GRASS

PHONE: (305) 852-2636

$\nabla$ FORBS $\quad \nabla$ LOCAL FORBS

$\square$ WETLAND $\square$ LOCAL WETLAND

URL: http://Www.afnn.org

Terra Ceia

Native Plant Brokerage

PO Box 206

$\checkmark$ TREE $\quad \nabla$ LOCAL TREE

Terra Ceia, FL

34250

$\square$ SHRUB $\square$ LOCAL SHRUB

PHONE: (941) 723-5091

$\nabla$ GRASS $\quad \nabla$ LOCAL GRASS

$\checkmark$ FORBS $\quad \nabla$ LOCAL FORBS

$\square$ WETLAND $\square$ LOCAL WETLAND

URL: www.afnn.org

Terra Ceia Island

Hard Scrabble Farm

Box 281

$\nabla$ TREE $\quad \boldsymbol{\nabla}$ LOCAL TREE

Terra Ceia Island, FL

34250

PHONE: (941) 722-0414

$\nabla$ SHRUB $\quad \nabla$ LOCAL SHRUB

$\nabla$ GRASS $\quad \nabla$ LOCAL GRASS

$\checkmark$ FORBS $\quad \nabla$ LOCAL FORBS

$\checkmark$ WETLAND $\checkmark$ LOCAL WETLAND

URL: http://Www afinn.org

\section{W. Melbourne}

Maple Street Natives

2395 Maple Street

W. Melbourne, FL

32904

PHONE: (321) 729-6857

$\begin{array}{ll}\nabla \text { TREE } & \nabla \text { LOCAL TREE } \\ \nabla \text { SHRUB } & \nabla \text { LOCAL SHRUB } \\ \nabla \text { GRASS } & \square \text { LOCAL GRASS } \\ \nabla \text { FORBS } & \square \text { LOCAL FORBS } \\ \square \text { WETLAND } & \square \text { LOCAL WETLAND }\end{array}$

URL: www.maplestreetnatives.com

\section{West Palm Beach}

Butterflys Are Free Nursery, Inc. 1283 Ranchette Rd.

West Palm Beach, FL

33415

PHONE: (561) 683-3158

$\begin{array}{ll}\square \text { TREE } & \square \text { LOCAL TREE } \\ \square \text { SHRUB } & \square \text { LOCAL SHRUB } \\ \square \text { GRASS } & \square \text { LOCAL GRASS } \\ \square \text { FORBS } & \square \text { LOCAL FORBS } \\ \square \text { WETLAND } & \square \text { LOCAL WETLAND }\end{array}$

URL: none currently available 
Winter Garden

Biosphere Consulting, Inc.

14908 Tilden Rd

Winter Garden, FL

34787

PHONE: (407) 656-8277

$\begin{array}{ll}\nabla \text { TREE } & \nabla \text { LOCAL TREE } \\ \nabla \text { SHRUB } & \nabla \text { LOCAL SHRUB } \\ \nabla \text { GRASS } & \nabla \text { LOCAL GRASS } \\ \nabla \text { FORBS } & \nabla \text { LOCAL FORBS } \\ \nabla \text { WETLAND } & \nabla \text { LOCAL WETLAND }\end{array}$

URL: http://Nww biospherenursery.com

\section{Georgia}

Americus

\section{Plant Materials Center}

295 Morris Dr

Americus, GA

31709

PHONE: (229) 924-4499

$\begin{array}{ll}\square \text { TREE } & \square \text { LOCAL TREE } \\ \square \text { SHRUB } & \square \text { LOCAL SHRUB } \\ \square \text { GRASS } & \square \text { LOCAL GRASS } \\ \square \text { FORBS } & \square \text { LOCAL FORBS } \\ \square \text { WETLAND } & \square \text { LOCAL WETLAND }\end{array}$

URL: http://plant-materials.nrcs.usda.gov/gapmc/

Bellville

International Paper/ Bellville Super Tree Nusery

PO Box 56

Bellville, GA

30414

PHONE: (877) 833-4760

$\begin{array}{ll}\square \text { TREE } & \square \text { LOCAL TREE } \\ \square \text { SHRUB } & \square \text { LOCAL SHRUB } \\ \square \text { GRASS } & \square \text { LOCAL GRASS } \\ \square \text { FORBS } & \square \text { LOCAL FORBS } \\ \square \text { WETLAND } & \square \text { LOCAL WETLAND }\end{array}$

URL: none currently available

\section{Blue Ridge}

Lakewood Landscapes

148 Laurel Ridge Dr.

Blue Ridge, GA

30513

PHONE: (706) 632-5739

$\begin{array}{ll}\nabla \text { TREE } & \square \text { LOCAL TREE } \\ \nabla \text { SHRUB } & \square \text { LOCAL SHRUB } \\ \square \text { GRASS } & \square \text { LOCAL GRASS } \\ \nabla \text { FORBS } & \square \text { LOCAL FORBS } \\ \nabla \text { WETLAND } & \square \text { LOCAL WETLAND }\end{array}$

URL: none currently available

\section{Cataula}

Piedmont Growers

$\begin{array}{lll}\text { 1877 Hunter Rd } & \nabla \text { TREE } & \square \text { LOCAL TREE } \\ \text { Cataula, GA } & \nabla \text { SHRUB } & \square \text { LOCAL SHRUB } \\ 31804 & \square \text { GRASS } & \square \text { LOCAL GRASS } \\ \text { PHONE: (706) 596-9393 } & \square \text { FORBS } & \square \text { LOCAL FORBS } \\ & \square \text { WETLAND } & \square \text { LOCAL WETLAND }\end{array}$

URL: none currently available

\section{Chamblee}

Doublefile Nursery 3434 Blackburn Way

Chamblee, GA

30341

PHONE: (770) 614-8322

$\begin{array}{ll}\nabla \text { TREE } & \square \text { LOCAL TREE } \\ \square \text { SHRUB } & \square \text { LOCAL SHRUB } \\ \square \text { GRASS } & \square \text { LOCAL GRASS } \\ \square \text { FORBS } & \square \text { LOCAL FORBS } \\ \square \text { WETLAND } & \square \text { LOCAL WETLAND }\end{array}$

URL: none currently available 
Claxton

Spandle Nurseries

Box 125

Claxton, GA

30417

PHONE: (912) 739-1598

$\checkmark$ TREE

$\checkmark$ SHRUB

$\checkmark$ GRASS

$\nabla$ FORBS

$\checkmark$ LOCAL TREE

$\checkmark$ LOCAL SHRUB

URL: http://www.spandles.com

\section{Columbus}

Powell Propagators \& Nursery

6801 Warm Springs Rd

Columbus, GA

31909

$\square$ WETLAND

$\square$ LOCAL GRASS

$\square$ LOCAL FORBS

$\square$ LOCAL WETLAND

PHONE: (404) 568-1271

$\begin{array}{ll}\nabla \text { TREE } & \nabla \text { LOCAL TREE } \\ \square \text { SHRUB } & \nabla \text { LOCAL SHRUB } \\ \square \text { GRASS } & \square \text { LOCAL GRASS } \\ \square \text { FORBS } & \square \text { LOCAL FORBS } \\ \square \text { WETLAND } & \square \text { LOCAL WETLAND }\end{array}$

URL: none currently available

\section{Cordele}

Southern Christmas Tree Nursery

3452 Poteville Rd

Cordele, GA

31015

PHONE: (912) 273-5748

$\begin{array}{ll}\square \text { TREE } & \square \text { LOCAL TREE } \\ \square \text { SHRUB } & \square \text { LOCAL SHRUB } \\ \square \text { GRASS } & \square \text { LOCAL GRASS } \\ \square \text { FORBS } & \square \text { LOCAL FORBS } \\ \square \text { WETLAND } & \square \text { LOCAL WETLAND }\end{array}$

URL: none currently available

\section{Dearing}

McCorkle Nurseries

4904 Luckey's Bridge Rd SE

Dearing, GA

30808

PHONE: (706) 595-9702

$\begin{array}{ll}\nabla \text { TREE } & \square \text { LOCAL TREE } \\ \square \text { SHRUB } & \square \text { LOCAL SHRUB } \\ \square \text { GRASS } & \square \text { LOCAL GRASS } \\ \square \text { FORBS } & \square \text { LOCAL FORBS } \\ \square \text { WETLAND } & \square \text { LOCAL WETLAND }\end{array}$

URL: http://Www mccorklenurseries.com/

Decatur

Eco-Gardens

P.O. Box 1227

Decatur, GA

30031

PHONE: (404) 294-6468

$\begin{array}{ll}\square \text { TREE } & \square \text { LOCAL TREE } \\ \square \text { SHRUB } & \square \text { LOCAL SHRUB } \\ \square \text { GRASS } & \square \text { LOCAL GRASS } \\ \nabla \text { FORBS } & \square \text { LOCAL FORBS } \\ \square \text { WETLAND } & \square \text { LOCAL WETLAND }\end{array}$

URL: none currently available

$\begin{array}{lll}\text { Jackson } & & \\ \text { Adams-Briscoe Seed Co. } & & \\ 325 \text { E. Second St. } & \square \text { TREE } & \square \text { LOCAL TREE } \\ \text { Jackson, GA } & \square \text { SHRUB } & \square \text { LOCAL SHRUB } \\ 30233 & \square \text { GRASS } & \square \text { LOCAL GRASS } \\ \text { PHONE: (770) 775-7826 } & \square \text { FORBS } & \square \text { LOCAL FORBS } \\ & \square \text { WETLAND } & \square \text { LOCAL WETLAND }\end{array}$

URL: http://Www abseed.com 
Lavonia

Transplant Nursery

1584 Parkertown Rd.

Lavonia, GA

30553

PHONE: (706) 356-8947

URL: none currently available

\section{Lexington}

Goodness Grows, Inc.

332 Elberton St.

Lexington, GA

30648

PHONE: (706) 743-5055

$\begin{array}{ll}\square \text { TREE } & \square \text { LOCAL TREE } \\ \square \text { SHRUB } & \square \text { LOCAL SHRUB } \\ \square \text { GRASS } & \square \text { LOCAL GRASS } \\ \square \text { FORBS } & \square \text { LOCAL FORBS } \\ \square \text { WETLAND } & \square \text { LOCAL WETLAND }\end{array}$

URL: none currently available

Macon

Georgia Forestry Commission

P. O. Box 819

Macon, GA

31202-081

PHONE: (478) 751-3500

$\begin{array}{ll}\square \text { TREE } & \square \text { LOCAL TREE } \\ \square \text { SHRUB } & \square \text { LOCAL SHRUB } \\ \square \text { GRASS } & \square \text { LOCAL GRASS } \\ \square \text { FORBS } & \square \text { LOCAL FORBS } \\ \square \text { WETLAND } & \square \text { LOCAL WETLAND }\end{array}$

JRL: http://Www gfc, state.ga us/

Madison

Pennington Seed

PO Box 290

Madison, GA

30650

PHONE: (800) 285-7333

$\begin{array}{ll}\square \text { TREE } & \square \text { LOCAL TREE } \\ \square \text { SHRUB } & \square \text { LOCAL SHRUB } \\ \square \text { GRASS } & \square \text { LOCAL GRASS } \\ \square \text { FORBS } & \square \text { LOCAL FORBS } \\ \square \text { WETLAND } & \square \text { LOCAL WETLAND }\end{array}$

URL: www.penningtonseed.com

Monroe

Land Arts, Inc.

809 North Broad St.

Monroe, GA

30656-140

PHONE: (770) 267-4500

$\begin{array}{ll}\square \text { TREE } & \square \text { LOCAL TREE } \\ \square \text { SHRUB } & \square \text { LOCAL SHRUB } \\ \square \text { GRASS } & \square \text { LOCAL GRASS } \\ \square \text { FORBS } & \square \text { LOCAL FORBS } \\ \square \text { WETLAND } & \square \text { LOCAL WETLAND }\end{array}$

RL: www.landarts.com

\section{Norcross}

Delta Landscape Supply GA Inc.

5999 Goshen Springs Rd.

$\square$ TREE

Norcross, GA

30071

PHONE: (770) 448-0805

$\begin{array}{ll}\nabla \text { TREE } & \square \text { LOCAL TREE } \\ \nabla \text { SHRUB } & \square \text { LOCAL SHRUB } \\ \nabla \text { GRASS } & \square \text { LOCAL GRASS } \\ \nabla \text { FORBS } & \square \text { LOCAL FORBS } \\ \square \text { WETLAND } & \square \text { LOCAL WETLAND }\end{array}$

$\square$ SHRUB

$\square$ GRASS

$\square$ LOCAL TREE

$\checkmark$ FORBS

$\square$ LOCAL SHRUB

$\square$ WETLAND

$\square$ LOCAL GRASS

$\square$ LOCAL FORBS

URL: http://Www deltalandscape.com 
Lofts Seed, Inc.

2200 Norcross Pkwy, Suite 255"

$\begin{array}{ll}\square \text { TREE } & \square \text { LOCAL TREE } \\ \square \text { SHRUB } & \square \text { LOCAL SHRUB } \\ \nabla \text { GRASS } & \square \text { LOCAL GRASS } \\ \square \text { FORBS } & \square \text { LOCAL FORBS } \\ \square \text { WETLAND } & \square \text { LOCAL WETLAND }\end{array}$

Norcross, GA

30071

PHONE: (770) 448-9932

$\square$ WETLAND $\square$ LOCAL WETLAND

URL: www.turf.com

\section{Ochlocknee}

Southern Roots Tree Nursery

1810 Bold Springs Rd.

Ochlocknee, GA

31733

PHONE: (912) 377-6237

$\begin{array}{ll}\nabla \text { TREE } & \square \text { LOCAL TREE } \\ \nabla \text { SHRUB } & \checkmark \text { LOCAL SHRUB } \\ \square \text { GRASS } & \square \text { LOCAL GRASS } \\ \square \text { FORBS } & \square \text { LOCAL FORBS } \\ \square \text { WETLAND } & \square \text { LOCAL WETLAND }\end{array}$

URL: none currently available

Pelham

Sawan Seeds Inc.

Box 188

Pelham, GA

31779

PHONE: (229) 294-4953

$\begin{array}{ll}\square \text { TREE } & \square \text { LOCAL TREE } \\ \square \text { SHRUB } & \square \text { LOCAL SHRUB } \\ \nabla \text { GRASS } & \square \text { LOCAL GRASS } \\ \nabla \text { FORBS } & \square \text { LOCAL FORBS } \\ \nabla \text { WETLAND } & \square \text { LOCAL WETLAND }\end{array}$

URL: none currently available

\section{Pine Mountain}

Lazy K Nursery

705 Wright Rd.

Pine Mountain, GA

$\nabla$ TREE

$\checkmark$ SHRUB

$\nabla$ LOCAL TREE

31822

PHONE: (706) 663-4991

$\square$ GRASS $\square$ LOCAL GRASS

$\checkmark$ FORBS

LOCAL GRASS

$\square$ WETLAND

$\square$ LOCAL WETLAND

URL: www.lazyknursery.com

Savannah

Pineland Nursery

PO Box 8242

Savannah, GA

31417

PHONE: (912) 232-6769

$\begin{array}{ll}\nabla \text { TREE } & \square \text { LOCAL TREE } \\ \nabla \text { SHRUB } & \square \text { LOCAL SHRUB } \\ \square \text { GRASS } & \square \text { LOCAL GRASS } \\ \square \text { FORBS } & \square \text { LOCAL FORBS } \\ \square \text { WETLAND } & \square \text { LOCAL WETLAND }\end{array}$

URL: none currently available

Shellman

International Paper/ Georgia Super Tree Nursery

Rt 1, Box 0197

Shellman, GA

31786

PHONE: (800) 554-6550

$\begin{array}{ll}\nabla \text { TREE } & \square \text { LOCAL TREE } \\ \square \text { SHRUB } & \square \text { LOCAL SHRUB } \\ \square \text { GRASS } & \square \text { LOCAL GRASS } \\ \square \text { FORBS } & \square \text { LOCAL FORBS } \\ \square \text { WETLAND } & \square \text { LOCAL WETLAND }\end{array}$

URL: none currently available 
Watkinsville

Athens Seed, Lawn, and Garden

54 Greensboro Highway

Watkinsville, GA

30677

PHONE: (706) 769-2712

$\checkmark$ TREE

$\checkmark$ SHRUB

$\checkmark$ GRASS

$\nabla$ FORBS

$\square$ WETLAND $\checkmark$ LOCAL TREE

$\checkmark$ LOCAL SHRUB

$\square$ LOCAL GRASS

$\square$ LOCAL FORBS

$\square$ LOCAL WETLAND

URL: http:/Wwww athensseed.com

\section{Waynesboro}

C.P. Daniels Sons, Inc.

Box 119

Waynesboro, GA

30830

PHONE: (706) 554-2446

$\begin{array}{ll}\square \text { TREE } & \square \text { LOCAL TREE } \\ \square \text { SHRUB } & \square \text { LOCAL SHRUB } \\ \square \text { GRASS } & \square \text { LOCAL GRASS } \\ \square \text { FORBS } & \square \text { LOCAL FORBS } \\ \square \text { WETLAND } & \square \text { LOCAL WETLAND }\end{array}$

URL: http://www burke.net/cpdaniel

West Point

Adams Nursery

1033 Jarrell Hogg Rd

West Point, GA

31833

PHONE: (706) 882-0447

$\begin{array}{ll}\nabla \text { TREE } & \checkmark \text { LOCAL TREE } \\ \nabla \text { SHRUB } & \checkmark \text { LOCAL SHRUB } \\ \nabla \text { GRASS } & \nabla \text { LOCAL GRASS } \\ \nabla \text { FORBS } & \checkmark \text { LOCAL FORBS } \\ \nabla \text { WETLAND } & \checkmark \text { LOCAL WETLAND }\end{array}$

URL: http:/Wwww.wegrowit.com/adams.catalogue.htm

Marshall Adams

1033 Jarrell Hogg Rd

$\nabla$ TREE

$\checkmark$ SHRUB

$\nabla$ LOCAL TREE

West Point, GA

31833

PHONE: (678) 867-8601

$\square$ GRASS

$\square$ FORBS

$\checkmark$ LOCAL SHRUB

$\square$ LOCAL GRASS

$\square$ LOCAL FORBS

$\square$ WETLAND

$\square$ LOCAL WETLAND

URL: none currently available

Winterville

Thyme After Thyme, Inc.

550 Athens Road

Winterville, GA

30683

PHONE: (706) 742-7149

$\begin{array}{ll}\square \text { TREE } & \square \text { LOCAL TREE } \\ \square \text { SHRUB } & \square \text { LOCAL SHRUB } \\ \nabla \text { GRASS } & \square \text { LOCAL GRASS } \\ \square \text { FORBS } & \square \text { LOCAL FORBS } \\ \square \text { WETLAND } & \square \text { LOCAL WETLAND }\end{array}$

URL: none currently available

\section{Hawaii}

Haiku

Hoolawa Farms

P.O. Box 731

Haiku, HI

9678-0731

PHONE: (808) 572-4835

$\begin{array}{ll}\square \text { TREE } & \square \text { LOCAL TREE } \\ \square \text { SHRUB } & \square \text { LOCAL SHRUB } \\ \square \text { GRASS } & \square \text { LOCAL GRASS } \\ \square \text { FORBS } & \square \text { LOCAL FORBS } \\ \square \text { WETLAND } & \square \text { LOCAL WETLAND }\end{array}$

URL: none currently available 
Hoolehua

Plant Materials Center

4101 Maunaloa Highway

Hoolehua, HI

96729

PHONE: (808) 567-6885

$\begin{array}{ll}\square \text { TREE } & \square \text { LOCAL TREE } \\ \square \text { SHRUB } & \square \text { LOCAL SHRUB } \\ \square \text { GRASS } & \square \text { LOCAL GRASS } \\ \square \text { FORBS } & \square \text { LOCAL FORBS } \\ \square \text { WETLAND } & \square \text { LOCAL WETLAND }\end{array}$

URL: http://plant-materials.nrcs.usda.gov/hipmc/

Kailua-Kona

Sunrise Nursery

73 - 5582 Olowalu St

$\nabla$ TREE $\quad \nabla$ LOCAL TREE

Kailua-Kona, HI

96740

$\checkmark$ SHRUB

$\checkmark$ LOCAL SHRUB

PHONE: (808) 329-7593

$\square$ GRASS $\square$ LOCAL GRASS

$\checkmark$ FORBS $\quad \nabla$ LOCAL FORBS

$\square$ WETLAND $\square$ LOCAL WETLAND

URL: none currently available

Kapa'au

Alkane Nursery

P.O. Box 981

Kapa'au, $\mathrm{HI}$

96755

PHONE: (808) 889-5906

$\begin{array}{ll}\nabla \text { TREE } & \square \text { LOCAL TREE } \\ \nabla \text { SHRUB } & \nabla \text { LOCAL SHRUB } \\ \square \text { GRASS } & \square \text { LOCAL GRASS } \\ \square \text { FORBS } & \square \text { LOCAL FORBS } \\ \square \text { WETLAND } & \square \text { LOCAL WETLAND }\end{array}$

URL: none currently available

\section{lowa}

Adel

Cypripediums Haven

2291 - 280th St

Adel, IA

50003

PHONE: (515) 993-4841

$\begin{array}{ll}\square \text { SHRUB } & \square \text { LOCAL TREE } \\ \square \text { GRASS } & \square \text { LOCAL SHRUB } \\ \square \text { FORBS } & \square \text { LOCAL GRASS } \\ \nabla \text { WOCALAND } & \square \text { LOCAL WERBS } \\ \end{array}$

URL: http://www orchidmall.com/cyp.haven/

Ames

Country Landscape Nursery

56985 Lincoln Highway

Ames, IA

50010

PHONE: (515) 232-6864

$\begin{array}{ll}\nabla \text { TREE } & \square \text { LOCAL TREE } \\ \nabla \text { SHRUB } & \square \text { LOCAL SHRUB } \\ \square \text { GRASS } & \square \text { LOCAL GRASS } \\ \square \text { FORBS } & \square \text { LOCAL FORBS } \\ \square \text { WETLAND } & \square \text { LOCAL WETLAND }\end{array}$

URL: www.countrylandscapes.com

\section{Ankeny}

Des Moines Forage \& Turf Seed

2302 Southeast Creekview Drive

Ankeny, IA

50021

PHONE: (515) 965-4425

$\begin{array}{ll}\square \text { TREE } & \square \text { LOCAL TREE } \\ \square \text { SHRUB } & \square \text { LOCAL SHRUB } \\ \nabla \text { GRASS } & \square \text { LOCAL GRASS } \\ \square \text { FORBS } & \square \text { LOCAL FORBS } \\ \square \text { WETLAND } & \square \text { LOCAL WETLAND }\end{array}$

URL: www.dfseed.com 
Bloomfield

Golden Furrow

RR 6 \#62

Bloomfield, IA

52537

PHONE: (641) 664-1045

$\begin{array}{ll}\square \text { TREE } & \square \text { LOCAL TREE } \\ \square \text { SHRUB } & \square \text { LOCAL SHRUB } \\ \square \text { GRASS } & \square \text { LOCAL GRASS } \\ \square \text { FORBS } & \square \text { LOCAL FORBS } \\ \square \text { WETLAND } & \square \text { LOCAL WETLAND }\end{array}$

URL: none currently available

Carroll

Triple A Seeds, Inc.

1626 Burgess Ave.

Carroll, IA

51401

PHONE: (712) 792-4172

$\begin{array}{ll}\square \text { TREE } & \square \text { LOCAL TREE } \\ \square \text { SHRUB } & \square \text { LOCAL SHRUB } \\ \square \text { GRASS } & \square \text { LOCAL GRASS } \\ \square \text { FORBS } & \square \text { LOCAL FORBS } \\ \square \text { WETLAND } & \square \text { LOCAL WETLAND }\end{array}$

URL: none currently available

\section{Cascade}

Cascade Forestry Service, Inc.

$\begin{array}{lll}21995 \text { Fillmore Rd. } & \nabla \text { TREE } & \square \text { LOCAL TREE } \\ \text { Cascade, IA } & \nabla \text { SHRUB } & \square \text { LOCAL SHRUB } \\ 52033 & \square \text { GRASS } & \square \text { LOCAL GRASS } \\ \text { PHONE: (563) 852-3042 } & \square \text { FORBS } & \square \text { LOCAL FORBS } \\ & \square \text { WETLAND } & \square \text { LOCAL WETLAND }\end{array}$

URL: http:/Wwww.cascadeforestry.com/index.htm

\section{Charles City}

Sherman Nursery $\mathrm{Co}$.

P.O. Box 579

$\square$ TREE $\quad \square$ LOCAL TREE

Charles City, IA

50616

PHONE: (641) 228-1124

$\checkmark$ SHRUB

$\square$ LOCAL SHRUB

$\square$ GRASS

$\square$ LOCAL GRASS

$\square$ FORBS

$\square$ LOCAL FORBS

$\square$ WETLAND $\square$ LOCAL WETLAND

URL: www.shermannursery.com

\section{Davenport}

Freeman Tree Farm \& Nursery

20599 Utica Ridge Rd

Davenport, IA

52807

PHONE: (319) 359-3324

$\begin{array}{ll}\nabla \text { TREE } & \square \text { LOCAL TREE } \\ \nabla \text { SHRUB } & \square \text { LOCAL SHRUB } \\ \nabla \text { GRASS } & \square \text { LOCAL GRASS } \\ \nabla \text { FORBS } & \square \text { LOCAL FORBS } \\ \nabla \text { WETLAND } & \square \text { LOCAL WETLAND }\end{array}$

URL: none currently available

\section{Des Moines}

lowa DNR Bureau of Forestry

Wallace State Office Building

Des Moines, IA

50319-003

PHONE: (515) 242-5966

$\begin{array}{ll}\nabla \text { TREE } & \square \text { LOCAL TREE } \\ \nabla \text { SHRUB } & \square \text { LOCAL SHRUB } \\ \square \text { GRASS } & \square \text { LOCAL GRASS } \\ \square \text { FORBS } & \square \text { LOCAL FORBS } \\ \square \text { WETLAND } & \square \text { LOCAL WETLAND }\end{array}$

URL: http://www. iowadhr.com/forestry/nursery.htm| 
Glenwood

McGinnis Tree and Seed Co

309 E Florence

Glenwood, IA

51534

PHONE: (712) 527-4308

$\begin{array}{ll}\nabla \text { TREE } & \square \text { LOCAL TREE } \\ \nabla \text { SHRUB } & \square \text { LOCAL SHRUB } \\ \nabla \text { GRASS } & \square \text { LOCAL GRASS } \\ \nabla \text { FORBS } & \square \text { LOCAL FORBS } \\ \square \text { WETLAND } & \square \text { LOCAL WETLAND }\end{array}$

URL: none currently available

Hamburg

Sjulin Nurseries

P.O. Box 205

Hamburg, IA

51640

PHONE: (712) 382-2411

$\begin{array}{ll}\square \text { TREE } & \square \text { LOCAL TREE } \\ \nabla \text { SHRUB } & \square \text { LOCAL SHRUB } \\ \square \text { GRASS } & \square \text { LOCAL GRASS } \\ \square \text { FORBS } & \square \text { LOCAL FORBS } \\ \square \text { WETLAND } & \square \text { LOCAL WETLAND }\end{array}$

URL: none currently available

Hampton

Franklin Grassland Seed Co.

$\begin{array}{lll}\text { 1418A 170th St. } & \square \text { TREE } & \square \text { LOCAL TREE } \\ \text { Hampton, IA } & \square \text { SHRUB } & \square \text { LOCAL SHRUB } \\ 50441 & \square \text { GRASS } & \square \text { LOCAL GRASS } \\ \text { PHONE: (641) 456-4410 } & \square \text { FORBS } & \square \text { LOCAL FORBS } \\ & \square \text { WETLAND } & \square \text { LOCAL WETLAND }\end{array}$

URL: none currently available

Harpers Ferry

lon Exchange

1878 Old Mission Dr.

Harpers Ferry IA

52146

PHONE: (563) 535-7231

$\begin{array}{ll}\square \text { TREE } & \square \text { LOCAL TREE } \\ \square \text { SHRUB } & \square \text { LOCAL SHRUB } \\ \square \text { GRASS } & \square \text { LOCAL GRASS } \\ \square \text { FORBS } & \square \text { LOCAL FORBS } \\ \square \text { WETLAND } & \square \text { LOCAL WETLAND }\end{array}$

URL: http://Www ionxchange.com

Johnston

Heard Gardens, Ltd.

5355 Merle Hay Rd.

Johnston, IA

50131

PHONE: (515) 276-4533

$\begin{array}{ll}\nabla \text { TREE } & \square \text { LOCAL TREE } \\ \nabla \text { SHRUB } & \square \text { LOCAL SHRUB } \\ \nabla \text { GRASS } & \square \text { LOCAL GRASS } \\ \nabla \text { FORBS } & \square \text { LOCAL FORBS } \\ \square \text { WETLAND } & \square \text { LOCAL WETLAND }\end{array}$

URL: www.heardgardens.com

\begin{tabular}{|c|c|c|}
\hline Lucas & & \\
\hline Osenbaugh Seed & & \\
\hline RR 1 Box 44 & $\square$ TREE & $\square$ LOCAL TREE \\
\hline Lucas, IA & $\square$ SHRUB & $\square$ LOCAL SHRUB \\
\hline 50151 & $\checkmark$ GRASS & $\square$ LOCAL GRASS \\
\hline PHONE: (641) 766-6790 & $\nabla$ FORBS & $\square$ LOCAL FORBS \\
\hline & $\bar{\nabla}$ WETLAND & $\square$ LOCAL WETLAND \\
\hline
\end{tabular}

URL: none currently available 
Mount Pleasant

Scotts Quality Seeds

Box 110

Mount Pleasant IA

52641

PHONE: (319) 385-8519

$\begin{array}{ll}\square \text { TREE } & \square \text { LOCAL TREE } \\ \square \text { SHRUB } & \square \text { LOCAL SHRUB } \\ \square \text { GRASS } & \square \text { LOCAL GRASS } \\ \square \text { FORBS } & \square \text { LOCAL FORBS } \\ \square \text { WETLAND } & \square \text { LOCAL WETLAND }\end{array}$

URL: http://scottsqualityseeds.com

Perry

Mark Seed Co.

Box 67

Perry, IA

50220

PHONE: (515) 465-2122

$\begin{array}{ll}\square \text { TREE } & \square \text { LOCAL TREE } \\ \square \text { SHRUB } & \square \text { LOCAL SHRUB } \\ \square \text { GRASS } & \square \text { LOCAL GRASS } \\ \square \text { FORBS } & \square \text { LOCAL FORBS } \\ \square \text { WETLAND } & \square \text { LOCAL WETLAND }\end{array}$

URL: none currently available

Rose Hill

Rose Hill Nursery

2282 Teller Ave

Rose Hill, IA

52586

PHONE: (515) 632-8308

$\begin{array}{ll}\square \text { TREE } & \square \text { LOCAL TREE } \\ \nabla \text { SHRUB } & \checkmark \text { LOCAL SHRUB } \\ \nabla \text { GRASS } & \nabla \text { LOCAL GRASS } \\ \nabla \text { FORBS } & \square \text { LOCAL FORBS } \\ \square \text { WETLAND } & \square \text { LOCAL WETLAND }\end{array}$

URL: none currently available

Runnells

Murphys Walnut Hill Nursery

1925 SE 82nd St

Runnells, IA

50237

PHONE: (515) 262-6037

$\begin{array}{ll}\square \text { TREE } & \square \text { LOCAL TREE } \\ \square \text { SHRUB } & \square \text { LOCAL SHRUB } \\ \square \text { GRASS } & \square \text { LOCAL GRASS } \\ \square \text { FORBS } & \square \text { LOCAL FORBS } \\ \square \text { WETLAND } & \square \text { LOCAL WETLAND }\end{array}$

URL: none currently available

\section{Scotch Grove}

Naylor Seed Co.

600 Naylor Dr.

Scotch Grove, IA

52331

PHONE: (319) 465-3035

$\begin{array}{ll}\square \text { TREE } & \square \text { LOCAL TREE } \\ \square \text { SHRUB } & \square \text { LOCAL SHRUB } \\ \square \text { GRASS } & \square \text { LOCAL GRASS } \\ \square \text { FORBS } & \square \text { LOCAL FORBS } \\ \square \text { WETLAND } & \square \text { LOCAL WETLAND }\end{array}$

URL: none currently available

\section{Shenandoah}

Earl May Seed \& Nursery L.P.

$208 \mathrm{~N}$. Elm

Shenandoah, IA

51603

PHONE: (712) 246-1020

$\begin{array}{ll}\square \text { TREE } & \square \text { LOCAL TREE } \\ \square \text { SHRUB } & \square \text { LOCAL SHRUB } \\ \square \text { GRASS } & \square \text { LOCAL GRASS } \\ \square \text { FORBS } & \square \text { LOCAL FORBS } \\ \square \text { WETLAND } & \square \text { LOCAL WETLAND }\end{array}$

URL: www.earlmay.com 


\section{Henry Fields Seed \& Nursery}

$415 \mathrm{~N}$. Burnette

Shenandoah, IA

51602

PHONE: (515) 354-1495

$\begin{array}{ll}\nabla \text { TREE } & \square \text { LOCAL TREE } \\ \nabla \text { SHRUB } & \square \text { LOCAL SHRUB } \\ \nabla \text { GRASS } & \square \text { LOCAL GRASS } \\ \nabla \text { FORBS } & \square \text { LOCAL FORBS } \\ \square \text { WETLAND } & \square \text { LOCAL WETLAND }\end{array}$

URL: http:/Www henryields.com

Mount Arbor Nurseries

PO Box 129

Shenandoah, IA

51601

PHONE: (712) 246-4250

$\begin{array}{ll}\square \text { TREE } & \square \text { LOCAL TREE } \\ \square \text { SHRUB } & \square \text { LOCAL SHRUB } \\ \square \text { GRASS } & \square \text { LOCAL GRASS } \\ \nabla \text { FORBS } & \square \text { LOCAL FORBS } \\ \square \text { WETLAND } & \square \text { LOCAL WETLAND }\end{array}$

URL: none currently available

\section{Sioux Center}

Greenworld Inc.

309 - 7th St NW

Sioux Center, IA

51250

PHONE: (712) 722-2621

$\begin{array}{ll}\nabla \text { TREE } & \square \text { LOCAL TREE } \\ \nabla \text { SHRUB } & \square \text { LOCAL SHRUB } \\ \nabla \text { GRASS } & \square \text { LOCAL GRASS } \\ \nabla \text { FORBS } & \square \text { LOCAL FORBS } \\ \nabla \text { WETLAND } & \square \text { LOCAL WETLAND }\end{array}$

URL: none currently available

Sioux City

Earthtones Inc.; Landscape Management

PO Box 1076

Sioux City, IA

51102

PHONE: (712) 277-0119

$\begin{array}{ll}\square \text { TREE } & \square \text { LOCAL TREE } \\ \square \text { SHRUB } & \square \text { LOCAL SHRUB } \\ \square \text { GRASS } & \square \text { LOCAL GRASS } \\ \square \text { FORBS } & \square \text { LOCAL FORBS } \\ \square \text { WETLAND } & \square \text { LOCAL WETLAND }\end{array}$

URL: none currently available

\section{St. Anthony}

Carl Kurtz

RFD \#1

St. Anthony, IA

50239

PHONE: (515) 477-8364

$\begin{array}{ll}\square \text { TREE } & \square \text { LOCAL TREE } \\ \square \text { SHRUB } & \square \text { LOCAL SHRUB } \\ \nabla \text { GRASS } & \square \text { LOCAL GRASS } \\ \square \text { FORBS } & \square \text { LOCAL FORBS } \\ \square \text { WETLAND } & \square \text { LOCAL WETLAND }\end{array}$

URL: none currently available

Walnut

Heyne Custom Seed Services

26420510 th St.

Walnut, IA

51577

PHONE: (712) 784-3454

$\begin{array}{ll}\square \text { TREE } & \square \text { LOCAL TREE } \\ \square \text { SHRUB } & \square \text { LOCAL SHRUB } \\ \square \text { GRASS } & \nabla \text { LOCAL GRASS } \\ \square \text { FORBS } & \nabla \text { LOCAL FORBS } \\ \square \text { WETLAND } & \square \text { LOCAL WETLAND }\end{array}$

URL: none currently available 
West Point

Merschman Seeds

103 Avenue D

West Point, IA

52656

PHONE: (319) 837-6111

$\begin{array}{ll}\square \text { TREE } & \square \text { LOCAL TREE } \\ \square \text { SHRUB } & \square \text { LOCAL SHRUB } \\ \square \text { GRASS } & \square \text { LOCAL GRASS } \\ \square \text { FORBS } & \square \text { LOCAL FORBS } \\ \square \text { WETLAND } & \square \text { LOCAL WETLAND }\end{array}$

URL: www.merschmanseeds.com

\section{Winterset}

Allendan Seed Company

1966 - 175th Lane

Winterset, IA

50273

PHONE: (515) 462-4084

$\begin{array}{ll}\square \text { TREE } & \square \text { LOCAL TREE } \\ \square \text { SHRUB } & \square \text { LOCAL SHRUB } \\ \square \text { GRASS } & \square \text { LOCAL GRASS } \\ \nabla \text { FORBS } & \square \text { LOCAL FORBS } \\ \square \text { WETLAND } & \square \text { LOCAL WETLAND }\end{array}$

URL: none currently available

\section{Idaho}

Aberdeen

Plant Materials Center

1691 A South 2700 West

Aberdeen, ID

83210-029

PHONE: (208) 397-4133

$\begin{array}{ll}\square \text { TREE } & \square \text { LOCAL TREE } \\ \square \text { SHRUB } & \square \text { LOCAL SHRUB } \\ \square \text { GRASS } & \square \text { LOCAL GRASS } \\ \square \text { FORBS } & \square \text { LOCAL FORBS } \\ \square \text { WETLAND } & \square \text { LOCAL WETLAND }\end{array}$

URL: http://plant-materials.nrcs.usda.govididpmc/

\section{Blackfoot}

Idaho Grimm Growers

Box 276

$\square$ TREE $\quad \square$ LOCAL TREE

Blackfoot, ID

83221

$\checkmark$ SHRUB

$\checkmark$ LOCAL SHRUB

PHONE: (208) 785-0830

$\checkmark$ GRASS

$\checkmark$ LOCAL GRASS

$\checkmark$ FORBS

$\checkmark$ LOCAL FORBS

$\square$ WETLAND $\square$ LOCAL WETLAND

URL: none currently available

\section{Boise}

L.E.Ford Seeds

2918 Woody Dr

Boise, ID

83703

PHONE: (208) 342-8088

$\begin{array}{ll}\square \text { TREE } & \square \text { LOCAL TREE } \\ \nabla \text { SHRUB } & \nabla \text { LOCAL SHRUB } \\ \square \text { GRASS } & \square \text { LOCAL GRASS } \\ \square \text { FORBS } & \square \text { LOCAL FORBS } \\ \square \text { WETLAND } & \square \text { LOCAL WETLAND }\end{array}$

URL: none currently available

\section{The Green Ranch}

P.O. Box 2597

Boise, ID

83701

PHONE: (208) 392-4403

$\begin{array}{ll}\nabla \text { TREE } & \square \text { LOCAL TREE } \\ \nabla \text { SHRUB } & \square \text { LOCAL SHRUB } \\ \square \text { GRASS } & \square \text { LOCAL GRASS } \\ \square \text { FORBS } & \square \text { LOCAL FORBS } \\ \square \text { WETLAND } & \square \text { LOCAL WETLAND }\end{array}$

URL: none currently available 
Bonners Ferry

Clifty View Nursery

R.R. 1, Box 509

Bonners Ferry, ID

83805

PHONE: (208) 267-7129

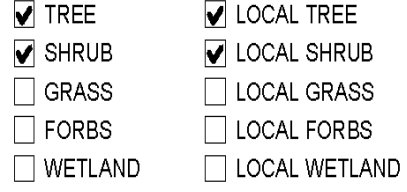

URL: www.cliftwiew.com

Caldwell

Ray Brothers Seed

11140 Chicken Dinner Rd

Caldwell, ID

83605

PHONE: (208) 454-3880

$\begin{array}{ll}\square \text { TREE } & \square \text { LOCAL TREE } \\ \square \text { SHRUB } & \square \text { LOCAL SHRUB } \\ \square \text { GRASS } & \square \text { LOCAL GRASS } \\ \nabla \text { FORBS } & \square \text { LOCAL FORBS } \\ \square \text { WETLAND } & \square \text { LOCAL WETLAND }\end{array}$

URL: none currently available

\section{Gooding}

Lew Pence

1960 U.S. 26

Gooding, ID

83330

PHONE: (208) 934-5302

$\begin{array}{ll}\square \text { TREE } & \square \text { LOCAL TREE } \\ \square \text { SHRUB } & \square \text { LOCAL SHRUB } \\ \square \text { GRASS } & \square \text { LOCAL GRASS } \\ \square \text { FORBS } & \square \text { LOCAL FORBS } \\ \square \text { WETLAND } & \square \text { LOCAL WETLAND }\end{array}$

URL: none currently available

Hailey

Seeds Trust - High Altitude Gardens

4150 Black Oak Drive

Hailey, ID

83333

PHONE: (208) 788-4363

$\begin{array}{ll}\square \text { TREE } & \square \text { LOCAL TREE } \\ \square \text { SHRUB } & \square \text { LOCAL SHRUB } \\ \square \text { GRASS } & \square \text { LOCAL GRASS } \\ \square \text { FORBS } & \square \text { LOCAL FORBS } \\ \square \text { WETLAND } & \square \text { LOCAL WETLAND }\end{array}$

URL: wWw.seedstrust.com

Ketchum

Webb Landscape Inc.

PO Box 744

Ketchum, ID

83340

PHONE: (208) 726-4927

$\begin{array}{ll}\nabla \text { TREE } & \square \text { LOCAL TREE } \\ \square \text { SHRUB } & \square \text { LOCAL SHRUB } \\ \square \text { GRASS } & \square \text { LOCAL GRASS } \\ \square \text { FORBS } & \square \text { LOCAL FORBS } \\ \square \text { WETLAND } & \square \text { LOCAL WETLAND }\end{array}$

URL: www.webbland.com

\section{Lewiston}

Western Forest System, Inc.

1509 Ripon Ave

Lewiston, ID

83501

PHONE: (208) 743-0147

URL: none currently available

$\begin{array}{ll}\square \text { TREE } & \square \text { LOCAL TREE } \\ \square \text { SHRUB } & \square \text { LOCAL SHRUB } \\ \square \text { GRASS } & \square \text { LOCAL GRASS } \\ \square \text { FORBS } & \square \text { LOCAL FORBS } \\ \square \text { WETLAND } & \square \text { LOCAL WETLAND }\end{array}$


McCall

Buffalo-berry Farm

5 I East Lake Fork Rd.

McCall, ID

83638

$\checkmark$ LOCAL TREE

$\checkmark$ SHRUB $\quad \nabla$ LOCAL SHRUB

PHONE: (208) 634-3062

$\nabla$ GRASS $\square$ LOCAL GRASS

$\nabla$ FORBS $\quad \nabla$ LOCAL FORBS

$\square$ WETLAND $\square$ LOCAL WETLAND

URL: none currently available

Melba

Express Farms

HC 79, Box 106C"

Melba, ID

83641

$\square$ TREE

$\square$ SHRUB

$\square$ LOCAL TREE

$\square$ GRASS

$\square$ LOCAL SHRUB

PHONE: (208) 896-4229

$\square$ FORBS

$\square$ LOCAL GRASS

$\checkmark$ WETLAND

$\square$ LOCAL FORBS

$\checkmark$ LOCAL WETLAND

URL: none currently available

Meridan

Jayker Wholesale Nursery

4042 W. Chinden

Meridan, ID

83642

PHONE: (208) 887-1790

$\begin{array}{ll}\square \text { TREE } & \square \text { LOCAL TREE } \\ \nabla \text { SHRUB } & \nabla \text { LOCAL SHRUB } \\ \nabla \text { GRASS } & \nabla \text { LOCAL GRASS } \\ \square \text { FORBS } & \nabla \text { LOCAL FORBS } \\ \square \text { WETLAND } & \square \text { LOCAL WETLAND }\end{array}$

URL: none currently available

\section{Middleton}

Purple Sage Farms, Inc.

11741 Bullock Lane

Middleton, ID

83644

PHONE: (208) 585-6140

$\begin{array}{ll}\square \text { TREE } & \square \text { LOCAL TREE } \\ \square \text { SHRUB } & \square \text { LOCAL SHRUB } \\ \square \text { GRASS } & \square \text { LOCAL GRASS } \\ \square \text { FORBS } & \square \text { LOCAL FORBS } \\ \square \text { WETLAND } & \square \text { LOCAL WETLAND }\end{array}$

URL: none currently available

Moscow

Northplan / Mt. Seed

PO Box 9107

Moscow, ID

83843

PHONE: (208) 882-8040

$\begin{array}{ll}\square \text { TREE } & \square \text { LOCAL TREE } \\ \square \text { SHRUB } & \square \text { LOCAL SHRUB } \\ \square \text { GRASS } & \square \text { LOCAL GRASS } \\ \square \text { FORBS } & \square \text { LOCAL FORBS } \\ \square \text { WETLAND } & \square \text { LOCAL WETLAND }\end{array}$

URL: none currently available

Univ. of Idaho Forest Research Nursery

PO Box 441137

Moscow, ID

83844

PHONE: (208) 885-3888

$\begin{array}{ll}\nabla \text { TREE } & \checkmark \text { LOCAL TREE } \\ \nabla \text { SHRUB } & \square \text { LOCAL SHRUB } \\ \square \text { GRASS } & \square \text { LOCAL GRASS } \\ \square \text { FORBS } & \square \text { LOCAL FORBS } \\ \nabla \text { WETLAND } & \square \text { LOCAL WETLAND }\end{array}$

URL: wwW.seedlings.uidaho.com 


\section{Moyie Springs}

Native Seed Foundation

Star Route

Moyie Springs, ID

83845

PHONE: (208) 267-7938

URL: http://nativeseedfoundation.com

Silver Spring Nursery, Inc.

$\begin{array}{lll}\text { HC 62, Box 86 } & \nabla \text { TREE } & \square \text { LOCAL TREE } \\ \text { Moyie Springs, ID } & \nabla \text { SHRUB } & \square \text { LOCAL SHRUB } \\ 83845 & \square \text { GRASS } & \square \text { LOCAL GRASS } \\ \text { PHONE: (208) 267-5753 } & \nabla \text { FORBS } & \square \text { LOCAL FORBS } \\ & \square \text { WETLAND } & \square \text { LOCAL WETLAND }\end{array}$

URL: none currently available

Nampa

Forage Genetics

1917 E. Fargo Ave.

Nampa, ID

83687

PHONE: (208) 466-9218

$\begin{array}{ll}\nabla \text { TREE } & \square \text { LOCAL TREE } \\ \nabla \text { SHRUB } & \checkmark \text { LOCAL SHRUB } \\ \nabla \text { GRASS } & \square \text { LOCAL GRASS } \\ \square \text { FORBS } & \square \text { LOCAL FORBS } \\ \square \text { WETLAND } & \square \text { LOCAL WETLAND }\end{array}$

URL: none currently available

New Albany

Earthly Goods, Ltd.

P.O. Box 614

New Albany, ID

47150

PHONE: (812) 944-2903

$\begin{array}{ll}\square \text { TREE } & \square \text { LOCAL TREE } \\ \square \text { SHRUB } & \square \text { LOCAL SHRUB } \\ \square \text { GRASS } & \square \text { LOCAL GRASS } \\ \square \text { FORBS } & \square \text { LOCAL FORBS } \\ \square \text { WETLAND } & \square \text { LOCAL WETLAND }\end{array}$

URL: www.earthlygoods.com

Orofino

Reggear Tree Farms

1495 Dent Bridge Rd

Orofino, ID

83544

PHONE: (208) 476-7364

$\begin{array}{ll}\square \text { TREE } & \square \text { LOCAL TREE } \\ \square \text { SHRUB } & \square \text { LOCAL SHRUB } \\ \square \text { GRASS } & \square \text { LOCAL GRASS } \\ \square \text { FORBS } & \square \text { LOCAL FORBS } \\ \square \text { WETLAND } & \square \text { LOCAL WETLAND }\end{array}$

URL: none currently available

\section{Post Falls}

Simplot Turf \& Horticulture

W 5300 Riverbend Ave

Post Falls, ID

83854

PHONE: (208) 773-7581

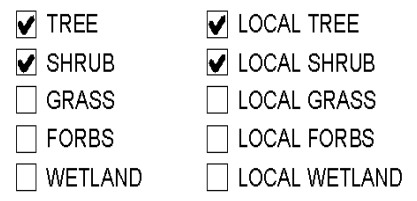

$\square$ WETLAND $\square$ LOCAL WETLAND

URL: www.jacklingolf.com 


\section{Princeton}

Hash Tree Co.

1199 Bearcreek Rd.

Princeton, ID

83857

PHONE: (208) 875-1030

URL: http:/Wwww hashtree.com

Wildlife Habitat Institute

\begin{tabular}{|c|c|c|}
\hline Rt. 1, Box 102-A & $\checkmark$ TREE & $\checkmark$ LOCAL TREE \\
\hline Princeton, ID & $\checkmark$ SHRUB & $\nabla$ LOCAL SHRUB \\
\hline 83857 & $\nabla$ GRASS & $\nabla$ LOCAL GRASS \\
\hline \multirow[t]{2}{*}{ PHONE: (208) 875-1246 } & $\checkmark$ FORBS & $\nabla$ LOCAL FORBS \\
\hline & $\checkmark$ WETLAND & $\checkmark$ LOCAL WETLAND \\
\hline
\end{tabular}

URL: none currently available

$\begin{array}{ll}\square \text { TREE } & \square \text { LOCAL TREE } \\ \square \text { SHRUB } & \square \text { LOCAL SHRUB } \\ \square \text { GRASS } & \square \text { LOCAL GRASS } \\ \square \text { FORBS } & \square \text { LOCAL FORBS } \\ \square \text { WETLAND } & \square \text { LOCAL WETLAND }\end{array}$

Sandpoint

Jenkins \& Son Tree Farm

1014 Elmira Rd

Sandpoint, ID

83864

PHONE: (208) 265-5132

$\begin{array}{ll}\square \text { TREE } & \square \text { LOCAL TREE } \\ \square \text { SHRUB } & \square \text { LOCAL SHRUB } \\ \square \text { GRASS } & \square \text { LOCAL GRASS } \\ \square \text { FORBS } & \square \text { LOCAL FORBS } \\ \square \text { WETLAND } & \square \text { LOCAL WETLAND }\end{array}$

URL: none currently available

\section{Swan Valley}

\section{Cedera Seed}

118 Highway 31

Swan Valley, ID

83449

PHONE: (208) 483-3683

$\begin{array}{ll}\square \text { TREE } & \square \text { LOCAL TREE } \\ \square \text { SHRUB } & \square \text { LOCAL SHRUB } \\ \square \text { GRASS } & \square \text { LOCAL GRASS } \\ \square \text { FORBS } & \square \text { LOCAL FORBS } \\ \square \text { WETLAND } & \square \text { LOCAL WETLAND }\end{array}$

URL: www.cederaseedinc.com

Twin Falls

Globe Feed \& Seed Co.

PO Box 445

Twin Fâlls, ID

83301

PHONE: (208) 733-1373

$\begin{array}{ll}\square \text { TREE } & \square \text { LOCAL TREE } \\ \square \text { SHRUB } & \square \text { LOCAL SHRUB } \\ \square \text { GRASS } & \square \text { LOCAL GRASS } \\ \square \text { FORBS } & \square \text { LOCAL FORBS } \\ \square \text { WETLAND } & \square \text { LOCAL WETLAND }\end{array}$

URL: none currently available

Victor

Trail Creek Nursery, Inc.

18 West 700

Victor, ID

83455

PHONE: (208) 787-2470

$\begin{array}{ll}\nabla \text { TREE } & \square \text { LOCAL TREE } \\ \nabla \text { SHRUB } & \checkmark \text { LOCAL SHRUB } \\ \nabla \text { GRASS } & \checkmark \text { LOCAL GRASS } \\ \square \text { FORBS } & \square \text { LOCAL FORBS } \\ \nabla \text { WETLAND } & \checkmark \text { LOCAL WETLAND }\end{array}$

URL: none currently available 


\section{W. Twin Falls}

Plantasia Cactus Gardens

867 Filer Ave.

W. Twin Falls, ID

83301

PHONE: (208) 734-7959

$\begin{array}{ll}\square \text { TREE } & \square \text { LOCAL TREE } \\ \square \text { SHRUB } & \square \text { LOCAL SHRUB } \\ \square \text { GRASS } & \square \text { LOCAL GRASS } \\ \nabla \text { FORBS } & \square \text { LOCAL FORBS } \\ \square \text { WETLAND } & \square \text { LOCAL WETLAND }\end{array}$

URL: none currently available

\section{Worley}

\section{Lake Creek Seed}

15700 S. Idaho Rd.

Worley, ID

83876

PHONE: (509) 291-6661

$$
\begin{aligned}
& \nabla \text { TREE } \\
& \square \text { SHRUB } \\
& \nabla \text { GRASS } \\
& \nabla \text { FORBS } \\
& \nabla \text { WETLAND }
\end{aligned}
$$

URL: none currently available

\section{IIllinois}

Aroma Park

Kankakee Nursery $\mathrm{C}_{0}$

Box 288

Aroma Park, IL

60910

PHONE: (815) 937-9358

$\begin{array}{ll}\nabla \text { TREE } & \square \text { LOCAL TREE } \\ \nabla \text { SHRUB } & \square \text { LOCAL SHRUB } \\ \nabla \text { GRASS } & \square \text { LOCAL GRASS } \\ \square \text { FORBS } & \square \text { LOCAL FORBS } \\ \square \text { WETLAND } & \square \text { LOCAL WETLAND }\end{array}$

URL: none currently available

\section{Belleville}

Belleville Seed House

3400 S. Illinois St.

Belleville, IL

\begin{tabular}{|c|c|c|}
\hline \multicolumn{3}{|l|}{ Bloomington } \\
\hline \multicolumn{3}{|l|}{ Burgess Seed \& Plant Co. } \\
\hline 905 Four Seasons Rd. & TREE & $\nabla$ LOCAL TREE \\
\hline Bloomington, IL & $\checkmark$ SHRUB & $\nabla$ LOCAL SHRUB \\
\hline 61701 & $\checkmark$ GRASS & $\boldsymbol{v}$ LOCAL GRASS \\
\hline \multirow[t]{2}{*}{ PHONE: (309) 663-9551 } & $\checkmark$ FORBS & $\checkmark$ LOCAL FORBS \\
\hline & $\checkmark$ WETLAND & $\nabla$ LOCAL WETLAND \\
\hline \multicolumn{3}{|c|}{ URL: none currently available } \\
\hline \multicolumn{3}{|l|}{ Owen Nursery } \\
\hline 2300 E. Lincoln St. & $\nabla$ TREE & $\checkmark$ LOCAL TREE \\
\hline Bloomington, IL & $\checkmark$ SHRUB & $\checkmark$ LOCAL SHRUB \\
\hline 61701 & $\square$ GRASS & $\checkmark$ LOCAL GRASS \\
\hline \multirow[t]{2}{*}{ PHONE: (309) 663-9551 } & $\checkmark$ FORBS & $\bar{\nabla}$ LOCAL FORBS \\
\hline & $\checkmark$ WETLAND & $\nabla$ LOCAL WETLAND \\
\hline
\end{tabular}

62220

PHONE: (618) 235-2626

$\begin{array}{ll}\square \text { TREE } & \square \text { LOCAL TREE } \\ \square \text { SHRUB } & \square \text { LOCAL SHRUB } \\ \nabla \text { GRASS } & \square \text { LOCAL GRASS } \\ \nabla \text { FORBS } & \square \text { LOCAL FORBS } \\ \nabla \text { WETLAND } & \square \text { LOCAL WETLAND }\end{array}$

URL: none currently available

URL: http:/Www. owennursery.com/ 
Cerro Gordo

Van Horn Sales

P.O. Box 380

Cerro Gordo, IL

61818

PHONE: (217) 677-2131

$\begin{array}{ll}\square \text { TREE } & \square \text { LOCAL TREE } \\ \square \text { SHRUB } & \square \text { LOCAL SHRUB } \\ \square \text { GRASS } & \square \text { LOCAL GRASS } \\ \square \text { FORBS } & \square \text { LOCAL FORBS } \\ \square \text { WETLAND } & \square \text { LOCAL WETLAND }\end{array}$

URL: none currently available

Cherry Valley

Enders Greenhouse

104 Enders Dr.

Cherry Valley, IL

61016

PHONE: (815) 332-5155

$\begin{array}{ll}\square \text { TREE } & \square \text { LOCAL TREE } \\ \square \text { SHRUB } & \square \text { LOCAL SHRUB } \\ \square \text { GRASS } & \square \text { LOCAL GRASS } \\ \nabla \text { FORBS } & \nabla \text { LOCAL FORBS } \\ \square \text { WETLAND } & \square \text { LOCAL WETLAND }\end{array}$

URL: none currently available

\section{Cobden}

Anna Nursery

PO Box 1

Cobden, IL

62920

PHONE: (618) 893-2124

$\begin{array}{ll}\nabla \text { TREE } & \square \text { LOCAL TREE } \\ \nabla \text { SHRUB } & \square \text { LOCAL SHRUB } \\ \nabla \text { GRASS } & \square \text { LOCAL GRASS } \\ \square \text { FORBS } & \square \text { LOCAL FORBS } \\ \square \text { WETLAND } & \square \text { LOCAL WETLAND }\end{array}$

URL: none currently available

\section{Collinsville}

Nativescapes Nursery

1674 N Bluff Rd

Collinsville, IL

62234

PHONE: (618) 344-8841

$\begin{array}{ll}\nabla \text { TREE } & \square \text { LOCAL TREE } \\ \nabla \text { SHRUB } & \nabla \text { LOCAL SHRUB } \\ \nabla \text { GRASS } & \square \text { LOCAL GRASS } \\ \nabla \text { FORBS } & \nabla \text { LOCAL FORBS } \\ \square \text { WETLAND } & \square \text { LOCAL WETLAND }\end{array}$

URL: none currently available

Crete

Absolutely Wild

25310 S. Stoney Island Ave.

Crete, IL

60417

PHONE: (708) 672-5908

$\begin{array}{ll}\square \text { TREE } & \square \text { LOCAL TREE } \\ \square \text { SHRUB } & \square \text { LOCAL SHRUB } \\ \square \text { GRASS } & \square \text { LOCAL GRASS } \\ \nabla \text { FORBS } & \square \text { LOCAL FORBS } \\ \square \text { WETLAND } & \square \text { LOCAL WETLAND }\end{array}$

URL: none currently available

Gerard \& Green Native Plant Nursery \& Landscape Design

26225 S. Woodlawn Ave

Crete, IL

60417

PHONE: (708) 672-1201

$\begin{array}{ll}\square \text { TREE } & \square \text { LOCAL TREE } \\ \square \text { SHRUB } & \square \text { LOCAL SHRUB } \\ \square \text { GRASS } & \square \text { LOCAL GRASS } \\ \square \text { FORBS } & \square \text { LOCAL FORBS } \\ \square \text { WETLAND } & \square \text { LOCAL WETLAND }\end{array}$

URL: http://WwW gerardandgreene.com/ 
Downers Grove

National Seed Co.

5300 Katrine Ave.

Downers Grove, IL

60515

PHONE: (630) 963-8787

$\begin{array}{ll}\square \text { TREE } & \square \text { LOCAL TREE } \\ \square \text { SHRUB } & \square \text { LOCAL SHRUB } \\ \square \text { GRASS } & \square \text { LOCAL GRASS } \\ \square \text { FORBS } & \square \text { LOCAL FORBS } \\ \square \text { WETLAND } & \square \text { LOCAL WETLAND }\end{array}$

URL: http://simplotpartners.com

\section{Galesburg}

Prairie Restorations

766 Bateman St

Galesburg, IL

61401

PHONE: (309) 343-2608

$\begin{array}{ll}\square \text { TREE } & \square \text { LOCAL TREE } \\ \square \text { SHRUB } & \square \text { LOCAL SHRUB } \\ \square \text { GRASS } & \square \text { LOCAL GRASS } \\ \square \text { FORBS } & \square \text { LOCAL FORBS } \\ \square \text { WETLAND } & \square \text { LOCAL WETLAND }\end{array}$

URL: http://www galesburg.net/ pschramm

Geneseo

C.D. Ford \& Sons, Inc.

16243 Ford Rd.

Geneseo, IL

61254

PHONE: (309) 944-4661

$\begin{array}{ll}\square \text { TREE } & \square \text { LOCAL TREE } \\ \square \text { SHRUB } & \square \text { LOCAL SHRUB } \\ \square \text { GRASS } & \square \text { LOCAL GRASS } \\ \square \text { FORBS } & \square \text { LOCAL FORBS } \\ \square \text { WETLAND } & \square \text { LOCAL WETLAND }\end{array}$

URL: http://Www .cdford.com

Hillsboro

Bluestem Prairie Nursery

RR2

Hillsboro, IL

62049

PHONE: (217) 532-6344

$\begin{array}{ll}\square \text { TREE } & \square \text { LOCAL TREE } \\ \square \text { SHRUB } & \square \text { LOCAL SHRUB } \\ \square \text { GRASS } & \square \text { LOCAL GRASS } \\ \square \text { FORBS } & \square \text { LOCAL FORBS } \\ \square \text { WETLAND } & \square \text { LOCAL WETLAND }\end{array}$

URL: none currently available

\begin{tabular}{|c|c|c|}
\hline \multicolumn{3}{|l|}{ Hinsdale } \\
\hline \multicolumn{3}{|l|}{ Bluff Creek Farm } \\
\hline PO Box 314 & $\nabla$ TREE & $\nabla$ LOCAL TREE \\
\hline Hinsdale, IL & $\square$ SHRUB & $\square$ LOCAL SHRUB \\
\hline 60511 & $\square$ GRASS & $\square$ LOCAL GRASS \\
\hline \multirow[t]{2}{*}{ PHONE: (630) 556-4808 } & $\square$ FORBS & $\square$ LOCAL FORBS \\
\hline & $\nabla$ WETLAND & $\nabla$ LOCAL WETLAND \\
\hline \multicolumn{3}{|c|}{ URL: www.bluffcreektrees.com } \\
\hline \multicolumn{3}{|l|}{ Hinsdale Nurseries, Inc. } \\
\hline 7200 S. Madison & $\checkmark$ TREE & $\checkmark$ LOCAL TREE \\
\hline Hinsdale, IL & $\nabla$ SHRUB & $\checkmark$ LOCAL SHRUB \\
\hline 60521 & $\nabla$ GRASS & $\checkmark$ LOCAL GRASS \\
\hline \multirow[t]{2}{*}{ PHONE: (630) 323-1411 } & $\bar{\nabla}$ FORBS & $\bar{\nabla}$ LOCAL FORBS \\
\hline & $\checkmark$ WETLAND & $\nabla$ LOCAL WETLAND \\
\hline
\end{tabular}

URL: none currently available 


\section{LaFayette}

Lafayette Home Nursery, Inc.

\#1 Nursery Lane

LaFayette, IL

61449

PHONE: (309) 995-3311

URL: http:/Wwwwlafeyettehomenursery.com

$\begin{array}{ll}\nabla \text { TREE } & \nabla \text { LOCAL TREE } \\ \nabla \text { SHRUB } & \checkmark \text { LOCAL SHRUB } \\ \nabla \text { GRASS } & \checkmark \text { LOCAL GRASS } \\ \nabla \text { FORBS } & \square \text { LOCAL FORBS } \\ \nabla \text { WETLAND } & \nabla \text { LOCAL WETLAND }\end{array}$

$\nabla$ WETLAND $\nabla$ LOCAL WETLAND

\section{Lake Zurich}

Hooks Nursery, Inc.

Box 455

Lake Zurich, IL

60047

PHONE: (847) 438-7190

$\begin{array}{ll}\square \text { TREE } & \square \text { LOCAL TREE } \\ \square \text { SHRUB } & \square \text { LOCAL SHRUB } \\ \square \text { GRASS } & \square \text { LOCAL GRASS } \\ \square \text { FORBS } & \square \text { LOCAL FORBS } \\ \square \text { WETLAND } & \square \text { LOCAL WETLAND }\end{array}$

URL: none currently available

\begin{tabular}{|c|c|c|}
\hline \multicolumn{3}{|l|}{ Leland } \\
\hline \multicolumn{3}{|l|}{ Pizzo \& Associates, Inc. } \\
\hline 10729 Pine Road & $\nabla$ TREE & $\checkmark$ LOCAL TREE \\
\hline Leland, IL & $\checkmark$ SHRUB & $\checkmark$ LOCAL SHRUB \\
\hline 60531 & $\nabla$ GRASS & $\nabla$ LOCAL GRASS \\
\hline PHONE: (815) 495-2300 & $\checkmark$ FORBS & $\nabla$ LOCAL FORBS \\
\hline & $\checkmark$ WETLAND & $\checkmark$ LOCAL WETLAND \\
\hline
\end{tabular}

URL: www.pizzo1.com

Monee

Possibility Place Nursery

7548 Manhattan - Monee Rd

Monee, IL

60442

$\nabla$ TREE $\quad \boldsymbol{v}$ LOCAL TREE

$\checkmark$ SHRUB $\quad \nabla$ LOCAL SHRUB

PHONE: (708) 534-3988

$\checkmark$ GRASS $\quad \nabla$ LOCAL GRASS

$\nabla$ FORBS $\quad \nabla$ LOCAL FORBS

$\square$ WETLAND $\square$ LOCAL WETLAND

URL: none currently available

\section{North Litchfield}

H.E. Nursery

1200 Old Rt. 66

North Litchfield, IL

61056

PHONE: (217) 324-6191

$\begin{array}{ll}\nabla \text { TREE } & \nabla \text { LOCAL TREE } \\ \square \text { SHRUB } & \square \text { LOCAL SHRUB } \\ \nabla \text { GRASS } & \square \text { LOCAL GRASS } \\ \nabla \text { FORBS } & \square \text { LOCAL FORBS } \\ \nabla \text { WETLAND } & \nabla \text { LOCAL WETLAND }\end{array}$

URL: none currently available

\section{Northfield}

Hibbard Road Gardens

62 Hibbard Rd

Northfield, IL

60093

PHONE: (847) 446-5525

$\begin{array}{ll}\square \text { TREE } & \square \text { LOCAL TREE } \\ \nabla \text { SHRUB } & \square \text { LOCAL SHRUB } \\ \nabla \text { GRASS } & \square \text { LOCAL GRASS } \\ \nabla \text { FORBS } & \square \text { LOCAL FORBS } \\ \square \text { WETLAND } & \square \text { LOCAL WETLAND }\end{array}$

URL: none currently available 
Ohio

Sand Prairie Farms, Inc.

185 Rogers Rd

Ohio, IL

61349

PHONE: (815) 376-6641

$\begin{array}{ll}\square \text { TREE } & \square \text { LOCAL TREE } \\ \square \text { SHRUB } & \square \text { LOCAL SHRUB } \\ \square \text { GRASS } & \square \text { LOCAL GRASS } \\ \square \text { FORBS } & \square \text { LOCAL FORBS } \\ \square \text { WETLAND } & \square \text { LOCAL WETLAND }\end{array}$

URL: none currently available

Pekin

Sommer Bros. Seed Co

Box 248

Pekin, IL

61555

PHONE: (309) 346-2127

$\begin{array}{ll}\square \text { TREE } & \square \text { LOCAL TREE } \\ \square \text { SHRUB } & \square \text { LOCAL SHRUB } \\ \square \text { GRASS } & \square \text { LOCAL GRASS } \\ \nabla \text { FORBS } & \square \text { LOCAL FORBS } \\ \square \text { WETLAND } & \square \text { LOCAL WETLAND }\end{array}$

URL: http://goldenharvestseeds.com

Peoria

D.A. Hoerr \& Sons Inc.

8020 N Shadetree Dr.

Peoria, IL

61615

PHONE: (309) 691-4561

$\begin{array}{ll}\nabla \text { TREE } & \square \text { LOCAL TREE } \\ \nabla \text { SHRUB } & \square \text { LOCAL SHRUB } \\ \nabla \text { GRASS } & \square \text { LOCAL GRASS } \\ \nabla \text { FORBS } & \square \text { LOCAL FORBS } \\ \nabla \text { WETLAND } & \square \text { LOCAL WETLAND }\end{array}$

URL: www hoernursery.com

\section{Pleasant Plains}

Stone Seed Farms

5965 State Route 97

Pleasant Plains, IL

62677

PHONE: (217) 546-8006

$\begin{array}{ll}\square \text { TREE } & \square \text { LOCAL TREE } \\ \square \text { SHRUB } & \square \text { LOCAL SHRUB } \\ \square \text { GRASS } & \square \text { LOCAL GRASS } \\ \square \text { FORBS } & \square \text { LOCAL FORBS } \\ \square \text { WETLAND } & \square \text { LOCAL WETLAND }\end{array}$

URL: hltp://Nww. stoneseed.com

Pontiac

Wilken Seed Grains, Inc.

P.O. Box 770

Pontiac, IL

61764

PHONE: (815) 844-3458

$\begin{array}{ll}\square \text { TREE } & \square \text { LOCAL TREE } \\ \square \text { SHRUB } & \square \text { LOCAL SHRUB } \\ \square \text { GRASS } & \square \text { LOCAL GRASS } \\ \square \text { FORBS } & \square \text { LOCAL FORBS } \\ \square \text { WETLAND } & \square \text { LOCAL WETLAND }\end{array}$

URL: http:/NWw'wilkenseeds.com

\begin{tabular}{|c|c|c|}
\hline Quincy & & \\
\hline Keller \& Sons & & \\
\hline Box 490 & $\square$ TREE & $\square$ LOCAL TREE \\
\hline Quincy, IL & $\square$ SHRUB & $\square$ LOCAL SHRUB \\
\hline 62301 & $\nabla$ GRASS & $\square$ LOCAL GRASS \\
\hline PHONE: (217) 228-6719 & $\square$ FORBS & $\square$ LOCAL FORBS \\
\hline & $\square$ WETLAND & $\square$ LOCAL WETLAND \\
\hline
\end{tabular}

URL: none currently available 
Rochelle

Country Road Greenhouses, Inc.

19561 E Twombly

Rochelle, IL

61068

$\square$ TOCAL TREE

PHONE: (815) 384-3311

$\checkmark$ SHRUB $\quad \nabla$ LOCAL SHRUB

$\nabla$ GRASS $\quad \nabla$ LOCAL GRASS

$\nabla$ FORBS $\quad \nabla$ LOCAL FORBS

$\nabla$ WETLAND $\square$ LOCAL WETLAND

URL: www.prairieplugs.com

\section{Round Lake}

Synnestvedt Wholesale Nursery $\mathrm{Co}_{0}$

24550 W. Hwy. 120

$\nabla$ TREE $\quad \nabla$ LOCAL TREE

Round Lake, IL

$\checkmark$ SHRUB

$\checkmark$ LOCAL SHRUB

60073

$\square$ GRASS

$\square$ LOCAL GRASS

PHONE: (847) 546-4834

$\begin{array}{ll}\square \text { FORBS } & \square \text { LOCAL FORBS } \\ \square \text { WETLAND } & \square \text { LOCAL WETLAND }\end{array}$

URL: none currently available

Rushville

Boehms Garden Center

RR 4, Box 51

Rushville, IL

62681

PHONE: (217) 322-6644

$\begin{array}{ll}\nabla \text { TREE } & \square \text { LOCAL TREE } \\ \nabla \text { SHRUB } & \square \text { LOCAL SHRUB } \\ \nabla \text { GRASS } & \square \text { LOCAL GRASS } \\ \nabla \text { FORBS } & \square \text { LOCAL FORBS } \\ \square \text { WETLAND } & \square \text { LOCAL WETLAND }\end{array}$

URL: none currently available

\section{St. Charles}

The Natural Garden

38 W. 443 rd, Hwy. 64

St. Charles, IL

60175

PHONE: (630) 584-0150

$\begin{array}{ll}\square \text { TREE } & \square \text { LOCAL TREE } \\ \square \text { SHRUB } & \square \text { LOCAL SHRUB } \\ \square \text { GRASS } & \square \text { LOCAL GRASS } \\ \square \text { FORBS } & \square \text { LOCAL FORBS } \\ \square \text { WETLAND } & \square \text { LOCAL WETLAND }\end{array}$

URL: http://www thenaturalgardeninc.com

Tampico

Genesis Nursery

23200 Hurd Rd.

Tampico, IL

61283

PHONE: (815) 438-2220

$\begin{array}{ll}\square \text { TREE } & \square \text { LOCAL TREE } \\ \square \text { SHRUB } & \square \text { LOCAL SHRUB } \\ \nabla \text { GRASS } & \square \text { LOCAL GRASS } \\ \nabla \text { FORBS } & \square \text { LOCAL FORBS } \\ \nabla \text { WETLAND } & \nabla \text { LOCAL WETLAND }\end{array}$

URL: none currently available

\section{Topeka}

IL Dept. Nat. Res. - Mason State Nursery 17855 N CR $2400 \mathrm{E}$

Topeka, IL

61567

$\nabla$ TREE $\quad \nabla$ LOCAL TREE

$\checkmark$ SHRUB

$\checkmark$ LOCAL SHRUB

PHONE: (309) 535-2185

$\checkmark$ GRASS

$\checkmark$ LOCAL GRASS

$\checkmark$ FORBS

$\checkmark$ LOCAL FORBS

$\checkmark$ WETLAND

$\checkmark$ LOCAL WETLAND

URL: www.dnr.state.il.us 
Tremont

Lees Garden

P.O. Box 5

Tremont, IL

61568

PHONE: (309) 925-5262

$\begin{array}{ll}\square \text { TREE } & \square \text { LOCAL TREE } \\ \square \text { SHRUB } & \square \text { LOCAL SHRUB } \\ \square \text { GRASS } & \square \text { LOCAL GRASS } \\ \square \text { FORBS } & \square \text { LOCAL FORBS } \\ \square \text { WETLAND } & \square \text { LOCAL WETLAND }\end{array}$

URL: none currently available

Urbana

Wandells Nursery, Inc

1898 County Rd. $1700 \mathrm{~N}$.

Urbana, IL

61801

PHONE: (217) 469-2205

$\begin{array}{ll}\square \text { TREE } & \square \text { LOCAL TREE } \\ \square \text { SHRUB } & \square \text { LOCAL SHRUB } \\ \square \text { GRASS } & \square \text { LOCAL GRASS } \\ \square \text { FORBS } & \square \text { LOCAL FORBS } \\ \square \text { WETLAND } & \square \text { LOCAL WETLAND }\end{array}$

URL: none currently available

\section{Woodstock}

Blazing Star, Inc.

2107 Edge Wood Dr

Woodstock, IL

60098

PHONE: (815) 338-4716

URL: www.blazing-star.com

\section{Prairie Haven Ltd.}

17302 US Rt 14

Woodstock, IL

60098

PHONE: (813) 334-0300

$\begin{array}{ll}\square \text { TREE } & \square \text { LOCAL TREE } \\ \square \text { SHRUB } & \square \text { LOCAL SHRUB } \\ \nabla \text { GRASS } & \square \text { LOCAL GRASS } \\ \nabla \text { FORBS } & \square \text { LOCAL FORBS } \\ \square \text { WETLAND } & \square \text { LOCAL WETLAND }\end{array}$

URL: none currently available

\section{$\checkmark$ \& J Seed Farms, Inc.}

P O Box 82

Woodstock, IL

60098

PHONE: (815) 338-4029

$\begin{array}{ll}\square \text { TREE } & \square \text { LOCAL TREE } \\ \square \text { SHRUB } & \square \text { LOCAL SHRUB } \\ \square \text { GRASS } & \square \text { LOCAL GRASS } \\ \square \text { FORBS } & \square \text { LOCAL FORBS } \\ \square \text { WETLAND } & \square \text { LOCAL WETLAND }\end{array}$

$\begin{array}{ll}\square \text { TREE } & \square \text { LOCAL TREE } \\ \square \text { SHRUB } & \square \text { LOCAL SHRUB } \\ \square \text { GRASS } & \square \text { LOCAL GRASS } \\ \nabla \text { FORBS } & \square \text { LOCAL FORBS } \\ \square \text { WETLAND } & \square \text { LOCAL WETLAND }\end{array}$

URL: none currently available

\section{Wyoming}

Purple Prarie Farm

RR2, Box 176"

Wyoming, IL

61491

PHONE: (309) 286-7356

$\begin{array}{ll}\square \text { TREE } & \square \text { LOCAL TREE } \\ \square \text { SHRUB } & \square \text { LOCAL SHRUB } \\ \nabla \text { GRASS } & \nabla \text { LOCAL GRASS } \\ \square \text { FORBS } & \boldsymbol{\nabla} \text { LOCAL FORBS } \\ \square \text { WETLAND } & \square \text { LOCAL WETLAND }\end{array}$

URL: none currently available 
Zion

Arthur Weiler Inc. Nursery

12247 W. Russell Rd.

Zion, IL

$\checkmark$ TREE

$\checkmark$ SHRUB

60099

PHONE: (847) 746-2393

$\square$ GRASS

$\square$ FORBS

$\checkmark$ LOCAL TREE

$\square$ WETLAND

$\checkmark$ LOCAL SHRUB

$\square$ LOCAL GRASS

$\square$ LOCAL FORBS

URL: www.weilernursery.com

\section{Indiana}

Auburn

Hoham Smith \& Co.

P.O. Box 710

Auburn, IN

46706

PHONE: (260) 925-3400

$\begin{array}{ll}\nabla \text { TREE } & \square \text { LOCAL TREE } \\ \nabla \text { SHRUB } & \square \text { LOCAL SHRUB } \\ \nabla \text { GRASS } & \square \text { LOCAL GRASS } \\ \nabla \text { FORBS } & \square \text { LOCAL FORBS } \\ \square \text { WETLAND } & \square \text { LOCAL WETLAND }\end{array}$

URL: none currently available

\section{Charles City}

Smith Nursery Co.

PO Box 515

Charles City, IN

50616

PHONE: (515) 228-3239

$\begin{array}{ll}\nabla \text { TREE } & \square \text { LOCAL TREE } \\ \nabla \text { SHRUB } & \square \text { LOCAL SHRUB } \\ \square \text { GRASS } & \square \text { LOCAL GRASS } \\ \square \text { FORBS } & \square \text { LOCAL FORBS } \\ \square \text { WETLAND } & \square \text { LOCAL WETLAND }\end{array}$

URL: none currently available

\section{Depauw}

Munchkin Nursery \& Garden

323 Woodside Drive NW

Depauw, IN

47115

PHONE: (812) 633-4858

$\begin{array}{ll}\square \text { TREE } & \square \text { LOCAL TREE } \\ \square \text { SHRUB } & \square \text { LOCAL SHRUB } \\ \square \text { GRASS } & \square \text { LOCAL GRASS } \\ \square \text { FORBS } & \square \text { LOCAL FORBS } \\ \square \text { WETLAND } & \square \text { LOCAL WETLAND }\end{array}$

URL: www.munchkinnursery.com

Hamlet

Hensler Nursery, Inc.

5715 N $750 \mathrm{E}$

Hamlet, IN

46532

PHONE: (219) 867-4192

$\begin{array}{ll}\nabla \text { TREE } & \square \text { LOCAL TREE } \\ \nabla \text { SHRUB } & \square \text { LOCAL SHRUB } \\ \square \text { GRASS } & \square \text { LOCAL GRASS } \\ \square \text { FORBS } & \square \text { LOCAL FORBS } \\ \square \text { WETLAND } & \square \text { LOCAL WETLAND }\end{array}$

URL: none currently available

\section{Haubstadt}

Tenbarge Seed Co., Inc.

$$
\text { Box } 187
$$

Haubstadt, IN

47639

PHONE: (812) 768-6157

$\begin{array}{ll}\square \text { TREE } & \square \text { LOCAL TREE } \\ \square \text { SHRUB } & \square \text { LOCAL SHRUB } \\ \square \text { GRASS } & \square \text { LOCAL GRASS } \\ \square \text { FORBS } & \square \text { LOCAL FORBS } \\ \square \text { WETLAND } & \square \text { LOCAL WETLAND }\end{array}$

URL: www.tenbargeseeds.com 
Lizton

Berg-Warner Nursery, Inc.

PO Box 259

Lizton, IN

46149

PHONE: (317) 994-5487

$\begin{array}{ll}\checkmark \text { TREE } & \checkmark \text { LOCAL TREE } \\ \square \text { SHRUB } & \square \text { LOCAL SHRUB } \\ \square \text { GRASS } & \square \text { LOCAL GRASS } \\ \square \text { FORBS } & \square \text { LOCAL FORBS } \\ \square \text { WETLAND } & \square \text { LOCAL WETLAND }\end{array}$

URL: www.berg-warner.com

\section{Malkerton}

J.F. New \& Associates, Inc. 708 Roosevelt Rd.

$\nabla$ TREE $\quad \nabla$ LOCAL TREE

Malkerton, IN

46574

$\checkmark$ SHRUB $\quad \nabla$ LOCAL SHRUB

PHONE: (219) 586-3400

$\checkmark$ GRASS $\quad \nabla$ LOCAL GRASS

$\nabla$ FORBS $\quad \nabla$ LOCAL FORBS

$\nabla$ WETLAND $\quad \nabla$ LOCAL WETLAND

URL: www.fnew.com

\section{Medaryville}

Jasper-Pulaski Nursery

$\begin{array}{lll}15508 \text { West } 700 \text { North } & \square \text { TREE } & \square \text { LOCAL TREE } \\ \text { Medaryville, IN } & \square \text { SHRUB } & \square \text { LOCAL SHRUB } \\ 47957 & \square \text { GRASS } & \square \text { LOCAL GRASS } \\ \text { PHONE: (219) 843-4827 } & \square \text { FORBS } & \square \text { LOCAL FORBS } \\ & \square \text { WETLAND } & \square \text { LOCAL WETLAND }\end{array}$

URL: http://Www state.in.us/dnr/forestry/

Muncie

Spence Restoration Nursery, Inc.

$2220 \mathrm{E}$. Fuson Rd.

$\checkmark$ TREE $\quad \nabla$ LOCAL TREE

Muncie, IN

47308

$\checkmark$ SHRUB

$\nabla$ LOCAL SHRUB

PHONE: (765) 286-7154

$\checkmark$ GRASS $\square$ LOCAL GRASS

$\checkmark$ FORBS $\quad \nabla$ LOCAL FORBS

$\checkmark$ WETLAND $\square$ LOCAL WETLAND

URL: none currently available

\section{Petersburg}

Wyatt Seed Co

$\begin{array}{lll}\text { Box } 218 & \square \text { TREE } & \square \text { LOCAL TREE } \\ \text { Petersburg, IN } & \square \text { SHRUB } & \square \text { LOCAL SHRUB } \\ \text { 47567 } & \square \text { GRASS } & \square \text { LOCAL GRASS } \\ \text { PHONE: (812) 354-8542 } & \square \text { FORBS } & \square \text { LOCAL FORBS } \\ & \square \text { WETLAND } & \square \text { LOCAL WETLAND }\end{array}$

URL: none currently available

\begin{tabular}{|c|c|c|}
\hline Vallonia & & \\
\hline Vallonia Nursery & & \\
\hline 2782 W. County Rd $540 \mathrm{~S}$ & $\checkmark$ TREE & $\checkmark$ LOCAL TREE \\
\hline Vallonia, IN & $\checkmark$ SHRUB & $\checkmark$ LOCAL SHRUB \\
\hline 47281 & $\square$ GRASS & $\square$ LOCAL GRASS \\
\hline PHONE: (812) 358-3621 & $\square$ FORBS & $\square$ LOCAL FORBS \\
\hline & $\square$ WETLAND & $\square$ LOCAL WETLAND \\
\hline
\end{tabular}

URL: http://WwW state.in.us/dnr/forestry/ 
Whiteland

Maschmeyers Nursery, Inc.

3009 E. 500 N. P.O. Box 8

$\nabla$ TREE $\square$ LOCAL TREE

Whiteland, IN

46184

PHONE: (317) 535-7541

$\square$ SHRUB $\square$ LOCAL SHRUB

$\square$ GRASS $\square$ LOCAL GRASS

$\square$ FORBS $\square$ LOCAL FORBS

URL: none currently available

\section{Kansas}

Alta Vista

Double-Bar Pine Seed \& Tree Nursery

9812 Highway K-177

Alta Vista, KS

66834

PHONE: (785) 499-6328

$\square$ WETLAND $\square$ LOCAL WETLAND

URL: www.doublebarpinenursery.com

\section{Cherryvace}

Sunflower Farms

Rt. 2, Box 26AA

Cherryace, KS

67335

PHONE: (620) 336-2066

$\begin{array}{ll}\nabla \text { TREE } & \square \text { LOCAL TREE } \\ \nabla \text { SHRUB } & \square \text { LOCAL SHRUB } \\ \nabla \text { GRASS } & \square \text { LOCAL GRASS } \\ \square \text { FORBS } & \square \text { LOCAL FORBS } \\ \square \text { WETLAND } & \square \text { LOCAL WETLAND }\end{array}$

URL: none currently available

\section{Dodge City}

Casterline West

P.O. Bos 1377

Dodge City, KS

67801

PHONE: (620) 225-4137

$\begin{array}{ll}\square \text { TREE } & \square \text { LOCAL TREE } \\ \square \text { SHRUB } & \square \text { LOCAL SHRUB } \\ \square \text { GRASS } & \square \text { LOCAL GRASS } \\ \square \text { FORBS } & \square \text { LOCAL FORBS } \\ \square \text { WETLAND } & \square \text { LOCAL WETLAND }\end{array}$

$\square$ WETLAND

$\square$ LOCAL WETLAND

URL none currentlyavailable

URL: $\underline{\text { none currently available }}$

Girard

Delange Seed House, Inc.

Box 7, RR2"

Girard, KS

66743

PHONE: (620) 724-6223

$\begin{array}{ll}\square \text { TREE } & \square \text { LOCAL TREE } \\ \square \text { SHRUB } & \square \text { LOCAL SHRUB } \\ \square \text { GRASS } & \square \text { LOCAL GRASS } \\ \square \text { FORBS } & \square \text { LOCAL FORBS } \\ \square \text { WETLAND } & \square \text { LOCAL WETLAND }\end{array}$

$\checkmark$ WETLAND $\square$ LOCAL WETLAND

URL: www.delangeseed.com

\section{Healy}

Sharp Bros. Seed Co.

Box 140

Healy, KS

67850

PHONE: (316) 398-2231

$\begin{array}{ll}\square \text { TREE } & \square \text { LOCAL TREE } \\ \square \text { SHRUB } & \square \text { LOCAL SHRUB } \\ \square \text { GRASS } & \square \text { LOCAL GRASS } \\ \square \text { FORBS } & \square \text { LOCAL FORBS } \\ \square \text { WETLAND } & \square \text { LOCAL WETLAND }\end{array}$

$\begin{array}{ll}\square \text { TREE } & \square \text { LOCAL TREE } \\ \square \text { SHRUB } & \square \text { LOCAL SHRUB } \\ \nabla \text { GRASS } & \square \text { LOCAL GRASS } \\ \square \text { FORBS } & \square \text { LOCAL FORBS } \\ \square \text { WETLAND } & \square \text { LOCAL WETLAND }\end{array}$

URL: www.sharpseed.com 
Manhattan

Kansas Forest Service

2610 Clafin Rd

Manhattan, KS

66502-279

PHONE: (785) 532-3300

URL: http:/Wwww.kansasforests.org/

\section{Plant Materials Center}

3800 S. 20 th St.

Manhattan, KS

66502

PHONE: (785) 539-8761

$\begin{array}{ll}\nabla \text { TREE } & \square \text { LOCAL TREE } \\ \square \text { SHRUB } & \square \text { LOCAL SHRUB } \\ \square \text { GRASS } & \square \text { LOCAL GRASS } \\ \square \text { FORBS } & \square \text { LOCAL FORBS } \\ \square \text { WETLAND } & \square \text { LOCAL WETLAND }\end{array}$

URL: http://plant-materials.nrcs.usda.gov/kspmc/

$\begin{array}{ll}\square \text { TREE } & \square \text { LOCAL TREE } \\ \square \text { SHRUB } & \square \text { LOCAL SHRUB } \\ \square \text { GRASS } & \square \text { LOCAL GRASS } \\ \square \text { FORBS } & \square \text { LOCAL FORBS } \\ \square \text { WETLAND } & \square \text { LOCAL WETLAND }\end{array}$

\section{Lodge}

Glenn Snell

$300 \mathrm{~N}$. Adams

Medicine Lodge, KS

67104

PHONE: (620) 886-5075

$\begin{array}{ll}\square \text { TREE } & \square \text { LOCAL TREE } \\ \square \text { SHRUB } & \square \text { LOCAL SHRUB } \\ \nabla \text { GRASS } & \square \text { LOCAL GRASS } \\ \nabla \text { FORBS } & \square \text { LOCAL FORBS } \\ \square \text { WETLAND } & \square \text { LOCAL WETLAND }\end{array}$

URL: www.luka.com

Quivira Management Inc.

$300 \mathrm{~N}$ Adams

Medicine Lodge, KS

67104

PHONE: (316) 886-5075

$\begin{array}{ll}\square \text { TREE } & \square \text { LOCAL TREE } \\ \square \text { SHRUB } & \square \text { LOCAL SHRUB } \\ \square \text { GRASS } & \square \text { LOCAL GRASS } \\ \square \text { FORBS } & \square \text { LOCAL FORBS } \\ \square \text { WETLAND } & \square \text { LOCAL WETLAND }\end{array}$

URL: www.laka.com

\section{Mound Valley}

Johnson Seed Co.

14038 Grey Rd

Mound Valley, KS

67354

PHONE: (620) 328-2321

$\begin{array}{ll}\square \text { TREE } & \square \text { LOCAL TREE } \\ \square \text { SHRUB } & \square \text { LOCAL SHRUB } \\ \square \text { GRASS } & \square \text { LOCAL GRASS } \\ \square \text { FORBS } & \square \text { LOCAL FORBS } \\ \square \text { WETLAND } & \square \text { LOCAL WETLAND }\end{array}$

URL: none currently available

Olathe

Tri-Star Seed Co.

15257 S. Keeler St.

Olathe, KS

66062

PHONE: (913) 780-6166

$\begin{array}{ll}\square \text { TREE } & \square \text { LOCAL TREE } \\ \square \text { SHRUB } & \square \text { LOCAL SHRUB } \\ \square \text { GRASS } & \square \text { LOCAL GRASS } \\ \square \text { FORBS } & \square \text { LOCAL FORBS } \\ \square \text { WETLAND } & \square \text { LOCAL WETLAND }\end{array}$

URL: none currently available 
Osborne

Star Seed, Inc.

Box 228

Osborne, KS

67473

$\square$ LOCAL TREE

PHONE: (785) 346-5447

$\square$ SHRUB

$\checkmark$ GRASS

$\square$ LOCAL SHRUB

$\checkmark$ LOCAL GRASS

URL: www.gostarseed.com

\section{Prairie Village}

Bluestem Seed Co.

4045 Somerset Dr.

Prairie Village, KS

66208

PHONE: (913) 642-7106

$\begin{array}{ll}\square \text { TREE } & \square \text { LOCAL TREE } \\ \square \text { SHRUB } & \square \text { LOCAL SHRUB } \\ \square \text { GRASS } & \square \text { LOCAL GRASS } \\ \square \text { FORBS } & \square \text { LOCAL FORBS } \\ \square \text { WETLAND } & \square \text { LOCAL WETLAND }\end{array}$

URL: none currently available

\section{Sedgwick}

Delange Seed, Inc.

610 N. Washington St.

Sedgwick, KS

67135

PHONE: (316) 772-5388

$\begin{array}{ll}\square \text { TREE } & \square \text { LOCAL TREE } \\ \square \text { SHRUB } & \square \text { LOCAL SHRUB } \\ \nabla \text { GRASS } & \square \text { LOCAL GRASS } \\ \square \text { FORBS } & \square \text { LOCAL FORBS } \\ \square \text { WETLAND } & \square \text { LOCAL WETLAND }\end{array}$

URL: www.delangeseed.com

\section{Wichita}

Valley Feed \& Seed

1903 S. Meridian

Wichita, KS

67213

PHONE: (316) 942-2278

$\begin{array}{ll}\square \text { TREE } & \square \text { LOCAL TREE } \\ \square \text { SHRUB } & \square \text { LOCAL SHRUB } \\ \square \text { GRASS } & \square \text { LOCAL GRASS } \\ \square \text { FORBS } & \square \text { LOCAL FORBS } \\ \square \text { WETLAND } & \square \text { LOCAL WETLAND }\end{array}$

URL: none currently available

\section{Kentucky}

Frankfort

Shooting Star Nursery

444 Bates Rd.

Frankfort, KY

40601

PHONE: (502) 223-1679

$\begin{array}{ll}\nabla \text { TREE } & \nabla \text { LOCAL TREE } \\ \nabla \text { SHRUB } & \nabla \text { LOCAL SHRUB } \\ \nabla \text { GRASS } & \nabla \text { LOCAL GRASS } \\ \nabla \text { FORBS } & \nabla \text { LOCAL FORBS } \\ \nabla \text { WETLAND } & \nabla \text { LOCAL WETLAND }\end{array}$

URL: none currently available

\section{Hardinsburg}

Dobbs Seed \& Grain Co

Box 489

Hardinsburg, $\mathrm{KY}$

40143

PHONE: (502) 756-2311

$\begin{array}{ll}\square \text { TREE } & \square \text { LOCAL TREE } \\ \square \text { SHRUB } & \square \text { LOCAL SHRUB } \\ \nabla \text { GRASS } & \square \text { LOCAL GRASS } \\ \square \text { FORBS } & \square \text { LOCAL FORBS } \\ \square \text { WETLAND } & \square \text { LOCAL WETLAND }\end{array}$

URL: none currently available 
Louisville

Dabney Herbs

Box 22061

Louisville, KY

40252

PHONE: (502) 893-5198

URL: www.dabneyherbs.com

Lewis Seed Co.

$130 \mathrm{~N}$. Spring St.

Louisville, KY

40206

PHONE: (502) 587-1241

$\begin{array}{ll}\square \text { TREE } & \square \text { LOCAL TREE } \\ \square \text { SHRUB } & \square \text { LOCAL SHRUB } \\ \square \text { GRASS } & \square \text { LOCAL GRASS } \\ \square \text { FORBS } & \square \text { LOCAL FORBS } \\ \square \text { WETLAND } & \square \text { LOCAL WETLAND }\end{array}$

URL: none currently available

Morehead

Caudill Seed Company

1064 East Main Street

Morehead, KY

40351

PHONE: (606) 780-0848

$\begin{array}{ll}\nabla \text { TREE } & \square \text { LOCAL TREE } \\ \square \text { SHRUB } & \square \text { LOCAL SHRUB } \\ \nabla \text { GRASS } & \square \text { LOCAL GRASS } \\ \square \text { FORBS } & \square \text { LOCAL FORBS } \\ \square \text { WETLAND } & \square \text { LOCAL WETLAND }\end{array}$

$\square$ WETLAND $\square$ LOCAL WETLAND

JRL: www.caudillseed.com

\section{Owenton}

Janes Native Seeds

1860 Kays Branch Rd.

Owenton, KY

40359

PHONE: (502) 484-2044

$\begin{array}{ll}\square \text { TREE } & \square \text { LOCAL TREE } \\ \square \text { SHRUB } & \square \text { LOCAL SHRUB } \\ \square \text { GRASS } & \square \text { LOCAL GRASS } \\ \square \text { FORBS } & \square \text { LOCAL FORBS } \\ \square \text { WETLAND } & \square \text { LOCAL WETLAND }\end{array}$

URL: none currently available

Springfield

Valley Hill Nurseries

4251 Broomfield Rd.

Springfield, KY

40069

PHONE: (606) 336-9017

$\begin{array}{ll}\nabla \text { TREE } & \nabla \text { LOCAL TREE } \\ \nabla \text { SHRUB } & \nabla \text { LOCAL SHRUB } \\ \nabla \text { GRASS } & \checkmark \text { LOCAL GRASS } \\ \nabla \text { FORBS } & \nabla \text { LOCAL FORBS } \\ \nabla \text { WETLAND } & \nabla \text { LOCAL WETLAND }\end{array}$

v WetLand

LOCAL WETLAND

URL: none currently available

West Liberty

Morgan County Nursery

438 Tree Nursery Rd

West Liberty, KY

41472

PHONE: (606) 743-3511

$\begin{array}{ll}\square \text { TREE } & \square \text { LOCAL TREE } \\ \square \text { SHRUB } & \square \text { LOCAL SHRUB } \\ \square \text { GRASS } & \square \text { LOCAL GRASS } \\ \square \text { FORBS } & \square \text { LOCAL FORBS } \\ \square \text { WETLAND } & \square \text { LOCAL WETLAND }\end{array}$

URL: www.forestry.ky.gov

$\begin{array}{ll}\square \text { TREE } & \square \text { LOCAL TREE } \\ \square \text { SHRUB } & \square \text { LOCAL SHRUB } \\ \square \text { GRASS } & \square \text { LOCAL GRASS } \\ \square \text { FORBS } & \square \text { LOCAL FORBS } \\ \square \text { WETLAND } & \square \text { LOCAL WETLAND }\end{array}$




\section{Louisiana}

Abbeville

Coast Plants

2320 Alcide Cir.

Abbeville, LA

70510

$\begin{array}{ll}\square \text { TREE } & \square \text { LOCAL TREE } \\ \square \text { SHRUB } & \square \text { LOCAL SHRUB } \\ \square \text { GRASS } & \square \text { LOCAL GRASS } \\ \square \text { FORBS } & \square \text { LOCAL FORBS } \\ \square \text { WETLAND } & \square \text { LOCAL WETLAND }\end{array}$

PHONE: (337) 898-3098

$\checkmark$ WETLAND

$\checkmark$ LOCAL WETLAND

URL: none currently available

\section{Amite}

Jenkins Farm and Nursery

62188 Dummyline Ro

Amite, LA

70422

PHONE: (504) 748-7746

$\begin{array}{ll}\square \text { TREE } & \square \text { LOCAL TREE } \\ \square \text { SHRUB } & \square \text { LOCAL SHRUB } \\ \square \text { GRASS } & \square \text { LOCAL GRASS } \\ \square \text { FORBS } & \square \text { LOCAL FORBS } \\ \square \text { WETLAND } & \square \text { LOCAL WETLAND }\end{array}$

URL: none currently available

Louisiana Growers

63279 Lowery Rd.

Amite, LA

TREE LOCAL TREE

70422

PHONE: (504) 748-5850

$\nabla$ SHRUB $\quad \nabla$ LOCAL SHRUB

$\square$ GRASS $\square$ LOCAL GRASS

$\checkmark$ FORBS $\square$ LOCAL FORBS

$\nabla$ WETLAND $\nabla$ LOCAL WETLAND

URL: www.louisianagrowers.com

Ball

Duncan Nurseries, Inc.

Box 96

Bâll, LA

71405

PHONE: (318) 640-9772

$\begin{array}{ll}\nabla \text { TREE } & \square \text { LOCAL TREE } \\ \square \text { SHRUB } & \square \text { LOCAL SHRUB } \\ \square \text { GRASS } & \square \text { LOCAL GRASS } \\ \square \text { FORBS } & \square \text { LOCAL FORBS } \\ \square \text { WETLAND } & \square \text { LOCAL WETLAND }\end{array}$

URL: www.duncannurseries.com

Covington

Bills Creek Nursery

82406 Fussell Cemetary Rd.

Covington, LA

70433

PHONE: (985) 892-2327

$\begin{array}{ll}\square \text { TREE } & \square \text { LOCAL TREE } \\ \nabla \text { SHRUB } & \square \text { LOCAL SHRUB } \\ \square \text { GRASS } & \square \text { LOCAL GRASS } \\ \square \text { FORBS } & \square \text { LOCAL FORBS } \\ \square \text { WETLAND } & \square \text { LOCAL WETLAND }\end{array}$

URL: none currently available

\section{Deridder}

Beauregard Nursery

Box 935

Deridder, LA

70634

PHONE: (337) 463-5509

$\begin{array}{ll}\nabla \text { TREE } & \square \text { LOCAL TREE } \\ \square \text { SHRUB } & \square \text { LOCAL SHRUB } \\ \square \text { GRASS } & \square \text { LOCAL GRASS } \\ \square \text { FORBS } & \square \text { LOCAL FORBS } \\ \square \text { WETLAND } & \square \text { LOCAL WETLAND }\end{array}$

URL: www.LDAF.statela.us 
Folsom

\section{Brumfield Nursery}

Box 653

Folsom, LA

70437

PHONE: (985) 796-0687

URL: none currently available

\section{Folsom Nursery, Inc.}

14425 Hwy. $40 \mathrm{E}$

Folsom, LA

70437

PHONE: (985) 796-3488

URL: none currently available

\section{Garretts Nursery}

Box 1116

Folsom, LA

70437

PHONE: (985) 796-3310

URL: none currently available

Windmill Nurseries, Inc.

P.O. Box 400

Folsom, LA

70437

PHONE: (800) 535-1887

URL: none currently available

$\begin{array}{ll}\nabla \text { TREE } & \nabla \text { LOCAL TREE } \\ \nabla \text { SHRUB } & \square \text { LOCAL SHRUB } \\ \square \text { GRASS } & \square \text { LOCAL GRASS } \\ \square \text { FORBS } & \square \text { LOCAL FORBS } \\ \square \text { WETLAND } & \square \text { LOCAL WETLAND }\end{array}$

$\checkmark$ TREE

$\checkmark$ SHRUB

$\square$ GRASS

$\square$ FORBS

$\square$ WETLAND

$\checkmark$ LOCAL TREE

$\checkmark$ LOCAL SHRUB

$\square$ LOCAL GRASS

$\square$ LOCAL FORBS

$\square$ LOCAL WETLAND

$\begin{array}{ll}\nabla \text { TREE } & \square \text { LOCAL TREE } \\ \nabla \text { SHRUB } & \square \text { LOCAL SHRUB } \\ \square \text { GRASS } & \square \text { LOCAL GRASS } \\ \square \text { FORBS } & \square \text { LOCAL FORBS } \\ \nabla \text { WETLAND } & \square \text { LOCAL WETLAND }\end{array}$

$\begin{array}{ll}\nabla \text { TREE } & \square \text { LOCAL TREE } \\ \nabla \text { SHRUB } & \square \text { LOCAL SHRUB } \\ \square \text { GRASS } & \square \text { LOCAL GRASS } \\ \square \text { FORBS } & \square \text { LOCAL FORBS } \\ \square \text { WETLAND } & \square \text { LOCAL WETLAND }\end{array}$

\section{Forest Hill}

Chandlers Nursery

18 Forest Dr. Highway 112

$\square$ TREE

Forest Hill, LA

71430

PHONE: (318) 748-6339

$\checkmark$ SHRUB

$\square$ LOCAL TREE

$\square$ GRASS

$\checkmark$ LOCAL SHRUB

$\square$ FORBS

$\square$ LOCAL GRASS

$\square$ WETLAND

$\square$ LOCAL FORBS

URL: none currently available

Doug Youngs Nursery

Box 39

Forest Hill, LA

71430

PHONE: (318) 748-6071

$\begin{array}{ll}\nabla \text { TREE } & \square \text { LOCAL TREE } \\ \square \text { SHRUB } & \square \text { LOCAL SHRUB } \\ \square \text { GRASS } & \square \text { LOCAL GRASS } \\ \square \text { FORBS } & \square \text { LOCAL FORBS } \\ \nabla \text { WETLAND } & \square \text { LOCAL WETLAND }\end{array}$

URL: www.dougyoungnursery.com

George Johnson Nursery

3848 Highway 112

Forest Hill, LA

71430

PHONE: (318) 748-6715

$\begin{array}{ll}\square \text { TREE } & \square \text { LOCAL TREE } \\ \nabla \text { SHRUB } & \square \text { LOCAL SHRUB } \\ \square \text { GRASS } & \square \text { LOCAL GRASS } \\ \square \text { FORBS } & \square \text { LOCAL FORBS } \\ \square \text { WETLAND } & \square \text { LOCAL WETLAND }\end{array}$

URL: www.georgejohnsonnursery.com 
Harold Poole Nursery

P.O. Box 153

Forest Hill, LA

71430

PHONE: (318) 748-6959

URL: www.haroldpoolesnursery.com

Holloways Nursery, Inc.

Box 339

Forest Hill, LA

71430

PHONE: (318) 748-6803

URL: www.hollawaysnursery.com

\section{L\&R Nursery}

3386 Highway 112

Forest Hill, LA

71430

PHONE: (318) 748-4345

URL: none currently available

Morris \& Sons Nursery

305 Fish Hatchery Rd

Forest Hill, LA

71430

PHONE: (318) 748-7568

URL: www.morrisandsonsnursery.com

Richards Nursery

4061 Highway 112

Forest Hill, LA

71430

PHONE: (318) 748-6587

URL: www.richardsnursery.com

\section{Robert Bates Nursery, Inc.}

Box 100

Forest Hill, LA

71430

PHONE: (318) 748-6791

URL: www.robertbatesnursery.com

$\begin{array}{ll}\nabla \text { TREE } & \square \text { LOCAL TREE } \\ \nabla \text { SHRUB } & \square \text { LOCAL SHRUB } \\ \square \text { GRASS } & \square \text { LOCAL GRASS } \\ \square \text { FORBS } & \square \text { LOCAL FORBS } \\ \square \text { WETLAND } & \square \text { LOCAL WETLAND }\end{array}$

$\begin{array}{ll}\nabla \text { TREE } & \nabla \text { LOCAL TREE } \\ \nabla \text { SHRUB } & \nabla \text { LOCAL SHRUB } \\ \square \text { GRASS } & \square \text { LOCAL GRASS } \\ \square \text { FORBS } & \square \text { LOCAL FORBS } \\ \square \text { WETLAND } & \square \text { LOCAL WETLAND }\end{array}$

$\checkmark$ TREE

$\checkmark$ SHRUB

$\checkmark$ LOCAL TREE

$\checkmark$ LOCAL SHRUB

$\square$ GRASS

$\square$ FORBS

$\square$ LOCAL GRASS

$\square$ LOCAL FORBS

$\square$ WETLAND $\square$ LOCAL WETLAND

$\begin{array}{ll}\square \text { TREE } & \square \text { LOCAL TREE } \\ \square \text { SHRUB } & \square \text { LOCAL SHRUB } \\ \square \text { GRASS } & \square \text { LOCAL GRASS } \\ \square \text { FORBS } & \square \text { LOCAL FORBS } \\ \square \text { WETLAND } & \square \text { LOCAL WETLAND }\end{array}$

$\nabla$ TREE

$\square$ SHRUB

$\square$ GRASS

$\square$ FORBS

$\square$ WETLAND

$\nabla$ LOCAL TREE

$\checkmark$ LOCAL SHRUB

$\square$ LOCAL GRASS

$\square$ LOCAL FORBS

$\square$ LOCAL WETLAND

$\begin{array}{ll}\square \text { TREE } & \square \text { LOCAL TREE } \\ \nabla \text { SHRUB } & \nabla \text { LOCAL SHRUB } \\ \square \text { GRASS } & \square \text { LOCAL GRASS } \\ \square \text { FORBS } & \square \text { LOCAL FORBS } \\ \square \text { WETLAND } & \square \text { LOCAL WETLAND }\end{array}$

Franklinton

Seal Nursery

42174 Seal Rd.

Franklinton, LA

70438

PHONE: (985) 839-5701

$\begin{array}{ll}\nabla \text { TREE } & \square \text { LOCAL TREE } \\ \square \text { SHRUB } & \square \text { LOCAL SHRUB } \\ \square \text { GRASS } & \square \text { LOCAL GRASS } \\ \square \text { FORBS } & \square \text { LOCAL FORBS } \\ \square \text { WETLAND } & \square \text { LOCAL WETLAND }\end{array}$

URL: www.sealnursery.com 
Galliano

Plant Materials Center

438 Airport Rd

Galliano, LA

70354

PHONE: (985) 475-5280

$\begin{array}{ll}\square \text { TREE } & \square \text { LOCAL TREE } \\ \square \text { SHRUB } & \square \text { LOCAL SHRUB } \\ \square \text { GRASS } & \square \text { LOCAL GRASS } \\ \square \text { FORBS } & \square \text { LOCAL FORBS } \\ \square \text { WETLAND } & \square \text { LOCAL WETLAND }\end{array}$

URL: http://plant-materials.nrcs.usda.gov/lapmc/

\section{Grand Coteau}

Barrys Nursery

Box 390

$\nabla$ TREE $\quad \nabla$ LOCAL TREE

Grand Coteau, LA

70541

$\checkmark$ SHRUB

$\checkmark$ LOCAL SHRUB

PHONE: (337) 662-5318

$\square$ GRASS $\square$ LOCAL GRASS

$\square$ FORBS $\square$ LOCAL FORBS

$\square$ WETLAND $\square$ LOCAL WETLAND

URL: none currently available

\section{Jonesboro}

The Bosch Nursery, Inc.

Rt.2, Box 142A"

Jonesboro, LA

71251

PHONE: (318) 259-9484

$\begin{array}{ll}\nabla \text { TREE } & \square \text { LOCAL TREE } \\ \nabla \text { SHRUB } & \square \text { LOCAL SHRUB } \\ \square \text { GRASS } & \square \text { LOCAL GRASS } \\ \square \text { FORBS } & \square \text { LOCAL FORBS } \\ \square \text { WETLAND } & \square \text { LOCAL WETLAND }\end{array}$

URL: none currently available

\section{Lafayette}

Dugals Nursery Wholesale

931 Tolson Rd.

Lafayette, LA

70508

PHONE: (337) 837-1233

URL: none currently available

Teche Nursery \& Landscaping

3326A Verot School Rd

Lafayette, LA

70508

PHONE: (337) 856-5908

URL: none currently available

\section{Lecompte}

Garland Nichols Nursery

3268 Hwy. 112

Lecompte, LA

71346

PHONE: (318) 748-6594

$\begin{array}{ll}\nabla \text { TREE } & \square \text { LOCAL TREE } \\ \square \text { SHRUB } & \square \text { LOCAL SHRUB } \\ \square \text { GRASS } & \square \text { LOCAL GRASS } \\ \square \text { FORBS } & \square \text { LOCAL FORBS } \\ \square \text { WETLAND } & \square \text { LOCAL WETLAND }\end{array}$

$\begin{array}{ll}\nabla \text { TREE } & \square \text { LOCAL TREE } \\ \square \text { SHRUB } & \square \text { LOCAL SHRUB } \\ \square \text { GRASS } & \square \text { LOCAL GRASS } \\ \square \text { FORBS } & \square \text { LOCAL FORBS } \\ \square \text { WETLAND } & \square \text { LOCAL WETLAND }\end{array}$

URL: none currently available

$\begin{array}{ll}\nabla \text { TREE } & \square \text { LOCAL TREE } \\ \square \text { SHRUB } & \square \text { LOCAL SHRUB } \\ \square \text { GRASS } & \square \text { LOCAL GRASS } \\ \square \text { FORBS } & \square \text { LOCAL FORBS } \\ \square \text { WETLAND } & \square \text { LOCAL WETLAND }\end{array}$




$\begin{array}{lll}\text { Louisiana Forest Seed Co. Inc. } & \\ \text { 303 Forestry Rd. } & \square \text { TREE } & \square \text { LOCAL TREE } \\ \text { Lecompte, LA } & \square \text { SHRUB } & \square \text { LOCAL SHRUB } \\ 71346 & \square \text { GRASS } & \square \text { LOCAL GRASS } \\ \text { PHONE: (318) 443-5026 } & \square \text { FORBS } & \square \text { LOCAL FORBS } \\ & \square \text { WETLAND } & \square \text { LOCAL WETLAND }\end{array}$

URL: none currently available

\section{Loranger}

P.J.s Nursery

Star Rt., Box 60"

Loranger, LA

70446

PHONE: (985) 796-9001

$\begin{array}{ll}\square \text { TREE } & \square \text { LOCAL TREE } \\ \square \text { SHRUB } & \square \text { LOCAL SHRUB } \\ \square \text { GRASS } & \square \text { LOCAL GRASS } \\ \square \text { FORBS } & \square \text { LOCAL FORBS } \\ \square \text { WETLAND } & \square \text { LOCAL WETLAND }\end{array}$

URL: none currently available

Straughans Nursery, Inc. 53514 Cook Rd.

Loranger, LA

70446

PHONE: (985) 878-9233

$\begin{array}{ll}\square \text { TREE } & \square \text { LOCAL TREE } \\ \square \text { SHRUB } & \square \text { LOCAL SHRUB } \\ \square \text { GRASS } & \square \text { LOCAL GRASS } \\ \square \text { FORBS } & \square \text { LOCAL FORBS } \\ \square \text { WETLAND } & \square \text { LOCAL WETLAND }\end{array}$

URL: none currently available

$\begin{array}{lll}\text { Marksville } & & \\ \text { Rists Nursery } & & \\ 259 \text { Old Moncla Rd } & \nabla \text { TREE } & \square \text { LOCAL TREE } \\ \text { Marksville, LA } & \square \text { SHRUB } & \square \text { LOCAL SHRUB } \\ 71351 & \square \text { GRASS } & \square \text { LOCAL GRASS } \\ \text { PHONE: (318) 253-7745 } & \square \text { FORBS } & \square \text { LOCAL FORBS } \\ & \square \text { WETLAND } & \square \text { LOCAL WETLAND }\end{array}$

URL: none currently available

Metarie

Don Huemann Greenhouse \& Lab 808 Rue Chartres

$\nabla$ TREE $\quad \nabla$ LOCAL TREE

Metarie, LA

$\nabla$ SHRUB $\quad \nabla$ LOCAL SHRUB

70005

$\checkmark$ GRASS $\nabla$ LOCAL GRASS

PHONE: (504) 833-2473

$\checkmark$ FORBS $\quad \nabla$ LOCAL FORBS

$\nabla$ WETLAND $\nabla$ LOCAL WETLAND

URL: none currently available

Montegut

Theriot \& Brunet Nursery

639 Hwy. 55

Montegut, LA

70377

PHONE: (985) 594-4110

$\begin{array}{ll}\square \text { TREE } & \square \text { LOCAL TREE } \\ \square \text { SHRUB } & \square \text { LOCAL SHRUB } \\ \square \text { GRASS } & \square \text { LOCAL GRASS } \\ \square \text { FORBS } & \square \text { LOCAL FORBS } \\ \square \text { WETLAND } & \square \text { LOCAL WETLAND }\end{array}$

URL: none currently available 
New lberia

Live Oak Gardens, Ltd.

10106 Jefferson Island Rd

New Iberia, LA

70560

PHONE: (800) 725-5625

$\begin{array}{ll}\nabla \text { TREE } & \square \text { LOCAL TREE } \\ \square \text { SHRUB } & \square \text { LOCAL SHRUB } \\ \square \text { GRASS } & \square \text { LOCAL GRASS } \\ \square \text { FORBS } & \square \text { LOCAL FORBS } \\ \square \text { WETLAND } & \square \text { LOCAL WETLAND }\end{array}$

URL: none currently available

\section{New Orleans}

Bahams Nurseries \& Landscape $\mathrm{C}_{0}$

3520 GeneralL De Gaulle Dr

New Orleans, LA

70131

PHONE: (504) 391-0353

$\begin{array}{ll}\nabla \text { TREE } & \nabla \text { LOCAL TREE } \\ \nabla \text { SHRUB } & \nabla \text { LOCAL SHRUB } \\ \nabla \text { GRASS } & \square \text { LOCAL GRASS } \\ \nabla \text { FORBS } & \square \text { LOCAL FORBS } \\ \nabla \text { WETLAND } & \nabla \text { LOCAL WETLAND }\end{array}$

URL: none currently available

\section{Opelousas}

Louisiana Nursery, Inc.

5853 Highway 182

Opelousas, LA

70570

PHONE: (337) 948-3696

$\begin{array}{ll}\nabla \text { TREE } & \square \text { LOCAL TREE } \\ \nabla \text { SHRUB } & \nabla \text { LOCAL SHRUB } \\ \square \text { GRASS } & \square \text { LOCAL GRASS } \\ \square \text { FORBS } & \square \text { LOCAL FORBS } \\ \square \text { WETLAND } & \square \text { LOCAL WETLAND }\end{array}$

URL: www.durionursery.com

\section{Washington}

Johns Wholesale Nursery

3327 Grand Prarie Hwy

Washington, LA

70589

PHONE: (318) 826-7190

$\begin{array}{ll}\nabla \text { TREE } & \square \text { LOCAL TREE } \\ \nabla \text { SHRUB } & \checkmark \text { LOCAL SHRUB } \\ \square \text { GRASS } & \square \text { LOCAL GRASS } \\ \square \text { FORBS } & \square \text { LOCAL FORBS } \\ \square \text { WETLAND } & \square \text { LOCAL WETLAND }\end{array}$

URL: www. ohnswholesalenursery.com

LaFleurs Nursery, Inc.

Star Rt., Box 222A"

Washington, LA

70589

PHONE: (337) 826-7118

$\begin{array}{ll}\nabla \text { TREE } & \square \text { LOCAL TREE } \\ \square \text { SHRUB } & \square \text { LOCAL SHRUB } \\ \square \text { GRASS } & \square \text { LOCAL GRASS } \\ \square \text { FORBS } & \square \text { LOCAL FORBS } \\ \square \text { WETLAND } & \square \text { LOCAL WETLAND }\end{array}$

URL: none currently available

\section{Westwego}

Banting Family Business Farms

3425 River Rd.

Westwego, LA

70094

PHONE: (504) 436-4343

$\begin{array}{ll}\nabla \text { TREE } & \square \text { LOCAL TREE } \\ \nabla \text { SHRUB } & \square \text { LOCAL SHRUB } \\ \nabla \text { GRASS } & \square \text { LOCAL GRASS } \\ \nabla \text { FORBS } & \square \text { LOCAL FORBS } \\ \nabla \text { WETLAND } & \square \text { LOCAL WETLAND }\end{array}$

URL: www.bantingnursery.com 
Nine Mile Point Nursery

2141 River Rd.

Westwego, LA

70094

PHONE: (504) 436-4915

URL: none currently available

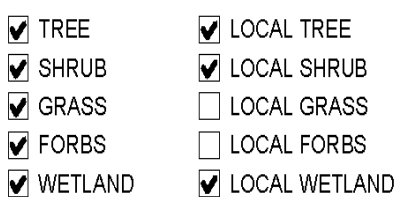

$\begin{array}{ll}\nabla \text { FORBS } & \square \text { LOCAL FORBS } \\ \nabla \text { WETLAND } & \nabla \text { LOCAL WETLAND }\end{array}$

White Castle

Murrell Trading $\mathrm{C}_{0}$, Inc.

30185 Highway 1

White Castle, LA

70788

PHONE: (225) 545-3336

$\begin{array}{ll}\square \text { TREE } & \square \text { LOCAL TREE } \\ \square \text { SHRUB } & \square \text { LOCAL SHRUB } \\ \square \text { GRASS } & \square \text { LOCAL GRASS } \\ \square \text { FORBS } & \square \text { LOCAL FORBS } \\ \square \text { WETLAND } & \square \text { LOCAL WETLAND }\end{array}$

URL: none currently available

\section{Woodworth}

Marshalls Nursery

130 Langston

Woodworth, LA

71485

PHONE: (318) 443-3909

$\begin{array}{ll}\square \text { TREE } & \square \text { LOCAL TREE } \\ \square \text { SHRUB } & \square \text { LOCAL SHRUB } \\ \square \text { GRASS } & \square \text { LOCAL GRASS } \\ \square \text { FORBS } & \square \text { LOCAL FORBS } \\ \square \text { WETLAND } & \square \text { LOCAL WETLAND }\end{array}$

URL: www.marshallsnursery.net

Muse Hill Greenhouse \& Nursery

$\begin{array}{lll}81 \text { Castor Plunge Rd. } & \nabla \text { TREE } & \nabla \text { LOCAL TREE } \\ \text { Woodworth, LA } & \nabla \text { SHRUB } & \nabla \text { LOCAL SHRUB } \\ 71485 & \square \text { GRASS } & \square \text { LOCAL GRASS } \\ \text { PHONE: (800) 473-0666 } & \square \text { FORBS } & \square \text { LOCAL FORBS } \\ & \square \text { WETLAND } & \square \text { LOCAL WETLAND }\end{array}$

URL: none currently available

\section{Massachusetts}

\section{Amherst}

New England Wetland Plants, Inc. 800 Main Street

Amherst, MA

01002

PHONE: (413) 256-1752

$\checkmark$ TREE

$\checkmark$ SHRUB

$\checkmark$ GRASS

$\checkmark$ FORBS

$\checkmark$ WETLAND $\checkmark$ LOCAL TREE $\nabla$ LOCAL SHRUB $\checkmark$ LOCAL GRASS

$\checkmark$ LOCAL FORBS

$\checkmark$ LOCAL WETLAND

URL: www.newp.com

Carlisle

Blanchette Gardens

223 Rutland St

Carlisle, MA

01741

PHONE: (978) 369-2962

URL: none currently available

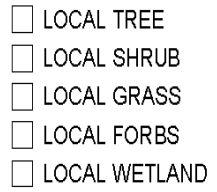

$\square$ WETLAND $\square$ LOCAL WETLAND 
East Falmouth

Mahoneys Too

958 E. Falmouth Hwy

East Falmouth, MA

02536

PHONE: (508) 548-4842

$\begin{array}{ll}\nabla \text { TREE } & \square \text { LOCAL TREE } \\ \nabla \text { SHRUB } & \square \text { LOCAL SHRUB } \\ \nabla \text { GRASS } & \square \text { LOCAL GRASS } \\ \nabla \text { FORBS } & \square \text { LOCAL FORBS } \\ \square \text { WETLAND } & \square \text { LOCAL WETLAND }\end{array}$

URL: none currently available

\section{East Freetown}

\section{Biomass Farms}

15 Mohawk Ave.

East Freetown, MA

02717

PHONE: (508) 763-5253

$$
\begin{array}{ll}
\nabla \text { TREE } & \nabla \text { LOCAL TREE } \\
\nabla \text { SHRUB } & \nabla \text { LOCAL SHRUB } \\
\nabla \text { GRASS } & \square \text { LOCAL GRASS } \\
\nabla \text { FORBS } & \square \text { LOCAL FORBS } \\
\nabla \text { WETLAND } & \nabla \text { LOCAL WETLAND }
\end{array}
$$

URL: www.biofence.com

\section{Edgartown}

Donaromas Nursery

$\begin{array}{lll}271 \text { Upper Main Street } & \nabla \text { TREE } & \square \text { LOCAL TREE } \\ \text { Edgartown, MA } & \nabla \text { SHRUB } & \square \text { LOCAL SHRUB } \\ \text { 02539 } & \square \text { GRASS } & \square \text { LOCAL GRASS } \\ \text { PHONE: (508) 627-8366 } & \square \text { FORBS } & \square \text { LOCAL FORBS } \\ & \nabla \text { WETLAND } & \square \text { LOCAL WETLAND }\end{array}$

URL: www.donaromas.com

\section{Framingham}

New England Wildflower Society

180 Hemmenway Rd.

Framingham, MA

01701-269

PHONE: (508) 877-7630

$\begin{array}{ll}\nabla \text { TREE } & \square \text { LOCAL TREE } \\ \nabla \text { SHRUB } & \checkmark \text { LOCAL SHRUB } \\ \square \text { GRASS } & \checkmark \text { LOCAL GRASS } \\ \nabla \text { FORBS } & \square \text { LOCAL FORBS } \\ \square \text { WETLAND } & \checkmark \text { LOCAL WETLAND }\end{array}$

URL: www.newfs.org

Hadley

Wanczyk Evergreen Nursery, Inc.

166 Russell St.

Hadley, MA

01035

PHONE: (413) 584-3709

$\begin{array}{ll}\nabla \text { TREE } & \square \text { LOCAL TREE } \\ \square \text { SHRUB } & \square \text { LOCAL SHRUB } \\ \square \text { GRASS } & \square \text { LOCAL GRASS } \\ \square \text { FORBS } & \square \text { LOCAL FORBS } \\ \square \text { WETLAND } & \square \text { LOCAL WETLAND }\end{array}$

URL: none currently available

\section{Hopkinton}

Weston Nurseries, Inc.

P.O. Box 186

Hopkinton, MA

01748

PHONE: (508) 435-3414

URL: www.westonnurseries.com

$\begin{array}{ll}\nabla \text { TREE } & \nabla \text { LOCAL TREE } \\ \nabla \text { SHRUB } & \nabla \text { LOCAL SHRUB } \\ \nabla \text { GRASS } & \checkmark \text { LOCAL GRASS } \\ \square \text { FORBS } & \square \text { LOCAL FORBS } \\ \square \text { WETLAND } & \checkmark \text { LOCAL WETLAND }\end{array}$

$\checkmark$ WETLAND $\checkmark$ LOCAL WETLAND 
North Attleboro

Underground Shade Nursery

P.O. Box 1386

North Attleboro, MA

02763

PHONE: (508) 222-2164

$\begin{array}{ll}\square \text { TREE } & \square \text { LOCAL TREE } \\ \square \text { SHRUB } & \square \text { LOCAL SHRUB } \\ \square \text { GRASS } & \square \text { LOCAL GRASS } \\ \nabla \text { FORBS } & \square \text { LOCAL FORBS } \\ \square \text { WETLAND } & \square \text { LOCAL WETLAND }\end{array}$

URL: none currently available

Northboro

Bigelow Nurseries

455 W. Main St.

Northboro, MA

01532

PHONE: (508) 845-2143

$\begin{array}{ll}\nabla \text { TREE } & \nabla \text { LOCAL TREE } \\ \nabla \text { SHRUB } & \checkmark \text { LOCAL SHRUB } \\ \square \text { GRASS } & \square \text { LOCAL GRASS } \\ \nabla \text { FORBS } & \square \text { LOCAL FORBS } \\ \square \text { WETLAND } & \square \text { LOCAL WETLAND }\end{array}$

URL: www.bigelownurseries.com

Sandwich

F. W. Schumacher $\mathrm{C}_{0}$., Inc.

$\begin{array}{lll}36 \text { Spring Hill Rd. } & \square \text { TREE } & \square \text { LOCAL TREE } \\ \text { Sandwich, MA } & \square \text { SHRUB } & \square \text { LOCAL SHRUB } \\ 02563 & \square \text { GRASS } & \square \text { LOCAL GRASS } \\ \text { PHONE: (508) 888-0659 } & \square \text { FORBS } & \square \text { LOCAL FORBS } \\ & \square \text { WETLAND } & \square \text { LOCAL WETLAND }\end{array}$

URL: www.treeshrubseeds.com

\section{South Westport}

Sylvan Nursery, Inc.

1028 Horseneck Rd.

$\nabla$ TREE $\quad \square$ LOCAL TREE

South Westport. MA

02790

PHONE: (508) 636-5615

$\nabla$ SHRUB $\quad \nabla$ LOCAL SHRUB

$\square$ GRASS $\square$ LOCAL GRASS

$\checkmark$ FORBS $\square$ LOCAL FORBS

$\checkmark$ WETLAND $\square$ LOCAL WETLAND

URL: www.sylvannursery.com

\section{Southhampton}

Tripple Brook Farm

37 Middle Rd.

Southhampton, MA

01073

PHONE: (413) 527-4626

$\begin{array}{ll}\nabla \text { TREE } & \square \text { LOCAL TREE } \\ \nabla \text { SHRUB } & \square \text { LOCAL SHRUB } \\ \nabla \text { GRASS } & \square \text { LOCAL GRASS } \\ \nabla \text { FORBS } & \square \text { LOCAL FORBS } \\ \nabla \text { WETLAND } & \nabla \text { LOCAL WETLAND }\end{array}$

URL: www.tripplebrookfarm.com

\section{Westford}

Laughton Nursery

31 Lowell Rd

$\nabla$ TREE

Westford, MA

01886

PHONE: (978) 692-7752

$\checkmark$ SHRUB

$\checkmark$ LOCAL TREE

$\square$ GRASS

$\checkmark$ LOCAL SHRUB

$\square$ FORBS $\square$ LOCAL FORBS

$\checkmark$ WETLAND $\square$ LOCAL WETLAND

URL: www.laughtonhort.com 
Williamstown

Gardeners Marketplace

208 Water Street

Williamstown, MA

01267

PHONE: (800) 359-7436

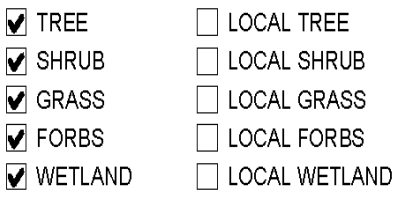

URL: http:/Wwww.gardenersmarketplace.com/

\section{Maryland}

Baldwin

Kurt Bluemel, Inc.

2740 Greene Lane

Baldwin, MD

21013

PHONE: (410) 557-7229

$\begin{array}{ll}\square \text { TREE } & \square \text { LOCAL TREE } \\ \square \text { SHRUB } & \square \text { LOCAL SHRUB } \\ \square \text { GRASS } & \square \text { LOCAL GRASS } \\ \square \text { FORBS } & \square \text { LOCAL FORBS } \\ \square \text { WETLAND } & \square \text { LOCAL WETLAND }\end{array}$

URL: www.kurtbluemel.com

\section{Baltimore}

Landreth Seed $\mathrm{C}_{0}$.

180 W. Ostend St.

Baltimore, MD

21230

PHONE: (410) 727-3922

$\begin{array}{ll}\square \text { TREE } & \square \text { LOCAL TREE } \\ \square \text { SHRUB } & \square \text { LOCAL SHRUB } \\ \square \text { GRASS } & \square \text { LOCAL GRASS } \\ \square \text { FORBS } & \square \text { LOCAL FORBS } \\ \square \text { WETLAND } & \square \text { LOCAL WETLAND }\end{array}$

URL: www.landrethseeds.com

\section{Maryland Natives Nursery}

9120 Hines Rd

Baltimore, MD

21234

PHONE: (410) 529-0552

$\begin{array}{ll}\nabla \text { TREE } & \nabla \text { LOCAL TREE } \\ \nabla \text { SHRUB } & \nabla \text { LOCAL SHRUB } \\ \nabla \text { GRASS } & \nabla \text { LOCAL GRASS } \\ \nabla \text { FORBS } & \square \text { LOCAL FORBS } \\ \nabla \text { WETLAND } & \square \text { LOCAL WETLAND }\end{array}$

URL: www.marylandnativesnursery.com

Wickleins Water Gardens

1820 Cromwell Bridge Rd.

Baltimore, MD

21234

PHONE: (410) 823-1335

$\begin{array}{ll}\square \text { TREE } & \square \text { LOCAL TREE } \\ \square \text { SHRUB } & \square \text { LOCAL SHRUB } \\ \square \text { GRASS } & \square \text { LOCAL GRASS } \\ \square \text { FORBS } & \square \text { LOCAL FORBS } \\ \square \text { WETLAND } & \square \text { LOCAL WETLAND }\end{array}$

URL: www.wickleinaquatics.com

\section{Beltsville}

Plant Materials Center

Bldg. 509, BARC-East, E. Beaver Dam Rd.

Beltsville, MD

20705

PHONE: (301) 504-8175

$\begin{array}{ll}\square \text { TREE } & \square \text { LOCAL TREE } \\ \square \text { SHRUB } & \square \text { LOCAL SHRUB } \\ \square \text { GRASS } & \square \text { LOCAL GRASS } \\ \square \text { FORBS } & \square \text { LOCAL FORBS } \\ \square \text { WETLAND } & \square \text { LOCAL WETLAND }\end{array}$

URL: http://plant-materials.nrcs.usda.gov/mdpmc/ 
Buckeystown

Lilypons Water Gardens

6800 Lilypons Rd.

Buckeystown, MD

21717

PHONE: (800) 999-5499

$\begin{array}{ll}\square \text { TREE } & \square \text { LOCAL TREE } \\ \square \text { SHRUB } & \square \text { LOCAL SHRUB } \\ \square \text { GRASS } & \square \text { LOCAL GRASS } \\ \square \text { FORBS } & \square \text { LOCAL FORBS } \\ \square \text { WETLAND } & \square \text { LOCAL WETLAND }\end{array}$

URL: www.lilypons.com

\section{Chesapeake City}

Mloon Nursery

P.O. Box 672

Chesapeake City, MD

21915

PHONE: (410) 755-6600

$\begin{array}{ll}\nabla \text { TREE } & \square \text { LOCAL TREE } \\ \square \text { SHRUB } & \square \text { LOCAL SHRUB } \\ \square \text { GRASS } & \square \text { LOCAL GRASS } \\ \square \text { FORBS } & \square \text { LOCAL FORBS } \\ \square \text { WETLAND } & \square \text { LOCAL WETLAND }\end{array}$

URL: www.moonnurseries.com

Dayton

Native Seeds, Inc.

14590 Triadelphia Mill Rd.

Dayton, MD

21036

PHONE: (301) 596-9818

$\begin{array}{ll}\square \text { TREE } & \square \text { LOCAL TREE } \\ \square \text { SHRUB } & \square \text { LOCAL SHRUB } \\ \square \text { GRASS } & \square \text { LOCAL GRASS } \\ \square \text { FORBS } & \square \text { LOCAL FORBS } \\ \square \text { WETLAND } & \square \text { LOCAL WETLAND }\end{array}$

URL: none currently available

\section{Forest Hill}

Atlantic Star Nursery

620 Pyle Rd.

Forest Hill, MD

21050

PHONE: (410) 838-7950

URL: none currently available

Conebrook Nursery

P.O. Box 177

Forest Hill, MD

21050

PHONE: (410) 838-4747

URL: none currently available

\section{Jarrettsville}

Maryland Aquatic Nurseries

3427 N. Furnace Rd.

Jarrettsville, MD

21084

PHONE: (410) 557-7615

$\begin{array}{ll}\square \text { TREE } & \square \text { LOCAL TREE } \\ \square \text { SHRUB } & \square \text { LOCAL SHRUB } \\ \square \text { GRASS } & \square \text { LOCAL GRASS } \\ \square \text { FORBS } & \square \text { LOCAL FORBS } \\ \square \text { WETLAND } & \square \text { LOCAL WETLAND }\end{array}$

$\nabla$ TREE $\quad \nabla$ LOCAL TREE

$\nabla$ SHRUB $\nabla$ LOCAL SHRUB

$\square$ GRASS $\square$ LOCAL GRASS

$\square$ FORBS $\square$ LOCAL FORBS

$\square$ WETLAND $\square$ LOCAL WETLAND

URL: none currently available 


\section{Kennedyville}

Angelica Nurseries, Inc.

11129 Locust Grove Rd.

Kennedyille, MD

21645

PHONE: (410) 928-3111

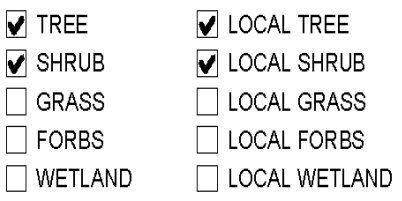

URL: www.angelicanurseries.com

\section{Monkton}

Bluemount Nurseries

2103 Blue Mount Rd.

Monkton, MD

21111

PHONE: (410) 329-6226

$\begin{array}{ll}\square \text { TREE } & \square \text { LOCAL TREE } \\ \square \text { SHRUB } & \square \text { LOCAL SHRUB } \\ \square \text { GRASS } & \square \text { LOCAL GRASS } \\ \square \text { FORBS } & \square \text { LOCAL FORBS } \\ \square \text { WETLAND } & \square \text { LOCAL WETLAND }\end{array}$

URL: www.bluemount.com

Heartwood Nursery, Inc.

2121 Bluemount Rd

Monkton, MD

21111

PHONE: (410) 343-0390

$\begin{array}{ll}\nabla \text { TREE } & \square \text { LOCAL TREE } \\ \nabla \text { SHRUB } & \square \text { LOCAL SHRUB } \\ \square \text { GRASS } & \square \text { LOCAL GRASS } \\ \square \text { FORBS } & \square \text { LOCAL FORBS } \\ \nabla \text { WETLAND } & \nabla \text { LOCAL WETLAND }\end{array}$

URL: none currently available

Preston

Maryland State Forest Tree Nursery

3424 Gallagher Road

Preston, MD

21655

PHONE: (800) 873-3763

$\begin{array}{ll}\nabla \text { TREE } & \square \text { LOCAL TREE } \\ \nabla \text { SHRUB } & \nabla \text { LOCAL SHRUB } \\ \square \text { GRASS } & \square \text { LOCAL GRASS } \\ \square \text { FORBS } & \square \text { LOCAL FORBS } \\ \square \text { WETLAND } & \square \text { LOCAL WETLAND }\end{array}$

URL: http://Www.dnr.state.md.us/forests/nursery/

\section{Pylesville}

Kollar Nursery

5200 West Heaps Rd.

Pylesville, MD

21132

PHONE: (410) 836-0500

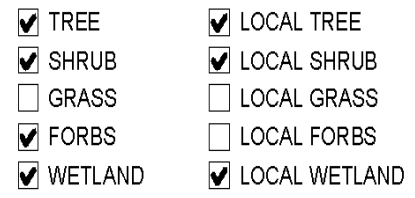

URL: none currently available

\section{Rhodesdale}

William Harper

4645 ENM-Rhodesdale Rd.

Rhodesdale, MD

21659

PHONE: (410) 943-4173

$\begin{array}{ll}\square \text { TREE } & \square \text { LOCAL TREE } \\ \square \text { SHRUB } & \square \text { LOCAL SHRUB } \\ \square \text { GRASS } & \square \text { LOCAL GRASS } \\ \square \text { FORBS } & \square \text { LOCAL FORBS } \\ \square \text { WETLAND } & \square \text { LOCAL WETLAND }\end{array}$

URL: none currently available 
Ridgely

Adkins Arboretum Ltd.

$\begin{array}{lll}\text { PO Box } 100 & \nabla \text { TREE } & \nabla \text { LOCAL TREE } \\ \text { Ridgely, MD } & \nabla \text { SHRUB } & \nabla \text { LOCAL SHRUB } \\ 21660 & \nabla \text { GRASS } & \boldsymbol{\nabla} \text { LOCAL GRASS } \\ \text { PHONE: (410) 634-2847 } & \nabla \text { FORBS } & \mathbf{v} \text { LOCAL FORBS } \\ & \nabla \text { WETLAND } & \nabla \text { LOCAL WETLAND }\end{array}$

URL: www.adkinsarboretum.org

Rockville

Newsom Seed, Inc.

14 Derwood Circle

Rockville, MD

20850

PHONE: (301) 762-2096

$\begin{array}{ll}\square \text { TREE } & \square \text { LOCAL TREE } \\ \square \text { SHRUB } & \square \text { LOCAL SHRUB } \\ \square \text { GRASS } & \square \text { LOCAL GRASS } \\ \square \text { FORBS } & \square \text { LOCAL FORBS } \\ \square \text { WETLAND } & \square \text { LOCAL WETLAND }\end{array}$

URL: www.newsomseed.com

\section{Salisbury}

Chesapeake Nurseries, Inc.

Pemberton Dr.

Salisbury, MD

27571

PHONE: (410) 742-5622

$\begin{array}{ll}\nabla \text { TREE } & \square \text { LOCAL TREE } \\ \nabla \text { SHRUB } & \square \text { LOCAL SHRUB } \\ \square \text { GRASS } & \square \text { LOCAL GRASS } \\ \square \text { FORBS } & \square \text { LOCAL FORBS } \\ \square \text { WETLAND } & \square \text { LOCAL WETLAND }\end{array}$

URL: www.chesapeakenurseries.com

St. Michaels

Environmental Concern, Inc.

POBox P

$\boldsymbol{\nabla}$ TREE $\quad \boldsymbol{\nabla}$ LOCAL TREE

St. Michaels, MD

21663

PHONE: (410) 745-9620

$\nabla$ SHRUB $\quad \nabla$ LOCAL SHRUB

$\square$ GRASS $\square$ LOCAL GRASS

$\checkmark$ FORBS $\quad \square$ LOCAL FORBS

$\checkmark$ WETLAND $\square$ LOCAL WETLAND

URL: www.wetland.org

Street

Foxborough Nursery, Inc.

3611 Miller Rd.

Street, MD

21154

PHONE: (410) 836-7023

$\begin{array}{ll}\nabla \text { TREE } & \nabla \text { LOCAL TREE } \\ \square \text { SHRUB } & \square \text { LOCAL SHRUB } \\ \square \text { GRASS } & \square \text { LOCAL GRASS } \\ \square \text { FORBS } & \square \text { LOCAL FORBS } \\ \square \text { WETLAND } & \square \text { LOCAL WETLAND }\end{array}$

URL: www.foxboroughnursery.com

\section{Taneytown}

J. Christopher Batten, Inc./ Fiddlers Green Nursery 3907 Old Taneytown Rd

Taneytown, MD

21787

PHONE: (410) 751-0424

$\begin{array}{ll}\nabla \text { TREE } & \square \text { LOCAL TREE } \\ \nabla \text { SHRUB } & \square \text { LOCAL SHRUB } \\ \square \text { GRASS } & \square \text { LOCAL GRASS } \\ \square \text { FORBS } & \square \text { LOCAL FORBS } \\ \square \text { WETLAND } & \square \text { LOCAL WETLAND }\end{array}$

URL: none currently available 


\section{Union Bridge}

Clear Ridge Nursery

217 Clear Ridge Rd.

Union Bridge, MD

21791

PHONE: (410) 775-7700

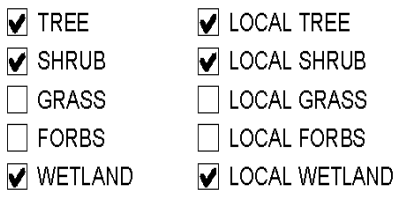

URL: none currently available

\section{Westminister}

\section{Carroll Gardens}

444 E. Main St.

Westminister, MD

21157

PHONE: (800) 638-6334

$\begin{array}{ll}\nabla \text { TREE } & \nabla \text { LOCAL TREE } \\ \nabla \text { SHRUB } & \square \text { LOCAL SHRUB } \\ \nabla \text { GRASS } & \square \text { LOCAL GRASS } \\ \nabla \text { FORBS } & \square \text { LOCAL FORBS } \\ \square \text { WETLAND } & \square \text { LOCAL WETLAND }\end{array}$

URL: www.carrollgardens.com

\section{Maine}

Biddeford

Pierson Nurseries

$\begin{array}{lll}\text { 24 Buzzell Rd } & \nabla \text { TREE } & \square \text { LOCAL TREE } \\ \text { Biddeford, ME } & \square \text { SHRUB } & \square \text { LOCAL SHRUB } \\ \text { 04005 } & \square \text { GRASS } & \square \text { LOCAL GRASS } \\ \text { PHONE: (207) 499-2994 } & \square \text { FORBS } & \square \text { LOCAL FORBS } \\ & \square \text { WETLAND } & \square \text { LOCAL WETLAND }\end{array}$

URL: www.piersonnurseries.com

\section{Fryeburg}

Western Mlaine Nurseries

P.O. Box 250

$\checkmark$ TREE $\quad \nabla$ LOCAL TREE

Fryeburg, ME

04037

PHONE: (800) 447-4745

$\nabla$ SHRUB $\quad \nabla$ LOCAL SHRUB

$\square$ GRASS $\square$ LOCAL GRASS

$\square$ FORBS $\square$ LOCAL FORBS

$\square$ WETLAND $\square$ LOCAL WETLAND

URL: www.westernmainenurseries.com

\section{Vassalboro}

Fieldstone Gardens, Inc.

620 Quaker Lane

Vassalboro, ME

04989-971

PHONE: (207) 923-3836

$\begin{array}{ll}\square \text { TREE } & \square \text { LOCAL TREE } \\ \square \text { SHRUB } & \square \text { LOCAL SHRUB } \\ \square \text { GRASS } & \square \text { LOCAL GRASS } \\ \square \text { FORBS } & \square \text { LOCAL FORBS } \\ \square \text { WETLAND } & \square \text { LOCAL WETLAND }\end{array}$

URL: www.fieldstonegardens.com

\section{Winslow}

Johnnys Selected Seed

955 Benton Ave

Winslow, ME

04901

PHONE: (207) 861-3900

$\begin{array}{ll}\square \text { TREE } & \square \text { LOCAL TREE } \\ \square \text { SHRUB } & \square \text { LOCAL SHRUB } \\ \square \text { GRASS } & \square \text { LOCAL GRASS } \\ \square \text { FORBS } & \square \text { LOCAL FORBS } \\ \square \text { WETLAND } & \square \text { LOCAL WETLAND }\end{array}$

URL: www.johnnyseeds.com 


\section{Michigan}

Allendale

Boschs Country View Nursery, Inc.

$\begin{array}{lll}\text { 10785-84th Ave. } & \nabla \text { TREE } & \square \text { LOCAL TREE } \\ \text { Allendale, MI } & \nabla \text { SHRUB } & \square \text { LOCAL SHRUB } \\ 49401 & \square \text { GRASS } & \square \text { LOCAL GRASS } \\ \text { PHONE: (616) 892-4090 } & \square \text { FORBS } & \square \text { LOCAL FORBS } \\ & \square \text { WETLAND } & \square \text { LOCAL WETLAND }\end{array}$

URL: none currently available

\section{Ann Arbor}

Abbotts Nursery

2781 Scio Church Ro

Ann Arbor, MI

48103

PHONE: (734) 665-8733

$\begin{array}{ll}\nabla \text { TREE } & \square \text { LOCAL TREE } \\ \nabla \text { SHRUB } & \nabla \text { LOCAL SHRUB } \\ \nabla \text { GRASS } & \square \text { LOCAL GRASS } \\ \nabla \text { FORBS } & \square \text { LOCAL FORBS } \\ \square \text { WETLAND } & \square \text { LOCAL WETLAND }\end{array}$

URL: none currently available

Ladys Mantle Nursery

1235 Morningside

Ann Arbor, Ml

48103

PHONE: (734) 769-7304

$\begin{array}{ll}\square \text { TREE } & \square \text { LOCAL TREE } \\ \square \text { SHRUB } & \square \text { LOCAL SHRUB } \\ \square \text { GRASS } & \square \text { LOCAL GRASS } \\ \square \text { FORBS } & \square \text { LOCAL FORBS } \\ \square \text { WETLAND } & \square \text { LOCAL WETLAND }\end{array}$

URL: none currently available

The Native Plant Nursery

P.O. Box 7841

Ann Arbor, Ml

48107

$\checkmark$ TREE

$\checkmark$ SHRUB

$\nabla$ GRASS

$\checkmark$ FORBS

$\nabla$ WETLAND

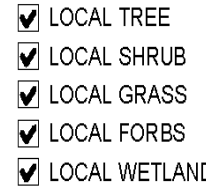

URL: www.nativeplant.com

\section{Berrien Springs}

Twixwood Nursery

4669 E. Hillcrest Dr.

Berrien Springs, MI

49103

PHONE: (269) 471-7408

$\begin{array}{ll}\square \text { TREE } & \square \text { LOCAL TREE } \\ \nabla \text { SHRUB } & \square \text { LOCAL SHRUB } \\ \square \text { GRASS } & \square \text { LOCAL GRASS } \\ \square \text { FORBS } & \square \text { LOCAL FORBS } \\ \square \text { WETLAND } & \square \text { LOCAL WETLAND }\end{array}$

URL: wnw.twixwood.com

China

Marine City Nursery $\mathrm{C}_{0}$.

5304 Marine City Hwy.

China, MI

48054

PHONE: (810) 765-5533

$\begin{array}{ll}\nabla \text { TREE } & \square \text { LOCAL TREE } \\ \nabla \text { SHRUB } & \square \text { LOCAL SHRUB } \\ \square \text { GRASS } & \square \text { LOCAL GRASS } \\ \square \text { FORBS } & \square \text { LOCAL FORBS } \\ \square \text { WETLAND } & \square \text { LOCAL WETLAND }\end{array}$

URL: www.marinecitynursery.com 
Cooks

Northwoods Greenhouse

$\begin{array}{lll}\text { Rt } 1, \text { Box } 97 & \square \text { TREE } & \square \text { LOCAL TREE } \\ \text { Cooks, Ml } & \square \text { SHRUB } & \square \text { LOCAL SHRUB } \\ 49817 & \square \text { GRASS } & \square \text { LOCAL GRASS } \\ \text { PHONE: }(800) \text { 730-4769 } & \square \text { FORBS } & \square \text { LOCAL FORBS } \\ & \square \text { WETLAND } & \square \text { LOCAL WETLAND }\end{array}$

URL: none currently available

Fennville

Wavecrest Nursery \& Landscaping $\mathrm{Co}_{0}$

2509 Lakeshore Dr

$\nabla$ TREE $\quad \nabla$ LOCAL TREE

Fennville, Ml

$\checkmark$ SHRUB $\quad \nabla$ LOCAL SHRUB

49408

$\square$ GRASS $\square$ LOCAL GRASS

PHONE: (269) 543-4175

$\square$ FORBS $\square$ LOCAL FORBS

$\square$ WETLAND $\square$ LOCAL WETLAND

URL: www.wavecrestnursery.com

Free Soil

Cold Stream Farm

2030 Free Soil Rd

Free Soil, MI

49411

PHONE: (616) 464-5809

$\begin{array}{ll}\nabla \text { TREE } & \square \text { LOCAL TREE } \\ \nabla \text { SHRUB } & \square \text { LOCAL SHRUB } \\ \square \text { GRASS } & \square \text { LOCAL GRASS } \\ \square \text { FORBS } & \square \text { LOCAL FORBS } \\ \square \text { WETLAND } & \square \text { LOCAL WETLAND }\end{array}$

URL: none currently available

\section{Grand Haven}

John Zelenka Evergreen Nursery

16127 Winans

Grand Haven, MI

49417

PHONE: (616) 842-1367

$\begin{array}{ll}\square \text { TREE } & \square \text { LOCAL TREE } \\ \nabla \text { SHRUB } & \square \text { LOCAL SHRUB } \\ \square \text { GRASS } & \square \text { LOCAL GRASS } \\ \square \text { FORBS } & \square \text { LOCAL FORBS } \\ \square \text { WETLAND } & \square \text { LOCAL WETLAND }\end{array}$

URL: wWw.zelenkanursery.com

\section{Grand Ledge}

Michigan State Seed Solutions

717 N. Clinton St.

Grand Ledge, MI

48837

PHONE: (517) 627-2164

$\begin{array}{ll}\square \text { TREE } & \square \text { LOCAL TREE } \\ \square \text { SHRUB } & \square \text { LOCAL SHRUB } \\ \square \text { GRASS } & \square \text { LOCAL GRASS } \\ \square \text { FORBS } & \square \text { LOCAL FORBS } \\ \square \text { WETLAND } & \square \text { LOCAL WETLAND }\end{array}$

URL: wWw.seedsolutions.com

\section{Holland}

New Life Nursery, Inc.

3720 - 64th St

$\nabla$ TREE $\quad \square$ LOCAL TREE

Holland, MI

49423

$\checkmark$ SHRUB

$\square$ LOCAL SHRUB

PHONE: (269) 857-1209

$\square$ GRASS

$\square$ LOCAL GRASS

$\square$ FORBS

$\square$ LOCAL FORBS

$\square$ WETLAND $\square$ LOCAL WETLAND

URL: www.newlifenursery.com 
Walters Seed $\mathrm{Co}$.

4645 62nd St.

Holland, MI

49423

PHONE: (616) 335-3282

$\begin{array}{ll}\square \text { TREE } & \square \text { LOCAL TREE } \\ \square \text { SHRUB } & \square \text { LOCAL SHRUB } \\ \square \text { GRASS } & \square \text { LOCAL GRASS } \\ \square \text { FORBS } & \square \text { LOCAL FORBS } \\ \square \text { WETLAND } & \square \text { LOCAL WETLAND }\end{array}$

URL: www.walters-seed.com

\section{Kalamazoo}

Nesta Prairie Perennials

$\begin{array}{lll}1019 \text { Miller Rd. } & \nabla \text { TREE } & \nabla \text { LOCAL TREE } \\ \text { Kalamazoo, MI } & \nabla \text { SHRUB } & \nabla \text { LOCAL SHRUB } \\ \text { 49001 } & \nabla \text { GRASS } & \nabla \text { LOCAL GRASS } \\ \text { PHONE: (800) 233-5025 } & \nabla \text { FORBS } & \nabla \text { LOCAL FORBS } \\ & \nabla \text { WETLAND } & \mathbf{v} \text { LOCAL WETLAND }\end{array}$

URL: none currently available

Oikos Tree Crops

PO Box 19425

Kalamazoo, MI

49019

PHONE: (269) 624-6233

$\begin{array}{ll}\square \text { TREE } & \square \text { LOCAL TREE } \\ \square \text { SHRUB } & \square \text { LOCAL SHRUB } \\ \square \text { GRASS } & \square \text { LOCAL GRASS } \\ \square \text { FORBS } & \square \text { LOCAL FORBS } \\ \square \text { WETLAND } & \square \text { LOCAL WETLAND }\end{array}$

URL: www.oikostreecrops.com

\section{Livington}

Needlefast Evergreen, Inc.

4075 W Hansen Rd

Livington, Ml

49431

$\boldsymbol{v}$ TREE

$\checkmark$ SHRUB

$\checkmark$ LOCAL TREE

PHONE: (231) 843-8524

$\square$ GRASS

LOCAL SHRUB

$\square$ FORBS

$\square$ LOCAL GRASS

$\checkmark$ WETLAND

$\square$ LOCAL FORBS

URL: www.needlefastevergreen.com

Locota

Hartmann Plant Company

PO Box 100

Locota, MI

49063

PHONE: (616) 253-4281

$\begin{array}{ll}\square \text { TREE } & \square \text { LOCAL TREE } \\ \square \text { SHRUB } & \square \text { LOCAL SHRUB } \\ \square \text { GRASS } & \square \text { LOCAL GRASS } \\ \square \text { FORBS } & \square \text { LOCAL FORBS } \\ \square \text { WETLAND } & \square \text { LOCAL WETLAND }\end{array}$

URL: www.hartmannsplantcompany.com

$\begin{array}{lll}\text { Manistee } & & \\ \text { Hramor Nursery } & & \\ 515 \text { 9th St. } & \nabla \text { TREE } & \square \text { LOCAL TREE } \\ \text { Manistee, Ml } & \nabla \text { SHRUB } & \square \text { LOCAL SHRUB } \\ \text { 49660 } & \square \text { GRASS } & \square \text { LOCAL GRASS } \\ \text { PHONE: (231) 723-4846 } & \square \text { FORBS } & \square \text { LOCAL FORBS } \\ & \square \text { WETLAND } & \square \text { LOCAL WETLAND }\end{array}$

URL: none currently available 
Mason

WILDTYPE Native Plants

900 North Every Rd.

Mason, Ml

48854

$\checkmark$ LOCAL TREE

PHONE: (517) 244-1140

$\checkmark$ SHRUB $\quad \nabla$ LOCAL SHRUB

$\checkmark$ GRASS $\square$ LOCAL GRASS

$\nabla$ FORBS $\quad \nabla$ LOCAL FORBS

$\nabla$ WETLAND $\square$ LOCAL WETLAND

URL: www.wildtypeplants.com

\section{Mount Pleasant}

Sweeney Seed Co.

$110 \mathrm{~S}$. Washington St

$\begin{array}{ll}\nabla \text { TREE } & \square \text { LOCAL TREE } \\ \nabla \text { SHRUB } & \square \text { LOCAL SHRUB } \\ \square \text { GRASS } & \square \text { LOCAL GRASS } \\ \nabla \text { FORBS } & \square \text { LOCAL FORBS } \\ \square \text { WETLAND } & \square \text { LOCAL WETLAND }\end{array}$

Mount Pleasant, M

48858

PHONE: (989) 773-5391

$\square$ WETLAND $\square$ LOCAL WETLAND

URL: none currently available

Munising

Coryell Nurseries, Inc.

$\begin{array}{lll}\text { Rt.1, Box 810" } & \nabla \text { TREE } & \square \text { LOCAL TREE } \\ \text { Munising, Ml } & \square \text { SHRUB } & \square \text { LOCAL SHRUB } \\ 49862 & \square \text { GRASS } & \square \text { LOCAL GRASS } \\ \text { PHONE: (906) 387-4356 } & \square \text { FORBS } & \square \text { LOCAL FORBS } \\ & \square \text { WETLAND } & \square \text { LOCAL WETLAND }\end{array}$

URL: none currently available

Muskegon

Ole Henry Gardens

665 W Broadway

Muskegon, MI

49441

$\square$ TREE $\quad \square$ LOCAL TREE

$\checkmark$ SHRUB

$\square$ LOCAL SHRUB

PHONE: (231) 733-1876

$\checkmark$ GRASS

$\square$ LOCAL GRASS

$\checkmark$ FORBS $\square$ LOCAL FORBS

$\square$ WETLAND $\square$ LOCAL WETLAND

URL: none currently available

Portland

The Michigan Wildflower Farm

11770 Cutler Rd.

Portland, MI

48875

PHONE: (517) 647-6010

$\begin{array}{ll}\square \text { TREE } & \square \text { LOCAL TREE } \\ \square \text { SHRUB } & \square \text { LOCAL SHRUB } \\ \square \text { GRASS } & \square \text { LOCAL GRASS } \\ \nabla \text { FORBS } & \square \text { LOCAL FORBS } \\ \square \text { WETLAND } & \square \text { LOCAL WETLAND }\end{array}$

URL: wWw.michiganwildflowerfarm.com

\section{Richmond}

D \& B Plants

27550 School Section Rd

$\square$ TREE

Richmond, M

48062

PHONE: (810) 392-3393

$\checkmark$ SHRUB

$\square$ GRASS

$\square$ LOCAL TREE

$\square$ GRASS

$\square$ LOCAL SHRUB

$\checkmark$ WETLAND $\square$ LOCAL WETLAND

URL: none currently available 


\section{Saginaw}

Wetlands Nursery

P.O. Box 14553

Saginaw, MI

48601

PHONE: (517) 752-3492

$\begin{array}{ll}\square \text { TREE } & \square \text { LOCAL TREE } \\ \square \text { SHRUB } & \square \text { LOCAL SHRUB } \\ \square \text { GRASS } & \square \text { LOCAL GRASS } \\ \square \text { FORBS } & \square \text { LOCAL FORBS } \\ \square \text { WETLAND } & \square \text { LOCAL WETLAND }\end{array}$

URL: www.wetlands-nursery.com

\section{South Lyon}

Red Fox Conservation Plants \& Seeds

PO Box 306

South Lyon, MI

48178

PHONE: (248) 486-1127

$\begin{array}{ll}\nabla \text { TREE } & \square \text { LOCAL TREE } \\ \nabla \text { SHRUB } & \square \text { LOCAL SHRUB } \\ \nabla \text { GRASS } & \square \text { LOCAL GRASS } \\ \nabla \text { FORBS } & \square \text { LOCAL FORBS } \\ \nabla \text { WETLAND } & \square \text { LOCAL WETLAND }\end{array}$

URL: none currently available

\section{West Olive}

Vans Pines, Inc.

7550 144th Ave

West Olive, MI

49460

PHONE: (616) 399-1620

$\begin{array}{ll}\nabla \text { TREE } & \square \text { LOCAL TREE } \\ \nabla \text { SHRUB } & \square \text { LOCAL SHRUB } \\ \square \text { GRASS } & \square \text { LOCAL GRASS } \\ \square \text { FORBS } & \square \text { LOCAL FORBS } \\ \square \text { WETLAND } & \square \text { LOCAL WETLAND }\end{array}$

URL: www.vanspinesnursery.com

Zeeland

Walters Gardens, Inc.

Box 137

$\square$ TREE

Zeeland, M

49464

$\square$ SHRUB

$\square$ GRASS

PHONE: (616) 772-4697

$\checkmark$ FORBS

$\square$ WETLAND

$\square$ LOCAL TREE

$\square$ LOCAL SHRUB

$\square$ LOCAL GRASS

$\square$ LOCAL FORBS

URL: www.waltersgardens.com

\section{Minnesota}

Albert Lea

Albert Lea Seed House

P.O. Box 127

Albert Lea, MN

56007

PHONE: (507) 373-3161

$\begin{array}{ll}\square \text { TREE } & \square \text { LOCAL TREE } \\ \square \text { SHRUB } & \square \text { LOCAL SHRUB } \\ \square \text { GRASS } & \square \text { LOCAL GRASS } \\ \square \text { FORBS } & \square \text { LOCAL FORBS } \\ \square \text { WETLAND } & \square \text { LOCAL WETLAND }\end{array}$

URL: www.alseed.com

\section{Barnum}

Booming Native Plants

$2323 \mathrm{CR} 6$

Barnum, MN

55707

PHONE: (218) 389-3220

$\begin{array}{ll}\square \text { TREE } & \square \text { LOCAL TREE } \\ \square \text { SHRUB } & \square \text { LOCAL SHRUB } \\ \nabla \text { GRASS } & \square \text { LOCAL GRASS } \\ \square \text { FORBS } & \square \text { LOCAL FORBS } \\ \square \text { WETLAND } & \square \text { LOCAL WETLAND }\end{array}$

URL: none currently available 
Battle Lake

Swedberg Nurseries, Inc.

Box 418

Battle Lake, MN

56515

PHONE: (218) 864-5526

$\begin{array}{ll}\nabla \text { TREE } & \square \text { LOCAL TREE } \\ \nabla \text { SHRUB } & \square \text { LOCAL SHRUB } \\ \square \text { GRASS } & \square \text { LOCAL GRASS } \\ \square \text { FORBS } & \square \text { LOCAL FORBS } \\ \square \text { WETLAND } & \square \text { LOCAL WETLAND }\end{array}$

URL: none currently available

Bemidji

Norfarm Seeds, Inc.

Box 725

Bemidji, MN

56619

$\square$ SHRUB

$\square$ LOCAL TREE

$\square$ GRASS

$\square$ LOCAL SHRUB

PHONE: (218) 751-8617

$\square$ FORBS $\square$ LOCAL FORBS

$\square$ WETLAND $\square$ LOCAL WETLAND

URL: none currently available

Blaine

Rice Creek Gardens, Inc.

11506 Highway 65

Blaine, MN

55434

PHONE: (763) 754-8090

$\begin{array}{ll}\nabla \text { TREE } & \square \text { LOCAL TREE } \\ \square \text { SHRUB } & \square \text { LOCAL SHRUB } \\ \square \text { GRASS } & \square \text { LOCAL GRASS } \\ \square \text { FORBS } & \square \text { LOCAL FORBS } \\ \square \text { WETLAND } & \square \text { LOCAL WETLAND }\end{array}$

URL: www.ricecreekgardens.com

\section{Blue Earth}

Feders Prairie Seed Co.

12871380 th Ave.

Blue Earth. MN

56013

PHONE: (507) 526-3049

$\begin{array}{ll}\square \text { TREE } & \square \text { LOCAL TREE } \\ \square \text { SHRUB } & \square \text { LOCAL SHRUB } \\ \nabla \text { GRASS } & \square \text { LOCAL GRASS } \\ \nabla \text { FORBS } & \square \text { LOCAL FORBS } \\ \square \text { WETLAND } & \square \text { LOCAL WETLAND }\end{array}$

URL: none currently available

Bovey

Spangle Creek Labs

21950 CR 445

Bovey, MN

55709

PHONE: (218) 247-0245

$\begin{array}{ll}\square \text { TREE } & \square \text { LOCAL TREE } \\ \square \text { SHRUB } & \square \text { LOCAL SHRUB } \\ \square \text { GRASS } & \square \text { LOCAL GRASS } \\ \square \text { FORBS } & \square \text { LOCAL FORBS } \\ \square \text { WETLAND } & \square \text { LOCAL WETLAND }\end{array}$

URL: none currently available

\section{Chanhassen}

Wilsons Northwest Nursery Wholesale

9150 Great Plains Blvd.

Chanhassen, MN

55317

PHONE: (952) 445-4088

$\begin{array}{ll}\square \text { TREE } & \square \text { LOCAL TREE } \\ \square \text { SHRUB } & \square \text { LOCAL SHRUB } \\ \square \text { GRASS } & \square \text { LOCAL GRASS } \\ \square \text { FORBS } & \square \text { LOCAL FORBS } \\ \square \text { WETLAND } & \square \text { LOCAL WETLAND }\end{array}$

URL: none currently available 


\section{Cottonwood}

Mohn Seed $\mathrm{C}_{0}$

RR1, Box 152"

Cottonwood. MN

56229

PHONE: (507) 423-6482

URL: none currently available

\section{Dunas}

Werner Farm Seeds

3104 Millersburg Blvd

Dunas, MN

55019

PHONE: (507) 645-7995

\begin{tabular}{|c|c|}
\hline$\square$ TREE & $\square$ LOCAL TREE \\
\hline$\square$ SHRUB & $\square$ LOCAL SHRUB \\
\hline$\checkmark$ GRASS & $\square$ LOCAL GRASS \\
\hline$\square$ FORBS & $\square$ LOCAL FORBS \\
\hline$\square$ WETLAND & $\square$ LOCAL WETLAND \\
\hline
\end{tabular}

URL: none currently available

Edina

Twin City Seed Co

7265 Washington Ave

Edina, MN

55439

PHONE: (952) 944-7105

URL: mww.twincityseed.com

$\begin{array}{ll}\square \text { TREE } & \square \text { LOCAL TREE } \\ \square \text { SHRUB } & \square \text { LOCAL SHRUB } \\ \square \text { GRASS } & \square \text { LOCAL GRASS } \\ \nabla \text { FORBS } & \square \text { LOCAL FORBS } \\ \square \text { WETLAND } & \square \text { LOCAL WETLAND }\end{array}$

$\square$ WETLAND

$\begin{array}{ll} & \\ \square \text { TREE } & \square \text { LOCAL TREE } \\ \square \text { SHRUB } & \square \text { LOCAL SHRUB } \\ \square \text { GRASS } & \square \text { LOCAL GRASS } \\ \square \text { FORBS } & \square \text { LOCAL FORBS } \\ \square \text { WETLAND } & \square \text { LOCAL WETLAND }\end{array}$

Ellendale

Prairie Hill Wildflowers

8955 Lemond Rd.

Ellendale, MN

56026

PHONE: (507) 451-7791

$\begin{array}{ll}\square \text { TREE } & \square \text { LOCAL TREE } \\ \square \text { SHRUB } & \square \text { LOCAL SHRUB } \\ \square \text { GRASS } & \square \text { LOCAL GRASS } \\ \nabla \text { FORBS } & \square \text { LOCAL FORBS } \\ \square \text { WETLAND } & \square \text { LOCAL WETLAND }\end{array}$

URL: none currently available

Faribault

Farmers Seed \& Nursery $C_{0}$.

818 N.W. 4th St.

Faribault, MN

55021

PHONE: (507) 334-1623

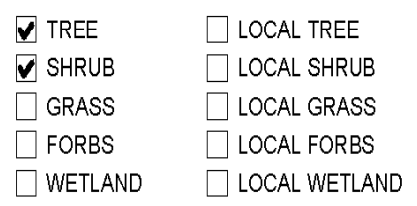

URL: www.farmerseed.com

\section{Farmington}

Bachmans Nursery Wholesale Center 235th St. W.

Farmington, MN

55024

PHONE: (651) 463-3288

$\begin{array}{ll}\nabla \text { TREE } & \square \text { LOCAL TREE } \\ \nabla \text { SHRUB } & \square \text { LOCAL SHRUB } \\ \square \text { GRASS } & \square \text { LOCAL GRASS } \\ \square \text { FORBS } & \square \text { LOCAL FORBS } \\ \square \text { WETLAND } & \square \text { LOCAL WETLAND }\end{array}$

URL: www.bachmanswholesale.com 
Fertile

Bergeson Nursery

Rt.1, Box 184"

Fertile, MN

56540

PHONE: (218) 945-6988

URL: none currently available

Kaste Seed Inc.

RR2 Box 153

Fertile, MN

56540

PHONE: (218) 945-6738

$\begin{array}{ll}\nabla \text { TREE } & \square \text { LOCAL TREE } \\ \square \text { SHRUB } & \square \text { LOCAL SHRUB } \\ \square \text { GRASS } & \square \text { LOCAL GRASS } \\ \square \text { FORBS } & \square \text { LOCAL FORBS } \\ \square \text { WETLAND } & \square \text { LOCAL WETLAND }\end{array}$

URL: none currently available

Mark Gullickson

Ri2, Box $150 \mathrm{~A}$

Fertile, MN

56540

PHONE: (218) 945-6894

$\begin{array}{ll}\square \text { TREE } & \square \text { LOCAL TREE } \\ \square \text { SHRUB } & \square \text { LOCAL SHRUB } \\ \nabla \text { GRASS } & \square \text { LOCAL GRASS } \\ \square \text { FORBS } & \square \text { LOCAL FORBS } \\ \square \text { WETLAND } & \square \text { LOCAL WETLAND }\end{array}$

$\begin{array}{ll}\square \text { TREE } & \square \text { LOCAL TREE } \\ \square \text { SHRUB } & \square \text { LOCAL SHRUB } \\ \nabla \text { GRASS } & \square \text { LOCAL GRASS } \\ \nabla \text { FORBS } & \square \text { LOCAL FORBS } \\ \square \text { WETLAND } & \square \text { LOCAL WETLAND }\end{array}$

URL: none currently available

\section{Heron Lake}

Schumachers Nursery \& Berny Farm, Inc.

37806 910th Street

Heron Lake, MN

56137

PHONE: (507) 793-2288

$\begin{array}{ll}\nabla \text { TREE } & \square \text { LOCAL TREE } \\ \nabla \text { SHRUB } & \square \text { LOCAL SHRUB } \\ \square \text { GRASS } & \square \text { LOCAL GRASS } \\ \square \text { FORBS } & \square \text { LOCAL FORBS } \\ \square \text { WETLAND } & \square \text { LOCAL WETLAND }\end{array}$

URL: none currently available

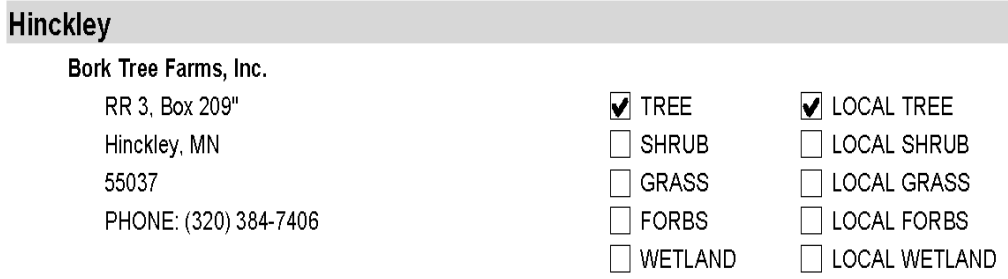

URL: www.borktreefarms.com

\section{Lakeville}

Cross Nurseries, Inc.

19774 Kenwood Trail W.

Lakeville, MN

55044

PHONE: (612) 469-2414

$\begin{array}{ll}\nabla \text { TREE } & \square \text { LOCAL TREE } \\ \nabla \text { SHRUB } & \nabla \text { LOCAL SHRUB } \\ \nabla \text { GRASS } & \square \text { LOCAL GRASS } \\ \square \text { FORBS } & \square \text { LOCAL FORBS } \\ \square \text { WETLAND } & \square \text { LOCAL WETLAND }\end{array}$

URL: www.crossnurseries.com 


\section{Litchfield}

$C \& K$ Seeds

612 S. Swift Ave

Litchfield, MN

55355

PHONE: (320) 693-8791

$\begin{array}{ll}\square \text { TREE } & \square \text { LOCAL TREE } \\ \square \text { SHRUB } & \square \text { LOCAL SHRUB } \\ \square \text { GRASS } & \square \text { LOCAL GRASS } \\ \square \text { FORBS } & \square \text { LOCAL FORBS } \\ \square \text { WETLAND } & \square \text { LOCAL WETLAND }\end{array}$

URL: none currently available

\section{Owatonna}

Wildlife Habitat

5114 N.E. 46th St.

Owatonna, MN

55060

PHONE: (507) 451-6771

$\begin{array}{ll}\square \text { TREE } & \square \text { LOCAL TREE } \\ \square \text { SHRUB } & \square \text { LOCAL SHRUB } \\ \square \text { GRASS } & \square \text { LOCAL GRASS } \\ \square \text { FORBS } & \square \text { LOCAL FORBS } \\ \square \text { WETLAND } & \square \text { LOCAL WETLAND }\end{array}$

URL: none currently available

Pine River

Sunshine Gardens

$\begin{array}{lll}\text { 1286 Shadywood Shores Dr, NW } & \square \text { TREE } & \square \text { LOCAL TREE } \\ \text { Pine River, MN } & \square \text { SHRUB } & \square \text { LOCAL SHRUB } \\ 56474 & \square \text { GRASS } & \square \text { LOCAL GRASS } \\ \text { PHONE: (218) 947-3154 } & \square \text { FORBS } & \square \text { LOCAL FORBS } \\ & \square \text { WETLAND } & \square \text { LOCAL WETLAND }\end{array}$

URL: www.sunshinegardens.tripod.com

\section{Princeton}

Prairie Restorations

P.O. Box 327

Princeton, MN

55371

PHONE: (763) 389-4342

$\begin{array}{ll}\nabla \text { TREE } & \checkmark \text { LOCAL TREE } \\ \nabla \text { SHRUB } & \checkmark \text { LOCAL SHRUB } \\ \nabla \text { GRASS } & \checkmark \text { LOCAL GRASS } \\ \nabla \text { FORBS } & \nabla \text { LOCAL FORBS } \\ \nabla \text { WETLAND } & \nabla \text { LOCAL WETLAND }\end{array}$

URL: www.prairieresto.com

\section{S. Hastings}

Out Back Nursery

15280110 th St.

S. Hastings, MN

55113-655

PHONE: (651) 438-2771

URL: www.outbacknursery.com

\section{Shakopee}

Minnesota Valley Wholesale, Inc. 14505 Johnson Memorial Dr.

Shakopee, MN

55379

PHONE: (952) 445-7120

$\begin{array}{ll}\square \text { TREE } & \square \text { LOCAL TREE } \\ \square \text { SHRUB } & \square \text { LOCAL SHRUB } \\ \square \text { GRASS } & \square \text { LOCAL GRASS } \\ \square \text { FORBS } & \square \text { LOCAL FORBS } \\ \square \text { WETLAND } & \square \text { LOCAL WETLAND }\end{array}$

$\begin{array}{ll}\nabla \text { TREE } & \square \text { LOCAL TREE } \\ \nabla \text { SHRUB } & \square \text { LOCAL SHRUB } \\ \square \text { GRASS } & \square \text { LOCAL GRASS } \\ \square \text { FORBS } & \square \text { LOCAL FORBS } \\ \square \text { WETLAND } & \square \text { LOCAL WETLAND }\end{array}$

URL: www.minnesotavalley.com 
Spring Grove

Shooting Star Native Seeds

RR 2, Box 191"

Spring Grove, MN

55974

PHONE: (507) 498-3944

$\begin{array}{ll}\square \text { TREE } & \square \text { LOCAL TREE } \\ \square \text { SHRUB } & \square \text { LOCAL SHRUB } \\ \nabla \text { GRASS } & \nabla \text { LOCAL GRASS } \\ \square \text { FORBS } & \boldsymbol{\nabla} \text { LOCAL FORBS } \\ \square \text { WETLAND } & \nabla \text { LOCAL WETLAND }\end{array}$

URL: ww.shootingstarnativeseed.com

St. Paul

Landscape Alternatives, Inc.

1705 St. Albans St.

St. Paul, MN

55113-655

PHONE: (651) 488-3142

$\begin{array}{ll}\square \text { TREE } & \square \text { LOCAL TREE } \\ \square \text { SHRUB } & \square \text { LOCAL SHRUB } \\ \square \text { GRASS } & \square \text { LOCAL GRASS } \\ \square \text { FORBS } & \square \text { LOCAL FORBS } \\ \square \text { WETLAND } & \square \text { LOCAL WETLAND }\end{array}$

URL: none currently available

Waseca

Shady Oaks Nursery

1101 N State St

Waseca, MN

56093

PHONE: (507) 835-5033

$\begin{array}{ll}\square \text { TREE } & \square \text { LOCAL TREE } \\ \square \text { SHRUB } & \square \text { LOCAL SHRUB } \\ \nabla \text { GRASS } & \square \text { LOCAL GRASS } \\ \square \text { FORBS } & \square \text { LOCAL FORBS } \\ \square \text { WETLAND } & \square \text { LOCAL WETLAND }\end{array}$

URL: www.shadyoaks.com

Willow River

Minnesota DNR General Andrews Nursery

PO Box 95

Willow River, MN

55795-009

PHONE: (218) 372-3183

$\begin{array}{ll}\square \text { TREE } & \square \text { LOCAL TREE } \\ \square \text { SHRUB } & \square \text { LOCAL SHRUB } \\ \square \text { GRASS } & \square \text { LOCAL GRASS } \\ \square \text { FORBS } & \square \text { LOCAL FORBS } \\ \square \text { WETLAND } & \square \text { LOCAL WETLAND }\end{array}$

URL: hitp://Www.dnr.state.mn.usfforestry/nurseries/index.html

Windom

Salix Ecological Resources

PO Box 337

Windom, MN

56101

PHONE: (507) 831-5729

$\begin{array}{ll}\square \text { TREE } & \square \text { LOCAL TREE } \\ \square \text { SHRUB } & \square \text { LOCAL SHRUB } \\ \square \text { GRASS } & \square \text { LOCAL GRASS } \\ \square \text { FORBS } & \square \text { LOCAL FORBS } \\ \square \text { WETLAND } & \square \text { LOCAL WETLAND }\end{array}$

URL: none currently available

\begin{tabular}{|c|c|c|}
\hline Winona & & \\
\hline Prairie Moon Nursery & & \\
\hline 31837 Bur Oak Lane & $\checkmark$ TREE & $\checkmark$ LOCAL TREE \\
\hline Winona, MN & $\nabla$ SHRUB & $\checkmark$ LOCAL SHRUB \\
\hline 55987 & $\checkmark$ GRASS & $\nabla$ LOCAL GRASS \\
\hline PHONE: (507) 452-1362 & $\checkmark$ FORBS & $\underline{\nabla}$ LOCAL FORBS \\
\hline & $\checkmark$ WETLAND & $\checkmark$ LOCAL WETLAND \\
\hline
\end{tabular}

URL: www.prairiemoon.com 


\section{Missouri}

Auxvasse

Prairie Hill Farm

877 CR 263

Auxvasse, MO

65231

PHONE: (573) 387-4680

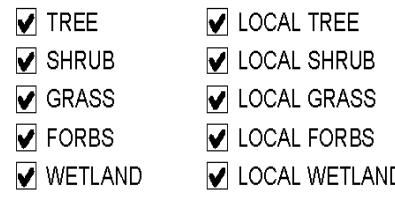

URL: none currently available

\section{Chillicothe}

Reeds Seeds, Inc.

PO Box 230

Chillicothe, MO

64601

PHONE: (660) 646-4426

$\begin{array}{ll}\square \text { TREE } & \square \text { LOCAL TREE } \\ \square \text { SHRUB } & \square \text { LOCAL SHRUB } \\ \square \text { GRASS } & \square \text { LOCAL GRASS } \\ \square \text { FORBS } & \square \text { LOCAL FORBS } \\ \square \text { WETLAND } & \square \text { LOCAL WETLAND }\end{array}$

URL: www.reedseeds.com

\section{Clarksville}

Bowood Farms

R.R. 1, Box 90

Clarksville, MO

63336

PHONE: (800) 246-3840

$\begin{array}{ll}\square \text { TREE } & \square \text { LOCAL TREE } \\ \nabla \text { SHRUB } & \square \text { LOCAL SHRUB } \\ \nabla \text { GRASS } & \square \text { LOCAL GRASS } \\ \nabla \text { FORBS } & \square \text { LOCAL FORBS } \\ \square \text { WETLAND } & \square \text { LOCAL WETLAND }\end{array}$

URL: none currently available

\section{Clifton Hill}

Shepherd Farms

Rt. 1 Box 7

Cliton Hill, MO

65244

$\square$ TREE

$\square$ SHRUB

$\square$ GRASS

$\square$ LOCAL TREE

$\begin{array}{ll}\square \text { FORBS } & \square \text { LOCAL FORBS } \\ \square \text { WETLAND } & \square \text { LOCAL WETLAND }\end{array}$

URL: www.shepherdfarms.com

Clinton

Sharp Bros. Seed

396 SW Davis-Ladue

Clinton, MO

64735

PHONE: (660) 885-7551

$\begin{array}{ll}\square \text { TREE } & \square \text { LOCAL TREE } \\ \square \text { SHRUB } & \square \text { LOCAL SHRUB } \\ \square \text { GRASS } & \square \text { LOCAL GRASS } \\ \square \text { FORBS } & \square \text { LOCAL FORBS } \\ \square \text { WETLAND } & \square \text { LOCAL WETLAND }\end{array}$

URL: www.sharpseed.com

\section{Doniphan}

Ripley County Farms

PO Box 614

Doniphan, MO

63935

PHONE: (573) 996-3449

$\begin{array}{ll}\square \text { TREE } & \square \text { LOCAL TREE } \\ \square \text { SHRUB } & \square \text { LOCAL SHRUB } \\ \square \text { GRASS } & \square \text { LOCAL GRASS } \\ \square \text { FORBS } & \square \text { LOCAL FORBS } \\ \square \text { WETLAND } & \square \text { LOCAL WETLAND }\end{array}$

URL: none currently available 


\section{Elk Creek}

Hamilton Seed \& Wildflower

16786 Brown Rd.

Elk Creek, MO

65464

PHONE: (417) 967-2190

URL: www.hamiltonseed.com

\section{Elsberry}

Forrest Keeling Nursery

Box 135

$\nabla$ TREE $\quad \nabla$ LOCAL TREE

Elsberry, MO

63343

PHONE: (573) 898-5571

$\checkmark$ SHRUB $\quad \nabla$ LOCAL SHRUB

$\square$ GRASS $\square$ LOCAL GRASS

$\checkmark$ FORBS $\square$ LOCAL FORBS

$\nabla$ WETLAND $\square$ LOCAL WETLAND

URL: www.fknursery.com

Lovelace Seeds, Inc.

1187 Brownsmill Rd

Elsberry, MO

63343

PHONE: (573) 898-2103

$\begin{array}{ll}\nabla \text { TREE } & \square \text { LOCAL TREE } \\ \nabla \text { SHRUB } & \square \text { LOCAL SHRUB } \\ \square \text { GRASS } & \square \text { LOCAL GRASS } \\ \square \text { FORBS } & \square \text { LOCAL FORBS } \\ \nabla \text { WETLAND } & \square \text { LOCAL WETLAND }\end{array}$

URL: http://Www inweb/ lovelace

Plant Materials Center

2803 N. Hwy 79

Elsberry, MO

63343

PHONE: (573) 898-2012

$\begin{array}{ll}\square \text { TREE } & \square \text { LOCAL TREE } \\ \square \text { SHRUB } & \square \text { LOCAL SHRUB } \\ \nabla \text { GRASS } & \square \text { LOCAL GRASS } \\ \square \text { FORBS } & \square \text { LOCAL FORBS } \\ \square \text { WETLAND } & \square \text { LOCAL WETLAND }\end{array}$

JRL: http://plant-materials.nrcs.usda.gov/mopmc/

\section{Gallatin}

$\mathrm{J} \& \mathrm{~J}$ Seed Co.

PO Box 341

Gallatin, MO

64640

PHONE: (660) 663-3165

$\begin{array}{ll}\square \text { TREE } & \square \text { LOCAL TREE } \\ \square \text { SHRUB } & \square \text { LOCAL SHRUB } \\ \square \text { GRASS } & \square \text { LOCAL GRASS } \\ \square \text { FORBS } & \square \text { LOCAL FORBS } \\ \square \text { WETLAND } & \square \text { LOCAL WETLAND }\end{array}$

$\begin{array}{ll}\square \text { TREE } & \square \text { LOCAL TREE } \\ \square \text { SHRUB } & \square \text { LOCAL SHRUB } \\ \square \text { GRASS } & \square \text { LOCAL GRASS } \\ \square \text { FORBS } & \square \text { LOCAL FORBS } \\ \square \text { WETLAND } & \square \text { LOCAL WETLAND }\end{array}$

URL: www.jandiseed.com

Glencoe

Gilberg Perennial Farms

2906 Ossenfort Rd.

Glencoe, MO

63038

PHONE: (314) 458-2033

$\begin{array}{ll}\square \text { TREE } & \square \text { LOCAL TREE } \\ \square \text { SHRUB } & \square \text { LOCAL SHRUB } \\ \square \text { GRASS } & \square \text { LOCAL GRASS } \\ \square \text { FORBS } & \square \text { LOCAL FORBS } \\ \square \text { WETLAND } & \square \text { LOCAL WETLAND }\end{array}$

URL: none currently available 
Holt

Arborvillage Farm Nursery

P.O. Box 227

Holt, MO

64048

PHONE: (816) 264-3911

$\begin{array}{ll}\nabla \text { TREE } & \square \text { LOCAL TREE } \\ \square \text { SHRUB } & \square \text { LOCAL SHRUB } \\ \square \text { GRASS } & \square \text { LOCAL GRASS } \\ \square \text { FORBS } & \square \text { LOCAL FORBS } \\ \square \text { WETLAND } & \square \text { LOCAL WETLAND }\end{array}$

URL: www.arborvillagellc.com

Jefferson City

Missouri Wildflowers Nursery

9814 Pleasant Hill Rd

$\nabla$ TREE $\quad \nabla$ LOCAL TREE

Jefferson City, MO

65109

$\checkmark$ SHRUB $\quad \nabla$ LOCAL SHRUB

PHONE: (314) 496-3492

$\nabla$ GRASS $\nabla$ LOCAL GRASS

$\nabla$ FORBS $\quad \nabla$ LOCAL FORBS

$\square$ WETLAND $\square$ LOCAL WETLAND

URL: www.mowildflowers.net/

Tri-Star Seed Co.

1011 W. Miller St.

Jefferson City, MO

65109

PHONE: (573) 636-4720

$\begin{array}{ll}\square \text { TREE } & \square \text { LOCAL TREE } \\ \square \text { SHRUB } & \square \text { LOCAL SHRUB } \\ \square \text { GRASS } & \square \text { LOCAL GRASS } \\ \square \text { FORBS } & \square \text { LOCAL FORBS } \\ \square \text { WETLAND } & \square \text { LOCAL WETLAND }\end{array}$

URL: none currently available

\section{Kingsville}

Flick Bros. Seed

1781 N.W. 50th Rd.

Kingsville, MO

64061

PHONE: (816) 597-3822

$\begin{array}{ll}\square \text { TREE } & \square \text { LOCAL TREE } \\ \square \text { SHRUB } & \square \text { LOCAL SHRUB } \\ \square \text { GRASS } & \square \text { LOCAL GRASS } \\ \square \text { FORBS } & \square \text { LOCAL FORBS } \\ \square \text { WETLAND } & \square \text { LOCAL WETLAND }\end{array}$

URL: www.seedguys.com

\begin{tabular}{|c|c|c|}
\hline \multicolumn{3}{|l|}{ Licking } \\
\hline \multicolumn{3}{|l|}{ MO State Forest Nursery } \\
\hline 14027 Shafer Road & $\nabla$ TREE & $\checkmark$ LOCAL TREE \\
\hline Licking, MO & $\checkmark$ SHRUB & $\checkmark$ LOCAL SHRUB \\
\hline 65542 & $\square$ GRASS & $\square$ LOCAL GRASS \\
\hline PHONE: (573) 674-3229 & $\square$ FORBS & $\square$ LOCAL FORBS \\
\hline & $\checkmark$ WETLAND & $\checkmark$ LOCAL WETLAND \\
\hline
\end{tabular}

URL: www.conservation.state.mo.us

\section{Louisiana}

\section{Stark Bros.}

P.O.Box 10

Louisiana, MO

63353

PHONE: (800) 325-4180

$\begin{array}{ll}\nabla \text { TREE } & \square \text { LOCAL TREE } \\ \nabla \text { SHRUB } & \square \text { LOCAL SHRUB } \\ \square \text { GRASS } & \square \text { LOCAL GRASS } \\ \square \text { FORBS } & \square \text { LOCAL FORBS } \\ \square \text { WETLAND } & \square \text { LOCAL WETLAND }\end{array}$

URL: wWw.starkbros.com 
Macon

Allied Seed

1108 Hilldale Dr.

Macon, MO

63552

PHONE: (660) 385-6690

$\begin{array}{ll}\square \text { TREE } & \square \text { LOCAL TREE } \\ \square \text { SHRUB } & \square \text { LOCAL SHRUB } \\ \square \text { GRASS } & \square \text { LOCAL GRASS } \\ \square \text { FORBS } & \square \text { LOCAL FORBS } \\ \square \text { WETLAND } & \square \text { LOCAL WETLAND }\end{array}$

URL: www.alliedseed.com

\section{New Madrid}

Heartland Nursery $\mathrm{C}_{0}$.

311 Main St

New Madrid, MO

63869

PHONE: (573) 748-5515

$\begin{array}{ll}\nabla \text { TREE } & \square \text { LOCAL TREE } \\ \square \text { SHRUB } & \square \text { LOCAL SHRUB } \\ \square \text { GRASS } & \square \text { LOCAL GRASS } \\ \square \text { FORBS } & \square \text { LOCAL FORBS } \\ \square \text { WETLAND } & \square \text { LOCAL WETLAND }\end{array}$

URL: none currently available

Rolla

Missouri Southern Seed Corp.

2050 Old Saint James Ro.

Rolla, MO

65401

$\begin{array}{ll}\square \text { TREE } & \square \text { LOCAL TREE } \\ \square \text { SHRUB } & \square \text { LOCAL SHRUB } \\ \nabla \text { GRASS } & \square \text { LOCAL GRASS } \\ \square \text { FORBS } & \square \text { LOCAL FORBS } \\ \square \text { WETLAND } & \square \text { LOCAL WETLAND }\end{array}$

PHONE: (573) 364-1336

$\square$ WETLAND $\square$ LOCAL WETLAND

URL: none currently available

Winston

Sur-Gro Plant Food Inc.

RR 1

Winston, MO

64689

PHONE: (816) 632-7214

$\begin{array}{ll}\square \text { TREE } & \square \text { LOCAL TREE } \\ \square \text { SHRUB } & \square \text { LOCAL SHRUB } \\ \square \text { GRASS } & \square \text { LOCAL GRASS } \\ \square \text { FORBS } & \square \text { LOCAL FORBS } \\ \square \text { WETLAND } & \square \text { LOCAL WETLAND }\end{array}$

URL: none currently available

\section{Mississippi}

\section{Cleveland}

Jimmy Sanders Seed Co.

P.O. Box 1169

$\begin{array}{ll}\square \text { TREE } & \square \text { LOCAL TREE } \\ \square \text { SHRUB } & \square \text { LOCAL SHRUB } \\ \square \text { GRASS } & \square \text { LOCAL GRASS } \\ \square \text { FORBS } & \square \text { LOCAL FORBS } \\ \square \text { WETLAND } & \square \text { LOCAL WETLAND }\end{array}$

Cleveland, MS

38732

PHONE: (601) 843-3626

$\square$ WETLAND $\square$ LOCAL WETLAND

URL: www.jssanders.com

\section{Coffeeville}

Plant Materials Center 2533 County Rd. 65

Coffeeville, MS

38922

PHONE: (662) 675-2588

$\begin{array}{ll}\square \text { TREE } & \square \text { LOCAL TREE } \\ \square \text { SHRUB } & \square \text { LOCAL SHRUB } \\ \square \text { GRASS } & \square \text { LOCAL GRASS } \\ \square \text { FORBS } & \square \text { LOCAL FORBS } \\ \square \text { WETLAND } & \square \text { LOCAL WETLAND }\end{array}$

URL: http://plant-materials.nrcs.usda.gov/mspmc/ 
Jackson

Mississippi Forestry Commission

301 N Lamar Suite 300

Jackson, MS

39201

PHONE: (601) 359-1386

URL: http://WwW.mfc.state.ms.us/

\section{Pass Christian}

Raintree Center Aquatic Gardens

119 E. 2nd St.

Pass Christian, MS

39571

PHONE: (228) 452-3137

URL: none currently available

\section{Perkinston}

Green Forest Nursery

1478 Old Hwy. 26

Perkinston, MS

39573

PHONE: (601) 928-7266

\begin{tabular}{|c|c|}
\hline$\nabla$ TREE & $\nabla$ LOCAL TREE \\
\hline$\checkmark$ SHRUB & $\square$ LOCAL SHRUB \\
\hline$v$ GRASS & $\square$ LOCAL GRASS \\
\hline$\checkmark$ FORBS & $\square$ LOCAL FORBS \\
\hline$\nabla$ WETLAND & $\nabla$ LOCAL WETLAND \\
\hline
\end{tabular}

$\begin{array}{ll}\nabla \text { TREE } & \square \text { LOCAL TREE } \\ \square \text { SHRUB } & \square \text { LOCAL SHRUB } \\ \square \text { GRASS } & \square \text { LOCAL GRASS } \\ \square \text { FORBS } & \square \text { LOCAL FORBS } \\ \square \text { WETLAND } & \square \text { LOCAL WETLAND }\end{array}$

(2)


Belgrade

Gallatin Farmers $\mathrm{C}_{0}$.

114 Northern Pacific

Belgrade, MT

59714

PHONE: (406) 388-6242

URL: none currently available

Tree Factory

4285 Nelson Rd.

Belgrade, MT

59714

PHONE: (406) 388-1338

$\begin{array}{ll}\square \text { TREE } & \square \text { LOCAL TREE } \\ \square \text { SHRUB } & \square \text { LOCAL SHRUB } \\ \square \text { GRASS } & \square \text { LOCAL GRASS } \\ \square \text { FORBS } & \square \text { LOCAL FORBS } \\ \square \text { WETLAND } & \square \text { LOCAL WETLAND }\end{array}$

URL: none currently available

United Agri Products

100 S. Broadway

Belgrade, MT

59714

PHONE: (406) 388-4232

$\begin{array}{ll}\nabla \text { TREE } & \square \text { LOCAL TREE } \\ \square \text { SHRUB } & \square \text { LOCAL SHRUB } \\ \square \text { GRASS } & \square \text { LOCAL GRASS } \\ \square \text { FORBS } & \square \text { LOCAL FORBS } \\ \square \text { WETLAND } & \square \text { LOCAL WETLAND }\end{array}$

$\begin{array}{ll}\square \text { TREE } & \square \text { LOCAL TREE } \\ \square \text { SHRUB } & \square \text { LOCAL SHRUB } \\ \nabla \text { GRASS } & \square \text { LOCAL GRASS } \\ \nabla \text { FORBS } & \square \text { LOCAL FORBS } \\ \square \text { WETLAND } & \square \text { LOCAL WETLAND }\end{array}$

URL: none currently available

\section{Big Sandy}

Golden Harvest

Route 652

Big Sandy. MT

59520

PHONE: (406) 378-2112

$\begin{array}{ll}\square \text { TREE } & \square \text { LOCAL TREE } \\ \square \text { SHRUB } & \square \text { LOCAL SHRUB } \\ \square \text { GRASS } & \square \text { LOCAL GRASS } \\ \square \text { FORBS } & \square \text { LOCAL FORBS } \\ \square \text { WETLAND } & \square \text { LOCAL WETLAND }\end{array}$

URL: none currently available

\section{Big Timber}

Blake Nursery

316 Otter Creek Rd.

Big Timber, MT

59011

PHONE: (406) 932-4195

$\begin{array}{ll}\nabla \text { TREE } & \nabla \text { LOCAL TREE } \\ \nabla \text { SHRUB } & \nabla \text { LOCAL SHRUB } \\ \nabla \text { GRASS } & \nabla \text { LOCAL GRASS } \\ \nabla \text { FORBS } & \square \text { LOCAL FORBS } \\ \square \text { WETLAND } & \square \text { LOCAL WETLAND }\end{array}$

URL: www.blakenursery.com

\section{K-W Feed \& Grain}

127 E. Yellowstone Ave.

Big Timber, MT

59011

PHONE: (406) 932-5132

$\begin{array}{ll}\square \text { TREE } & \square \text { LOCAL TREE } \\ \square \text { SHRUB } & \square \text { LOCAL SHRUB } \\ \nabla \text { GRASS } & \square \text { LOCAL GRASS } \\ \square \text { FORBS } & \square \text { LOCAL FORBS } \\ \square \text { WETLAND } & \square \text { LOCAL WETLAND }\end{array}$

URL: none currently available 


\section{Billings}

Billings Nursery \& Landscaping

7900 S. Frontage Rd.

Billings, MT

59101

PHONE: (406) 656-2410

URL: none currently available

Bowman Grain \& Seed

1400 Minnesota Ave.

Billings, MT

59101

PHONE: (406) 252-8012

URL: none currently available

Field of Dreams Tree Farm

5134 Hazelnut Ave

Billings, MT

59106

PHONE: (406) 655-4507

$\begin{array}{ll}\square \text { TREE } & \square \text { LOCAL TREE } \\ \square \text { SHRUB } & \square \text { LOCAL SHRUB } \\ \nabla \text { GRASS } & \square \text { LOCAL GRASS } \\ \square \text { FORBS } & \square \text { LOCAL FORBS } \\ \square \text { WETLAND } & \square \text { LOCAL WETLAND }\end{array}$

URL: none currently available

Northland Seed Co.

418 Albert St

Billings, MT

59101

PHONE: (406) 252-0568

$\begin{array}{ll}\square \text { TREE } & \square \text { LOCAL TREE } \\ \square \text { SHRUB } & \square \text { LOCAL SHRUB } \\ \square \text { GRASS } & \square \text { LOCAL GRASS } \\ \square \text { FORBS } & \square \text { LOCAL FORBS } \\ \square \text { WETLAND } & \square \text { LOCAL WETLAND }\end{array}$

URL: none currently available

\section{Bozeman}

Bridger Canyon Tree Farm 8860 Bridger Canyon Road

Bozeman. MT

59715

PHONE: (406) 587-4553

$\begin{array}{ll}\nabla \text { TREE } & \nabla \text { LOCAL TREE } \\ \nabla \text { SHRUB } & \nabla \text { LOCAL SHRUB } \\ \nabla \text { GRASS } & \nabla \text { LOCAL GRASS } \\ \nabla \text { FORBS } & \square \text { LOCAL FORBS } \\ \square \text { WETLAND } & \square \text { LOCAL WETLAND }\end{array}$

URL: none currently available

Cashman Nursery
P.O. Box 10242
Bozeman, MT
59719
PHONE: (406) 587-3406
URL: none currently available
Cornerstone Landscape \& Gardens
81811 Gallatin Rd
Bozeman, MT
59718
PHONE: (406) 920-0231

$\begin{array}{ll}\square \text { TREE } & \square \text { LOCAL TREE } \\ \square \text { SHRUB } & \square \text { LOCAL SHRUB } \\ \square \text { GRASS } & \square \text { LOCAL GRASS } \\ \square \text { FORBS } & \square \text { LOCAL FORBS } \\ \square \text { WETLAND } & \square \text { LOCAL WETLAND }\end{array}$

$\begin{array}{ll}\square \text { TREE } & \square \text { LOCAL TREE } \\ \square \text { SHRUB } & \square \text { LOCAL SHRUB } \\ \square \text { GRASS } & \square \text { LOCAL GRASS } \\ \square \text { FORBS } & \square \text { LOCAL FORBS } \\ \square \text { WETLAND } & \square \text { LOCAL WETLAND }\end{array}$

URL: none currently available

$\begin{array}{ll}\square \text { TREE } & \square \text { LOCAL TREE } \\ \square \text { SHRUB } & \square \text { LOCAL SHRUB } \\ \square \text { GRASS } & \square \text { LOCAL GRASS } \\ \square \text { FORBS } & \square \text { LOCAL FORBS } \\ \square \text { WETLAND } & \square \text { LOCAL WETLAND }\end{array}$

$\begin{array}{ll}\nabla \text { TREE } & \square \text { LOCAL TREE } \\ \nabla \text { SHRUB } & \nabla \text { LOCAL SHRUB } \\ \square \text { GRASS } & \square \text { LOCAL GRASS } \\ \square \text { FORBS } & \square \text { LOCAL FORBS } \\ \square \text { WETLAND } & \square \text { LOCAL WETLAND }\end{array}$


Creekside Nursery \& Landscaping

5917 Bridger Canyon Rd

$\nabla$ TREE $\quad \nabla$ LOCAL TREE

Bozeman, MT

59715

PHONE: (406) 582-0720

$\nabla$ SHRUB $\quad \nabla$ LOCAL SHRUB

$\square$ GRASS $\square$ LOCAL GRASS

$\square$ FORBS $\square$ LOCAL FORBS

$\square$ WETLAND $\square$ LOCAL WETLAND

URL: none currently available

Shady Brook Tree Farm

PO Box 6487

Bozeman, MT

59771

PHONE: (406) 585-9756

$\begin{array}{ll}\nabla \text { TREE } & \square \text { LOCAL TREE } \\ \square \text { SHRUB } & \square \text { LOCAL SHRUB } \\ \square \text { GRASS } & \square \text { LOCAL GRASS } \\ \square \text { FORBS } & \square \text { LOCAL FORBS } \\ \square \text { WETLAND } & \square \text { LOCAL WETLAND }\end{array}$

URL: none currently available

Westscape Wholesale Nursery

4141 Springhill Road

Bozeman. MT

$\checkmark$ TREE

$\checkmark$ SHRUB

$\checkmark$ LOCAL TREE

59718

$\checkmark$ GRASS

$\checkmark$ LOCAL SHRUB

PHONE: (406) 522-3797

$\square$ FORBS $\square$ LOCAL FORBS

$\square$ WETLAND $\square$ LOCAL WETLAND

URL: none currently available

Bridger

Plant Mlaterials Center

Rt.1. Box 1189"

Bridger, MT

59014

PHONE: (406) 662-3579

$\begin{array}{ll}\square \text { TREE } & \square \text { LOCAL TREE } \\ \square \text { SHRUB } & \square \text { LOCAL SHRUB } \\ \square \text { GRASS } & \square \text { LOCAL GRASS } \\ \square \text { FORBS } & \square \text { LOCAL FORBS } \\ \square \text { WETLAND } & \square \text { LOCAL WETLAND }\end{array}$

URL: http://plant-materials.nrcs.usda.gov/mtpmc/

Butte

Beebe Grain

712 E. Front

Butte, MT

59701

PHONE: (406) 782-2763

$\begin{array}{ll}\square \text { TREE } & \square \text { LOCAL TREE } \\ \square \text { SHRUB } & \square \text { LOCAL SHRUB } \\ \square \text { GRASS } & \square \text { LOCAL GRASS } \\ \square \text { FORBS } & \square \text { LOCAL FORBS } \\ \square \text { WETLAND } & \square \text { LOCAL WETLAND }\end{array}$

URL: none currently available

Charlo

Westland Seed Inc

Box 57

Charlo, MT

59824

PHONE: (406) 644-2202

$\begin{array}{ll}\square \text { TREE } & \square \text { LOCAL TREE } \\ \square \text { SHRUB } & \square \text { LOCAL SHRUB } \\ \square \text { GRASS } & \square \text { LOCAL GRASS } \\ \square \text { FORBS } & \square \text { LOCAL FORBS } \\ \square \text { WETLAND } & \square \text { LOCAL WETLAND }\end{array}$

URL: www.westlandseed.com 
Chinook

Farmers Union Oil Co

Box 339

Chinook, MT

59523

PHONE: (406) 357-2281

$\begin{array}{ll}\square \text { TREE } & \square \text { LOCAL TREE } \\ \square \text { SHRUB } & \square \text { LOCAL SHRUB } \\ \square \text { GRASS } & \square \text { LOCAL GRASS } \\ \square \text { FORBS } & \square \text { LOCAL FORBS } \\ \square \text { WETLAND } & \square \text { LOCAL WETLAND }\end{array}$

URL: none currently available

\section{Columbia Falls}

Columbia Nursery \& Landscape

2544 9th Street West

Columbia Falls, MT

59912

PHONE: (406) 892-0339

$\begin{array}{ll}\nabla \text { TREE } & \nabla \text { LOCAL TREE } \\ \nabla \text { SHRUB } & \checkmark \text { LOCAL SHRUB } \\ \square \text { GRASS } & \square \text { LOCAL GRASS } \\ \square \text { FORBS } & \square \text { LOCAL FORBS } \\ \square \text { WETLAND } & \square \text { LOCAL WETLAND }\end{array}$

URL: none currently available

Fishers Greenhouses

1225 Mooring Road

Columbia Falls, MT

59912

PHONE: (406) 892-4475

$\begin{array}{ll}\nabla \text { TREE } & \square \text { LOCAL TREE } \\ \nabla \text { SHRUB } & \square \text { LOCAL SHRUB } \\ \square \text { GRASS } & \square \text { LOCAL GRASS } \\ \square \text { FORBS } & \square \text { LOCAL FORBS } \\ \square \text { WETLAND } & \square \text { LOCAL WETLAND }\end{array}$

URL: none currently available

LaSalle Nursery

PO Box 508

Columbia Falls, MT

59912

PHONE: (406) 892-3838

$\begin{array}{ll}\nabla \text { TREE } & \square \text { LOCAL TREE } \\ \nabla \text { SHRUB } & \square \text { LOCAL SHRUB } \\ \square \text { GRASS } & \square \text { LOCAL GRASS } \\ \square \text { FORBS } & \square \text { LOCAL FORBS } \\ \square \text { WETLAND } & \square \text { LOCAL WETLAND }\end{array}$

URL: none currently available

\section{Morning Creek Gardens}

880 Blackmer Lane

Columbia Falls, MT

59912

PHONE: (800) 250-4644

$\begin{array}{ll}\square \text { TREE } & \square \text { LOCAL TREE } \\ \square \text { SHRUB } & \square \text { LOCAL SHRUB } \\ \nabla \text { GRASS } & \square \text { LOCAL GRASS } \\ \nabla \text { FORBS } & \square \text { LOCAL FORBS } \\ \square \text { WETLAND } & \square \text { LOCAL WETLAND }\end{array}$

URL: none currently available

\section{Conrad}

Northern Seed Service

Rt. 3

Conrad, MT

59425

PHONE: (406) 627-2327

URL: none currently available

$\begin{array}{ll}\square \text { TREE } & \square \text { LOCAL TREE } \\ \square \text { SHRUB } & \square \text { LOCAL SHRUB } \\ \square \text { GRASS } & \square \text { LOCAL GRASS } \\ \square \text { FORBS } & \square \text { LOCAL FORBS } \\ \square \text { WETLAND } & \square \text { LOCAL WETLAND }\end{array}$




\section{Corvallis}

Bitterroot Restoration Inc.

445 Quast Lane

Corvallis, MT

59828

PHONE: (406) 961-4991

URL: www.bitterrootrestoration.com

\section{Canyon View Nursery}

335 Quast Lane

Corvallis, MT

59828

PHONE: (406) 961-4648

$\begin{array}{ll}\nabla \text { TREE } & \nabla \text { LOCAL TREE } \\ \nabla \text { SHRUB } & \nabla \text { LOCAL SHRUB } \\ \nabla \text { GRASS } & \nabla \text { LOCAL GRASS } \\ \nabla \text { FORBS } & \nabla \text { LOCAL FORBS } \\ \nabla \text { WETLAND } & \nabla \text { LOCAL WETLAND }\end{array}$

URL: none currently available

Moellers Nursery

946 Eastside Highway

Corvallis, MT

59828

PHONE: (406) 961-3389

$\begin{array}{ll}\square \text { TREE } & \square \text { LOCAL TREE } \\ \square \text { SHRUB } & \square \text { LOCAL SHRUB } \\ \square \text { GRASS } & \square \text { LOCAL GRASS } \\ \square \text { FORBS } & \square \text { LOCAL FORBS } \\ \square \text { WETLAND } & \square \text { LOCAL WETLAND }\end{array}$

URL: none currently available

Decker

Adsit Farm and Ranch

Box 17

Decker, MT

59025

PHONE: (406) 757-2223

$\begin{array}{ll}\nabla \text { TREE } & \square \text { LOCAL TREE } \\ \nabla \text { SHRUB } & \square \text { LOCAL SHRUB } \\ \square \text { GRASS } & \square \text { LOCAL GRASS } \\ \square \text { FORBS } & \square \text { LOCAL FORBS } \\ \square \text { WETLAND } & \square \text { LOCAL WETLAND }\end{array}$

URL: none currently available

Denton

Barber Seed Service

RR Box 62

Denton, MT

59430

PHONE: (406) 567-2211

$\begin{array}{ll}\square \text { TREE } & \square \text { LOCAL TREE } \\ \square \text { SHRUB } & \square \text { LOCAL SHRUB } \\ \square \text { GRASS } & \square \text { LOCAL GRASS } \\ \square \text { FORBS } & \square \text { LOCAL FORBS } \\ \square \text { WETLAND } & \square \text { LOCAL WETLAND }\end{array}$

$\begin{array}{ll}\square \text { TREE } & \square \text { LOCAL TREE } \\ \square \text { SHRUB } & \square \text { LOCAL SHRUB } \\ \square \text { GRASS } & \square \text { LOCAL GRASS } \\ \square \text { FORBS } & \square \text { LOCAL FORBS } \\ \square \text { WETLAND } & \square \text { LOCAL WETLAND }\end{array}$

URL: none currently available

Dillon

Co-op Supply, Inc.

$700 \mathrm{~N}$. Montana St.

Dillon, MT

59725

PHONE: (406) 683-2308

$\begin{array}{ll}\square \text { TREE } & \square \text { LOCAL TREE } \\ \square \text { SHRUB } & \square \text { LOCAL SHRUB } \\ \square \text { GRASS } & \square \text { LOCAL GRASS } \\ \square \text { FORBS } & \square \text { LOCAL FORBS } \\ \square \text { WETLAND } & \square \text { LOCAL WETLAND }\end{array}$

URL: none currently available 
Fairfield

Treasure State Seed

Box 698

Fairfield, MT

59436

PHONE: (406) 467-2514

$\begin{array}{ll}\square \text { TREE } & \square \text { LOCAL TREE } \\ \square \text { SHRUB } & \square \text { LOCAL SHRUB } \\ \square \text { GRASS } & \square \text { LOCAL GRASS } \\ \square \text { FORBS } & \square \text { LOCAL FORBS } \\ \square \text { WETLAND } & \square \text { LOCAL WETLAND }\end{array}$

URL: none currently available

Froid

Farmers Union Oil $\mathrm{Co}_{0}$

105 2nd Ave N.

Froid, MT

59226

$\square$ SHRUB $\quad \square$ LOCAL SHRUB

PHONE: (406) 766-2339

$\square$ GRASS $\square$ LOCAL GRASS

$\nabla$ FORBS $\square$ LOCAL FORBS

$\square$ WETLAND $\square$ LOCAL WETLAND

URL: none currently available

\section{Glasgow}

Rohde Seed Co.

Box 944

Glasgow, MT

59230

PHONE: (406) 228-9581

$\square$ SHRUB $\square$ LOCAL SHRUB

$\nabla$ GRASS $\nabla$ LOCAL GRASS

$\square$ FORBS $\quad \square$ LOCAL FORBS

$\square$ WETLAND $\square$ LOCAL WETLAND

URL: none currently available

\section{Great Falls}

Cenex/Farmers Union Oil $\mathrm{C}_{0}$

Box 2483

Great Falls, MT

59403

PHONE: (406) 453-2435

$\begin{array}{ll}\square \text { TREE } & \square \text { LOCAL TREE } \\ \square \text { SHRUB } & \square \text { LOCAL SHRUB } \\ \square \text { GRASS } & \square \text { LOCAL GRASS } \\ \square \text { FORBS } & \square \text { LOCAL FORBS } \\ \square \text { WETLAND } & \square \text { LOCAL WETLAND }\end{array}$

URL: none currently available

Forde Nursery \& Landscaping

2025 2nd Avenue N.W.

Great Fâlls, MT

59404

PHONE: (406) 727-0950

$\begin{array}{ll}\nabla \text { TREE } & \square \text { LOCAL TREE } \\ \square \text { SHRUB } & \square \text { LOCAL SHRUB } \\ \square \text { GRASS } & \square \text { LOCAL GRASS } \\ \square \text { FORBS } & \square \text { LOCAL FORBS } \\ \square \text { WETLAND } & \square \text { LOCAL WETLAND }\end{array}$

URL: none currently available

\section{Hamilton}

Bitterroot Nursery

521 Eastside Highway

Hamilton, MT

59840

PHONE: (406) 961-3806

$\begin{array}{ll}\nabla \text { TREE } & \square \text { LOCAL TREE } \\ \nabla \text { SHRUB } & \square \text { LOCAL SHRUB } \\ \square \text { GRASS } & \square \text { LOCAL GRASS } \\ \square \text { FORBS } & \square \text { LOCAL FORBS } \\ \square \text { WETLAND } & \square \text { LOCAL WETLAND }\end{array}$

URL: none currently available 
Lakeland Feed \& Supply

Box 298

Hamilton, MT

59840

PHONE: (406) 363-2334

URL: none currently available

$\begin{array}{ll}\square \text { TREE } & \square \text { LOCAL TREE } \\ \square \text { SHRUB } & \square \text { LOCAL SHRUB } \\ \square \text { GRASS } & \square \text { LOCAL GRASS } \\ \square \text { FORBS } & \square \text { LOCAL FORBS } \\ \square \text { WETLAND } & \square \text { LOCAL WETLAND }\end{array}$

$\square$ WETLAND $\square$ LOCAL WETLAND

Helena

Chadwick Nursery Landscape

2010 Custer

Helena, MT

59602

PHONE: (406) 442-3931

$\checkmark$ SHRUB $\nabla$ LOCAL SHRUB

$\nabla$ GRASS $\square$ LOCAL GRASS

$\checkmark$ FORBS $\square$ LOCAL FORBS

$\square$ WETLAND $\square$ LOCAL WETLAND

URL: none currently available

Valley Nursery

Box 4845

Helena, MT

59604

PHONE: (406) 458-5919

$\checkmark$ TREE $\quad \boldsymbol{V}$ LOCAL TREE

$\checkmark$ SHRUB $\quad \forall$ LOCAL SHRUB

$\square$ GRASS $\square$ LOCAL GRASS

$\checkmark$ FORBS $\quad \nabla$ LOCAL FORBS

$\checkmark$ WETLAND $\checkmark$ LOCAL WETLAND

URL: none currently available

Heron

Circle D Farms

58 Golden Pond Rd

Heron, MT

59844

PHONE: (406) 847-2763

$\checkmark$ TREE $\quad \boldsymbol{v}$ LOCAL TREE

$\square$ SHRUB $\square$ LOCAL SHRUB

$\square$ GRASS $\square$ LOCAL GRASS

$\square$ FORBS $\square$ LOCAL FORBS

$\square$ WETLAND $\square$ LOCAL WETLAND

URL: none currently available

\section{Kalispell}

Glacier Nursery

4343 Montana Highway 35

Kalispell, MT

59901

PHONE: (406) 755-2248

$\checkmark$ TREE $\boldsymbol{\nabla}$ LOCAL TREE

$\checkmark$ SHRUB $\quad \nabla$ LOCAL SHRUB

$\square$ GRASS $\square$ LOCAL GRASS

$\square$ FORBS $\square$ LOCAL FORBS

$\square$ WETLAND $\square$ LOCAL WETLAND

URL: none currently available

P \& Ds Tree Farm

415 Sonstelie Rd

Kalispell, MT

59901

PHONE: (406) 755-2698

$\begin{array}{ll}\square \text { TREE } & \square \text { LOCAL TREE } \\ \square \text { SHRUB } & \square \text { LOCAL SHRUB } \\ \square \text { GRASS } & \square \text { LOCAL GRASS } \\ \square \text { FORBS } & \square \text { LOCAL FORBS } \\ \square \text { WETLAND } & \square \text { LOCAL WETLAND }\end{array}$

URL: none currently available 
Lewistown

Central Feed Co.

220 E. Main

Lewistown, MT

59457

PHONE: (406) 538-5451

$\begin{array}{ll}\square \text { TREE } & \square \text { LOCAL TREE } \\ \square \text { SHRUB } & \square \text { LOCAL SHRUB } \\ \square \text { GRASS } & \square \text { LOCAL GRASS } \\ \square \text { FORBS } & \square \text { LOCAL FORBS } \\ \square \text { WETLAND } & \square \text { LOCAL WETLAND }\end{array}$

URL: none currently available

Livingston

Deep Creek Garden

90 Deep Creek Road

$\nabla$ TREE $\quad \nabla$ LOCAL TREE

Livingston, MT

59047

PHONE: (406) 222-7109

$\checkmark$ SHRUB $\quad \nabla$ LOCAL SHRUB

$\checkmark$ GRASS $\square$ LOCAL GRASS

$\checkmark$ FORBS $\quad \nabla$ LOCAL FORBS

$\square$ WETLAND $\square$ LOCAL WETLAND

URL: none currently available

Native Landscapes \& Reclamation

314 South H St

Livingston, MT

59047

PHONE: (406) 222-0457

$\begin{array}{ll}\nabla \text { TREE } & \nabla \text { LOCAL TREE } \\ \square \text { SHRUB } & \square \text { LOCAL SHRUB } \\ \square \text { GRASS } & \square \text { LOCAL GRASS } \\ \square \text { FORBS } & \square \text { LOCAL FORBS } \\ \square \text { WETLAND } & \square \text { LOCAL WETLAND }\end{array}$

URL: none currently available

River Inn Nursery

4950 US Highway $89 \mathrm{~S}$

Livingston, MT

59047-917

PHONE: (406) 222-2429

$\checkmark$ SHRUB $\quad \nabla$ LOCAL SHRUB

$\nabla$ GRASS $\square$ LOCAL GRASS

$\checkmark$ FORBS $\square$ LOCAL FORBS

$\square$ WETLAND $\square$ LOCAL WETLAND

URL: none currently available

Tri-Valley Feed

Rt. 62, Box 3252"

Livingston, MT

59047

PHONE: (406) 222-1132

$\square$ SHRUB $\square$ LOCAL SHRUB

$\square$ GRASS $\square$ LOCAL GRASS

$\checkmark$ FORBS $\square$ LOCAL FORBS

$\square$ WETLAND $\square$ LOCAL WETLAND

URL: none currently available

Valley Feed

Rt. 62, Box 3252"

Livingston, MT

59047

PHONE: (406) 222-1132

$\begin{array}{ll}\square \text { TREE } & \square \text { LOCAL TREE } \\ \square \text { SHRUB } & \square \text { LOCAL SHRUB } \\ \square \text { GRASS } & \square \text { LOCAL GRASS } \\ \square \text { FORBS } & \square \text { LOCAL FORBS } \\ \square \text { WETLAND } & \square \text { LOCAL WETLAND }\end{array}$

URL: none currently available

Malta

Northern Ag. Service

HC 65, Box 5500"

Malta, MT

59538

PHONE: (406) 654-2022

$\begin{array}{ll}\square \text { TREE } & \square \text { LOCAL TREE } \\ \square \text { SHRUB } & \square \text { LOCAL SHRUB } \\ \square \text { GRASS } & \square \text { LOCAL GRASS } \\ \square \text { FORBS } & \square \text { LOCAL FORBS } \\ \square \text { WETLAND } & \square \text { LOCAL WETLAND }\end{array}$

URL: none currently available 


\section{Manhattan}

Blue Mountain Tree Farm

3500 Veltkamp Road

Manhattan, MT

59741

PHONE: (503) 644-3418

$\begin{array}{ll}\nabla \text { TREE } & \square \text { LOCAL TREE } \\ \square \text { SHRUB } & \square \text { LOCAL SHRUB } \\ \square \text { GRASS } & \square \text { LOCAL GRASS } \\ \square \text { FORBS } & \square \text { LOCAL FORBS } \\ \square \text { WETLAND } & \square \text { LOCAL WETLAND }\end{array}$

URL: none currently available

Reese Creek Tree Farm

5944 West Dry Creek Road

Manhattan, MT

59741

PHONE: (406) 284-6566

$\begin{array}{ll}\nabla \text { TREE } & \square \text { LOCAL TREE } \\ \square \text { SHRUB } & \square \text { LOCAL SHRUB } \\ \square \text { GRASS } & \square \text { LOCAL GRASS } \\ \square \text { FORBS } & \square \text { LOCAL FORBS } \\ \square \text { WETLAND } & \square \text { LOCAL WETLAND }\end{array}$

URL: none currently available

\section{Miles City}

Cenex Seed Plant

Box 956

Miles City, MT

59301

PHONE: (406) 232-4760

$\begin{array}{ll}\square \text { TREE } & \square \text { LOCAL TREE } \\ \square \text { SHRUB } & \square \text { LOCAL SHRUB } \\ \square \text { GRASS } & \square \text { LOCAL GRASS } \\ \square \text { FORBS } & \square \text { LOCAL FORBS } \\ \square \text { WETLAND } & \square \text { LOCAL WETLAND }\end{array}$

URL: none currently available

\section{Missoula}

Caras Nursery \& Landscaping

2727 S. 3rd West

Missoula, MT

59801

PHONE: (406) 543-3333

$\nabla$ TREE

$\checkmark$ SHRUB

$\checkmark$ GRASS

$\checkmark$ FORBS

$\square$ WETLAND

$\square$ LOCAL TREE

$\square$ LOCAL SHRUB

$\square$ LOCAL GRASS

$\square$ LOCAL FORBS

URL: www.carasnursery.com

Montana Division of Forestry 2705 Spurgin Road

$\checkmark$ TREE

\section{$\checkmark$ SHRUB}

$\square$ LOCAL WETLAND

Missoula, MT

59801

PHONE: (406) 542-4244

$\square$ GRASS

FORBS

$\checkmark$ WETLAND

$\checkmark$ LOCAL TREE $\nabla$ LOCAL SHRUB

$\square$ LOCAL GRASS

$\square$ LOCAL FORBS

URL: http://Www dnrc.state.mt.us/forestry/Nurseryindex.htm

\section{Single Tree Nursery}

9480 Singletree Lane

Missoula, MT

59803

PHONE: (406) 329-3287

$\begin{array}{ll}\nabla \text { TREE } & \square \text { LOCAL TREE } \\ \square \text { SHRUB } & \square \text { LOCAL SHRUB } \\ \square \text { GRASS } & \square \text { LOCAL GRASS } \\ \square \text { FORBS } & \square \text { LOCAL FORBS } \\ \square \text { WETLAND } & \square \text { LOCAL WETLAND }\end{array}$

URL: none currently available 


\section{Moccasin}

Heartland Seed Co

HC 90 Box 12

Moccasin. MT

59462

PHONE: (406) 423-5600

$\begin{array}{ll}\square \text { TREE } & \square \text { LOCAL TREE } \\ \square \text { SHRUB } & \square \text { LOCAL SHRUB } \\ \square \text { GRASS } & \square \text { LOCAL GRASS } \\ \square \text { FORBS } & \square \text { LOCAL FORBS } \\ \square \text { WETLAND } & \square \text { LOCAL WETLAND }\end{array}$

URL: none currently available

\section{N. Hamilton}

\section{Garden City Seeds}

778 Hwy. 93

N. Hamilton, MT

9840-9712

PHONE: (406) 961-4837

$\begin{array}{ll}\square \text { TREE } & \square \text { LOCAL TREE } \\ \square \text { SHRUB } & \square \text { LOCAL SHRUB } \\ \square \text { GRASS } & \square \text { LOCAL GRASS } \\ \nabla \text { FORBS } & \square \text { LOCAL FORBS } \\ \square \text { WETLAND } & \square \text { LOCAL WETLAND }\end{array}$

URL: none currently available

\section{Paradise}

Horseshoe Grove Nursery and Landscaping

PO Box 15

Paradise, MT

59856

PHONE: (406) 826-3260

$\checkmark$ SHRUB $\square$ LOCAL SHRUB

$\nabla$ GRASS $\square$ LOCAL GRASS

$\square$ FORBS $\square$ LOCAL FORBS

$\checkmark$ WETLAND $\square$ LOCAL WETLAND

URL: none currently available

\section{Plains}

Lawyer Nursery, Inc.

950 Hwy. $200 \mathrm{~W}$

Plains, MT

59859-970

PHONE: (406) 826-3881

URL: www.lawwernursery.com

Montana Native Nursery

92 Helterline Lane

Plains. MT

59859

PHONE: (406) 826-0067

Power

Cenex/Power Farmers Elev. Co.

Box 215

Power, MT

59468

PHONE: (406) 463-2281

URL: none currently available

$\begin{array}{ll}\square \text { TREE } & \square \text { LOCAL TREE } \\ \nabla \text { SHRUB } & \checkmark \text { LOCAL SHRUB } \\ \square \text { GRASS } & \square \text { LOCAL GRASS } \\ \nabla \text { FORBS } & \square \text { LOCAL FORBS } \\ \square \text { WETLAND } & \square \text { LOCAL WETLAND }\end{array}$

$\begin{array}{ll}\nabla \text { TREE } & \nabla \text { LOCAL TREE } \\ \nabla \text { SHRUB } & \nabla \text { LOCAL SHRUB } \\ \nabla \text { GRASS } & \nabla \text { LOCAL GRASS } \\ \nabla \text { FORBS } & \nabla \text { LOCAL FORBS } \\ \nabla \text { WETLAND } & \square \text { LOCAL WETLAND }\end{array}$

$\nabla$ WETLAND $\nabla$ LOCAL WETLAND

$\begin{array}{ll}\square \text { TREE } & \square \text { LOCAL TREE } \\ \square \text { SHRUB } & \square \text { LOCAL SHRUB } \\ \nabla \text { GRASS } & \square \text { LOCAL GRASS } \\ \square \text { FORBS } & \square \text { LOCAL FORBS } \\ \square \text { WETLAND } & \square \text { LOCAL WETLAND }\end{array}$


Proctor

Adams Ranch \& Trees

Box 143

Proctor, MT

59929

PHONE: (406) 849-5613

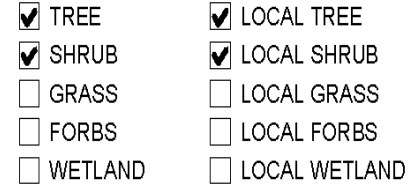

URL: none currently available

\section{Red Lodge}

Rocky Mountain Flower Farm

1409 North Broadway Ave.

Red Lodge, MT

59068

PHONE: (406) 446-3338

$\begin{array}{ll}\nabla \text { TREE } & \nabla \text { LOCAL TREE } \\ \nabla \text { SHRUB } & \nabla \text { LOCAL SHRUB } \\ \square \text { GRASS } & \square \text { LOCAL GRASS } \\ \square \text { FORBS } & \square \text { LOCAL FORBS } \\ \square \text { WETLAND } & \square \text { LOCAL WETLAND }\end{array}$

URL: none currently available

Ronan

Westland Seed, Inc.

1308 Round Butte Rd. W.

Ronan, MT

59864

PHONE: (406) 676-4100

$\begin{array}{ll}\square \text { TREE } & \square \text { LOCAL TREE } \\ \square \text { SHRUB } & \square \text { LOCAL SHRUB } \\ \square \text { GRASS } & \square \text { LOCAL GRASS } \\ \square \text { FORBS } & \square \text { LOCAL FORBS } \\ \square \text { WETLAND } & \square \text { LOCAL WETLAND }\end{array}$

URL: www.westlandseed.com

Saco

First Creek Seeds

179 Beavertown Rd.

Saco, MT

59261

PHONE: (406) 527-3334

$\begin{array}{ll}\square \text { TREE } & \square \text { LOCAL TREE } \\ \square \text { SHRUB } & \square \text { LOCAL SHRUB } \\ \square \text { GRASS } & \square \text { LOCAL GRASS } \\ \square \text { FORBS } & \square \text { LOCAL FORBS } \\ \square \text { WETLAND } & \square \text { LOCAL WETLAND }\end{array}$

URL: none currently available

Shelby

Big Sky Wholesale Seed

Box 852

Shelby, MT

59474

PHONE: (406) 434-5011

$\begin{array}{ll}\square \text { TREE } & \square \text { LOCAL TREE } \\ \square \text { SHRUB } & \square \text { LOCAL SHRUB } \\ \square \text { GRASS } & \square \text { LOCAL GRASS } \\ \square \text { FORBS } & \square \text { LOCAL FORBS } \\ \square \text { WETLAND } & \square \text { LOCAL WETLAND }\end{array}$

URL: www.bigskyseeds.com

\section{Sidney}

United Agri Products

904 E. Main

Sidney, MT

59270

PHONE: (406) 482-1303

$\begin{array}{ll}\square \text { TREE } & \square \text { LOCAL TREE } \\ \square \text { SHRUB } & \square \text { LOCAL SHRUB } \\ \square \text { GRASS } & \square \text { LOCAL GRASS } \\ \square \text { FORBS } & \square \text { LOCAL FORBS } \\ \square \text { WETLAND } & \square \text { LOCAL WETLAND }\end{array}$

URL: none currently available 


\section{Stephensville}

Arbor Enterprises

1053 Park Lane

Stephensville, MT

59870

PHONE: (406) 777-0084

URL: none currently available

Earth and Wood Craftsmen

3174 Highway 93 North

Stephensville, MT

59870

PHONE: (403) 934-3670

URL: www.earthandwood.com

$\begin{array}{ll}\square \text { TREE } & \square \text { LOCAL TREE } \\ \square \text { SHRUB } & \square \text { LOCAL SHRUB } \\ \square \text { GRASS } & \square \text { LOCAL GRASS } \\ \square \text { FORBS } & \square \text { LOCAL FORBS } \\ \square \text { WETLAND } & \square \text { LOCAL WETLAND }\end{array}$

$\checkmark$ TREE

$\checkmark$ SHRUB

$\square$ GRASS

$\square$ FORBS

$\square$ WETLAND

$\checkmark$ LOCAL TREE

$\checkmark$ LOCAL SHRUB

$\square$ LOCAL GRASS

$\square$ LOCAL FORBS

$\square$ LOCAL WETLAND

\section{Stevensville}

Natures Enhancement Inc.

2980 Eastside Highway

Stevensville, MT

59870

PHONE: (406) 777-3560

$\begin{array}{ll}\nabla \text { TREE } & \square \text { LOCAL TREE } \\ \square \text { SHRUB } & \square \text { LOCAL SHRUB } \\ \square \text { GRASS } & \square \text { LOCAL GRASS } \\ \square \text { FORBS } & \square \text { LOCAL FORBS } \\ \square \text { WETLAND } & \square \text { LOCAL WETLAND }\end{array}$

URL: none currently available

\section{Sweetgrass}

West Butte Ranch

P.O. Box 32

Sweetgrass, MT

59484

PHONE: (406) 937-2281

$\begin{array}{ll}\square \text { TREE } & \square \text { LOCAL TREE } \\ \square \text { SHRUB } & \square \text { LOCAL SHRUB } \\ \square \text { GRASS } & \square \text { LOCAL GRASS } \\ \square \text { FORBS } & \square \text { LOCAL FORBS } \\ \square \text { WETLAND } & \square \text { LOCAL WETLAND }\end{array}$

URL: none currently available

\section{Thompson Falls}

Spearmint Springs

6107 Highway 200

Thompson Falls, MT

59873

PHONE: (406) 827-3074

$\begin{array}{ll}\square \text { TREE } & \square \text { LOCAL TREE } \\ \square \text { SHRUB } & \square \text { LOCAL SHRUB } \\ \square \text { GRASS } & \square \text { LOCAL GRASS } \\ \square \text { FORBS } & \square \text { LOCAL FORBS } \\ \square \text { WETLAND } & \square \text { LOCAL WETLAND }\end{array}$

URL: www.bigskytea.com

\section{Three Forks}

Circle S Seeds

Box 130

Three Forks, MT

59752

PHONE: (406) 285-3269

$\begin{array}{ll}\square \text { TREE } & \square \text { LOCAL TREE } \\ \square \text { SHRUB } & \square \text { LOCAL SHRUB } \\ \square \text { GRASS } & \square \text { LOCAL GRASS } \\ \square \text { FORBS } & \square \text { LOCAL FORBS } \\ \square \text { WETLAND } & \square \text { LOCAL WETLAND }\end{array}$

URL: none currently available 


\section{Townsend}

Bruce Seed Farm

91 Lower Deep Creek Rd.

Townsend, MT

59644

PHONE: (406) 266-3103

URL: none currently available

Townsend Seeds, Inc.

P.O. Box 1338

Townsend, MT

59644

PHONE: (406) 266-4444

$\begin{array}{ll}\square \text { TREE } & \square \text { LOCAL TREE } \\ \square \text { SHRUB } & \square \text { LOCAL SHRUB } \\ \square \text { GRASS } & \square \text { LOCAL GRASS } \\ \square \text { FORBS } & \square \text { LOCAL FORBS } \\ \square \text { WETLAND } & \square \text { LOCAL WETLAND }\end{array}$

URL: none currently available

Trout Creek

Montana Trees Inc.

Box 1505

Trout Creek, MT

59874

PHONE: (406) 827-3984

$\begin{array}{ll}\square \text { TREE } & \square \text { LOCAL TREE } \\ \square \text { SHRUB } & \square \text { LOCAL SHRUB } \\ \nabla \text { GRASS } & \square \text { LOCAL GRASS } \\ \square \text { FORBS } & \square \text { LOCAL FORBS } \\ \square \text { WETLAND } & \square \text { LOCAL WETLAND }\end{array}$

URL: none currently available

Valier

$\mathrm{CHS}$ Inc.

Box 66

Valier, MT

59486

PHONE: (406) 279-3615

$\begin{array}{ll}\square \text { TREE } & \square \text { LOCAL TREE } \\ \square \text { SHRUB } & \square \text { LOCAL SHRUB } \\ \square \text { GRASS } & \square \text { LOCAL GRASS } \\ \square \text { FORBS } & \square \text { LOCAL FORBS } \\ \square \text { WETLAND } & \square \text { LOCAL WETLAND }\end{array}$

URL: none currently available

Whitefish

Nursery for Native Plants

PO Box 1715

Whitefish, MT

59937

PHONE: (406) 752-2395

$\begin{array}{ll}\square \text { TREE } & \square \text { LOCAL TREE } \\ \square \text { SHRUB } & \square \text { LOCAL SHRUB } \\ \square \text { GRASS } & \square \text { LOCAL GRASS } \\ \square \text { FORBS } & \square \text { LOCAL FORBS } \\ \square \text { WETLAND } & \square \text { LOCAL WETLAND }\end{array}$

$\square$ WETLAND

$\square$ LOCAL WETLAND

URL: none currently available

Worden

Farmers Union Oil Co.

1545 Northern Ave.

Worden, MT

59058

PHONE: (406) 967-3100

$\begin{array}{ll}\nabla \text { TREE } & \square \text { LOCAL TREE } \\ \nabla \text { SHRUB } & \square \text { LOCAL SHRUB } \\ \nabla \text { GRASS } & \nabla \text { LOCAL GRASS } \\ \nabla \text { FORBS } & \nabla \text { LOCAL FORBS } \\ \square \text { WETLAND } & \square \text { LOCAL WETLAND }\end{array}$

$\square$ WETLAND

$\square$ LOCAL WETLAND

JRL: none currently available 


\section{North Carolina}

Angier

McLamb Nursery, Inc.

640 Greenleaf Rd

Angier, NC

27501

PHONE: (919) 894-3709

$\begin{array}{ll}\nabla \text { TREE } & \square \text { LOCAL TREE } \\ \nabla \text { SHRUB } & \square \text { LOCAL SHRUB } \\ \square \text { GRASS } & \square \text { LOCAL GRASS } \\ \square \text { FORBS } & \square \text { LOCAL FORBS } \\ \square \text { WETLAND } & \square \text { LOCAL WETLAND }\end{array}$

URL: none currently available

\section{Asheville}

Elk Mountain Nursery

P.O.Box 599

$\checkmark$ TREE

Asheville, NC

$\checkmark$ SHRUB

$\square$ LOCAL TREE

28802

$\square$ GRASS

$\square$ LOCAL SHRUB

PHONE: (828) 683-9330

$\square$ FORBS $\square$ LOCAL FORBS

$\square$ WETLAND $\square$ LOCAL WETLAND

URL: www.elk-mountain.com

Burgow

Burgow Creek Nursery \& Turf Farm, LLC

PO Box 1337

Burgow, NC

28425

PHONE: (910) 259-4788

$\begin{array}{ll}\nabla \text { TREE } & \boldsymbol{v} \text { LOCAL TREE } \\ \nabla \text { SHRUB } & \boldsymbol{v} \text { LOCAL SHRUB } \\ \square \text { GRASS } & \square \text { LOCAL GRASS } \\ \square \text { FORBS } & \square \text { LOCAL FORBS } \\ \square \text { WETLAND } & \square \text { LOCAL WETLAND }\end{array}$

URL: none currently available

\section{Chapel Hil}

Boothe Hill Wildflower Seed

921 Boothe Hill

Chapel Hill, NC

27514

PHONE: (919) 967-4091

$\begin{array}{ll}\square \text { TREE } & \square \text { LOCAL TREE } \\ \square \text { SHRUB } & \square \text { LOCAL SHRUB } \\ \square \text { GRASS } & \square \text { LOCAL GRASS } \\ \square \text { FORBS } & \square \text { LOCAL FORBS } \\ \square \text { WETLAND } & \square \text { LOCAL WETLAND }\end{array}$

URL: none currently available

Niche Gardens

1111 Dawson Rd.

Chapel Hill, NC

27516

PHONE: (919) 967-0078

$\begin{array}{ll}\nabla \text { TREE } & \square \text { LOCAL TREE } \\ \nabla \text { SHRUB } & \square \text { LOCAL SHRUB } \\ \square \text { GRASS } & \square \text { LOCAL GRASS } \\ \nabla \text { FORBS } & \square \text { LOCAL FORBS } \\ \square \text { WETLAND } & \square \text { LOCAL WETLAND }\end{array}$

URL: www.nichegdn.com

\section{Clayton}

Tarheel Native Trees

4339 Peele Rd.

$\checkmark$ TREE

Clayton, NC

SHRUB

$\checkmark$ LOCAL TREE

27520

PHONE: (919) 553-5927

$\square$ GRASS

$\square$ FORBS

v WETLAND

v LOCAL SHRUB

$\square$ LOCAL GRASS

$\square$ LOCAL FORBS

URL: none currently available 
Durham

Humphries Nursery

4712 Whitfield Rd.

Durham, NC

27707

PHONE: (919) 489-5502

$\begin{array}{ll}\square \text { TREE } & \square \text { LOCAL TREE } \\ \square \text { SHRUB } & \square \text { LOCAL SHRUB } \\ \square \text { GRASS } & \square \text { LOCAL GRASS } \\ \nabla \text { FORBS } & \square \text { LOCAL FORBS } \\ \square \text { WETLAND } & \square \text { LOCAL WETLAND }\end{array}$

URL: none currently available

Edenton

Coastal Plain Conservation Nursery

3067 Conners Dr

Edenton, NC

27932

PHONE: (252) 482-8304

$\begin{array}{ll}\nabla \text { TREE } & \nabla \text { LOCAL TREE } \\ \nabla \text { SHRUB } & \nabla \text { LOCAL SHRUB } \\ \square \text { GRASS } & \square \text { LOCAL GRASS } \\ \square \text { FORBS } & \square \text { LOCAL FORBS } \\ \nabla \text { WETLAND } & \nabla \text { LOCAL WETLAND }\end{array}$

URL: none currently available

Fayetteville

Green Biz Nursery \& Landscaping, Inc.

P.O. Box 65059

Fayetteville, NC

28306

PHONE: (800) 848-6634

$\begin{array}{ll}\nabla \text { TREE } & \square \text { LOCAL TREE } \\ \nabla \text { SHRUB } & \square \text { LOCAL SHRUB } \\ \square \text { GRASS } & \square \text { LOCAL GRASS } \\ \square \text { FORBS } & \square \text { LOCAL FORBS } \\ \square \text { WETLAND } & \square \text { LOCAL WETLAND }\end{array}$

URL: www.greenbiznursery.com

\section{Garner}

Wyatt Quarles Seed

730 US Highway $70 \mathrm{~W}$.

Garner, NC

27529

PHONE: (919) 722-4243

$\begin{array}{ll}\square \text { TREE } & \square \text { LOCAL TREE } \\ \square \text { SHRUB } & \square \text { LOCAL SHRUB } \\ \square \text { GRASS } & \square \text { LOCAL GRASS } \\ \square \text { FORBS } & \square \text { LOCAL FORBS } \\ \square \text { WETLAND } & \square \text { LOCAL WETLAND }\end{array}$

URL: wWw.wqseeds.com

\section{Goldsboro}

N.C. Division of Forest Resources

762 Claridge Nursery Road

Goldsboro. NC

27530

PHONE: (888) 628-7337

$\begin{array}{ll}\nabla \text { TREE } & \square \text { LOCAL TREE } \\ \square \text { SHRUB } & \square \text { LOCAL SHRUB } \\ \square \text { GRASS } & \square \text { LOCAL GRASS } \\ \square \text { FORBS } & \square \text { LOCAL FORBS } \\ \nabla \text { WETLAND } & \square \text { LOCAL WETLAND }\end{array}$

URL: www.dfr.state.nc.us

$\begin{array}{lll}\text { Grassy Creek } & & \\ \text { Mountain Pride Nursery } & & \\ 18777 \text { NC Hwy 194 N } & \square \text { TREE } & \square \text { LOCAL TREE } \\ \text { Grassy Creek, NC } & \square \text { SHRUB } & \square \text { LOCAL SHRUB } \\ 28631 & \square \text { GRASS } & \square \text { LOCAL GRASS } \\ \text { PHONE: (336) 384-4583 } & \square \text { FORBS } & \square \text { LOCAL FORBS } \\ & \square \text { WETLAND } & \square \text { LOCAL WETLAND }\end{array}$

URL: none currently available 
Hendersonville

Laurel Springs Nursery

401 Regal St.

Hendersonville, NC

28792

PHONE: (828) 692-4012

$\begin{array}{ll}\square \text { TREE } & \square \text { LOCAL TREE } \\ \square \text { SHRUB } & \square \text { LOCAL SHRUB } \\ \square \text { GRASS } & \square \text { LOCAL GRASS } \\ \square \text { FORBS } & \square \text { LOCAL FORBS } \\ \square \text { WETLAND } & \square \text { LOCAL WETLAND }\end{array}$

URL: none currently available

Hickory

Hawksridge Farms, Inc.

4243 Highway 127 South

Hickory, NC

28602

PHONE: (800) 874-4216

$\begin{array}{ll}\nabla \text { TREE } & \nabla \text { LOCAL TREE } \\ \nabla \text { SHRUB } & \nabla \text { LOCAL SHRUB } \\ \square \text { GRASS } & \square \text { LOCAL GRASS } \\ \square \text { FORBS } & \square \text { LOCAL FORBS } \\ \square \text { WETLAND } & \square \text { LOCAL WETLAND }\end{array}$

URL: www.hawksridgefarms.com

\begin{tabular}{|c|c|c|}
\hline \multicolumn{3}{|l|}{ Julian } \\
\hline \multicolumn{3}{|l|}{ Gilmore Plant \& Bulb Co. } \\
\hline 9154 Old 421 Road & $\checkmark$ TREE & $\checkmark$ LOCAL TREE \\
\hline Julian, NC & $\square$ SHRUB & $\square$ LOCAL SHRUB \\
\hline 27283 & $\square$ GRASS & $\square$ LOCAL GRASS \\
\hline PHONE: (336) 685-4451 & $\square$ FORBS & $\square$ LOCAL FORBS \\
\hline & $\square$ WETLAND & $\square$ LOCAL WETLAND \\
\hline
\end{tabular}

URL: www.gilmoreplantandbulb.com

Leicester

Sandy Mush Herbs

316 Surrett Cove Rd.

Leicester, NC

28748

PHONE: (828) 683-2014

$\begin{array}{ll}\nabla \text { TREE } & \square \text { LOCAL TREE } \\ \nabla \text { SHRUB } & \nabla \text { LOCAL SHRUB } \\ \nabla \text { GRASS } & \square \text { LOCAL GRASS } \\ \nabla \text { FORBS } & \nabla \text { LOCAL FORBS } \\ \square \text { WETLAND } & \square \text { LOCAL WETLAND }\end{array}$

URL: none currently available

Washington Evergreen Nursery

P.O. Box 388

Leicester, NC

28748

PHONE: (828) 683-4518

$\begin{array}{ll}\square \text { TREE } & \square \text { LOCAL TREE } \\ \square \text { SHRUB } & \square \text { LOCAL SHRUB } \\ \square \text { GRASS } & \square \text { LOCAL GRASS } \\ \square \text { FORBS } & \square \text { LOCAL FORBS } \\ \square \text { WETLAND } & \square \text { LOCAL WETLAND }\end{array}$

URL: none currently available

\section{Marion}

WE-DU Nurseries

Rt.5, Box 724"

Marion, NC

28752

PHONE: (828) 738-8300

$\begin{array}{ll}\nabla \text { TREE } & \square \text { LOCAL TREE } \\ \nabla \text { SHRUB } & \square \text { LOCAL SHRUB } \\ \square \text { GRASS } & \square \text { LOCAL GRASS } \\ \nabla \text { FORBS } & \nabla \text { LOCAL FORBS } \\ \nabla \text { WETLAND } & \square \text { LOCAL WETLAND }\end{array}$

URL: www.we-du.com 


\section{Marshall}

Griffeys Nursery

4920 NE US Hwy. $25 \& 70$

Marshall, NC

28753

PHONE: (828) 656-2723

$\begin{array}{ll}\nabla \text { TREE } & \nabla \text { LOCAL TREE } \\ \nabla \text { SHRUB } & \nabla \text { LOCAL SHRUB } \\ \square \text { GRASS } & \square \text { LOCAL GRASS } \\ \nabla \text { FORBS } & \square \text { LOCAL FORBS } \\ \square \text { WETLAND } & \square \text { LOCAL WETLAND }\end{array}$

URL: none currently available

Wildflower Nursery

1680 Hwy. 25-70

Marshall, NC

28753

PHONE: (828) 656-2723

$\begin{array}{ll}\nabla \text { TREE } & \square \text { LOCAL TREE } \\ \nabla \text { SHRUB } & \square \text { LOCAL SHRUB } \\ \square \text { GRASS } & \square \text { LOCAL GRASS } \\ \nabla \text { FORBS } & \square \text { LOCAL FORBS } \\ \square \text { WETLAND } & \square \text { LOCAL WETLAND }\end{array}$

URL: none currently available

\section{Murphy}

Hanging Dog Valley Nursery

2600 Boiling Spring Road

Murphy, NC

28906

PHONE: (704) 837-7921

$\begin{array}{ll}\nabla \text { TREE } & \square \text { LOCAL TREE } \\ \nabla \text { SHRUB } & \boldsymbol{\nabla} \text { LOCAL SHRUB } \\ \square \text { GRASS } & \square \text { LOCAL GRASS } \\ \square \text { FORBS } & \square \text { LOCAL FORBS } \\ \square \text { WETLAND } & \square \text { LOCAL WETLAND }\end{array}$

URL: none currently available

\section{Pineola}

Gardens of the Blue Ridge

PO Box 10

Pineola, NC

28662

PHONE: (704) 733-2417

$\begin{array}{ll}\nabla \text { TREE } & \square \text { LOCAL TREE } \\ \nabla \text { SHRUB } & \nabla \text { LOCAL SHRUB } \\ \square \text { GRASS } & \square \text { LOCAL GRASS } \\ \square \text { FORBS } & \square \text { LOCAL FORBS } \\ \square \text { WETLAND } & \square \text { LOCAL WETLAND }\end{array}$

URL: www.gardensotheblueridge.com

\section{Pittsboro}

Cure Nursery

880 Buteo Rd

Pittsboro, NC

27312

PHONE: (919) 542-6186

$\begin{array}{ll}\nabla \text { TREE } & \square \text { LOCAL TREE } \\ \nabla \text { SHRUB } & \square \text { LOCAL SHRUB } \\ \square \text { GRASS } & \square \text { LOCAL GRASS } \\ \square \text { FORBS } & \square \text { LOCAL FORBS } \\ \square \text { WETLAND } & \square \text { LOCAL WETLAND }\end{array}$

URL: none currently available

\section{Mellow Marsh Farm}

205 Anolis Rd

Pittsboro, NC

27312

PHONE: (919) 542-3532

$\begin{array}{ll}\nabla \text { TREE } & \checkmark \text { LOCAL TREE } \\ \nabla \text { SHRUB } & \nabla \text { LOCAL SHRUB } \\ \nabla \text { GRASS } & \nabla \text { LOCAL GRASS } \\ \nabla \text { FORBS } & \checkmark \text { LOCAL FORBS } \\ \nabla \text { WETLAND } & \checkmark \text { LOCAL WETLAND }\end{array}$

URL: www.mellowmarshfarm.com 
Take Root Ferns

220 Blakes Dr.

Pittsboro, NC

27312

PHONE: (919) 967-9515

$\begin{array}{ll}\square \text { TREE } & \square \text { LOCAL TREE } \\ \square \text { SHRUB } & \square \text { LOCAL SHRUB } \\ \square \text { GRASS } & \square \text { LOCAL GRASS } \\ \square \text { FORBS } & \square \text { LOCAL FORBS } \\ \square \text { WETLAND } & \square \text { LOCAL WETLAND }\end{array}$

URL: none currently available

Raleigh

Plant Delights Nursery

9241 Sauls Rd.

Raleigh, NC

27603

PHONE: (919) 772-4794

$\begin{array}{ll}\nabla \text { TREE } & \square \text { LOCAL TREE } \\ \square \text { SHRUB } & \square \text { LOCAL SHRUB } \\ \nabla \text { GRASS } & \nabla \text { LOCAL GRASS } \\ \nabla \text { FORBS } & \nabla \text { LOCAL FORBS } \\ \square \text { WETLAND } & \square \text { LOCAL WETLAND }\end{array}$

URL: www.plantdel.com

Rougemont

Hoffman Nursery

5520 Bahama Rd.

Rougemont, NC

27572

PHONE: (919) 479-6620

$\begin{array}{ll}\square \text { TREE } & \square \text { LOCAL TREE } \\ \square \text { SHRUB } & \square \text { LOCAL SHRUB } \\ \nabla \text { GRASS } & \square \text { LOCAL GRASS } \\ \square \text { FORBS } & \square \text { LOCAL FORBS } \\ \nabla \text { WETLAND } & \square \text { LOCAL WETLAND }\end{array}$

URL: www.hoffmannursery.com

Sylva

Greens Creek Nursery

Rt. 2, Box 113A

Sylva, NC

28779

PHONE: (828) 586-2726

$\begin{array}{ll}\nabla \text { TREE } & \square \text { LOCAL TREE } \\ \nabla \text { SHRUB } & \nabla \text { LOCAL SHRUB } \\ \square \text { GRASS } & \square \text { LOCAL GRASS } \\ \square \text { FORBS } & \square \text { LOCAL FORBS } \\ \square \text { WETLAND } & \square \text { LOCAL WETLAND }\end{array}$

URL: none currently available

Warrensville

Lamtree Farm

2323 Copeland Rd.

Warrensville, NC

28693

PHONE: (800) 537-5268

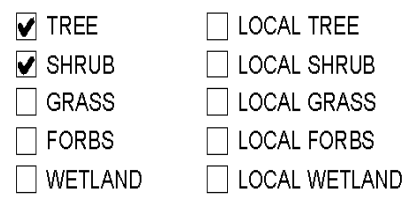

URL: none currently available

Washington

Weyerhauser $\mathrm{Co}$.

1123 Dinah's Landing Rd

Washington, NC

27889

PHONE: (800) 344-0399

$\begin{array}{ll}\square \text { TREE } & \square \text { LOCAL TREE } \\ \square \text { SHRUB } & \square \text { LOCAL SHRUB } \\ \square \text { GRASS } & \square \text { LOCAL GRASS } \\ \square \text { FORBS } & \square \text { LOCAL FORBS } \\ \square \text { WETLAND } & \square \text { LOCAL WETLAND }\end{array}$

URL: www.weverhauser.com 


\section{West End}

Carolina Greenery

375 Carthage Rd.

West End, NC

27376

PHONE: (910) 947-3150

$\begin{array}{ll}\nabla \text { TREE } & \checkmark \text { LOCAL TREE } \\ \nabla \text { SHRUB } & \checkmark \text { LOCAL SHRUB } \\ \nabla \text { GRASS } & \checkmark \text { LOCAL GRASS } \\ \nabla \text { FORBS } & \square \text { LOCAL FORBS } \\ \nabla \text { WETLAND } & \square \text { LOCAL WETLAND }\end{array}$

URL: www.carolinagreenery.com

\section{Winston Salem}

Lofts Seeds, Inc.

P.O. Box 26223

Winston Salem, NC

27114

$\begin{array}{ll}\square \text { TREE } & \square \text { LOCAL TREE } \\ \square \text { SHRUB } & \square \text { LOCAL SHRUB } \\ \square \text { GRASS } & \square \text { LOCAL GRASS } \\ \square \text { FORBS } & \square \text { LOCAL FORBS } \\ \square \text { WETLAND } & \square \text { LOCAL WETLAND }\end{array}$

PHONE: (800) 526-3890

$\square$ WETLAND $\square$ LOCAL WETLAND

URL: wnw.turf.com

Yadkinville

Fern Valley Farms, Inc.

1624 Fern Valley Rd.

Yadkinville, NC

27055

PHONE: (336) 463-2412

$\begin{array}{ll}\square \text { TREE } & \square \text { LOCAL TREE } \\ \square \text { SHRUB } & \square \text { LOCAL SHRUB } \\ \square \text { GRASS } & \square \text { LOCAL GRASS } \\ \square \text { FORBS } & \square \text { LOCAL FORBS } \\ \square \text { WETLAND } & \square \text { LOCAL WETLAND }\end{array}$

URL: none currently available

\section{North Dakota}

Bismarck

Chesak Seed House

220 N. 23 rd St.

Bismarck, ND

58501

PHONE: (701) 223-0391

$\begin{array}{ll}\square \text { TREE } & \square \text { LOCAL TREE } \\ \square \text { SHRUB } & \square \text { LOCAL SHRUB } \\ \nabla \text { GRASS } & \square \text { LOCAL GRASS } \\ \nabla \text { FORBS } & \square \text { LOCAL FORBS } \\ \nabla \text { WETLAND } & \square \text { LOCAL WETLAND }\end{array}$

URL: none currently available

Lincoln-Oakes Nursery

Box 1601

Bismarck, ND

58502

PHONE: (701) 223-8575

$\begin{array}{ll}\nabla \text { TREE } & \square \text { LOCAL TREE } \\ \nabla \text { SHRUB } & \square \text { LOCAL SHRUB } \\ \square \text { GRASS } & \square \text { LOCAL GRASS } \\ \square \text { FORBS } & \square \text { LOCAL FORBS } \\ \nabla \text { WETLAND } & \square \text { LOCAL WETLAND }\end{array}$

URL: www.lincolnoakes.com

Plant Materials Center

3308 University Dr.

Bismarck, ND

58504

PHONE: (701) 250-4330

$\begin{array}{ll}\square \text { TREE } & \square \text { LOCAL TREE } \\ \square \text { SHRUB } & \square \text { LOCAL SHRUB } \\ \square \text { GRASS } & \square \text { LOCAL GRASS } \\ \square \text { FORBS } & \square \text { LOCAL FORBS } \\ \square \text { WETLAND } & \square \text { LOCAL WETLAND }\end{array}$

URL: http://plant-materials.nrcs.usda.gov/ndpmc/ 
S \& B Landscaping \& Nursery

$\begin{array}{lll}\text { 1400 Airport Rd. } & \square \text { TREE } & \square \text { LOCAL TREE } \\ \text { Bismarck, ND } & \square \text { SHRUB } & \square \text { LOCAL SHRUB } \\ 58504 & \square \text { GRASS } & \square \text { LOCAL GRASS } \\ \text { PHONE: (701) 224-0100 } & \square \text { FORBS } & \square \text { LOCAL FORBS } \\ & \square \text { WETLAND } & \square \text { LOCAL WETLAND }\end{array}$

URL: none currently available

Bottineau

North Dakota Forest Service

307 First Street East

Bottineau, ND

58318

PHONE: (701) 228-5422

$\begin{array}{ll}\nabla \text { TREE } & \square \text { LOCAL TREE } \\ \square \text { SHRUB } & \square \text { LOCAL SHRUB } \\ \square \text { GRASS } & \square \text { LOCAL GRASS } \\ \square \text { FORBS } & \square \text { LOCAL FORBS } \\ \square \text { WETLAND } & \square \text { LOCAL WETLAND }\end{array}$

URL: http://www.ndsu.nodak.edu/ndsu/bakkenforest/

Carrington

Reimers Seed Co.

7074 Highway 9

Carrington, ND

58421

PHONE: (701) 652-3322

$\begin{array}{ll}\square \text { TREE } & \square \text { LOCAL TREE } \\ \square \text { SHRUB } & \square \text { LOCAL SHRUB } \\ \square \text { GRASS } & \square \text { LOCAL GRASS } \\ \square \text { FORBS } & \square \text { LOCAL FORBS } \\ \square \text { WETLAND } & \square \text { LOCAL WETLAND }\end{array}$

URL: none currently available

\section{Casselton}

Unity Seed $C_{0}$.

P.O. Box 567

Casselton, ND

58012

PHONE: (701) 347-5355

$\begin{array}{ll}\square \text { TREE } & \square \text { LOCAL TREE } \\ \square \text { SHRUB } & \square \text { LOCAL SHRUB } \\ \square \text { GRASS } & \square \text { LOCAL GRASS } \\ \square \text { FORBS } & \square \text { LOCAL FORBS } \\ \square \text { WETLAND } & \square \text { LOCAL WETLAND }\end{array}$

URL: www.unityseed.com

Cogswell

Heimbuch Farms

Rt.1, Box 70"

Cogswell, ND

58017

PHONE: (701) 724-3293

$\begin{array}{ll}\square \text { TREE } & \square \text { LOCAL TREE } \\ \square \text { SHRUB } & \square \text { LOCAL SHRUB } \\ \nabla \text { GRASS } & \square \text { LOCAL GRASS } \\ \square \text { FORBS } & \square \text { LOCAL FORBS } \\ \square \text { WETLAND } & \square \text { LOCAL WETLAND }\end{array}$

URL: none currently available

\section{Coleharbor}

Robinson Stock Farm

Rt. 1, Box 5C"

Coleharbor, ND

58531

PHONE: (701) 442-5418

$\begin{array}{ll}\square \text { TREE } & \square \text { LOCAL TREE } \\ \square \text { SHRUB } & \square \text { LOCAL SHRUB } \\ \square \text { GRASS } & \square \text { LOCAL GRASS } \\ \square \text { FORBS } & \square \text { LOCAL FORBS } \\ \square \text { WETLAND } & \square \text { LOCAL WETLAND }\end{array}$

URL: none currently available 
Dickinson

Hammel Ag and Seed Supply Inc.

664 East Broadway Street

Dickinson, ND

58601

PHONE: (701) 483-3565

$\begin{array}{ll}\square \text { TREE } & \square \text { LOCAL TREE } \\ \square \text { SHRUB } & \square \text { LOCAL SHRUB } \\ \square \text { GRASS } & \square \text { LOCAL GRASS } \\ \square \text { FORBS } & \square \text { LOCAL FORBS } \\ \square \text { WETLAND } & \square \text { LOCAL WETLAND }\end{array}$

URL: none currently available

Hettinger

Knudson Feeds Inc

304 2nd Ave. S.

Hettinger, ND

58639

$\square$ SHRUB $\quad \square$ LOCAL SHRUB

PHONE: (701) 567-2444

$\square$ GRASS $\square$ LOCAL GRASS

$\begin{array}{ll}\square \text { FORBS } & \square \text { LOCAL FORBS } \\ \square \text { WETLAND } & \square \text { LOCAL WETLAND }\end{array}$

URL: http://Www .kfi4u.com/

\begin{tabular}{|c|c|c|}
\hline & & \\
\hline \multicolumn{3}{|l|}{ Land O Lakes Seed Plant } \\
\hline Box 1291 & $\square$ TREE & $\square$ LOCAL TREE \\
\hline Minot, ND & $\square$ SHRUB & $\square$ LOCAL SHRUB \\
\hline 58701 & $\nabla$ GRASS & $\square$ LOCAL GRASS \\
\hline \multirow[t]{2}{*}{ PHONE: (701) 852-3556 } & $\square$ FORBS & $\square$ LOCAL FORBS \\
\hline & $\square$ WETLAND & $\square$ LOCAL WETLAND \\
\hline
\end{tabular}

URL: www.croplangenetics.com

Reeder

Southwest Grain

P.O. Box 157

Reeder, ND

58649

PHONE: (701) 853-2302

$\begin{array}{ll}\square \text { TREE } & \square \text { LOCAL TREE } \\ \square \text { SHRUB } & \square \text { LOCAL SHRUB } \\ \square \text { GRASS } & \square \text { LOCAL GRASS } \\ \square \text { FORBS } & \square \text { LOCAL FORBS } \\ \square \text { WETLAND } & \square \text { LOCAL WETLAND }\end{array}$

URL: wWw.Swgrain.com

West Fargo

Agassiz Seed

4457 th St. NW

West Fargo, ND

58078

PHONE: (701) 282-8118

$\begin{array}{ll}\square \text { TREE } & \square \text { LOCAL TREE } \\ \square \text { SHRUB } & \square \text { LOCAL SHRUB } \\ \square \text { GRASS } & \square \text { LOCAL GRASS } \\ \square \text { FORBS } & \square \text { LOCAL FORBS } \\ \square \text { WETLAND } & \square \text { LOCAL WETLAND }\end{array}$

URL: www.agassizseed.com

\section{Williston}

Land OLakes Seed Plant

Box 155

Williston, ND

58801

$\square$ TREE

$\square$ SHRUB

$\checkmark$ GRASS

$\square$ LOCAL TREE

$\square$ FORBS

$\square$ LOCAL SHRUB

PHONE: (701) 572-5180

$\square$ WETLAND $\square$ LOCAL WETLAND

URL: www.croplangenetics.com 


\section{Nebraska}

Bridgeport

Laux Seed Farm

HC 85, Box 48"

Bridgeport, NE

69336

$\begin{array}{ll}\square \text { TREE } & \square \text { LOCAL TREE } \\ \square \text { SHRUB } & \square \text { LOCAL SHRUB } \\ \square \text { GRASS } & \square \text { LOCAL GRASS } \\ \square \text { FORBS } & \square \text { LOCAL FORBS } \\ \square \text { WETLAND } & \square \text { LOCAL WETLAND }\end{array}$

PHONE: (308) 262-0512

$\square$ WETLAND $\square$ LOCAL WETLAND

URL: none currently available

\section{Bristow}

P.E. Allen Farm Supply

$\begin{array}{lll}\text { Rt.2, Box } 8 & \square \text { TREE } & \square \text { LOCAL TREE } \\ \text { Bristow, NE } & \nabla \text { SHRUB } & \nabla \text { LOCAL SHRUB } \\ 68719 & \square \text { GRASS } & \square \text { LOCAL GRASS } \\ \text { PHONE: (402) 583-9924 } & \square \text { FORBS } & \square \text { LOCAL FORBS } \\ & \square \text { WETLAND } & \square \text { LOCAL WETLAND }\end{array}$

URL: none currently available

\section{Broken Bow}

Arrow Seed $\mathrm{C}_{0}$, Inc.

Box 722

Broken Bow, NE

68822

PHONE: (800) 622-4727

$\begin{array}{ll}\square \text { TREE } & \square \text { LOCAL TREE } \\ \square \text { SHRUB } & \square \text { LOCAL SHRUB } \\ \nabla \text { GRASS } & \nabla \text { LOCAL GRASS } \\ \square \text { FORBS } & \square \text { LOCAL FORBS } \\ \square \text { WETLAND } & \square \text { LOCAL WETLAND }\end{array}$

URL: www.arrowseed.com

\section{Clarkson}

Bluebird Nursery, Inc.

515 Bryan P.O. Box 460

Clarkson, NE

68629

PHONE: (402) 892-3457

$\begin{array}{ll}\square \text { TREE } & \square \text { LOCAL TREE } \\ \square \text { SHRUB } & \square \text { LOCAL SHRUB } \\ \nabla \text { GRASS } & \square \text { LOCAL GRASS } \\ \nabla \text { FORBS } & \square \text { LOCAL FORBS } \\ \nabla \text { WETLAND } & \nabla \text { LOCAL WETLAND }\end{array}$

URL: www.bluebirdnursery.com

\section{Crawford}

Heritage Seed

Box 544

Crawford, NE

69339

PHONE: (308) 665-1672

$\begin{array}{ll}\square \text { TREE } & \square \text { LOCAL TREE } \\ \square \text { SHRUB } & \square \text { LOCAL SHRUB } \\ \square \text { GRASS } & \square \text { LOCAL GRASS } \\ \square \text { FORBS } & \square \text { LOCAL FORBS } \\ \square \text { WETLAND } & \square \text { LOCAL WETLAND }\end{array}$

URL: none currently available

\section{Fairbury}

Knobel Seeds

72055 567th Ave.

Fairbury, NE

68352

PHONE: (402) 446-7394

$\begin{array}{ll}\square \text { TREE } & \square \text { LOCAL TREE } \\ \square \text { SHRUB } & \square \text { LOCAL SHRUB } \\ \nabla \text { GRASS } & \square \text { LOCAL GRASS } \\ \square \text { FORBS } & \square \text { LOCAL FORBS } \\ \square \text { WETLAND } & \square \text { LOCAL WETLAND }\end{array}$

URL: none currently available 
Falls City

Gamagrass Seed Co.

Rt.1, Box 111A"

Falls City, NE

68355

$\square$ TREE $\square$ LOCAL TREE

PHONE: (800) 367-2879

$\square$ SHRUB $\square$ LOCAL SHRUB

$\nabla$ GRASS $\quad \nabla$ LOCAL GRASS

$\square$ FORBS $\square$ LOCAL FORBS

$\nabla$ WETLAND $\nabla$ LOCAL WETLAND

URL: www.gamagrass.com

Franklin

Cline Seed \& Farm Supply

PO Box 192

Franklin, NE

68939

PHONE: (308) 425-3617

$\begin{array}{ll}\square \text { TREE } & \square \text { LOCAL TREE } \\ \square \text { SHRUB } & \square \text { LOCAL SHRUB } \\ \square \text { GRASS } & \square \text { LOCAL GRASS } \\ \square \text { FORBS } & \square \text { LOCAL FORBS } \\ \square \text { WETLAND } & \square \text { LOCAL WETLAND }\end{array}$

URL: none currently available

Haigler

Olson Livestock \& Seed

HC 67 Box 12

Haigler, NE

69030

PHONE: (308) 297-3283

$\begin{array}{ll}\square \text { TREE } & \square \text { LOCAL TREE } \\ \square \text { SHRUB } & \square \text { LOCAL SHRUB } \\ \square \text { GRASS } & \square \text { LOCAL GRASS } \\ \square \text { FORBS } & \square \text { LOCAL FORBS } \\ \square \text { WETLAND } & \square \text { LOCAL WETLAND }\end{array}$

URL: none currently available

Halsey

Charles E. Bessey Nursery

PO Box 39

$\boldsymbol{\nabla}$ TREE $\quad \boldsymbol{v}$ LOCAL TREE

Halsey, NE

$\checkmark$ SHRUB

$\checkmark$ LOCAL SHRUB

69142-003

$\square$ GRASS

$\square$ LOCAL GRASS

PHONE: (308) 533-2257

$\begin{array}{ll}\square \text { FORBS } & \square \text { LOCAL FORBS } \\ \nabla \text { WETLAND } & \nabla \text { LOCAL WETLAND }\end{array}$

URL: http://Www ff.fed.us/2/nebraska/units/bn/nursery.html

Lincoln

Miller Seed \& Supply

1600 Cornhusker Highway P.O. Box 81823

Lincoln, NE

68501

$\begin{array}{ll}\square \text { TREE } & \square \text { LOCAL TREE } \\ \square \text { SHRUB } & \square \text { LOCAL SHRUB } \\ \nabla \text { GRASS } & \square \text { LOCAL GRASS } \\ \nabla \text { FORBS } & \square \text { LOCAL FORBS } \\ \square \text { WETLAND } & \square \text { LOCAL WETLAND }\end{array}$

PHONE: (402) 438-1068

$\square$ WETLAND $\square$ LOCAL WETLAND

URL: none currently available

\section{Murdock}

Herman Platt

24313 Mill Rd

Murdock, NE

68407

$\begin{array}{ll}\square \text { TREE } & \square \text { LOCAL TREE } \\ \square \text { SHRUB } & \square \text { LOCAL SHRUB } \\ \square \text { GRASS } & \square \text { LOCAL GRASS } \\ \square \text { FORBS } & \square \text { LOCAL FORBS } \\ \square \text { WETLAND } & \square \text { LOCAL WETLAND }\end{array}$

PHONE: (402) 867-2428

$\square$ WETLAND $\square$ LOCAL WETLAND

URL: none currently available 
Stock Seed Farms

28008 Mill Rd

Murdock, NE

68407

PHONE: (402) 867-3771

$\begin{array}{ll}\square \text { TREE } & \square \text { LOCAL TREE } \\ \square \text { SHRUB } & \square \text { LOCAL SHRUB } \\ \nabla \text { GRASS } & \square \text { LOCAL GRASS } \\ \square \text { FORBS } & \square \text { LOCAL FORBS } \\ \square \text { WETLAND } & \square \text { LOCAL WETLAND }\end{array}$

URL: www.stockseed.com

Omaha

DeGiorgi Seed Co.

6011 N St.

Omaha, NE

68117

PHONE: (402) 731-3901

$\begin{array}{ll}\square \text { TREE } & \square \text { LOCAL TREE } \\ \square \text { SHRUB } & \square \text { LOCAL SHRUB } \\ \nabla \text { GRASS } & \square \text { LOCAL GRASS } \\ \nabla \text { FORBS } & \square \text { LOCAL FORBS } \\ \square \text { WETLAND } & \square \text { LOCAL WETLAND }\end{array}$

URL: http://www.degiorgiseed.com/

United Seeds, Inc.

75th \& Burlington

Omaha, NE

68127

$\begin{array}{ll}\square \text { TREE } & \square \text { LOCAL TREE } \\ \square \text { SHRUB } & \square \text { LOCAL SHRUB } \\ \nabla \text { GRASS } & \square \text { LOCAL GRASS } \\ \nabla \text { FORBS } & \square \text { LOCAL FORBS } \\ \nabla \text { WETLAND } & \square \text { LOCAL WETLAND }\end{array}$

PHONE: (402) 331-4800

$\checkmark$ WETLAND $\square$ LOCAL WETLAND

URL: none currently available

Wayne

Garden Perennials

Rt 1, Box 164

Wayne, NE

68787

PHONE: (402) 375-3615

$\begin{array}{ll}\square \text { TREE } & \square \text { LOCAL TREE } \\ \square \text { SHRUB } & \square \text { LOCAL SHRUB } \\ \square \text { GRASS } & \square \text { LOCAL GRASS } \\ \square \text { FORBS } & \square \text { LOCAL FORBS } \\ \square \text { WETLAND } & \square \text { LOCAL WETLAND }\end{array}$

URL: none currently available

\begin{tabular}{|c|c|c|}
\hline \multicolumn{3}{|l|}{ Wymore } \\
\hline \multicolumn{3}{|l|}{ Husa Seed Farms } \\
\hline RR 2 & $\square$ TREE & $\square$ LOCAL TREE \\
\hline Wymore, NE & $\square$ SHRUB & $\square$ LOCAL SHRUB \\
\hline 68466 & $\checkmark$ GRASS & $\square$ LOCAL GRASS \\
\hline PHONE: (402) 674-3188 & $\square$ FORBS & $\square$ LOCAL FORBS \\
\hline & $\square$ WETLAND & $\square$ LOCAL WETLAND \\
\hline
\end{tabular}

URL: none currently available

\section{New Hampshire}

Chichester

Millican Nurseries, Inc.

187 Pleasant St.

Chichester, NH

03234

PHONE: (603) 435-6660

$\begin{array}{ll}\nabla \text { TREE } & \square \text { LOCAL TREE } \\ \nabla \text { SHRUB } & \square \text { LOCAL SHRUB } \\ \square \text { GRASS } & \square \text { LOCAL GRASS } \\ \square \text { FORBS } & \square \text { LOCAL FORBS } \\ \square \text { WETLAND } & \square \text { LOCAL WETLAND }\end{array}$

URL: www.millicannurseriesinc.com 


\section{Concord}

L.A. Brochu \& Son Nurseries, Inc.

121 Commercial St.

Concord, NH

03301

PHONE: (603) 224-4350

$\begin{array}{ll}\nabla \text { TREE } & \square \text { LOCAL TREE } \\ \nabla \text { SHRUB } & \square \text { LOCAL SHRUB } \\ \nabla \text { GRASS } & \square \text { LOCAL GRASS } \\ \square \text { FORBS } & \square \text { LOCAL FORBS } \\ \square \text { WETLAND } & \square \text { LOCAL WETLAND }\end{array}$

URL: none currently available

\section{NH State Forest Nursery}

PO Box 1856

Concord, NH

03302-185

PHONE: (603) 271-3456

$\begin{array}{ll}\nabla \text { TREE } & \square \text { LOCAL TREE } \\ \square \text { SHRUB } & \square \text { LOCAL SHRUB } \\ \square \text { GRASS } & \square \text { LOCAL GRASS } \\ \square \text { FORBS } & \square \text { LOCAL FORBS } \\ \square \text { WETLAND } & \square \text { LOCAL WETLAND }\end{array}$

URL: http:/Nwww. nhnursery.com/

\section{East Kingston}

Oakridge Nurseries

P.O.Box 182

East Kingston, $\mathrm{NH}$

03872

PHONE: (603) 642-7339

$\begin{array}{ll}\square \text { TREE } & \square \text { LOCAL TREE } \\ \square \text { SHRUB } & \square \text { LOCAL SHRUB } \\ \square \text { GRASS } & \square \text { LOCAL GRASS } \\ \square \text { FORBS } & \square \text { LOCAL FORBS } \\ \square \text { WETLAND } & \square \text { LOCAL WETLAND }\end{array}$

URL: none currently available

\section{Hampton Falls}

Dodges Agway

116 Lafayette Rd.

Hampton Falls, NH

03844

PHONE: (603) 926-2253

$\begin{array}{ll}\square \text { TREE } & \square \text { LOCAL TREE } \\ \square \text { SHRUB } & \square \text { LOCAL SHRUB } \\ \square \text { GRASS } & \square \text { LOCAL GRASS } \\ \square \text { FORBS } & \square \text { LOCAL FORBS } \\ \square \text { WETLAND } & \square \text { LOCAL WETLAND }\end{array}$

URL: none currently available

Kingston

Kingston Nursery

P.O. Box 696

Kingston, $\mathrm{NH}$

03848

PHONE: (603) 642-5216

$\begin{array}{ll}\square \text { TREE } & \square \text { LOCAL TREE } \\ \square \text { SHRUB } & \square \text { LOCAL SHRUB } \\ \square \text { GRASS } & \square \text { LOCAL GRASS } \\ \square \text { FORBS } & \square \text { LOCAL FORBS } \\ \square \text { WETLAND } & \square \text { LOCAL WETLAND }\end{array}$

URL: none currently available

\section{Monroe}

Yankee Gardener Greenhouses and Nursery

132 Smutty Hollow Rd

Monroe, $\mathrm{NH}$

03771

PHONE: (603) 638-2809

$\begin{array}{ll}\square \text { TREE } & \square \text { LOCAL TREE } \\ \square \text { SHRUB } & \square \text { LOCAL SHRUB } \\ \square \text { GRASS } & \square \text { LOCAL GRASS } \\ \square \text { FORBS } & \square \text { LOCAL FORBS } \\ \square \text { WETLAND } & \square \text { LOCAL WETLAND }\end{array}$

URL: none currently available 
Newbury

Lyons Family Nursery

161 Morse Hill Ro

Newbury, NH

03255

PHONE: (603) 938-5398

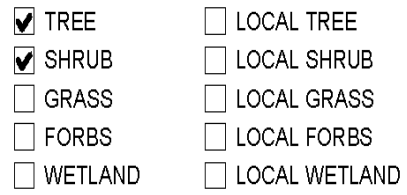

URL: none currently available

\section{Portsmouth}

Rolling Green Nursery

PO Box 4093

$\begin{array}{ll}\square \text { TREE } & \square \text { LOCAL TREE } \\ \nabla \text { SHRUB } & \nabla \text { LOCAL SHRUB } \\ \square \text { GRASS } & \square \text { LOCAL GRASS } \\ \nabla \text { FORBS } & \square \text { LOCAL FORBS } \\ \square \text { WETLAND } & \square \text { LOCAL WETLAND }\end{array}$

Portsmouth, $\mathrm{NH}$

03802

PHONE: (603) 436-2732

$\square$ WETLAND $\square$ LOCAL WETLAND

URL: none currently available

Rochester

ELFS Landscaping

PO Box 7226

Rochester, NH

03839

PHONE: (603) 332-8324

$\begin{array}{ll}\nabla \text { TREE } & \square \text { LOCAL TREE } \\ \nabla \text { SHRUB } & \nabla \text { LOCAL SHRUB } \\ \nabla \text { GRASS } & \nabla \text { LOCAL GRASS } \\ \nabla \text { FORBS } & \square \text { LOCAL FORBS } \\ \nabla \text { WETLAND } & \square \text { LOCAL WETLAND }\end{array}$

URL: none currently available

\section{New Jersey}

Allentown

Farmers Brokerage and Supply

P.O. Box 458

Allentown, NJ

08501

PHONE: (609) 259-7323

$\begin{array}{ll}\square \text { TREE } & \square \text { LOCAL TREE } \\ \square \text { SHRUB } & \square \text { LOCAL SHRUB } \\ \square \text { GRASS } & \square \text { LOCAL GRASS } \\ \square \text { FORBS } & \square \text { LOCAL FORBS } \\ \square \text { WETLAND } & \square \text { LOCAL WETLAND }\end{array}$

URL: www.fbands.com

Princeton Nurseries

P.O.Box 185

Allentown, $\mathrm{NJ}$

08501

PHONE: (609) 259-7671

$$
\begin{array}{ll}
\nabla \text { TREE } & \nabla \text { LOCAL TREE } \\
\nabla \text { SHRUB } & \square \text { LOCAL SHRUB } \\
\nabla \text { GRASS } & \square \text { LOCAL GRASS } \\
\nabla \text { FORBS } & \square \text { LOCAL FORBS } \\
\square \text { WETLAND } & \square \text { LOCAL WETLAN }
\end{array}
$$

URL: www.princetonnurseries.com

\section{Bound Brook}

Lofts Seeds, Inc.

Box 146

Bound Brook, NJ

08805

PHONE: (800) 526-3890

$\begin{array}{ll}\square \text { TREE } & \square \text { LOCAL TREE } \\ \square \text { SHRUB } & \square \text { LOCAL SHRUB } \\ \square \text { GRASS } & \square \text { LOCAL GRASS } \\ \square \text { FORBS } & \square \text { LOCAL FORBS } \\ \square \text { WETLAND } & \square \text { LOCAL WETLAND }\end{array}$

URL: www.turf.com 


\section{Cape May}

Churchs Greenhouse \& Nursery

522 Seashore Rd.

Cape May, NJ

08204

PHONE: (609) 884-3927

URL: www.churchsbeachgrass.com

\section{Plant Materials Center}

1536 Rt. $9 \mathrm{~N}$

Cape May, NJ

08210

PHONE: (609) 465-5901

URL: http://plant-materials.nrcs.usda.gov/nipmc/

$\begin{array}{ll}\nabla \text { TREE } & \square \text { LOCAL TREE } \\ \nabla \text { SHRUB } & \square \text { LOCAL SHRUB } \\ \square \text { GRASS } & \square \text { LOCAL GRASS } \\ \nabla \text { FORBS } & \square \text { LOCAL FORBS } \\ \square \text { WETLAND } & \square \text { LOCAL WETLAND }\end{array}$

$\checkmark$ WETLAND

$\begin{array}{ll}\square \text { TREE } & \square \text { LOCAL TREE } \\ \square \text { SHRUB } & \square \text { LOCAL SHRUB } \\ \square \text { GRASS } & \square \text { LOCAL GRASS } \\ \square \text { FORBS } & \square \text { LOCAL FORBS } \\ \square \text { WETLAND } & \square \text { LOCAL WETLAND }\end{array}$

Clifton

Terre Company

206 Delawanna Ave.

Cliton, NJ

07014

PHONE: (201) 473-3393

$\begin{array}{ll}\nabla \text { TREE } & \square \text { LOCAL TREE } \\ \nabla \text { SHRUB } & \square \text { LOCAL SHRUB } \\ \nabla \text { GRASS } & \square \text { LOCAL GRASS } \\ \nabla \text { FORBS } & \square \text { LOCAL FORBS } \\ \nabla \text { WETLAND } & \square \text { LOCAL WETLAND }\end{array}$

URL: www.terrecompany.com

\section{Columbus}

Croshaw Nursery

Box 339, Mill Lane

Columbus, NJ

08022

PHONE: (609) 298-0477

$\begin{array}{ll}\nabla \text { TREE } & \square \text { LOCAL TREE } \\ \nabla \text { SHRUB } & \nabla \text { LOCAL SHRUB } \\ \square \text { GRASS } & \square \text { LOCAL GRASS } \\ \square \text { FORBS } & \square \text { LOCAL FORBS } \\ \square \text { WETLAND } & \square \text { LOCAL WETLAND }\end{array}$

URL: www.croshawnursery.com

Pinelands Nursery \& Supply

323 Island Rd.

Columbus, NJ

08022

PHONE: (609) 291-9486

$\begin{array}{ll}\nabla \text { TREE } & \square \text { LOCAL TREE } \\ \nabla \text { SHRUB } & \square \text { LOCAL SHRUB } \\ \nabla \text { GRASS } & \square \text { LOCAL GRASS } \\ \nabla \text { FORBS } & \square \text { LOCAL FORBS } \\ \nabla \text { WETLAND } & \square \text { LOCAL WETLAND }\end{array}$

URL: www.pinelandsnursery.com

\section{Cream Ridge}

Baier Lustgarten Farms \& Nurseries

107 Jonathan Holmes Rd.

Cream Ridge, NJ

08514

PHONE: (877) 493-7600

$\begin{array}{ll}\nabla \text { TREE } & \nabla \text { LOCAL TREE } \\ \nabla \text { SHRUB } & \nabla \text { LOCAL SHRUB } \\ \square \text { GRASS } & \square \text { LOCAL GRASS } \\ \square \text { FORBS } & \square \text { LOCAL FORBS } \\ \square \text { WETLAND } & \square \text { LOCAL WETLAND }\end{array}$

URL: none currently available 
Egg Harbor

Herb \& Botanical Alliance

POB Box 93

Egg Harbor, NJ

08215

PHONE: (609) 965-0337

$\begin{array}{ll}\square \text { TREE } & \square \text { LOCAL TREE } \\ \square \text { SHRUB } & \square \text { LOCAL SHRUB } \\ \square \text { GRASS } & \square \text { LOCAL GRASS } \\ \square \text { FORBS } & \square \text { LOCAL FORBS } \\ \square \text { WETLAND } & \square \text { LOCAL WETLAND }\end{array}$

URL: none currently available

\section{Farmingdale}

Jonathon Green Seeds

PO Box 326

Farmingdale, $\mathrm{NJ}$

07727

PHONE: (800) 243-0047

$\begin{array}{ll}\square \text { TREE } & \square \text { LOCAL TREE } \\ \square \text { SHRUB } & \square \text { LOCAL SHRUB } \\ \square \text { GRASS } & \square \text { LOCAL GRASS } \\ \square \text { FORBS } & \square \text { LOCAL FORBS } \\ \square \text { WETLAND } & \square \text { LOCAL WETLAND }\end{array}$

URL: www.jonathongreen.com

\section{Franklinville}

Triple Oaks Nursery \& Herb Garden

$\begin{array}{lll}\text { PO Box } 385 & \nabla \text { TREE } & \square \text { LOCAL TREE } \\ \text { Franklinville, NJ } & \nabla \text { SHRUB } & \square \text { LOCAL SHRUB } \\ \text { 08322 } & \square \text { GRASS } & \square \text { LOCAL GRASS } \\ \text { PHONE: (856) 694-4272 } & \square \text { FORBS } & \square \text { LOCAL FORBS } \\ & \square \text { WETLAND } & \square \text { LOCAL WETLAND }\end{array}$

URL: www.tripleoaks.com

\section{Freehold}

Wild Earth Native Plant Nursery

$\begin{array}{lll}49 \text { Mead Ave. } & \square \text { TREE } & \square \text { LOCAL TREE } \\ \text { Freehold, NJ } & \nabla \text { SHRUB } & \square \text { LOCAL SHRUB } \\ 07728 & \nabla \text { GRASS } & \square \text { LOCAL GRASS } \\ \text { PHONE: (732) 308-9777 } & \nabla \text { FORBS } & \square \text { LOCAL FORBS } \\ & \square \text { WETLAND } & \square \text { LOCAL WETLAND }\end{array}$

URL: none currently available

\section{Frenchtown}

Toad Shade Wildflower Farm

53 Everittstown Road

Frenchtown, NJ

08825

PHONE: (908) 996-7500

$\begin{array}{ll}\square \text { TREE } & \square \text { LOCAL TREE } \\ \square \text { SHRUB } & \square \text { LOCAL SHRUB } \\ \square \text { GRASS } & \square \text { LOCAL GRASS } \\ \square \text { FORBS } & \square \text { LOCAL FORBS } \\ \square \text { WETLAND } & \square \text { LOCAL WETLAND }\end{array}$

URL: www.toadshade.com

\begin{tabular}{|c|c|c|}
\hline \multicolumn{3}{|l|}{ Jackson } \\
\hline \multicolumn{3}{|l|}{ New Jersey Forest Tree Nursery } \\
\hline 370 East Veterans Highway & $\checkmark$ TREE & $\checkmark$ LOCAL TREE \\
\hline Jackson, NJ & $\square$ SHRUB & $\square$ LOCAL SHRUB \\
\hline 08527 & $\square$ GRASS & $\square$ LOCAL GRASS \\
\hline PHONE: (732) 928-0029 & $\square$ FORBS & $\square$ LOCAL FORBS \\
\hline & $\checkmark$ WETLAND & $\checkmark$ LOCAL WETLAND \\
\hline
\end{tabular}

URL: http://www state.ni.us/dep/parksandforests/forest/nifs seedling nursery.html 
Thompson \& Morgan, Inc.

Box 1308

Jackson, NJ

08527

PHONE: (908) 363-2225

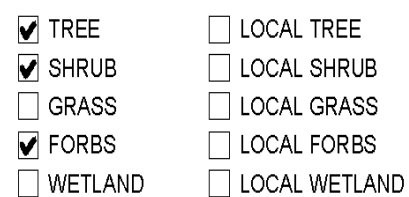

URL: http://seeds.thompson-morgan.com/us

\section{Kingston}

Mapleton Nurseries

PO Box 396

Kingston, NJ

08528

PHONE: (609) 430-0366

$\begin{array}{ll}\nabla \text { TREE } & \square \text { LOCAL TREE } \\ \nabla \text { SHRUB } & \square \text { LOCAL SHRUB } \\ \nabla \text { GRASS } & \square \text { LOCAL GRASS } \\ \nabla \text { FORBS } & \square \text { LOCAL FORBS } \\ \nabla \text { WETLAND } & \square \text { LOCAL WETLAND }\end{array}$

URL: none currently available

\section{Mauricetown}

Coastal Nurseries, Inc.

P.O. Box 42

Mauricetown, NJ

08329

PHONE: (609) 476-3903

$\begin{array}{ll}\nabla \text { TREE } & \square \text { LOCAL TREE } \\ \nabla \text { SHRUB } & \square \text { LOCAL SHRUB } \\ \nabla \text { GRASS } & \square \text { LOCAL GRASS } \\ \square \text { FORBS } & \square \text { LOCAL FORBS } \\ \nabla \text { WETLAND } & \nabla \text { LOCAL WETLAND }\end{array}$

URL: none currently available

Palermo

Philip Dubrielle

1433 South Shore Rd

Palermo, NJ

08230

PHONE: (609) 390-2058

$\begin{array}{ll}\square \text { TREE } & \square \text { LOCAL TREE } \\ \square \text { SHRUB } & \square \text { LOCAL SHRUB } \\ \square \text { GRASS } & \square \text { LOCAL GRASS } \\ \square \text { FORBS } & \square \text { LOCAL FORBS } \\ \square \text { WETLAND } & \square \text { LOCAL WETLAND }\end{array}$

URL: none currently available

Saddle River

Waterford Gardens

74 East Allendale Rd.

Saddle River, NJ

07458

PHONE: (201) 327-0721

$\begin{array}{ll}\square \text { TREE } & \square \text { LOCAL TREE } \\ \square \text { SHRUB } & \square \text { LOCAL SHRUB } \\ \square \text { GRASS } & \square \text { LOCAL GRASS } \\ \square \text { FORBS } & \square \text { LOCAL FORBS } \\ \square \text { WETLAND } & \square \text { LOCAL WETLAND }\end{array}$

URL: http:/Wwaterford-gardens.com/home.htm

\section{Williamstown}

Arrowwood Nursery Inc.

870 W. Malaga Rd.

Williamstown, $N\rfloor$

08094

PHONE: (609) 697-6045

$\begin{array}{ll}\nabla \text { TREE } & \square \text { LOCAL TREE } \\ \nabla \text { SHRUB } & \square \text { LOCAL SHRUB } \\ \square \text { GRASS } & \square \text { LOCAL GRASS } \\ \nabla \text { FORBS } & \nabla \text { LOCAL FORBS } \\ \square \text { WETLAND } & \square \text { LOCAL WETLAND }\end{array}$

URL: none currently available 


\section{New Mexico}

Albuquerque

Plants of the Southwest

6680 4th St. N.W.

Albuquerque, NM

87107

PHONE: (505) 344-8830

$\begin{array}{ll}\nabla \text { TREE } & \square \text { LOCAL TREE } \\ \nabla \text { SHRUB } & \square \text { LOCAL SHRUB } \\ \nabla \text { GRASS } & \square \text { LOCAL GRASS } \\ \nabla \text { FORBS } & \nabla \text { LOCAL FORBS } \\ \square \text { WETLAND } & \square \text { LOCAL WETLAND }\end{array}$

URL: www.plantsofthesouthwest.com

Plants World Inc.

250 El Pueblo Rd NE

Albuquerque, NM

$\checkmark$ TREE

$\checkmark$ SHRUB

$\nabla$ GRASS

PHONE: (505) 898-9627

$\nabla$ FORBS

$\checkmark$ LOCAL TREE

$\checkmark$ LOCAL SHRUB

URL: www.plantworldinc.com

$\square$ WETLAND

$\square$ LOCAL WETLAND

Seeds West Garden Seeds

31714 th St. N.W.

$\begin{array}{ll}\square \text { TREE } & \square \text { LOCAL TREE } \\ \square \text { SHRUB } & \square \text { LOCAL SHRUB } \\ \square \text { GRASS } & \square \text { LOCAL GRASS } \\ \nabla \text { FORBS } & \square \text { LOCAL FORBS } \\ \square \text { WETLAND } & \square \text { LOCAL WETLAND }\end{array}$

Albuquerque, NM

87104

PHONE: (505) 843-9713

$\square$ WETLAND

LOCAL WETLAND

URL: www.seedswestgardenseeds.com

\section{Alburquerque}

Natures Way Wholesale Nursery

8905 Edith NE

Alburquerque, NM

87113

PHONE: (800) 575-5329

$\begin{array}{ll}\nabla \text { TREE } & \square \text { LOCAL TREE } \\ \square \text { SHRUB } & \square \text { LOCAL SHRUB } \\ \nabla \text { GRASS } & \square \text { LOCAL GRASS } \\ \square \text { FORBS } & \square \text { LOCAL FORBS } \\ \nabla \text { WETLAND } & \square \text { LOCAL WETLAND }\end{array}$

URL: none currently available

\section{Belen}

Mesa Garden

P.O. Box 72

Belen, NM

87002

PHONE: (505) 864-3131

$\begin{array}{ll}\square \text { TREE } & \square \text { LOCAL TREE } \\ \square \text { SHRUB } & \square \text { LOCAL SHRUB } \\ \square \text { GRASS } & \square \text { LOCAL GRASS } \\ \square \text { FORBS } & \square \text { LOCAL FORBS } \\ \square \text { WETLAND } & \square \text { LOCAL WETLAND }\end{array}$

URL: none currently available

\section{Bernalillo}

Santa Ana Garden Center \& Native Plant Nursery

157 Jermez Dam Rd

Bernalillo, NM

87004

PHONE: (505) 867-1322

$\begin{array}{ll}\square \text { TREE } & \square \text { LOCAL TREE } \\ \square \text { SHRUB } & \square \text { LOCAL SHRUB } \\ \square \text { GRASS } & \square \text { LOCAL GRASS } \\ \square \text { FORBS } & \square \text { LOCAL FORBS } \\ \square \text { WETLAND } & \square \text { LOCAL WETLAND }\end{array}$

URL: none currently available 
Santa Ana Pueblo Native Plant Nursery

2 Dove Road

Bernalillo, NM

$\checkmark$ TREE $\square$ LOCAL TREE

87004

PHONE: (505) 867-1323

$\nabla$ SHRUB $\square$ LOCAL SHRUB

$\square$ GRASS $\square$ LOCAL GRASS

$\checkmark$ FORBS $\square$ LOCAL FORBS

$\checkmark$ WETLAND $\square$ LOCAL WETLAND

URL: none currently available

\section{Chamberino}

Sierra Vista Growers

P.O.Box 225

Chamberino, NM

88027

PHONE: (505) 874-2415

$\begin{array}{ll}\nabla \text { TREE } & \square \text { LOCAL TREE } \\ \nabla \text { SHRUB } & \square \text { LOCAL SHRUB } \\ \nabla \text { GRASS } & \square \text { LOCAL GRASS } \\ \nabla \text { FORBS } & \square \text { LOCAL FORBS } \\ \square \text { WETLAND } & \square \text { LOCAL WETLAND }\end{array}$

URL: none currently available

\section{Clovis}

Curtis \& Curtis, Inc.

Star Route Box $8 \mathrm{~A}$

Clovis, NM

88101

PHONE: (505) 762-4759

$\begin{array}{ll}\nabla \text { TREE } & \nabla \text { LOCAL TREE } \\ \nabla \text { SHRUB } & \square \text { LOCAL SHRUB } \\ \nabla \text { GRASS } & \nabla \text { LOCAL GRASS } \\ \nabla \text { FORBS } & \square \text { LOCAL FORBS } \\ \nabla \text { WETLAND } & \nabla \text { LOCAL WETLAND }\end{array}$

URL: www.curtisseed.com

\section{Las Cruces}

\section{Green Thumb Nursery}

2211 N. Mesquite St.

Las Cruces, NM

88001

PHONE: (505) 524-0592

URL: http://Www greenthumblc.com/

Plant Propagation Technologies, Inc. 250 S Crawford Blvd

Las Cruces, NM

88005

PHONE: (505) 527-9820

$\begin{array}{ll}\nabla \text { TREE } & \square \text { LOCAL TREE } \\ \square \text { SHRUB } & \square \text { LOCAL SHRUB } \\ \square \text { GRASS } & \square \text { LOCAL GRASS } \\ \square \text { FORBS } & \square \text { LOCAL FORBS } \\ \square \text { WETLAND } & \square \text { LOCAL WETLAND }\end{array}$

$\begin{array}{ll}\nabla \text { TREE } & \square \text { LOCAL TREE } \\ \nabla \text { SHRUB } & \square \text { LOCAL SHRUB } \\ \square \text { GRASS } & \square \text { LOCAL GRASS } \\ \square \text { FORBS } & \square \text { LOCAL FORBS } \\ \square \text { WETLAND } & \square \text { LOCAL WETLAND }\end{array}$

URL: none currently available

\section{Los Lunas}

Plant Materials Center

1036 Miller St. S.W

Los Lunas, NM

87031

PHONE: (505) 865-4684

$\begin{array}{ll}\square \text { TREE } & \square \text { LOCAL TREE } \\ \square \text { SHRUB } & \square \text { LOCAL SHRUB } \\ \square \text { GRASS } & \square \text { LOCAL GRASS } \\ \square \text { FORBS } & \square \text { LOCAL FORBS } \\ \square \text { WETLAND } & \square \text { LOCAL WETLAND }\end{array}$

URL: http://plant-materials.nrcs.usda.gov/nmpmc/ 
Trees That Please

9 Gilcrease Lane

Los Lunas, NM

87031

PHONE: (505) 866-5027

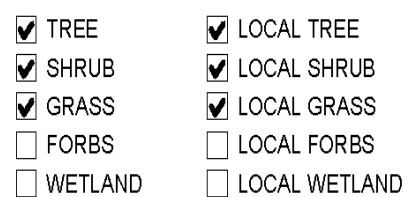

URL: none currently available

Roswell

Roswell Seed

P.O. Box 725

Roswell, NM

88202

PHONE: (505) 622-7701

$\begin{array}{ll}\square \text { TREE } & \square \text { LOCAL TREE } \\ \square \text { SHRUB } & \square \text { LOCAL SHRUB } \\ \square \text { GRASS } & \square \text { LOCAL GRASS } \\ \square \text { FORBS } & \square \text { LOCAL FORBS } \\ \square \text { WETLAND } & \square \text { LOCAL WETLAND }\end{array}$

URL: none currently available

\section{Santa Fe}

Agua Fria Nursery, Inc.

1409 Agua Fria St.

Santa Fe, NM

87501

PHONE: (505) 983-4831

$\begin{array}{ll}\nabla \text { TREE } & \square \text { LOCAL TREE } \\ \nabla \text { SHRUB } & \square \text { LOCAL SHRUB } \\ \square \text { GRASS } & \square \text { LOCAL GRASS } \\ \nabla \text { FORBS } & \square \text { LOCAL FORBS } \\ \square \text { WETLAND } & \square \text { LOCAL WETLAND }\end{array}$

URL: none currently available

Earthseeds

3369 Montezuma Ave. \#226

Santa Fe, NM

87501

PHONE: (505) 471-6926

$\begin{array}{ll}\square \text { TREE } & \square \text { LOCAL TREE } \\ \square \text { SHRUB } & \square \text { LOCAL SHRUB } \\ \nabla \text { GRASS } & \nabla \text { LOCAL GRASS } \\ \nabla \text { FORBS } & \nabla \text { LOCAL FORBS } \\ \square \text { WETLAND } & \square \text { LOCAL WETLAND }\end{array}$

URL: www.earthseed.com

\section{High Country Gardens}

2902 Rufina St.

$\square$ TREE

Santa $\mathrm{Fe}, \mathrm{NM}$

87505

PHONE: (800) 925-9387

URL: www.highcountrygardens.com

\section{New Mexico State Forestry}

P.O. Box 1948

$\checkmark$ TREE

$\nabla$ SHRUB

$\square$ LOCAL WETLAND

Santa Fe, NM

$\square$ GRASS

87504-194

PHONE: (505) 476-3353

\begin{tabular}{ll}
$\square$ FORBS & $\square$ LOCAL GRASS \\
$\square$ WETLAND & $\square$ LOCAL FORBS \\
\hline
\end{tabular}

URL: http://Www.emnrd. state.nm.us/FORESTRY//tree/web/home/main.cfm

Paynes Nurseries, Inc.

Box 4817

Santa Fe, NM

87502

PHONE: (505) 988-8011

$\begin{array}{ll}\nabla \text { TREE } & \square \text { LOCAL TREE } \\ \nabla \text { SHRUB } & \nabla \text { LOCAL SHRUB } \\ \nabla \text { GRASS } & \square \text { LOCAL GRASS } \\ \nabla \text { FORBS } & \nabla \text { LOCAL FORBS } \\ \square \text { WETLAND } & \square \text { LOCAL WETLAND }\end{array}$

URL: none currently available 
Plants of the Southwest Aqua Fria Rt. 6 Box 11-A

Santa Fe, NM

87501

PHONE: (505) 438-8888

$\begin{array}{ll}\nabla \text { TREE } & \nabla \text { LOCAL TREE } \\ \nabla \text { SHRUB } & \nabla \text { LOCAL SHRUB } \\ \nabla \text { GRASS } & \nabla \text { LOCAL GRASS } \\ \nabla \text { FORBS } & \nabla \text { LOCAL FORBS } \\ \square \text { WETLAND } & \square \text { LOCAL WETLAND }\end{array}$

URL: www.plantsofthesouthwest.com

Veguita

Desert Moon Nursery

Box 600

Veguita, NM

87062

PHONE: (505) 864-0614

$\begin{array}{ll}\nabla \text { TREE } & \boldsymbol{\nabla} \text { LOCAL TREE } \\ \nabla \text { SHRUB } & \boldsymbol{\nabla} \text { LOCAL SHRUB } \\ \square \text { GRASS } & \square \text { LOCAL GRASS } \\ \square \text { FORBS } & \square \text { LOCAL FORBS } \\ \square \text { WETLAND } & \square \text { LOCAL WETLAND }\end{array}$

URL: none currently available

\section{Nevada}

Blue Diamond

Blue Diamond Nursery

PO Box 268

Blue Diamond, NV

89004

PHONE: (702) 875-1968

$\begin{array}{ll}\square \text { TREE } & \square \text { LOCAL TREE } \\ \nabla \text { SHRUB } & \nabla \text { LOCAL SHRUB } \\ \square \text { GRASS } & \square \text { LOCAL GRASS } \\ \nabla \text { FORBS } & \square \text { LOCAL FORBS } \\ \square \text { WETLAND } & \square \text { LOCAL WETLAND }\end{array}$

URL: www.cactuscactus.com

\section{Boulder City}

Mojave Desert Nursery

P.O.Box 62066

Boulder City, NV

89006

PHONE: (702) 293-3940

$\begin{array}{ll}\nabla \text { TREE } & \square \text { LOCAL TREE } \\ \square \text { SHRUB } & \square \text { LOCAL SHRUB } \\ \square \text { GRASS } & \square \text { LOCAL GRASS } \\ \square \text { FORBS } & \square \text { LOCAL FORBS } \\ \square \text { WETLAND } & \square \text { LOCAL WETLAND }\end{array}$

URL: none currently available

\section{Carson City}

Nevada Division of Forestry - Washoe Nursery

885 Eastlake Blvd.

Carson City, NV

89704

PHONE: (775) 849-0213

$\begin{array}{ll}\nabla \text { TREE } & \boldsymbol{v} \text { LOCAL TREE } \\ \nabla \text { SHRUB } & \square \text { LOCAL SHRUB } \\ \square \text { GRASS } & \square \text { LOCAL GRASS } \\ \square \text { FORBS } & \square \text { LOCAL FORBS } \\ \square \text { WETLAND } & \square \text { LOCAL WETLAND }\end{array}$

URL: http://Www.forestry.nv.gov/main/nursery w.htm

\section{Las Vegas}

Nevada Division of Forestry Las Vegas Nursery 9600 Tule Springs Rd.

Las Vegas, NV

89131

PHONE: (702) 486-5411

$\begin{array}{ll}\nabla \text { TREE } & \square \text { LOCAL TREE } \\ \nabla \text { SHRUB } & \square \text { LOCAL SHRUB } \\ \square \text { GRASS } & \square \text { LOCAL GRASS } \\ \square \text { FORBS } & \square \text { LOCAL FORBS } \\ \square \text { WETLAND } & \square \text { LOCAL WETLAND }\end{array}$

URL: http://Www.forestry.nv.gov/main/nursey c.htm 
Reno

Comstock Seed

8520 W. 4th St.

Reno, NV

89523

$\square$ LOCAL TREE

PHONE: (702) 746-3681

$\checkmark$ SHRUB $\quad \nabla$ LOCAL SHRUB

$\nabla$ GRASS $\quad \nabla$ LOCAL GRASS

$\nabla$ FORBS $\quad \nabla$ LOCAL FORBS

URL: none currently available

Sparks

Albers of Nevada

2205 Glaendale Ave.

Sparks, NV

89431

$\square$ WETLAND $\square$ LOCAL WETLAND

PHONE: (702) 358-2077

$\begin{array}{ll}\square \text { TREE } & \square \text { LOCAL TREE } \\ \nabla \text { SHRUB } & \square \text { LOCAL SHRUB } \\ \nabla \text { GRASS } & \square \text { LOCAL GRASS } \\ \nabla \text { FORBS } & \square \text { LOCAL FORBS } \\ \square \text { WETLAND } & \square \text { LOCAL WETLAND }\end{array}$

URL: none currently available

\section{New York}

Baldwinsville

Merritt Seed Co.

7880 Gates Ro

Baldwinsville, NY

13027

PHONE: (315) 638-0610

$\begin{array}{ll}\square \text { TREE } & \square \text { LOCAL TREE } \\ \square \text { SHRUB } & \square \text { LOCAL SHRUB } \\ \square \text { GRASS } & \square \text { LOCAL GRASS } \\ \square \text { FORBS } & \square \text { LOCAL FORBS } \\ \square \text { WETLAND } & \square \text { LOCAL WETLAND }\end{array}$

URL: www.merrittseed.com

Buffalo

Stokes Seeds, Inc.

Box 548

$\square$ TREE

Buffalo, NY

14240

$\square$ SHRUB

$\square$ GRASS

LOCAL TREE

14240

$\checkmark$ FORBS

$\square$ LOCAL SHRUB

PHONE: (716) 695-6980

URL: www.stokeseeds.com

\section{Canandaigua}

J.E. Miller Nurseries, Inc.

5060 W. Lake Rd.

$\checkmark$ TREE

$\checkmark$ LOCAL TREE

Canandaigua, NY

14424

PHONE: (716) 396-2647

$\begin{array}{ll}\square \text { SHRUB } & \square \text { LOCAL SHRUB } \\ \square \text { GRASS } & \square \text { LOCAL GRASS } \\ \square \text { FORBS } & \square \text { LOCAL FORBS } \\ \square \text { WETLAND } & \square \text { LOCAL WETLAND }\end{array}$

URL: www.millemurseries.com

\section{Cooperstown}

Sylvan Botanicals American Ginseng

PO Box 91

Cooperstown, NY

13326

PHONE: (607) 264-8455

$\begin{array}{ll}\nabla \text { TREE } & \square \text { LOCAL TREE } \\ \square \text { SHRUB } & \square \text { LOCAL SHRUB } \\ \square \text { GRASS } & \square \text { LOCAL GRASS } \\ \nabla \text { FORBS } & \square \text { LOCAL FORBS } \\ \square \text { WETLAND } & \square \text { LOCAL WETLAND }\end{array}$

URL: www.catskillginseng.com 
Corning

Plant Materials Center

3266-A State Route 352

Corning. NY

14830

PHONE: (607) 562-8404

$\begin{array}{ll}\square \text { TREE } & \square \text { LOCAL TREE } \\ \square \text { SHRUB } & \square \text { LOCAL SHRUB } \\ \square \text { GRASS } & \square \text { LOCAL GRASS } \\ \square \text { FORBS } & \square \text { LOCAL FORBS } \\ \square \text { WETLAND } & \square \text { LOCAL WETLAND }\end{array}$

URL: http://plant-materials.nrcs.usda.gov/nypmc/

\section{Cutchogue}

Plantage, Inc.

Box 28

Cutchogue, NY

11935

PHONE: (516) 734-6832

$\begin{array}{ll}\square \text { TREE } & \square \text { LOCAL TREE } \\ \square \text { SHRUB } & \square \text { LOCAL SHRUB } \\ \square \text { GRASS } & \square \text { LOCAL GRASS } \\ \square \text { FORBS } & \square \text { LOCAL FORBS } \\ \square \text { WETLAND } & \square \text { LOCAL WETLAND }\end{array}$

URL: www.plantage.com

Dix Hills

Roslyn Nursery, Inc.

$\begin{array}{lll}211 \text { Burrs Ln } & \square \text { TREE } & \square \text { LOCAL TREE } \\ \text { Dix Hills, NY } & \square \text { SHRUB } & \square \text { LOCAL SHRUB } \\ 11746 & \square \text { GRASS } & \square \text { LOCAL GRASS } \\ \text { PHONE: (516) 643-9347 } & \square \text { FORBS } & \square \text { LOCAL FORBS } \\ & \square \text { WETLAND } & \square \text { LOCAL WETLAND }\end{array}$

URL: www.roslynnursery.com

Elma

Treehaven Evergreen Nursery

981 Jamison $\mathrm{Rd}$

Elma, NY

14059

$\boldsymbol{\nabla}$ TREE $\quad \boldsymbol{\nabla}$ LOCAL TREE

$\square$ SHRUB $\square$ LOCAL SHRUB

PHONE: (716) 652-4206

$\square$ GRASS $\square$ LOCAL GRASS

$\square$ FORBS $\quad \square$ LOCAL FORBS

$\square$ WETLAND $\square$ LOCAL WETLAND

URL: none currently available

Locke

Sheffields Tree \& Shrub Seed

273 Auburn Rd., Rt. 34"

Locke, NY

13092

PHONE: (315) 497-1058

$\begin{array}{ll}\nabla \text { TREE } & \square \text { LOCAL TREE } \\ \nabla \text { SHRUB } & \square \text { LOCAL SHRUB } \\ \nabla \text { GRASS } & \square \text { LOCAL GRASS } \\ \nabla \text { FORBS } & \square \text { LOCAL FORBS } \\ \square \text { WETLAND } & \square \text { LOCAL WETLAND }\end{array}$

URL: www.shefields.com

\section{North Collins}

Concord Nurseries, Inc. 10175 Mile Block Rd

North Collins, NY

14111

PHONE: (716) 337-2485

$\begin{array}{ll}\nabla \text { TREE } & \square \text { LOCAL TREE } \\ \nabla \text { SHRUB } & \square \text { LOCAL SHRUB } \\ \square \text { GRASS } & \square \text { LOCAL GRASS } \\ \square \text { FORBS } & \square \text { LOCAL FORBS } \\ \square \text { WETLAND } & \square \text { LOCAL WETLAND }\end{array}$

URL: www.concordnurseries.com 
Pawling

Native Landscaping Inc.

$\begin{array}{lll}\text { PO Box 327 } & \nabla \text { TREE } & \square \text { LOCAL TREE } \\ \text { Pawling, NY } & \square \text { SHRUB } & \square \text { LOCAL SHRUB } \\ 12564 & \nabla \text { GRASS } & \square \text { LOCAL GRASS } \\ \text { PHONE: (914) 855-7050 } & \square \text { FORBS } & \square \text { LOCAL FORBS } \\ & \square \text { WETLAND } & \square \text { LOCAL WETLAND }\end{array}$

URL: none currently available

\section{Potsdam}

St. Lawrence Nurseries

325 St. Hwy. 345, Potsdam-Madrid Rd.

Potsdam, NY

13676

PHONE: (315) 265-0778

$\begin{array}{ll}\nabla \text { TREE } & \square \text { LOCAL TREE } \\ \square \text { SHRUB } & \square \text { LOCAL SHRUB } \\ \square \text { GRASS } & \square \text { LOCAL GRASS } \\ \nabla \text { FORBS } & \nabla \text { LOCAL FORBS } \\ \square \text { WETLAND } & \square \text { LOCAL WETLAND }\end{array}$

URL: none currently available

Riverhead

Talmage Farm

2975 Sound Ave.

Riverhead, NY

11901

PHONE: (631) 727-0124

$\begin{array}{ll}\nabla \text { TREE } & \square \text { LOCAL TREE } \\ \nabla \text { SHRUB } & \square \text { LOCAL SHRUB } \\ \square \text { GRASS } & \square \text { LOCAL GRASS } \\ \square \text { FORBS } & \square \text { LOCAL FORBS } \\ \square \text { WETLAND } & \square \text { LOCAL WETLAND }\end{array}$

URL: wnw.talmagefarm.com

\section{Saratoga Springs}

NY State Dept. of Env. Cons. Saratoga Tree Nursery

2369 Route 50

Saratoga Springs, NY

12866

PHONE: (518) 581-1439

$\begin{array}{ll}\square \text { TREE } & \square \text { LOCAL TREE } \\ \square \text { SHRUB } & \square \text { LOCAL SHRUB } \\ \square \text { GRASS } & \square \text { LOCAL GRASS } \\ \square \text { FORBS } & \square \text { LOCAL FORBS } \\ \square \text { WETLAND } & \square \text { LOCAL WETLAND }\end{array}$

URL: http://WWw.dec.state.ny.us/website/dlf/privland/nursery/

\section{Springwater}

Amandas Garden

8410 Harpers Ferry Rd

Springwater, NY

14560

PHONE: (585) 669-2275

$\begin{array}{ll}\square \text { TREE } & \square \text { LOCAL TREE } \\ \square \text { SHRUB } & \square \text { LOCAL SHRUB } \\ \square \text { GRASS } & \square \text { LOCAL GRASS } \\ \square \text { FORBS } & \square \text { LOCAL FORBS } \\ \square \text { WETLAND } & \square \text { LOCAL WETLAND }\end{array}$

URL: none currently available

\section{West Clarksville}

Southern Tier Consulting, Inc.

PO Box 30

$\checkmark$ TREE

West Clarksville, NY

14786

PHONE: (716) 968-3120

URL: none currently available $\square$ SHRUB $\square$ LOCAL SHRUB

$\square$ GRASS $\square$ LOCAL GRASS

$\square$ FORBS $\quad \square$ LOCAL FORBS

$\checkmark$ WETLAND $\square$ LOCAL WETLAND 
West Hampton

Peat \& Son Nursery

32 Old Country Rd.

West Hampton, NY

11977

PHONE: (516) 288-3458

$\begin{array}{ll}\nabla \text { TREE } & \square \text { LOCAL TREE } \\ \nabla \text { SHRUB } & \square \text { LOCAL SHRUB } \\ \nabla \text { GRASS } & \square \text { LOCAL GRASS } \\ \nabla \text { FORBS } & \square \text { LOCAL FORBS } \\ \nabla \text { WETLAND } & \square \text { LOCAL WETLAND }\end{array}$

URL: none currently available

\section{Ohio}

Athens

Companion Plants

7247 N Coolville Ridge

Athens, $\mathrm{OH}$

45701

PHONE: (740) 592-4643

$\begin{array}{ll}\square \text { TREE } & \square \text { LOCAL TREE } \\ \square \text { SHRUB } & \square \text { LOCAL SHRUB } \\ \square \text { GRASS } & \square \text { LOCAL GRASS } \\ \square \text { FORBS } & \square \text { LOCAL FORBS } \\ \square \text { WETLAND } & \square \text { LOCAL WETLAND }\end{array}$

URL: http://Www.frognet.net/companion plants/

Batawa

B.C. Nursery

4183 SR 276

Batawa, $\mathrm{OH}$

45103

PHONE: (513) 724-9032

$\begin{array}{ll}\nabla \text { TREE } & \square \text { LOCAL TREE } \\ \nabla \text { SHRUB } & \square \text { LOCAL SHRUB } \\ \nabla \text { GRASS } & \square \text { LOCAL GRASS } \\ \square \text { FORBS } & \square \text { LOCAL FORBS } \\ \square \text { WETLAND } & \square \text { LOCAL WETLAND }\end{array}$

URL: none currently available

\section{Brecksville}

JMD Company

10324 Brecksville Rd.

Brecksville, $\mathrm{OH}$

44141

$\begin{array}{ll}\square \text { TREE } & \square \text { LOCAL TREE } \\ \square \text { SHRUB } & \square \text { LOCAL SHRUB } \\ \nabla \text { GRASS } & \square \text { LOCAL GRASS } \\ \square \text { FORBS } & \square \text { LOCAL FORBS } \\ \nabla \text { WETLAND } & \square \text { LOCAL WETLAND }\end{array}$

PHONE: (440) 526-0017

$\nabla$ WETLAND $\square$ LOCAL WETLAND

URL: www.imdcompany.com

Canfield

Green Valley Turf Farms Inc.

$\begin{array}{lll}\text { P.O. Box } 185 & \square \text { TREE } & \square \text { LOCAL TREE } \\ \text { Canfield, OH } & \square \text { SHRUB } & \square \text { LOCAL SHRUB } \\ \text { 44406 } & \square \text { GRASS } & \square \text { LOCAL GRASS } \\ \text { PHONE: (330) 533-3354 } & \square \text { FORBS } & \square \text { LOCAL FORBS } \\ & \square \text { WETLAND } & \square \text { LOCAL WETLAND }\end{array}$

URL: www.greenvalleyseed.com

\section{Hamilton}

Marys Plant Farm \& Landscape

2410 Lanes Mill Rd

Hamilton, $\mathrm{OH}$

45013

PHONE: (513) 894-0022

URL: none currently available

$\begin{array}{ll}\nabla \text { TREE } & \square \text { LOCAL TREE } \\ \nabla \text { SHRUB } & \square \text { LOCAL SHRUB } \\ \nabla \text { GRASS } & \square \text { LOCAL GRASS } \\ \nabla \text { FORBS } & \square \text { LOCAL FORBS } \\ \square \text { WETLAND } & \square \text { LOCAL WETLAND }\end{array}$

$\square$ WETLAND $\square$ LOCAL WETLAND 


\section{Independence}

William Tricker Inc.

7125 Tanglewood Dr.

Independence, $\mathrm{OH}$

44131

PHONE: (800) 524-3492

URL: www.tricker.com

$\begin{array}{ll}\square \text { TREE } & \square \text { LOCAL TREE } \\ \square \text { SHRUB } & \square \text { LOCAL SHRUB } \\ \square \text { GRASS } & \square \text { LOCAL GRASS } \\ \square \text { FORBS } & \square \text { LOCAL FORBS } \\ \square \text { WETLAND } & \square \text { LOCAL WETLAND }\end{array}$

North Lima

Mellingersinc.

2310 W. South Range Rd.

North Lima, $\mathrm{OH}$

44452

PHONE: (800) 321-7444

$\begin{array}{ll}\nabla \text { TREE } & \square \text { LOCAL TREE } \\ \square \text { SHRUB } & \square \text { LOCAL SHRUB } \\ \square \text { GRASS } & \square \text { LOCAL GRASS } \\ \square \text { FORBS } & \square \text { LOCAL FORBS } \\ \square \text { WETLAND } & \square \text { LOCAL WETLAND }\end{array}$

URL: www.mellingers.com

Ostrander

The Seed Center

7790 Marysville Rd

Ostrander, $\mathrm{OH}$

43061

PHONE: (740) 666-4050

$\begin{array}{ll}\square \text { TREE } & \square \text { LOCAL TREE } \\ \square \text { SHRUB } & \square \text { LOCAL SHRUB } \\ \square \text { GRASS } & \square \text { LOCAL GRASS } \\ \square \text { FORBS } & \square \text { LOCAL FORBS } \\ \square \text { WETLAND } & \square \text { LOCAL WETLAND }\end{array}$

URL: none currently available

\section{Perry}

Klyn Nurseries, Inc.

3322 S. Ridge Rd.

Perry, $\mathrm{OH}$

44081

PHONE: (440) 259-3811

$\begin{array}{ll}\square \text { TREE } & \square \text { LOCAL TREE } \\ \square \text { SHRUB } & \square \text { LOCAL SHRUB } \\ \square \text { GRASS } & \square \text { LOCAL GRASS } \\ \square \text { FORBS } & \square \text { LOCAL FORBS } \\ \square \text { WETLAND } & \square \text { LOCAL WETLAND }\end{array}$

URL: www.klynnurseries.com

Lake County Nursery Exchange

P.O. Box 122, Rt.84"

Perry, $\mathrm{OH}$

44081

PHONE: (440) 259-5571

$\begin{array}{ll}\nabla \text { TREE } & \square \text { LOCAL TREE } \\ \nabla \text { SHRUB } & \square \text { LOCAL SHRUB } \\ \square \text { GRASS } & \square \text { LOCAL GRASS } \\ \square \text { FORBS } & \square \text { LOCAL FORBS } \\ \square \text { WETLAND } & \square \text { LOCAL WETLAND }\end{array}$

URL: www.lakecountynursery.com

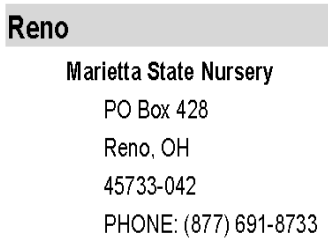

$\begin{array}{ll}\square \text { TREE } & \square \text { LOCAL TREE } \\ \square \text { SHRUB } & \square \text { LOCAL SHRUB } \\ \square \text { GRASS } & \square \text { LOCAL GRASS } \\ \square \text { FORBS } & \square \text { LOCAL FORBS } \\ \square \text { WETLAND } & \square \text { LOCAL WETLAND }\end{array}$

URL: http://Www.dnr.state.oh.us/forestry/Seedling/default.htm 
Rutland

Land Reforms Nursery \& Landscape

35703 Loop Rd

Rutland, $\mathrm{OH}$

45775

PHONE: (740) 742-3478

$\begin{array}{ll}\nabla \text { TREE } & \square \text { LOCAL TREE } \\ \nabla \text { SHRUB } & \square \text { LOCAL SHRUB } \\ \nabla \text { GRASS } & \square \text { LOCAL GRASS } \\ \nabla \text { FORBS } & \square \text { LOCAL FORBS } \\ \nabla \text { WETLAND } & \square \text { LOCAL WETLAND }\end{array}$

URL: none currently available

Solon

Cattail Meadows, Ltd

P.O.Box 39391

Solon, $\mathrm{OH}$

44139

PHONE: (440) 248-4581

$\begin{array}{ll}\square \text { SHRUB } & \square \text { LOCAL SHRUB } \\ \square \text { GRASS } & \square \text { LOCAL GRASS } \\ \nabla \text { FORBS } & \square \text { LOCAL FORBS } \\ \square \text { WETLAND } & \square \text { LOCAL WETLAND }\end{array}$

URL: none currently available

Somerset

Envirotech Consultants / Nursery

5380 Twp. Rd. 143 NE

Somerset, $\mathrm{OH}$

43783

PHONE: (740) 743-1669

$\begin{array}{ll}\square \text { TREE } & \square \text { LOCAL TREE } \\ \square \text { SHRUB } & \square \text { LOCAL SHRUB } \\ \nabla \text { GRASS } & \square \text { LOCAL GRASS } \\ \square \text { FORBS } & \square \text { LOCAL FORBS } \\ \square \text { WETLAND } & \square \text { LOCAL WETLAND }\end{array}$

URL: http:/Wwww.envirotechcon.com/

\section{Tipp City}

Saunders Seed Co.

P.O. Box 98

Tipp City, $\mathrm{OH}$

45371

PHONE: (937) 667-2313

$\begin{array}{ll}\square \text { TREE } & \square \text { LOCAL TREE } \\ \square \text { SHRUB } & \square \text { LOCAL SHRUB } \\ \square \text { GRASS } & \square \text { LOCAL GRASS } \\ \square \text { FORBS } & \square \text { LOCAL FORBS } \\ \square \text { WETLAND } & \square \text { LOCAL WETLAND }\end{array}$

URL: none currently available

Wauseon

Rupp Seed Co.

17919 County Rd. B

Wauseon, $\mathrm{OH}$

4356 ?

PHONE: (419) 445-1831

$\begin{array}{ll}\square \text { TREE } & \square \text { LOCAL TREE } \\ \square \text { SHRUB } & \square \text { LOCAL SHRUB } \\ \square \text { GRASS } & \square \text { LOCAL GRASS } \\ \square \text { FORBS } & \square \text { LOCAL FORBS } \\ \square \text { WETLAND } & \square \text { LOCAL WETLAND }\end{array}$

URL: wWw.ruppseeds.com

Whitehall

JMD Company

4637 Poth Rd, Unit A

Whitehall, $\mathrm{OH}$

43213

$\begin{array}{ll}\square \text { TREE } & \square \text { LOCAL TREE } \\ \square \text { SHRUB } & \square \text { LOCAL SHRUB } \\ \square \text { GRASS } & \square \text { LOCAL GRASS } \\ \square \text { FORBS } & \square \text { LOCAL FORBS } \\ \square \text { WETLAND } & \square \text { LOCAL WETLAND }\end{array}$

PHONE: (614) 866-0452

$\checkmark$ WETLAND $\square$ LOCAL WETLAND

URL: www.mdcompany.com 
Wilmington

Lofts Seed, Inc.

Box 888

Wilmington, $\mathrm{OH}$

45177

PHONE: (800) 328-1127

$\begin{array}{ll}\square \text { TREE } & \square \text { LOCAL TREE } \\ \square \text { SHRUB } & \square \text { LOCAL SHRUB } \\ \square \text { GRASS } & \square \text { LOCAL GRASS } \\ \square \text { FORBS } & \square \text { LOCAL FORBS } \\ \square \text { WETLAND } & \square \text { LOCAL WETLAND }\end{array}$

URL: www.turf.com

\section{Oklahoma}

Bixby

John Deere Landscapes

Box 752

$\nabla$ TREE

Bixby, OK

74008

$\checkmark$ SHRUB

$\checkmark$ GRASS

TREE

PHONE: (918) 369-2091

$\checkmark$ FORBS

$\square$ LOCAL GRASS

$\square$ WETLAND

$\square$ LOCAL FORBS

$\checkmark$ LOCAL WETLAND

URL: $\underline{\text { www.johndeerelandscapes.com }}$

\section{Enid}

Johnston Seed Co.

Box 1392

Enid, OK

73702

PHONE: (800) 375-4613

$\begin{array}{ll}\square \text { TREE } & \square \text { LOCAL TREE } \\ \square \text { SHRUB } & \square \text { LOCAL SHRUB } \\ \nabla \text { GRASS } & \boldsymbol{\nabla} \text { LOCAL GRASS } \\ \square \text { FORBS } & \square \text { LOCAL FORBS } \\ \square \text { WETLAND } & \square \text { LOCAL WETLAND }\end{array}$

URL: www.johnstonseed.com

\section{Hennessey}

Grasslander

Rt.1, Box $56^{\prime \prime}$

Hennessey, OK

73742

PHONE: (405) 853-2607

$\begin{array}{ll}\square \text { TREE } & \square \text { LOCAL TREE } \\ \square \text { SHRUB } & \square \text { LOCAL SHRUB } \\ \nabla \text { GRASS } & \square \text { LOCAL GRASS } \\ \square \text { FORBS } & \square \text { LOCAL FORBS } \\ \nabla \text { WETLAND } & \square \text { LOCAL WETLAND }\end{array}$

URL: none currently available

Okeene

Lorenzos OK Seeds

$101 \mathrm{~N}$. Main

$\square$ TREE

Okeene, OK

73763

PHONE: (405) 822-3655

$\begin{array}{ll}\square \text { SHRUB } & \square \text { LOCAL SHRUB } \\ \nabla \text { GRASS } & \nabla \text { LOCAL GRASS } \\ \nabla \text { FORBS } & \nabla \text { LOCAL FORBS } \\ \nabla \text { WETLAND } & \nabla \text { LOCAL WETLAND }\end{array}$

URL: none currently available

\section{Oklahoma City}

Eckroat Seed, Inc.

Box 17610

Oklahoma City, OK

73136

PHONE: (405) 427-2484

$\begin{array}{ll}\square \text { TREE } & \square \text { LOCAL TREE } \\ \square \text { SHRUB } & \square \text { LOCAL SHRUB } \\ \square \text { GRASS } & \square \text { LOCAL GRASS } \\ \square \text { FORBS } & \square \text { LOCAL FORBS } \\ \square \text { WETLAND } & \square \text { LOCAL WETLAND }\end{array}$

URL: www.eckroatseed.com 
Melots

8900 W. Memorial Rd.

Oklahoma City, OK

73142

PHONE: (405) 721-4394

$\begin{array}{ll}\square \text { TREE } & \square \text { LOCAL TREE } \\ \square \text { SHRUB } & \square \text { LOCAL SHRUB } \\ \square \text { GRASS } & \square \text { LOCAL GRASS } \\ \square \text { FORBS } & \square \text { LOCAL FORBS } \\ \square \text { WETLAND } & \square \text { LOCAL WETLAND }\end{array}$

URL: none currently available

Park Hill

Greenleaf Nursery

28406 Hwy 82

Park Hill, OK

74457

PHONE: (918) 457-5172

$\begin{array}{ll}\nabla \text { TREE } & \square \text { LOCAL TREE } \\ \nabla \text { SHRUB } & \square \text { LOCAL SHRUB } \\ \nabla \text { GRASS } & \square \text { LOCAL GRASS } \\ \nabla \text { FORBS } & \square \text { LOCAL FORBS } \\ \square \text { WETLAND } & \square \text { LOCAL WETLAND }\end{array}$

URL: www.greenleafnursery.com

Perry

Brorsen Bluestems

Rt. 1. Box 199A"

Perry, OK

73077

PHONE: (580) 336-4963

$\begin{array}{ll}\square \text { TREE } & \square \text { LOCAL TREE } \\ \square \text { SHRUB } & \square \text { LOCAL SHRUB } \\ \square \text { GRASS } & \square \text { LOCAL GRASS } \\ \square \text { FORBS } & \square \text { LOCAL FORBS } \\ \square \text { WETLAND } & \square \text { LOCAL WETLAND }\end{array}$

URL: none currently available

Tahlequah

Tri-B Nursery

PO Box 436

Tahlequah, OK

74465

PHONE: (800) 244-6157

$\begin{array}{ll}\nabla \text { TREE } & \square \text { LOCAL TREE } \\ \square \text { SHRUB } & \square \text { LOCAL SHRUB } \\ \square \text { GRASS } & \square \text { LOCAL GRASS } \\ \square \text { FORBS } & \square \text { LOCAL FORBS } \\ \square \text { WETLAND } & \square \text { LOCAL WETLAND }\end{array}$

URL: none currently available

Washington

Oklahoma State Forest Nursery

Route 1, Box 44"

Washington, OK

73093

PHONE: (405) 288-2385

$\begin{array}{ll}\square \text { TREE } & \square \text { LOCAL TREE } \\ \square \text { SHRUB } & \square \text { LOCAL SHRUB } \\ \square \text { GRASS } & \square \text { LOCAL GRASS } \\ \square \text { FORBS } & \square \text { LOCAL FORBS } \\ \square \text { WETLAND } & \square \text { LOCAL WETLAND }\end{array}$

URL: http:/Wwww. state.ok.us/ okag/index.htm

\section{Oregon}

Albany

Seven Oaks Native Nursery

29730 Harvest Dr SW

Albany, $O R$

97321

PHONE: (541) 757-6520

URL: none currently available

$\begin{array}{ll}\nabla \text { TREE } & \square \text { LOCAL TREE } \\ \nabla \text { SHRUB } & \square \text { LOCAL SHRUB } \\ \nabla \text { GRASS } & \square \text { LOCAL GRASS } \\ \nabla \text { FORBS } & \square \text { LOCAL FORBS } \\ \square \text { WETLAND } & \square \text { LOCAL WETLAND }\end{array}$

$\checkmark$ TREE

$\square$ GRASS

$\checkmark$ WETLAND

$\square$ LOCAL WETLAND 
Aurora

Samuel J. Rich Nursery 9803 Yergen Rd. NE

Aurora, OR

97002

PHONE: (503) 678-2828

URL: none currently available

Weyerhaeuser; Aurora Forest Nursery

6051 S Lone Elder Rd

Aurora, OR

97002

PHONE: (503) 266-2018

$\begin{array}{ll}\nabla \text { TREE } & \square \text { LOCAL TREE } \\ \nabla \text { SHRUB } & \square \text { LOCAL SHRUB } \\ \square \text { GRASS } & \square \text { LOCAL GRASS } \\ \square \text { FORBS } & \square \text { LOCAL FORBS } \\ \square \text { WETLAND } & \square \text { LOCAL WETLAND }\end{array}$

URL: http://Nww.weyerhaeuser.com/

$\begin{array}{ll}\square \text { TREE } & \square \text { LOCAL TREE } \\ \square \text { SHRUB } & \square \text { LOCAL SHRUB } \\ \square \text { GRASS } & \square \text { LOCAL GRASS } \\ \square \text { FORBS } & \square \text { LOCAL FORBS } \\ \square \text { WETLAND } & \checkmark \text { LOCAL WETLAND }\end{array}$

Banks

Wind Hill Native Gardens

PO Box 789

Banks, OR

TREE $\square$ LOCAL TREE

97106

PHONE: (503) 324-6817

$\checkmark$ SHRUB

$\checkmark$ LOCAL SHRUB

$\square$ GRASS

$\checkmark$ FORBS

$\square$ LOCAL GRASS

$\checkmark$ WETLAND $\square$ LOCAL WETLAND

URL: none currently available

Beaver

Green Hills Nursery

40805 Upper Nestucca Rd.

Beaver, $O R$

97108

PHONE: (503) 398-5965

$\begin{array}{ll}\nabla \text { TREE } & \square \text { LOCAL TREE } \\ \square \text { SHRUB } & \square \text { LOCAL SHRUB } \\ \square \text { GRASS } & \square \text { LOCAL GRASS } \\ \square \text { FORBS } & \square \text { LOCAL FORBS } \\ \square \text { WETLAND } & \square \text { LOCAL WETLAND }\end{array}$

URL: none currently available

Boring

J. Frank Schmidt \& Son Co.

P.O. Box 189

Boring, $O R$

97009

PHONE: (503) 663-4128

$\begin{array}{ll}\nabla \text { TREE } & \square \text { LOCAL TREE } \\ \nabla \text { SHRUB } & \square \text { LOCAL SHRUB } \\ \square \text { GRASS } & \square \text { LOCAL GRASS } \\ \square \text { FORBS } & \square \text { LOCAL FORBS } \\ \square \text { WETLAND } & \square \text { LOCAL WETLAND }\end{array}$

URL: www.ffschmidt.com

Plants Unlimited

PO Box 26

Boring, $O R$

97009

PHONE: (503) 663-5267

$\begin{array}{ll}\nabla \text { TREE } & \square \text { LOCAL TREE } \\ \nabla \text { SHRUB } & \square \text { LOCAL SHRUB } \\ \nabla \text { GRASS } & \square \text { LOCAL GRASS } \\ \square \text { FORBS } & \square \text { LOCAL FORBS } \\ \square \text { WETLAND } & \square \text { LOCAL WETLAND }\end{array}$

URL: www.plantsunlimited.com 
Canby

A. McGill \& Son

Box 967

Canby, OR

97013

PHONE: (503) 651-2346

$\begin{array}{ll}\checkmark \text { TREE } & \checkmark \text { LOCAL TREE } \\ \square \text { SHRUB } & \square \text { LOCAL SHRUB } \\ \square \text { GRASS } & \square \text { LOCAL GRASS } \\ \square \text { FORBS } & \square \text { LOCAL FORBS } \\ \square \text { WETLAND } & \square \text { LOCAL WETLAND }\end{array}$

URL: none currently available

Adams-Franklin Tree Seedlings Nursery

1155 S Grant St

Canby, $O R$

97013

PHONE: (503) 266-1748

$\begin{array}{ll}\nabla \text { TREE } & \square \text { LOCAL TREE } \\ \square \text { SHRUB } & \square \text { LOCAL SHRUB } \\ \square \text { GRASS } & \square \text { LOCAL GRASS } \\ \square \text { FORBS } & \square \text { LOCAL FORBS } \\ \square \text { WETLAND } & \square \text { LOCAL WETLAND }\end{array}$

URL: none currently available

Douglas Nurseries

P.O. Box 47

$\checkmark$ TREE $\quad \nabla$ LOCAL TREE

Canby, $O R$

97013

PHONE: (503) 266-9419

$\checkmark$ SHRUB

$\checkmark$ LOCAL SHRUB

$\square$ GRASS

$\square$ LOCAL GRASS

$\square$ FORBS $\quad \square$ LOCAL FORBS

$\square$ WETLAND $\square$ LOCAL WETLAND

URL: www.douglasnursery.com

Four Mile Nursery

27027 S. Hwy. 170

Canby, $O R$

97013

PHONE: (503) 266-9572

$\begin{array}{ll}\nabla \text { TREE } & \square \text { LOCAL TREE } \\ \square \text { SHRUB } & \square \text { LOCAL SHRUB } \\ \square \text { GRASS } & \square \text { LOCAL GRASS } \\ \square \text { FORBS } & \square \text { LOCAL FORBS } \\ \square \text { WETLAND } & \square \text { LOCAL WETLAND }\end{array}$

URL: www.fourmile.com

\section{Hazels Country Gardens}

23230 S Hwy $99 \mathrm{E}$

Canby, $O R$

97013

PHONE: (503) 266-4071

$\begin{array}{ll}\nabla \text { TREE } & \square \text { LOCAL TREE } \\ \nabla \text { SHRUB } & \square \text { LOCAL SHRUB } \\ \square \text { GRASS } & \square \text { LOCAL GRASS } \\ \square \text { FORBS } & \square \text { LOCAL FORBS } \\ \square \text { WETLAND } & \square \text { LOCAL WETLAND }\end{array}$

URL: none currently available

IFA Nurseries, Inc.

136 NE Territorial Rd

Canby, OR

97013

PHONE: (503) 266-1940

$\begin{array}{ll}\nabla \text { TREE } & \nabla \text { LOCAL TREE } \\ \square \text { SHRUB } & \square \text { LOCAL SHRUB } \\ \square \text { GRASS } & \square \text { LOCAL GRASS } \\ \square \text { FORBS } & \square \text { LOCAL FORBS } \\ \square \text { WETLAND } & \square \text { LOCAL WETLAND }\end{array}$

URL: http://Mww ifanurseries.com/index.cfm

\section{Wichita Nursery}

9413 S Heinz Rd

Canby, OR

97013

PHONE: (503) 651-2279

$\begin{array}{ll}\nabla \text { TREE } & \square \text { LOCAL TREE } \\ \nabla \text { SHRUB } & \square \text { LOCAL SHRUB } \\ \nabla \text { GRASS } & \square \text { LOCAL GRASS } \\ \nabla \text { FORBS } & \square \text { LOCAL FORBS } \\ \nabla \text { WETLAND } & \square \text { LOCAL WETLAND }\end{array}$

URL: www.wichitanursery.com 
Cave Junction

Althouse Nursery

5410 Dick George Rd.

Cave Junction, $O R$

97523

PHONE: (541) 592-2395

$\begin{array}{ll}\nabla \text { TREE } & \square \text { LOCAL TREE } \\ \nabla \text { SHRUB } & \square \text { LOCAL SHRUB } \\ \square \text { GRASS } & \square \text { LOCAL GRASS } \\ \square \text { FORBS } & \square \text { LOCAL FORBS } \\ \square \text { WETLAND } & \square \text { LOCAL WETLAND }\end{array}$

URL: none currently available

\section{Corvallis}

Plant Materials Center

3415 N.E. Granger Ave

Corvallis, $O R$

97330

PHONE: (541) 757-4812

$\begin{array}{ll}\square \text { TREE } & \square \text { LOCAL TREE } \\ \square \text { SHRUB } & \square \text { LOCAL SHRUB } \\ \square \text { GRASS } & \square \text { LOCAL GRASS } \\ \square \text { FORBS } & \square \text { LOCAL FORBS } \\ \square \text { WETLAND } & \square \text { LOCAL WETLAND }\end{array}$

URL: http://plant-materials.nrcs.usda.cov/orpmc/

Dayton

Carlton Plants $\mathrm{C}_{0}$.

P.O. Box 398

Dayton, OR

97114

PHONE: (503) 868-7971

$\begin{array}{ll}\square \text { TREE } & \square \text { LOCAL TREE } \\ \square \text { SHRUB } & \square \text { LOCAL SHRUB } \\ \square \text { GRASS } & \square \text { LOCAL GRASS } \\ \square \text { FORBS } & \square \text { LOCAL FORBS } \\ \square \text { WETLAND } & \square \text { LOCAL WETLAND }\end{array}$

URL: none currently available

\section{Elkton}

D.L. Phipps Forest Nursery

2424 Wells Rd

Elkton, $\mathrm{OR}$

97436

PHONE: (541) 584-2214

URL: http://Www.odf.state.or.us/nursery.home.htm

\section{Eugene}

Greer Gardens

1280 Goodpasture Island Rd

Eugene, $O R$

97401

PHONE: (541) 686-8266

URL: www.greergardens.com

Irwin \& Sons Ag-Supply Inc.

$4209 \mathrm{~W}$. 6th Ave

Eugene, $O R$

97402

PHONE: (541) 345-1887

URL: none currently available

$\begin{array}{ll}\square \text { TREE } & \square \text { LOCAL TREE } \\ \square \text { SHRUB } & \square \text { LOCAL SHRUB } \\ \square \text { GRASS } & \square \text { LOCAL GRASS } \\ \square \text { FORBS } & \square \text { LOCAL FORBS } \\ \square \text { WETLAND } & \square \text { LOCAL WETLAND }\end{array}$

$\checkmark$ WETLAND 
Jules Bubacz

85432 Teague Loop

Eugene, $O R$

97405

PHONE: (541) 683-4416

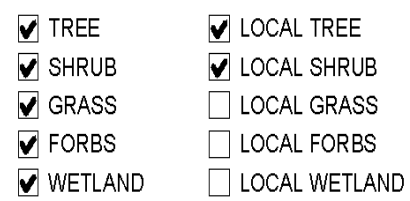

URL: none currently available

Florence

Wave Beachgrass Nursery

Box 1190

Florence, OR

97439

PHONE: (541) 997-2401

$\square$ LOCAL TREE

$\square$ SHRUB $\quad \square$ LOCAL SHRUB

$\nabla$ GRASS $\square$ LOCAL GRASS

$\square$ FORBS $\square$ LOCAL FORBS

$\checkmark$ WETLAND $\square$ LOCAL WETLAND

URL: none currently available

Woodsman Native Nursery

4385 Hwy. 101

$\square$ TREE $\quad \square$ LOCAL TREE

Florence, OR

97439

PHONE: (541) 997-2252

$\checkmark$ SHRUB

$\checkmark$ LOCAL SHRUB

$\square$ GRASS

$\square$ LOCAL GRASS

$\checkmark$ FORBS

$\checkmark$ LOCAL FORBS

$\square$ WETLAND $\square$ LOCAL WETLAND

URL: none currently available

Forest Grove

E.F. Nursery

43465 S.W. Hiatt

Forest Grove, OR

97116

PHONE: (503) 357-7157

$\checkmark$ TREE $\quad \boldsymbol{\nabla}$ LOCAL TREE

$\square$ SHRUB $\square$ LOCAL SHRUB

$\square$ GRASS $\square$ LOCAL GRASS

$\square$ FORBS $\square$ LOCAL FORBS

$\square$ WETLAND $\square$ LOCAL WETLAND

URL: www.efnursery.com

Flora Lan Nursery Inc.

7940 NW Kansas City Rd

Forest Grove, OR

97116

PHONE: (503) 357-8386

$\begin{array}{ll}\square \text { TREE } & \square \text { LOCAL TREE } \\ \square \text { SHRUB } & \square \text { LOCAL SHRUB } \\ \square \text { GRASS } & \square \text { LOCAL GRASS } \\ \square \text { FORBS } & \square \text { LOCAL FORBS } \\ \square \text { WETLAND } & \square \text { LOCAL WETLAND }\end{array}$

URL: none currently available

\section{Gresham}

Sus andales Pond \& Bog Plants

23665 SE Borges Rd

Gresham, OR

97080

PHONE: (503) 661-4259

$\begin{array}{ll}\square \text { TREE } & \square \text { LOCAL TREE } \\ \square \text { SHRUB } & \square \text { LOCAL SHRUB } \\ \square \text { GRASS } & \square \text { LOCAL GRASS } \\ \square \text { FORBS } & \square \text { LOCAL FORBS } \\ \square \text { WETLAND } & \square \text { LOCAL WETLAND }\end{array}$

URL: none currently available 
Halsey

DLF Jenks

Box 168

Halsey, OR

97348

PHONE: (541) 369-2251

URL: www.dlfusa.com

$\begin{array}{ll}\square \text { TREE } & \square \text { LOCAL TREE } \\ \square \text { SHRUB } & \square \text { LOCAL SHRUB } \\ \square \text { GRASS } & \square \text { LOCAL GRASS } \\ \square \text { FORBS } & \square \text { LOCAL FORBS } \\ \square \text { WETLAND } & \square \text { LOCAL WETLAND }\end{array}$

Hillsboro

Big Trees Today, Inc.

PO Box 1402

Hillsboro, $\mathrm{OR}$

97123

PHONE: (503) 640-3011

$\begin{array}{ll}\nabla \text { TREE } & \nabla \text { LOCAL TREE } \\ \nabla \text { SHRUB } & \checkmark \text { LOCAL SHRUB } \\ \square \text { GRASS } & \square \text { LOCAL GRASS } \\ \square \text { FORBS } & \square \text { LOCAL FORBS } \\ \square \text { WETLAND } & \square \text { LOCAL WETLAND }\end{array}$

URL: www.bigtreestoday.com

Cascadian Nurseries, Inc. 8900 NW Dick Rd

Hillsboro, $\mathrm{OR}$

97124

PHONE: (503) 647-9292

$\begin{array}{ll}\nabla \text { TREE } & \nabla \text { LOCAL TREE } \\ \nabla \text { SHRUB } & \nabla \text { LOCAL SHRUB } \\ \square \text { GRASS } & \square \text { LOCAL GRASS } \\ \square \text { FORBS } & \square \text { LOCAL FORBS } \\ \square \text { WETLAND } & \square \text { LOCAL WETLAND }\end{array}$

URL: www.cascadiannurseries.com

Hubbard

D. Wells Farms

P.O. Box 336

Hubbard, $\mathrm{OR}$

97037

PHONE: (503) 982-1012

$\begin{array}{ll}\nabla \text { TREE } & \square \text { LOCAL TREE } \\ \square \text { SHRUB } & \nabla \text { LOCAL SHRUB } \\ \square \text { GRASS } & \square \text { LOCAL GRASS } \\ \square \text { FORBS } & \square \text { LOCAL FORBS } \\ \square \text { WETLAND } & \square \text { LOCAL WETLAND }\end{array}$

URL: www.dwellsfarms.com

Turf-Seed

Box 250

Hubbard, $O R$

97032

PHONE: (602) 488-6050

$\begin{array}{ll}\square \text { TREE } & \square \text { LOCAL TREE } \\ \square \text { SHRUB } & \square \text { LOCAL SHRUB } \\ \square \text { GRASS } & \square \text { LOCAL GRASS } \\ \square \text { FORBS } & \square \text { LOCAL FORBS } \\ \square \text { WETLAND } & \square \text { LOCAL WETLAND }\end{array}$

URL: www.turf-seed.com

Junction City

Forbes Seed \& Grain, Inc.

Box 85

Junction City, OR

97448

PHONE: (541) 998-8086

$\begin{array}{ll}\square \text { TREE } & \square \text { LOCAL TREE } \\ \square \text { SHRUB } & \square \text { LOCAL SHRUB } \\ \square \text { GRASS } & \square \text { LOCAL GRASS } \\ \square \text { FORBS } & \square \text { LOCAL FORBS } \\ \square \text { WETLAND } & \square \text { LOCAL WETLAND }\end{array}$

URL: www.forbesseed.com 


\section{Klamath Falls}

$D$ \& D Seed

7343 S. 6th St.

Klamath Falls, OR

97603

PHONE: (541) 882-7799

URL: www.danddseeds.com

$\begin{array}{ll}\square \text { TREE } & \square \text { LOCAL TREE } \\ \square \text { SHRUB } & \square \text { LOCAL SHRUB } \\ \square \text { GRASS } & \square \text { LOCAL GRASS } \\ \square \text { FORBS } & \square \text { LOCAL FORBS } \\ \square \text { WETLAND } & \square \text { LOCAL WETLAND }\end{array}$

$\square$ WETLAND $\square$ LOCAL WETLAND

\section{La Grande}

The Plantworks

1805 U Ave

La Grande, OR

97850

PHONE: (541) 963-7870

$\begin{array}{ll}\nabla \text { TREE } & \nabla \text { LOCAL TREE } \\ \nabla \text { SHRUB } & \nabla \text { LOCAL SHRUB } \\ \nabla \text { GRASS } & \nabla \text { LOCAL GRASS } \\ \nabla \text { FORBS } & \nabla \text { LOCAL FORBS } \\ \nabla \text { WETLAND } & \nabla \text { LOCAL WETLAND }\end{array}$

URL: none currently available

\section{Lebanon}

La Ferme Noire

39621 Almen Drive

Lebanon, $\mathrm{OR}$

97355

PHONE: (541) 258-8990

URL: none currently available

Lorane

Balance Restoration Nursery

27995 Chambers Mill Rd.

Lorane, $\mathrm{OR}$

97451

PHONE: (541) 942-5530

$\begin{array}{ll}\nabla \text { TREE } & \square \text { LOCAL TREE } \\ \nabla \text { SHRUB } & \nabla \text { LOCAL SHRUB } \\ \nabla \text { GRASS } & \nabla \text { LOCAL GRASS } \\ \square \text { FORBS } & \square \text { LOCAL FORBS } \\ \nabla \text { WETLAND } & \square \text { LOCAL WETLAND }\end{array}$

$\begin{array}{ll}\nabla \text { TREE } & \checkmark \text { LOCAL TREE } \\ \nabla \text { SHRUB } & \checkmark \text { LOCAL SHRUB } \\ \nabla \text { GRASS } & \checkmark \text { LOCAL GRASS } \\ \nabla \text { FORBS } & \nabla \text { LOCAL FORBS } \\ \nabla \text { WETLAND } & \checkmark \text { LOCAL WETLAND }\end{array}$

URL: none currently available

Merrill

Highland Seed \& Supply

P.O. Box 553

Merrill, $\mathrm{OR}$

97633

PHONE: (541) 798-5235

$\begin{array}{ll}\square \text { TREE } & \square \text { LOCAL TREE } \\ \square \text { SHRUB } & \square \text { LOCAL SHRUB } \\ \square \text { GRASS } & \square \text { LOCAL GRASS } \\ \square \text { FORBS } & \square \text { LOCAL FORBS } \\ \square \text { WETLAND } & \square \text { LOCAL WETLAND }\end{array}$

URL: none currently available

Molalla

Quail Ridge Nursery

33689 S. Ball Rd.

Molalla, OR

97038

PHONE: (503) 829-3105

$\begin{array}{ll}\nabla \text { TREE } & \square \text { LOCAL TREE } \\ \nabla \text { SHRUB } & \nabla \text { LOCAL SHRUB } \\ \square \text { GRASS } & \square \text { LOCAL GRASS } \\ \square \text { FORBS } & \square \text { LOCAL FORBS } \\ \square \text { WETLAND } & \square \text { LOCAL WETLAND }\end{array}$

URL: none currently available 
Mt. Angel

Barth Nursery

8456 May Road NE

Mt. Angel, $O R$

97362

PHONE: (503) 845-6955

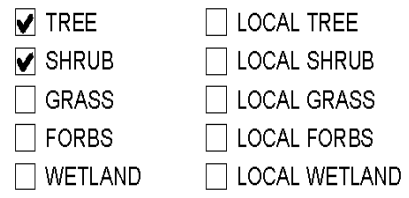

URL: none currently available

Newberg

Wine Country Nursery \& Aquarium

4100 NE Portland Rd

Newberg, OR

97172

PHONE: (503) 538-1518

$\begin{array}{ll}\nabla \text { TREE } & \square \text { LOCAL TREE } \\ \square \text { SHRUB } & \square \text { LOCAL SHRUB } \\ \square \text { GRASS } & \square \text { LOCAL GRASS } \\ \square \text { FORBS } & \square \text { LOCAL FORBS } \\ \square \text { WETLAND } & \square \text { LOCAL WETLAND }\end{array}$

URL: none currently available

Newport

Ferris Nursery

P.O. Box 258

Newport, $O R$

97365

PHONE: (541) 265-5709

$\begin{array}{ll}\nabla \text { TREE } & \square \text { LOCAL TREE } \\ \nabla \text { SHRUB } & \square \text { LOCAL SHRUB } \\ \nabla \text { GRASS } & \square \text { LOCAL GRASS } \\ \nabla \text { FORBS } & \square \text { LOCAL FORBS } \\ \nabla \text { WETLAND } & \square \text { LOCAL WETLAND }\end{array}$

URL: none currently available

North Bend

Huckleberry Lane Nursery

PO Box 316

North Bend, $O R$

97459

PHONE: (541) 756-7328

$\begin{array}{ll}\square \text { TREE } & \square \text { LOCAL TREE } \\ \nabla \text { SHRUB } & \checkmark \text { LOCAL SHRUB } \\ \square \text { GRASS } & \square \text { LOCAL GRASS } \\ \square \text { FORBS } & \square \text { LOCAL FORBS } \\ \square \text { WETLAND } & \square \text { LOCAL WETLAND }\end{array}$

URL: none currently available

\section{Ontario}

Andrews Seed Co.

580 S. Oregon St.

Ontario, $\mathrm{OR}$

97309

PHONE: (541) 889-9109

$\begin{array}{ll}\square \text { TREE } & \square \text { LOCAL TREE } \\ \square \text { SHRUB } & \square \text { LOCAL SHRUB } \\ \square \text { GRASS } & \square \text { LOCAL GRASS } \\ \square \text { FORBS } & \square \text { LOCAL FORBS } \\ \square \text { WETLAND } & \square \text { LOCAL WETLAND }\end{array}$

URL: none currently available

\section{Philomath}

Wild Garden Seed

PO Box 1509

Philomath, $\mathrm{OR}$

97370

PHONE: (541) 929-4068

$\begin{array}{ll}\square \text { TREE } & \square \text { LOCAL TREE } \\ \square \text { SHRUB } & \square \text { LOCAL SHRUB } \\ \square \text { GRASS } & \square \text { LOCAL GRASS } \\ \square \text { FORBS } & \square \text { LOCAL FORBS } \\ \square \text { WETLAND } & \square \text { LOCAL WETLAND }\end{array}$

URL: none currently available 
Port Orford

Curry Native Plants

92545 Silver Butte Rd

Port Orford, OR

97465

PHONE: (541) 332-5635

$\begin{array}{ll}\nabla \text { TREE } & \square \text { LOCAL TREE } \\ \nabla \text { SHRUB } & \square \text { LOCAL SHRUB } \\ \square \text { GRASS } & \square \text { LOCAL GRASS } \\ \square \text { FORBS } & \square \text { LOCAL FORBS } \\ \square \text { WETLAND } & \square \text { LOCAL WETLAND }\end{array}$

URL: none currently available

\section{Portland}

Hobbs \& Hopkins LTD.

1712 SE Ankeny St.

Portland, $O R$

97214

PHONE: (503) 239-7518

$\begin{array}{ll}\square \text { SHRUB } & \square \text { LOCAL SHRUB } \\ \square \text { GRASS } & \square \text { LOCAL GRASS } \\ \square \text { FORBS } & \square \text { LOCAL FORBS } \\ \square \text { WETLAND } & \square \text { LOCAL WETLAND }\end{array}$

URL: http:/Www.protimelawnseed.com/

Landiseed International, Ltd.

P.O. Box 25690

$\begin{array}{ll}\square \text { TREE } & \square \text { LOCAL TREE } \\ \square \text { SHRUB } & \square \text { LOCAL SHRUB } \\ \square \text { GRASS } & \square \text { LOCAL GRASS } \\ \square \text { FORBS } & \square \text { LOCAL FORBS } \\ \square \text { WETLAND } & \square \text { LOCAL WETLAND }\end{array}$

Porlland,

97298

PHONE: (503) 203-6956

$\square$ WETLAND $\square$ LOCAL WETLAND

URL: none currently available

Portland Nursery
5050 SE Stark
Portland, OR
97215
PHONE: (503) 231-5050

URL: none currently available

\section{Sauvie Island Ornamentals}

19708 NW Gilihan Rd

Portland, OR

97231

PHONE: (503) 621-3086

URL: none currently available

Sunmark Seeds International 503 NW Irving St., Ste 200A

Portland, OR

97204

PHONE: (503) 241-7333

URL: www.sunmarkseeds.com

Teufel Nursery, Inc.

12345 N.W. Barns Rd.

Portland, $O R$

97229

PHONE: (800) 483-8335

URL: www.teufel.com

$\begin{array}{ll}\nabla \text { TREE } & \square \text { LOCAL TREE } \\ \nabla \text { SHRUB } & \square \text { LOCAL SHRUB } \\ \square \text { GRASS } & \square \text { LOCAL GRASS } \\ \square \text { FORBS } & \square \text { LOCAL FORBS } \\ \square \text { WETLAND } & \square \text { LOCAL WETLAND }\end{array}$

$\nabla$ TREE

$\square$ SHRUB

$\square$ GRASS

$\square$ FORBS

$\square$ WETLAND

$\checkmark$ LOCAL TREE

$\square$ LOCAL SHRUB

$\square$ LOCAL GRASS

$\square$ LOCAL FORBS

$\square$ LOCAL WETLAND

$\begin{array}{ll}\square \text { TREE } & \square \text { LOCAL TREE } \\ \nabla \text { SHRUB } & \square \text { LOCAL SHRUB } \\ \nabla \text { GRASS } & \square \text { LOCAL GRASS } \\ \square \text { FORBS } & \square \text { LOCAL FORBS } \\ \nabla \text { WETLAND } & \square \text { LOCAL WETLAND }\end{array}$

$\checkmark$ WETLAND $\square$ LOCAL WETLAND

$\begin{array}{ll}\nabla \text { TREE } & \square \text { LOCAL TREE } \\ \nabla \text { SHRUB } & \square \text { LOCAL SHRUB } \\ \nabla \text { GRASS } & \square \text { LOCAL GRASS } \\ \nabla \text { FORBS } & \square \text { LOCAL FORBS } \\ \square \text { WETLAND } & \square \text { LOCAL WETLAND }\end{array}$




\begin{tabular}{|c|c|c|}
\hline \multicolumn{3}{|l|}{ Salem } \\
\hline \multicolumn{3}{|l|}{ Alpha Nursery Inc. } \\
\hline 5050 Hazel Green Rd NE & $\nabla$ TREE & $\checkmark$ LOCAL TREE \\
\hline Salem, OR & $\checkmark$ SHRUB & $\nabla$ LOCAL SHRUB \\
\hline 97305 & $\checkmark$ GRASS & $\square$ LOCAL GRASS \\
\hline \multirow[t]{2}{*}{ PHONE: (503) 390-1286 } & $\nabla$ FORBS & $\square$ LOCAL FORBS \\
\hline & $\square$ WETLAND & $\square$ LOCAL WETLAND \\
\hline \multicolumn{3}{|l|}{ URL: www.alphanursery.com } \\
\hline \multicolumn{3}{|l|}{ Bailey Seed Co., Inc. } \\
\hline PO Box 13517 & $\square$ TREE & $\square$ LOCAL TREE \\
\hline Salem, OR & $\square$ SHRUB & $\square$ LOCAL SHRUB \\
\hline 97302 & $\nabla$ GRASS & $\square$ LOCAL GRASS \\
\hline \multirow[t]{2}{*}{ PHONE: $(800) 407-7713$} & $\checkmark$ FORBS & $\square$ LOCAL FORBS \\
\hline & $\square$ WETLAND & $\square$ LOCAL WETLAND \\
\hline \multicolumn{3}{|l|}{ URL: www.baileyseed.com } \\
\hline \multicolumn{3}{|l|}{ Brooks Tree Farm } \\
\hline 9785 Portland Rd. N.E. & $\nabla$ TREE & $\nabla$ LOCAL TREE \\
\hline Salem, OR & $\checkmark$ SHRUB & $\checkmark$ LOCAL SHRUB \\
\hline 97305 & $\square$ GRASS & $\square$ LOCAL GRASS \\
\hline \multirow[t]{2}{*}{ PHONE: (503) 393-6300 } & $\square$ FORBS & $\square$ LOCAL FORBS \\
\hline & $\checkmark$ WETLAND & $\checkmark$ LOCAL WETLAND \\
\hline \multicolumn{3}{|l|}{ URL: www.brookstreefarm.com } \\
\hline \multicolumn{3}{|l|}{ Cousins Nursery } \\
\hline 5144 36th Ave SE & $\nabla$ TREE & $\square$ LOCAL TREE \\
\hline Salem, OR & $\nabla$ SHRUB & $\square$ LOCAL SHRUB \\
\hline 97301 & $\square$ GRASS & $\square$ LOCAL GRASS \\
\hline \multirow{2}{*}{ PHONE: (503) 364-1240 } & $\square$ FORBS & $\square$ LOCAL FORBS \\
\hline & $\square$ WETLAND & $\square$ LOCAL WETLAND \\
\hline \multicolumn{3}{|l|}{ URL: none currently available } \\
\hline \multicolumn{3}{|l|}{ Heritage Seedlings, Inc. } \\
\hline 4199 75th Ave, SE, Suite 275 & $\checkmark$ TREE & $\checkmark$ LOCAL TREE \\
\hline Salem, OR & $\nabla$ SHRUB & $\nabla$ LOCAL SHRUB \\
\hline 97301 & $\nabla$ GRASS & $\checkmark$ LOCAL GRASS \\
\hline \multirow[t]{2}{*}{ PHONE: (541) 504-7269 } & $\checkmark$ FORBS & $\nabla$ LOCAL FORBS \\
\hline & $\checkmark$ WETLAND & $\nabla$ LOCAL WETLAND \\
\hline \multicolumn{3}{|c|}{ URL: www,heritageseedlings.com } \\
\hline \multicolumn{3}{|c|}{ Mt. Jefferson Farms, Inc. } \\
\hline PO Box 12708 & $\nabla$ TREE & $\nabla$ LOCAL TREE \\
\hline Salem, OR & $\checkmark$ SHRUB & $\nabla$ LOCAL SHRUB \\
\hline 97309 & $\nabla$ GRASS & $\nabla$ LOCAL GRASS \\
\hline \multirow[t]{2}{*}{ PHONE: (503) 363-0467 } & $\square$ FORBS & $\square$ LOCAL FORBS \\
\hline & $\checkmark$ WETLAND & $\nabla$ LOCAL WETLAND \\
\hline \multicolumn{3}{|l|}{ URL: none currently available } \\
\hline \multicolumn{3}{|l|}{ Russell Graham Purveyor of Plants } \\
\hline 4030 Eagle Crest Rd., N.W." & $\square$ TREE & $\square$ LOCAL TREE \\
\hline Salem, OR & $\square$ SHRUB & $\square$ LOCAL SHRUB \\
\hline 97304 & $\square$ GRASS & $\square$ LOCAL GRASS \\
\hline \multirow[t]{2}{*}{ PHONE: (503) 362-1135 } & $\bar{\nabla}$ FORBS & $\bar{\nabla}$ LOCAL FORBS \\
\hline & $\square$ WETLAND & $\square$ LOCAL WETLAND \\
\hline
\end{tabular}

URL: none currently available 
Whitman Farms

3995 Gibson Rd. NW

$\nabla$ TREE $\quad \nabla$ LOCAL TREE

Salem, OR

$\nabla$ SHRUB $\quad \nabla$ LOCAL SHRUB

97304

$\square$ GRASS $\square$ LOCAL GRASS

PHONE: (503) 585-8728

$\square$ FORBS $\square$ LOCAL FORBS

$\square$ WETLAND $\square$ LOCAL WETLAND

URL: www.whitmanfarms.com

Sandy

Alpine Hollow Tree Farm

PO Box 464

Sandy, $O R$

97055

PHONE: (503) 669-5245

$\checkmark$ TREE $\quad \nabla$ LOCAL TREE

$\nabla$ SHRUB $\nabla$ LOCAL SHRUB

$\square$ GRASS $\square$ LOCAL GRASS

$\square$ FORBS $\square$ LOCAL FORBS

$\square$ WETLAND $\square$ LOCAL WETLAND

URL: none currently available

Scio

Natures Garden

40611 Highway 226

Scio, OR

$\square$ TREE

$\nabla$ SHRUB

$\square$ LOCAL TREE

97374

PHONE: (503) 394-3217

$\square$ LOCAL GRASS

$\square$ WETLAND $\square$ LOCAL WETLAND

URL: none currently available

\section{Silverton}

Drakes Crossing Nursery

19774 Grade Rd. S.E.

Silverton, $\mathrm{OR}$

97381

PHONE: (503) 873-4932

$\begin{array}{ll}\nabla \text { TREE } & \square \text { LOCAL TREE } \\ \square \text { SHRUB } & \square \text { LOCAL SHRUB } \\ \square \text { GRASS } & \square \text { LOCAL GRASS } \\ \square \text { FORBS } & \square \text { LOCAL FORBS } \\ \square \text { WETLAND } & \square \text { LOCAL WETLAND }\end{array}$

URL: none currently available

Springfield

Log Cabin Nursery

3820 Hayden Bridge Rd

Springfield, $O R$

97477

PHONE: (541) 747-4324

$\begin{array}{ll}\square \text { TREE } & \square \text { LOCAL TREE } \\ \square \text { SHRUB } & \square \text { LOCAL SHRUB } \\ \square \text { GRASS } & \square \text { LOCAL GRASS } \\ \square \text { FORBS } & \square \text { LOCAL FORBS } \\ \square \text { WETLAND } & \square \text { LOCAL WETLAND }\end{array}$

URL: none currently available

St. Paul

Western Breeze Nursery

3838 Blanchet Ave NE

St. Paul, OR

97137

PHONE: (503) 633-4491

$\begin{array}{ll}\square \text { TREE } & \square \text { LOCAL TREE } \\ \square \text { SHRUB } & \square \text { LOCAL SHRUB } \\ \square \text { GRASS } & \square \text { LOCAL GRASS } \\ \square \text { FORBS } & \square \text { LOCAL FORBS } \\ \square \text { WETLAND } & \square \text { LOCAL WETLAND }\end{array}$

URL: none currently available 
Talent

English Heritage Gardens

851 Anderson Creek Rd

Talent, OR

97540

PHONE: (541) 535-1761

$\begin{array}{ll}\square \text { TREE } & \square \text { LOCAL TREE } \\ \square \text { SHRUB } & \square \text { LOCAL SHRUB } \\ \square \text { GRASS } & \square \text { LOCAL GRASS } \\ \square \text { FORBS } & \square \text { LOCAL FORBS } \\ \square \text { WETLAND } & \square \text { LOCAL WETLAND }\end{array}$

URL: none currently available

Plant Oregon

8677 Wagner Creek Rd

Talent, OR

97540

PHONE: (541) 535-3531

$\begin{array}{ll}\nabla \text { TREE } & \square \text { LOCAL TREE } \\ \nabla \text { SHRUB } & \square \text { LOCAL SHRUB } \\ \nabla \text { GRASS } & \square \text { LOCAL GRASS } \\ \nabla \text { FORBS } & \square \text { LOCAL FORBS } \\ \nabla \text { WETLAND } & \square \text { LOCAL WETLAND }\end{array}$

URL: www.plantoregon.com

Tangent

CHS

33731 Highway $99 \mathrm{E}$

Tangent, $O R$

97389

PHONE: (541) 928-2393

$\begin{array}{ll}\square \text { TREE } & \square \text { LOCAL TREE } \\ \square \text { SHRUB } & \square \text { LOCAL SHRUB } \\ \square \text { GRASS } & \square \text { LOCAL GRASS } \\ \square \text { FORBS } & \square \text { LOCAL FORBS } \\ \square \text { WETLAND } & \square \text { LOCAL WETLAND }\end{array}$

URL: none currently available

Tualatin

Greenleaf Nursery

9335 S.W. Greenhill Lane

Tualatin, $O R$

97062

PHONE: (503) 692-3483

$\nabla$ TREE $\quad \nabla$ LOCAL TREE

$\checkmark$ SHRUB $\quad \nabla$ LOCAL SHRUB

$\square$ GRASS $\square$ LOCAL GRASS

$\square$ FORBS $\square$ LOCAL FORBS

$\square$ WETLAND $\square$ LOCAL WETLAND

URL: none currently available

Hughes Water Garden

25289 SW Stafford Rd.

Tualatin, $O R$

97062

PHONE: (800) 858-1709

$\begin{array}{ll}\square \text { TREE } & \square \text { LOCAL TREE } \\ \square \text { SHRUB } & \square \text { LOCAL SHRUB } \\ \square \text { GRASS } & \square \text { LOCAL GRASS } \\ \square \text { FORBS } & \square \text { LOCAL FORBS } \\ \square \text { WETLAND } & \square \text { LOCAL WETLAND }\end{array}$

URL: www.thewatergardenshop.com

Williams

Forest Farm Nursery

990 Tetherow Rd.

Williams, OR

97544

PHONE: (541) 846-7269

$\begin{array}{ll}\nabla \text { TREE } & \square \text { LOCAL TREE } \\ \nabla \text { SHRUB } & \square \text { LOCAL SHRUB } \\ \nabla \text { GRASS } & \square \text { LOCAL GRASS } \\ \nabla \text { FORBS } & \square \text { LOCAL FORBS } \\ \nabla \text { WETLAND } & \square \text { LOCAL WETLAND }\end{array}$

URL: www.forestfarm.com 
Goodwin Creek Gardens

PO Box 83

Williams, $O R$

97544

PHONE: (541) 846-7357

$\begin{array}{ll}\nabla \text { TREE } & \square \text { LOCAL TREE } \\ \square \text { SHRUB } & \square \text { LOCAL SHRUB } \\ \square \text { GRASS } & \square \text { LOCAL GRASS } \\ \square \text { FORBS } & \square \text { LOCAL FORBS } \\ \square \text { WETLAND } & \square \text { LOCAL WETLAND }\end{array}$

URL: www.goodwincreekgardens.com

Wilsonville

Alder View Natives

28315 SW Grahams Ferry Rd

Wilsonville, $\mathrm{OR}$

97070

PHONE: (503) 570-2894

$\checkmark$ TREE $\quad \nabla$ LOCAL TREE

$\nabla$ SHRUB $\nabla$ LOCAL SHRUB

GRASS LOCAL GRASS

$\square$ FORBS $\square$ LOCAL FORBS

$\checkmark$ WETLAND $\square$ LOCAL WETLAND

URL: none currently available

Eby Nursery, Inc.

PO Box 1127

Wilsonville, $\mathrm{OR}$

97070

PHONE: (503) 638-8222

$\checkmark$ TREE

$\checkmark$ SHRUB

$\square$ GRASS

$\checkmark$ FORBS

$\checkmark$ WETLAND $\square$ LOCAL TREE

$\square$ LOCAL SHRUB

$\square$ LOCAL GRASS

$\square$ LOCAL FORBS

$\square$ LOCAL WETLAND

URL: none currently available

Winston

Wildwood Nursery

8374 Old Hwy 99 S

Winston, OR

97486

PHONE: (541) 679-4006

$\begin{array}{ll}\square \text { TREE } & \square \text { LOCAL TREE } \\ \square \text { SHRUB } & \square \text { LOCAL SHRUB } \\ \square \text { GRASS } & \square \text { LOCAL GRASS } \\ \square \text { FORBS } & \square \text { LOCAL FORBS } \\ \square \text { WETLAND } & \square \text { LOCAL WETLAND }\end{array}$

URL: none currently available

\section{Pennsylvania}

Allentown

Green Tree Nurseries 5300 Crackersport Rd.

Allentown, PA

18104

PHONE: (610) 395-6777

$\begin{array}{ll}\square \text { TREE } & \square \text { LOCAL TREE } \\ \square \text { SHRUB } & \square \text { LOCAL SHRUB } \\ \square \text { GRASS } & \square \text { LOCAL GRASS } \\ \square \text { FORBS } & \square \text { LOCAL FORBS } \\ \square \text { WETLAND } & \square \text { LOCAL WETLAND }\end{array}$

URL: www.bigtreeman.com

Loft Seed Inc.

2202 Hangar PI.

Allentown, PA

18103

PHONE: (610) 266-6612

$\begin{array}{ll}\square \text { TREE } & \square \text { LOCAL TREE } \\ \square \text { SHRUB } & \square \text { LOCAL SHRUB } \\ \square \text { GRASS } & \square \text { LOCAL GRASS } \\ \square \text { FORBS } & \square \text { LOCAL FORBS } \\ \square \text { WETLAND } & \square \text { LOCAL WETLAND }\end{array}$

URL: www.turf.com 
Apollo

Wetland Supply Co./Native Plant Nursery

1633 Gilmar Rd

Apollo, PA

15613

$\checkmark$ LOCAL TREE

$\checkmark$ SHRUB $\quad \nabla$ LOCAL SHRUB

PHONE: (724) 327-1830

$\nabla$ GRASS $\square$ LOCAL GRASS

$\nabla$ FORBS $\quad \nabla$ LOCAL FORBS

$\nabla$ WETLAND $\square$ LOCAL WETLAND

URL: none currently available

\section{Bethel Park}

JMD Company

5401 Progress Blvd.

Bethel Park, PA

15102

PHONE: (412) 833-7100

$\begin{array}{ll}\square \text { TREE } & \square \text { LOCAL TREE } \\ \square \text { SHRUB } & \square \text { LOCAL SHRUB } \\ \square \text { GRASS } & \square \text { LOCAL GRASS } \\ \square \text { FORBS } & \square \text { LOCAL FORBS } \\ \square \text { WETLAND } & \square \text { LOCAL WETLAND }\end{array}$

URL: www.mdcompany.com

\section{Chadds Ford}

Brandywine Conservancy

PO Box 141

Chadds Ford, PA

19317

PHONE: (610) 388-2700

$\begin{array}{ll}\square \text { TREE } & \square \text { LOCAL TREE } \\ \square \text { SHRUB } & \square \text { LOCAL SHRUB } \\ \square \text { GRASS } & \square \text { LOCAL GRASS } \\ \square \text { FORBS } & \square \text { LOCAL FORBS } \\ \square \text { WETLAND } & \square \text { LOCAL WETLAND }\end{array}$

URL: www.brandwwinemuseum.org

\section{Clearfield}

Pine Grove Nursery

RD 3 Box 146

Clearfield, $P A$

16830

PHONE: (814) 765-2363

$\begin{array}{ll}\nabla \text { TREE } & \square \text { LOCAL TREE } \\ \nabla \text { SHRUB } & \checkmark \text { LOCAL SHRUB } \\ \square \text { GRASS } & \square \text { LOCAL GRASS } \\ \square \text { FORBS } & \square \text { LOCAL FORBS } \\ \square \text { WETLAND } & \square \text { LOCAL WETLAND }\end{array}$

URL: none currently available

Delta

Doyle Farm Nursery

158 Norris Road

Delta, PA

17314

PHONE: (717) 862-3134

$\begin{array}{ll}\square \text { TREE } & \square \text { LOCAL TREE } \\ \square \text { SHRUB } & \square \text { LOCAL SHRUB } \\ \nabla \text { GRASS } & \square \text { LOCAL GRASS } \\ \nabla \text { FORBS } & \square \text { LOCAL FORBS } \\ \square \text { WETLAND } & \square \text { LOCAL WETLAND }\end{array}$

URL: none currently available

\begin{tabular}{|c|c|c|}
\hline Dover & & \\
\hline Strathmeyer Forest, Inc. & & \\
\hline 255 Zeigler Rd. & $\checkmark$ TREE & $\checkmark$ LOCAL TREE \\
\hline Dover, PA & $\square$ SHRUB & $\square$ LOCAL SHRUB \\
\hline 17315 & $\square$ GRASS & $\square$ LOCAL GRASS \\
\hline PHONE: (717) 292-5683 & $\square$ FORBS & $\square$ LOCAL FORBS \\
\hline & $\square$ WETLAND & $\square$ LOCAL WETLAND \\
\hline
\end{tabular}

URL: www.strathmever.com 
Drifting

Zetts Fish Farm \& Hatcheries

RR1, Box 405

Drifing, PA

16834

PHONE: (814) 345-5357

$\square$ SHRUB $\square$ LOCAL SHRUB

$\square$ GRASS $\square$ LOCAL GRASS

$\square$ FORBS $\square$ LOCAL FORBS

$\checkmark$ WETLAND $\square$ LOCAL WETLAND

URL: none currently available

Fairview

Fairview Evergreen Nurseries

PO Box E

$\nabla$ TREE $\quad \nabla$ LOCAL TREE

Fairview, PA

$\checkmark$ SHRUB

$\checkmark$ LOCAL SHRUB

16415

$\square$ GRASS

$\square$ LOCAL GRASS

PHONE: (814) 474-5712

$\square$ FORBS $\square$ LOCAL FORBS

$\square$ WETLAND $\square$ LOCAL WETLAND

URL: unw.fairviewevergreen.com

Glen Mills

Redbud Native Plant Nursery, LLC

1214 N Middletown Rd

Glen Mills, PA

19342

PHONE: (610) 358-4300

$\checkmark$ TREE $\quad \nabla$ LOCAL TREE

$\checkmark$ SHRUB $\nabla$ LOCAL SHRUB

$\nabla$ GRASS $\quad \nabla$ LOCAL GRASS

$\checkmark$ FORBS $\quad \nabla$ LOCAL FORBS

$\checkmark$ WETLAND $\square$ LOCAL WETLAND

URL: www.redbudnativeplantnursery.com

Glen Rock

Sylva Native Nursery \& Seed Co.

3815 Roser Rd

$\nabla$ TREE $\quad \nabla$ LOCAL TREE

Glen Rock, PA

$\checkmark$ SHRUB

$\checkmark$ LOCAL SHRUB

17327

$\checkmark$ GRASS

$\checkmark$ LOCAL GRASS

PHONE: (717) 227-0486

$\square$ FORBS $\square$ LOCAL FORBS

$\checkmark$ WETLAND $\square$ LOCAL WETLAND

URL: WWw.sylvanative.com

Harleysville

Sandy Wilson Native Plant \& Aquatic DBA: Sanwil Inc.

834 Church Rd.

Harleysville, PA

19438

$\checkmark$ SHRUB $\quad \checkmark$ LOCAL SHRUB

$\checkmark$ GRASS $\square$ LOCAL GRASS

PHONE: (610) 584-6302

$\checkmark$ FORBS $\square$ LOCAL FORBS

$\nabla$ WETLAND $\nabla$ LOCAL WETLAND

URL: none currently available

\section{Holbrook}

Pettit Seed Sales

RD 1

Holbrook, PA

15341

PHONE: (724) 499-5228

$\begin{array}{ll}\square \text { TREE } & \square \text { LOCAL TREE } \\ \square \text { SHRUB } & \square \text { LOCAL SHRUB } \\ \square \text { GRASS } & \square \text { LOCAL GRASS } \\ \square \text { FORBS } & \square \text { LOCAL FORBS } \\ \square \text { WETLAND } & \square \text { LOCAL WETLAND }\end{array}$

URL: none currently available 
Honey Brook

Englands Herb Farm

33 Todd Rd

Honey Brook, PA

19344

PHONE: (610) 273-2863

URL: none currently available

$\begin{array}{ll}\square \text { TREE } & \square \text { LOCAL TREE } \\ \square \text { SHRUB } & \square \text { LOCAL SHRUB } \\ \nabla \text { GRASS } & \square \text { LOCAL GRASS } \\ \nabla \text { FORBS } & \square \text { LOCAL FORBS } \\ \nabla \text { WETLAND } & \square \text { LOCAL WETLAND }\end{array}$

$\nabla$ WETLAND $\nabla$ LOCAL WETLAND

$\begin{array}{lll}\text { Horsham } & & \\ \text { Seeds, Inc. } & & \\ 307 \text { Horsham Rd. } & \square \text { TREE } & \square \text { LOCAL TREE } \\ \text { Horsham, PA } & \square \text { SHRUB } & \square \text { LOCAL SHRUB } \\ 19044 & \square \text { GRASS } & \square \text { LOCAL GRASS } \\ \text { PHONE: (215) 675-2186 } & \square \text { FORBS } & \square \text { LOCAL FORBS } \\ & \square \text { WETLAND } & \square \text { LOCAL WETLAND }\end{array}$

URL: none currently available

Indiana

Carino Nurseries

Box 538

Indiana, PA

15701

PHONE: (724) 463-3350

URL: www.carinonurseries.com

Musser Forests inc

1880 Hwy N

Indiana, PA

15701

PHONE: (724) 465-5685

URL: wWw.musserforests.com

$\begin{array}{ll}\nabla \text { TREE } & \square \text { LOCAL TREE } \\ \nabla \text { SHRUB } & \checkmark \text { LOCAL SHRUB } \\ \square \text { GRASS } & \square \text { LOCAL GRASS } \\ \square \text { FORBS } & \square \text { LOCAL FORBS } \\ \square \text { WETLAND } & \square \text { LOCAL WETLAND }\end{array}$

$\checkmark$ TREE

$\square$ SHRUB

$\checkmark$ LOCAL TREE

$\square$ LOCAL SHRUB

$\square$ LOCAL GRASS

$\begin{array}{ll}\square \text { FORBS } & \square \text { LOCAL FORBS } \\ \square \text { WETLAND } & \square \text { LOCAL WETLAND }\end{array}$

\section{od}

Octararo Native Plant Nursery

6126 Street Rd.

Kirkwood, PA

17536

PHONE: (717) 529-3160

$\begin{array}{ll}\nabla \text { TREE } & \square \text { LOCAL TREE } \\ \nabla \text { SHRUB } & \square \text { LOCAL SHRUB } \\ \square \text { GRASS } & \square \text { LOCAL GRASS } \\ \square \text { FORBS } & \square \text { LOCAL FORBS } \\ \nabla \text { WETLAND } & \square \text { LOCAL WETLAND }\end{array}$

URL: http://Www octoraro.com/

\section{Landenberg}

North Creek Nurseries, Inc.

338 N. Creek Rd.

Landenberg. PA

19350

PHONE: (610) 255-0100

$\begin{array}{ll}\square \text { TREE } & \square \text { LOCAL TREE } \\ \square \text { SHRUB } & \square \text { LOCAL SHRUB } \\ \nabla \text { GRASS } & \square \text { LOCAL GRASS } \\ \square \text { FORBS } & \square \text { LOCAL FORBS } \\ \square \text { WETLAND } & \square \text { LOCAL WETLAND }\end{array}$

URL: www.nothcreeknurseries.com 
Lewisburg

Maple Hill Farms

PO Box 648

Lewisburg. PA

17837

PHONE: (570) 524-0791

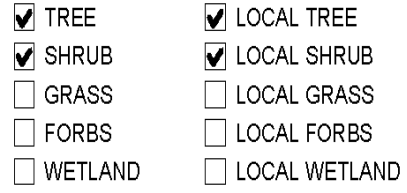

URL: none currently available

Mahaffey

Hanchars Superior Trees

RD1, Box 118"

$\nabla$ TREE $\quad \nabla$ LOCAL TREE

Mahaffey, PA

$\nabla$ SHRUB $\nabla$ LOCAL SHRUB

15757

$\square$ GRASS $\square$ LOCAL GRASS

PHONE: (814) 277-6674

$\square$ FORBS $\quad \square$ LOCAL FORBS

$\nabla$ WETLAND $\quad \nabla$ LOCAL WETLAND

URL: none currently available

Malvern

Fisher \& Son Co. Inc.

237 E. King St.

Malvern, PA

19355

PHONE: (800) 262-2127

$\begin{array}{ll}\square \text { TREE } & \square \text { LOCAL TREE } \\ \square \text { SHRUB } & \square \text { LOCAL SHRUB } \\ \nabla \text { GRASS } & \square \text { LOCAL GRASS } \\ \square \text { FORBS } & \square \text { LOCAL FORBS } \\ \square \text { WETLAND } & \square \text { LOCAL WETLAND }\end{array}$

URL: www.fisherandson.com

Maple Glen

Garys Perennials

1122 E. Welsh Rd

Maple Glen, PA

19002

PHONE: (800) 898-6653

$\begin{array}{ll}\square \text { TREE } & \square \text { LOCAL TREE } \\ \square \text { SHRUB } & \square \text { LOCAL SHRUB } \\ \square \text { GRASS } & \square \text { LOCAL GRASS } \\ \square \text { FORBS } & \square \text { LOCAL FORBS } \\ \square \text { WETLAND } & \square \text { LOCAL WETLAND }\end{array}$

URL: none currently available

Meadville

Ernst Conservation Seeds

9006 Mercer Pike

Meadville, PA

16335

PHONE: (800) 873-3321

$\begin{array}{ll}\square \text { TREE } & \square \text { LOCAL TREE } \\ \nabla \text { SHRUB } & \square \text { LOCAL SHRUB } \\ \nabla \text { GRASS } & \square \text { LOCAL GRASS } \\ \nabla \text { FORBS } & \square \text { LOCAL FORBS } \\ \square \text { WETLAND } & \square \text { LOCAL WETLAND }\end{array}$

URL: www.ernstseed.com

New Hope

Bowmans Hill Wildflower Preserve

P.O.Box 685

$\nabla$ TREE $\quad \square$ LOCAL TREE

New Hope, PA

18938

$\checkmark$ SHRUB

$\checkmark$ GRASS

$\square$ LOCAL SHRUB

PHONE: (215) 862-2924

$\square$ FORBS

$\square$ LOCAL GRASS

URL: http://Www bhwp.orgl 
Penfield

Johnstons Nursery

Road 1, Box $189 \mathrm{C}$

Penfield, PA

15849

PHONE: (814) 765-9081

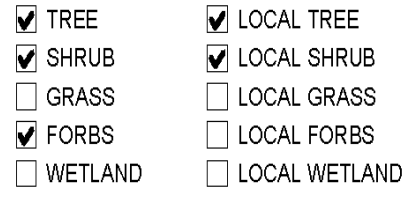

URL: www.johnstonsnursery.com

\section{Penn Run}

Pikes Peak Nurseries

8289 Route 422 Hwy E

Penn Run, PA

15765

PHONE: (412) 463-7747

$\begin{array}{ll}\nabla \text { TREE } & \nabla \text { LOCAL TREE } \\ \nabla \text { SHRUB } & \square \text { LOCAL SHRUB } \\ \square \text { GRASS } & \square \text { LOCAL GRASS } \\ \square \text { FORBS } & \square \text { LOCAL FORBS } \\ \square \text { WETLAND } & \square \text { LOCAL WETLAND }\end{array}$

URL: www.pikespeaknurseries.net/

\section{Petersburg}

Better Forest Tree Seeds

$\begin{array}{lll}\text { RD 1. Box 636 } & \square \text { TREE } & \square \text { LOCAL TREE } \\ \text { Petersburg, PA } & \square \text { SHRUB } & \square \text { LOCAL SHRUB } \\ \text { 16669 } & \square \text { GRASS } & \square \text { LOCAL GRASS } \\ \text { PHONE: (814) 667-3666 } & \square \text { FORBS } & \square \text { LOCAL FORBS } \\ & \square \text { WETLAND } & \square \text { LOCAL WETLAND }\end{array}$

URL: none currently available

Pipersville

Aquascapes Unlimited

PO Box 364

$\checkmark$ TREE $\quad \nabla$ LOCAL TREE

Pipersville, PA

18947

$\checkmark$ SHRUB

$\nabla$ LOCAL SHRUB

PHONE: (215) 766-8151

$\checkmark$ GRASS $\quad \nabla$ LOCAL GRASS

$\nabla$ FORBS $\quad \nabla$ LOCAL FORBS

$\checkmark$ WETLAND $\square$ LOCAL WETLAND

URL: none currently available

Pittsburgh

Sylvania Natives

5502 Kamin St.

Pittsburgh, PA

15217

PHONE: (412) 421-8551

$\begin{array}{ll}\square \text { TREE } & \square \text { LOCAL TREE } \\ \square \text { SHRUB } & \square \text { LOCAL SHRUB } \\ \square \text { GRASS } & \square \text { LOCAL GRASS } \\ \nabla \text { FORBS } & \square \text { LOCAL FORBS } \\ \square \text { WETLAND } & \square \text { LOCAL WETLAND }\end{array}$

URL: WwW.Sylvanianatives.com

\section{Port Matilda}

Limerock Ormanental Grasses

70 Saw Mill Rd.

$\square$ TREE $\quad \square$ LOCAL TREE

Port Matilda. PA

16870

$\square$ SHRUB

$\square$ LOCAL SHRUB

PHONE: (814) 692-2272

$\checkmark$ GRASS

$\square$ LOCAL GRASS

$\square$ FORBS

$\square$ LOCAL FORBS

$\square$ WETLAND $\square$ LOCAL WETLAND

URL: www.limerockgrasses.com 
Prospect

Eisler Nurseries

1031 Newcastle Rd

Prospect, PA

16052

PHONE: (412) 865-2830

$\begin{array}{ll}\nabla \text { TREE } & \square \text { LOCAL TREE } \\ \square \text { SHRUB } & \square \text { LOCAL SHRUB } \\ \square \text { GRASS } & \square \text { LOCAL GRASS } \\ \square \text { FORBS } & \square \text { LOCAL FORBS } \\ \square \text { WETLAND } & \square \text { LOCAL WETLAND }\end{array}$

URL: www.eislemurseries.com

\section{Sagamore}

Flickingers Nursery

Box 245

$\nabla$ TREE $\quad \nabla$ LOCAL TREE

Sagamore, PA

16250

$\checkmark$ SHRUB

$\checkmark$ LOCAL SHRUB

PHONE: (412) 783-6528

$\square$ GRASS $\square$ LOCAL GRASS

$\square$ FORBS $\square$ LOCAL FORBS

$\square$ WETLAND $\square$ LOCAL WETLAND

URL: none currently available

\section{Schwenksville}

Behmerwald Nursery

4904 Garges Rd

Schwenksville, PA

19473

PHONE: (610) 287-0480

$\begin{array}{ll}\nabla \text { TREE } & \square \text { LOCAL TREE } \\ \square \text { SHRUB } & \square \text { LOCAL SHRUB } \\ \square \text { GRASS } & \square \text { LOCAL GRASS } \\ \square \text { FORBS } & \square \text { LOCAL FORBS } \\ \square \text { WETLAND } & \square \text { LOCAL WETLAND }\end{array}$

URL: none currently available

\section{Scranton}

Ecoscientific Solutions LLC

930 Meadow Ave Suite B

Scranton, PA

18505

PHONE: (570) 496-1000

$\begin{array}{ll}\square \text { TREE } & \square \text { LOCAL TREE } \\ \square \text { SHRUB } & \square \text { LOCAL SHRUB } \\ \square \text { GRASS } & \square \text { LOCAL GRASS } \\ \square \text { FORBS } & \square \text { LOCAL FORBS } \\ \square \text { WETLAND } & \square \text { LOCAL WETLAND }\end{array}$

URL: www.ecoscientificsolutions.com

\section{Warminster}

Burpees Seeds

300 Park Ave.

Warminster, PA

18974

PHONE: (800) 333-5808

$\begin{array}{ll}\square \text { TREE } & \square \text { LOCAL TREE } \\ \square \text { SHRUB } & \square \text { LOCAL SHRUB } \\ \square \text { GRASS } & \square \text { LOCAL GRASS } \\ \square \text { FORBS } & \square \text { LOCAL FORBS } \\ \square \text { WETLAND } & \square \text { LOCAL WETLAND }\end{array}$

URL: www.burpees.com

\section{Waynesboro}

Appalachian Nurseries

Box 87

$\nabla$ TREE $\quad \nabla$ LOCAL TREE

Waynesboro, PA

17268

$\checkmark$ SHRUB

$\checkmark$ LOCAL SHRUB

PHONE: (717) 762-4733

$\square$ GRASS $\square$ LOCAL GRASS

$\square$ FORBS $\quad \square$ LOCAL FORBS

$\square$ WETLAND $\square$ LOCAL WETLAND

URL: www.appnursery.com 
West Grove

Natural Landscapes

354 N. Jennersville Rd.

West Grove, PA

19390

PHONE: (610) 869-3788

URL: none currently available

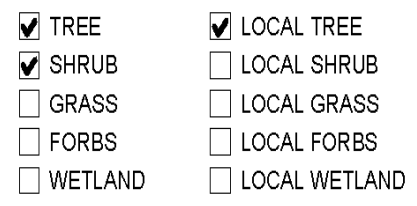

$\square$ WETLAND

$\square$ LOCAL WETLAND

\section{Worcester}

David Brothers - Bean Read Nursery
PO Box 123

Worcester, PA

19403

PHONE: (610) 584-1550

$\begin{array}{ll}\nabla \text { TREE } & \nabla \text { LOCAL TREE } \\ \nabla \text { SHRUB } & \nabla \text { LOCAL SHRUB } \\ \square \text { GRASS } & \square \text { LOCAL GRASS } \\ \square \text { FORBS } & \square \text { LOCAL FORBS } \\ \nabla \text { WETLAND } & \nabla \text { LOCAL WETLAND }\end{array}$

URL: none currently available

\section{Rhode Island}

Exeter

Bald Hill Nurseries, Inc.

90 Ten Rod Rd.

Exeter, RI

02822

PHONE: (401) 294-9100

$\begin{array}{ll}\nabla \text { TREE } & \nabla \text { LOCAL TREE } \\ \nabla \text { SHRUB } & \boldsymbol{\nabla} \text { LOCAL SHRUB } \\ \square \text { GRASS } & \square \text { LOCAL GRASS } \\ \square \text { FORBS } & \square \text { LOCAL FORBS } \\ \square \text { WETLAND } & \boldsymbol{\nabla} \text { LOCAL WETLAND }\end{array}$

URL: www.baldhillnurseries.com

\section{South Carolina}

Aiken

Weyerhauser $\mathrm{Co}$

169 Weyerhouser Rd.

Aiken, SC

29801

PHONE: (803) 649-0489

$\begin{array}{ll}\square \text { TREE } & \square \text { LOCAL TREE } \\ \square \text { SHRUB } & \square \text { LOCAL SHRUB } \\ \square \text { GRASS } & \square \text { LOCAL GRASS } \\ \square \text { FORBS } & \square \text { LOCAL FORBS } \\ \square \text { WETLAND } & \square \text { LOCAL WETLAND }\end{array}$

URL: www.weyerhaeuser.com

Woodlanders, Inc.

$\begin{array}{lll}1128 \text { Colleton Ave. } & \nabla \text { TREE } & \square \text { LOCAL TREE } \\ \text { Aiken, SC } & \nabla \text { SHRUB } & \square \text { LOCAL SHRUB } \\ 29801 & \nabla \text { GRASS } & \square \text { LOCAL GRASS } \\ \text { PHONE: (803) 648-7522 } & \nabla \text { FORBS } & \square \text { LOCAL FORBS } \\ & \nabla \text { WETLAND } & \square \text { LOCAL WETLAND }\end{array}$

URL: www.woodlanders.net/

\section{Columbia}

South Carolina Forestry Commission

5500 Broad River Rd

Columbia, SC

29212

PHONE: (803) 896-8800

$\begin{array}{ll}\nabla \text { TREE } & \square \text { LOCAL TREE } \\ \square \text { SHRUB } & \square \text { LOCAL SHRUB } \\ \square \text { GRASS } & \square \text { LOCAL GRASS } \\ \square \text { FORBS } & \square \text { LOCAL FORBS } \\ \square \text { WETLAND } & \square \text { LOCAL WETLAND }\end{array}$

URL: http:/Wwww.state.sc.us/forest/ 


\section{Eastover}

Wavering Place Gardens \& Nursery

$\begin{array}{lll}\text { Rt. } 2 \text { Box } 269 & \square \text { TREE } & \square \text { LOCAL TREE } \\ \text { Eastover, SC } & \square \text { SHRUB } & \square \text { LOCAL SHRUB } \\ 29044 & \square \text { GRASS } & \square \text { LOCAL GRASS } \\ \text { PHONE: (803) 783-1682 } & \square \text { FORBS } & \square \text { LOCAL FORBS } \\ & \square \text { WETLAND } & \square \text { LOCAL WETLAND }\end{array}$

URL: none currently available

\section{Greenwood}

Park Seed Wholesale

Cokesbury Rd. Hwy 254 North

Greenwood, SC

29647

PHONE: (800) 845-3366

$\begin{array}{ll}\square \text { TREE } & \square \text { LOCAL TREE } \\ \square \text { SHRUB } & \square \text { LOCAL SHRUB } \\ \square \text { GRASS } & \square \text { LOCAL GRASS } \\ \square \text { FORBS } & \square \text { LOCAL FORBS } \\ \square \text { WETLAND } & \square \text { LOCAL WETLAND }\end{array}$

URL: www.parkwholesale.com

\section{Hodges}

Wayside Gardens

$\begin{array}{lll}\text { 1 Garden lane } & \nabla \text { TREE } & \nabla \text { LOCAL TREE } \\ \text { Hodges, SC } & \nabla \text { SHRUB } & \nabla \text { LOCAL SHRUB } \\ 29695 & \nabla \text { GRASS } & \square \text { LOCAL GRASS } \\ \text { PHONE: (800) 845-1124 } & \square \text { FORBS } & \nabla \text { LOCAL FORBS } \\ & \square \text { WETLAND } & \square \text { LOCAL WETLAND }\end{array}$

URL: www.waysidegardens.com

\section{John's Island}

Charleston Aquatic Nurseries

3095 Canal Bridge Rd.

John's Island, SC

29455

PHONE: (803) 559-3151

$\begin{array}{ll}\square \text { TREE } & \square \text { LOCAL TREE } \\ \square \text { SHRUB } & \square \text { LOCAL SHRUB } \\ \square \text { GRASS } & \square \text { LOCAL GRASS } \\ \square \text { FORBS } & \square \text { LOCAL FORBS } \\ \square \text { WETLAND } & \square \text { LOCAL WETLAND }\end{array}$

URL: www.charlestonaquatic.com

$\begin{array}{lll}\text { Moncks Corner } & & \\ \text { Carolina Nurseries } & \\ 739 \text { Gaillard Rd. } & \square \text { TREE } & \square \text { LOCAL TREE } \\ \text { Moncks Corner, SC } & \square \text { SHRUB } & \square \text { LOCAL SHRUB } \\ 29461 & \square \text { GRASS } & \square \text { LOCAL GRASS } \\ \text { PHONE: (800) 845-2065 } & \square \text { FORBS } & \square \text { LOCAL FORBS } \\ & \square \text { WETLAND } & \square \text { LOCAL WETLAND }\end{array}$

URL: www.carolinanurseries.com

\section{Myrtle Beach}

Coastal Gardens

4611 Socastee Blvd. "

Myrtle Beach, SC

29588

PHONE: (843) 293-2000

$\begin{array}{ll}\nabla \text { TREE } & \square \text { LOCAL TREE } \\ \nabla \text { SHRUB } & \square \text { LOCAL SHRUB } \\ \nabla \text { GRASS } & \square \text { LOCAL GRASS } \\ \square \text { FORBS } & \square \text { LOCAL FORBS } \\ \square \text { WETLAND } & \square \text { LOCAL WETLAND }\end{array}$

URL: none currently available 


\section{South Dakota}

Aberdeen

Eugene Raap

305 N. Boyd St.

Aberdeen, SD

57401

PHONE: (605) 325-3308

$\begin{array}{ll}\square \text { TREE } & \square \text { LOCAL TREE } \\ \square \text { SHRUB } & \square \text { LOCAL SHRUB } \\ \square \text { GRASS } & \square \text { LOCAL GRASS } \\ \square \text { FORBS } & \square \text { LOCAL FORBS } \\ \square \text { WETLAND } & \square \text { LOCAL WETLAND }\end{array}$

URL: none currently available

\section{Batesland}

Leo Kratovil

P.O. Box 55

Batesland, SD

57716

$\square$ TREE

$\square$ SHRUB

$\checkmark$ GRASS

$\square$ LOCAL TREE

$\square$ FORBS

$\square$ LOCAL SHRUB

PHONE: (605) 288-1933

$\square$ WETLAND

$\checkmark$ LOCAL GRASS

$\square$ LOCAL FORBS

$\square$ LOCAL WETLAND

URL: none currently available

\section{Belle Fourche}

Dakota Mill and Grain

P.O. Box 608

Belle Fourche, SD

57717

PHONE: (605) 892-2543

$\begin{array}{ll}\square \text { TREE } & \square \text { LOCAL TREE } \\ \square \text { SHRUB } & \square \text { LOCAL SHRUB } \\ \square \text { GRASS } & \square \text { LOCAL GRASS } \\ \square \text { FORBS } & \square \text { LOCAL FORBS } \\ \square \text { WETLAND } & \square \text { LOCAL WETLAND }\end{array}$

URL: www.dakotamill.com

\section{Big Stone City}

Roger McCulloch

48025 145th St.

Big Stone City, SD

57216

PHONE: (605) 432-4129

$\begin{array}{ll}\square \text { TREE } & \square \text { LOCAL TREE } \\ \square \text { SHRUB } & \square \text { LOCAL SHRUB } \\ \square \text { GRASS } & \square \text { LOCAL GRASS } \\ \square \text { FORBS } & \square \text { LOCAL FORBS } \\ \square \text { WETLAND } & \square \text { LOCAL WETLAND }\end{array}$

URL: none currently available

\section{Bridgewater}

Coyote Seed Mills Inc.

Box 16 Highway 262

Bridgewater, SD

57319

PHONE: (605) 729-2511

$\begin{array}{ll}\square \text { TREE } & \square \text { LOCAL TREE } \\ \square \text { SHRUB } & \square \text { LOCAL SHRUB } \\ \square \text { GRASS } & \square \text { LOCAL GRASS } \\ \square \text { FORBS } & \square \text { LOCAL FORBS } \\ \square \text { WETLAND } & \square \text { LOCAL WETLAND }\end{array}$

URL: none currently available

\section{Bristol}

Hansmeier \& Son

Box 136

Bristol, SD

57219

PHONE: (605) 492-3611

$\begin{array}{ll}\square \text { TREE } & \square \text { LOCAL TREE } \\ \square \text { SHRUB } & \square \text { LOCAL SHRUB } \\ \square \text { GRASS } & \square \text { LOCAL GRASS } \\ \square \text { FORBS } & \square \text { LOCAL FORBS } \\ \square \text { WETLAND } & \square \text { LOCAL WETLAND }\end{array}$

URL: none currently available 


\section{Brookings}

Milborne Seeds, Inc.

3127 Hwy 14 Bypass

Brookings, SD

57006

PHONE: (605) 697-6305

URL: www.milbornseeds.com

Millborn Seeds, Inc.

3127 Hwy. 14 BYP

Brookings, SD

57006

PHONE: (605) 697-7464

URL: none currently available

Sexauer Co.

100 Main Ave

Brookings, SD

57006

PHONE: (605) 696-7924

URL: none currently available

Cavour

Pearl View Seeds

21697408 th Ave.

Cavour, SD

57324

PHONE: (605) 352-5933

URL: www.pearlviewseeds.com

$\begin{array}{ll}\square \text { TREE } & \square \text { LOCAL TREE } \\ \square \text { SHRUB } & \square \text { LOCAL SHRUB } \\ \square \text { GRASS } & \square \text { LOCAL GRASS } \\ \nabla \text { FORBS } & \square \text { LOCAL FORBS } \\ \square \text { WETLAND } & \square \text { LOCAL WETLAND }\end{array}$

$\begin{array}{ll}\square \text { TREE } & \square \text { LOCAL TREE } \\ \square \text { SHRUB } & \square \text { LOCAL SHRUB } \\ \square \text { GRASS } & \square \text { LOCAL GRASS } \\ \square \text { FORBS } & \square \text { LOCAL FORBS } \\ \square \text { WETLAND } & \square \text { LOCAL WETLAND }\end{array}$

$\begin{array}{ll}\square \text { TREE } & \square \text { LOCAL TREE } \\ \square \text { SHRUB } & \square \text { LOCAL SHRUB } \\ \nabla \text { GRASS } & \square \text { LOCAL GRASS } \\ \nabla \text { FORBS } & \square \text { LOCAL FORBS } \\ \square \text { WETLAND } & \square \text { LOCAL WETLAND }\end{array}$

Colome

Bee Seeds

P.O. Box 103

Colome, SD

57528

PHONE: (605) 842-3232

$\begin{array}{ll}\nabla \text { TREE } & \nabla \text { LOCAL TREE } \\ \nabla \text { SHRUB } & \nabla \text { LOCAL SHRUB } \\ \nabla \text { GRASS } & \nabla \text { LOCAL GRASS } \\ \nabla \text { FORBS } & \nabla \text { LOCAL FORBS } \\ \nabla \text { WETLAND } & \checkmark \text { LOCAL WETLAND }\end{array}$

$\checkmark$ WETLAND $\nabla$ LOCAL WETLAND

$\begin{array}{ll}\square \text { TREE } & \square \text { LOCAL TREE } \\ \square \text { SHRUB } & \square \text { LOCAL SHRUB } \\ \nabla \text { GRASS } & \nabla \text { LOCAL GRASS } \\ \square \text { FORBS } & \nabla \text { LOCAL FORBS } \\ \square \text { WETLAND } & \square \text { LOCAL WETLAND }\end{array}$

URL: none currently available

Faith

Northern Plains Seed

N. Main

Faith, SD

57626

PHONE: (605) 967-2102

$\begin{array}{ll}\square \text { TREE } & \square \text { LOCAL TREE } \\ \square \text { SHRUB } & \square \text { LOCAL SHRUB } \\ \square \text { GRASS } & \square \text { LOCAL GRASS } \\ \square \text { FORBS } & \square \text { LOCAL FORBS } \\ \square \text { WETLAND } & \square \text { LOCAL WETLAND }\end{array}$

URL: none currently available 
Midland

Goldenwillow Seeds, Inc.

HCR 69, Box 553"

Midland, SD

57552

PHONE: (605) 843-2187

$\begin{array}{ll}\square \text { TREE } & \square \text { LOCAL TREE } \\ \square \text { SHRUB } & \square \text { LOCAL SHRUB } \\ \square \text { GRASS } & \square \text { LOCAL GRASS } \\ \square \text { FORBS } & \square \text { LOCAL FORBS } \\ \square \text { WETLAND } & \square \text { LOCAL WETLAND }\end{array}$

URL: none currently available

Miller

Bertsch Ranch

HC 76, Box 137"

Miller, SD

57362

L LOCAL TREE

PHONE: (605) 853-2341

$\square$ LOCAL SHRUB

$\checkmark$ GRASS

$\checkmark$ LOCAL GRASS

$\begin{array}{ll}\square \text { FORBS } & \square \text { LOCAL FORBS } \\ \square \text { WETLAND } & \square \text { LOCAL WETLAND }\end{array}$

URL: none currently available

Wilbers Seed Solutions

800 N. Broadway, Box 41"

Miller, SD

57362

PHONE: (605) 853-2414

$\begin{array}{ll}\square \text { TREE } & \square \text { LOCAL TREE } \\ \square \text { SHRUB } & \square \text { LOCAL SHRUB } \\ \square \text { GRASS } & \square \text { LOCAL GRASS } \\ \square \text { FORBS } & \square \text { LOCAL FORBS } \\ \square \text { WETLAND } & \square \text { LOCAL WETLAND }\end{array}$

URL: wWw.seedsolutions.com

New Underwood

Scott Phillips

Box 17

New Underwood, SD

57761

PHONE: (605) 985-5573

$\begin{array}{ll}\square \text { TREE } & \square \text { LOCAL TREE } \\ \square \text { SHRUB } & \square \text { LOCAL SHRUB } \\ \square \text { GRASS } & \square \text { LOCAL GRASS } \\ \square \text { FORBS } & \square \text { LOCAL FORBS } \\ \square \text { WETLAND } & \square \text { LOCAL WETLAND }\end{array}$

URL: none currently available

Platte

Den Besten Seed Co.

West Highway 44

Platte, SD

57369

PHONE: (605) 337-3318

$\begin{array}{ll}\square \text { TREE } & \square \text { LOCAL TREE } \\ \square \text { SHRUB } & \square \text { LOCAL SHRUB } \\ \square \text { GRASS } & \square \text { LOCAL GRASS } \\ \square \text { FORBS } & \square \text { LOCAL FORBS } \\ \square \text { WETLAND } & \square \text { LOCAL WETLAND }\end{array}$

URL: www.denbestenseed.com

\section{Rapid City}

Rapid Feed \& Seed

3640 S. Hwy 79 Box 679

Rapid City SD

57709

PHONE: (605) 348-0059

$\begin{array}{ll}\square \text { TREE } & \square \text { LOCAL TREE } \\ \square \text { SHRUB } & \square \text { LOCAL SHRUB } \\ \square \text { GRASS } & \square \text { LOCAL GRASS } \\ \square \text { FORBS } & \square \text { LOCAL FORBS } \\ \square \text { WETLAND } & \square \text { LOCAL WETLAND }\end{array}$

URL: none currently available 
Warren Chemical \& Equipment

2680 Commerce Rd

$\begin{array}{ll}\square \text { TREE } & \square \text { LOCAL TREE } \\ \square \text { SHRUB } & \square \text { LOCAL SHRUB } \\ \square \text { GRASS } & \square \text { LOCAL GRASS } \\ \square \text { FORBS } & \square \text { LOCAL FORBS } \\ \square \text { WETLAND } & \square \text { LOCAL WETLAND }\end{array}$

Rapid City, SD

57702

PHONE: (800) 658-5457

$\square$ WETLAND $\square$ LOCAL WETLAND

URL: none currently available

\section{Sioux Falls}

Northern Plains Seed $C_{0}$.

$1320 \mathrm{~N}$. Ellis Rd.

Sioux Falls, SD

57101

PHONE: (605) 336-0623

$\begin{array}{ll}\square \text { TREE } & \square \text { LOCAL TREE } \\ \square \text { SHRUB } & \square \text { LOCAL SHRUB } \\ \nabla \text { GRASS } & \square \text { LOCAL GRASS } \\ \square \text { FORBS } & \square \text { LOCAL FORBS } \\ \square \text { WETLAND } & \square \text { LOCAL WETLAND }\end{array}$

URL: none currently available

\section{Sturgis}

Ed Thompson

HC 75. Box 134"

Sturgis, SD

57785

PHONE: (605) 347-3183

$\begin{array}{ll}\nabla \text { TREE } & \square \text { LOCAL TREE } \\ \square \text { SHRUB } & \square \text { LOCAL SHRUB } \\ \nabla \text { GRASS } & \square \text { LOCAL GRASS } \\ \nabla \text { FORBS } & \square \text { LOCAL FORBS } \\ \nabla \text { WETLAND } & \square \text { LOCAL WETLAND }\end{array}$

URL: none currently available

Foothills Seed

2323 S. Junction Ave

Sturgis, SD

57785

PHONE: (605) 347-4578

$\begin{array}{ll}\square \text { TREE } & \square \text { LOCAL TREE } \\ \square \text { SHRUB } & \square \text { LOCAL SHRUB } \\ \square \text { GRASS } & \square \text { LOCAL GRASS } \\ \square \text { FORBS } & \square \text { LOCAL FORBS } \\ \square \text { WETLAND } & \square \text { LOCAL WETLAND }\end{array}$

URL: none currently available

\section{Union Center}

Cammack Ranch Supply, Inc.

PO Box 2

Union Center, SD

57787

PHONE: (605) 985-5591

$\begin{array}{ll}\square \text { TREE } & \square \text { LOCAL TREE } \\ \square \text { SHRUB } & \square \text { LOCAL SHRUB } \\ \square \text { GRASS } & \square \text { LOCAL GRASS } \\ \square \text { FORBS } & \square \text { LOCAL FORBS } \\ \square \text { WETLAND } & \square \text { LOCAL WETLAND }\end{array}$

URL: www.cammackranchsupply.com

\section{Watertown}

Discount Seeds

P.O. Box 84

Watertown, SD

57201

PHONE: (605) 886-5888

$\begin{array}{ll}\square \text { TREE } & \square \text { LOCAL TREE } \\ \square \text { SHRUB } & \square \text { LOCAL SHRUB } \\ \square \text { GRASS } & \square \text { LOCAL GRASS } \\ \square \text { FORBS } & \square \text { LOCAL FORBS } \\ \square \text { WETLAND } & \square \text { LOCAL WETLAND }\end{array}$

URL: none currently available 
Tennessee

Adams

Felts Seed \& Supply

6052 Highway $41 \mathrm{~N}$

Adams, TN

37010

PHONE: (615) 696-2431

$\begin{array}{ll}\square \text { TREE } & \square \text { LOCAL TREE } \\ \square \text { SHRUB } & \square \text { LOCAL SHRUB } \\ \square \text { GRASS } & \square \text { LOCAL GRASS } \\ \square \text { FORBS } & \square \text { LOCAL FORBS } \\ \square \text { WETLAND } & \square \text { LOCAL WETLAND }\end{array}$

URL: none currently available

\section{Andersonville}

Sunlight Gardens

174 Golden La.

Andersonville, TN

37705

$\square$ TREE

$\checkmark$ SHRUB

$\square$ LOCAL TREE

$\checkmark$ GRASS

$\square$ LOCAL SHRUB

PHONE: (800) 272-7396

$\checkmark$ FORBS

$\square$ LOCAL GRASS

$\square$ WETLAND

$\square$ LOCAL FORBS

$\square$ LOCAL WETLAND

URL: www.sunlightgardens.com

Arlington

Dello Nurseries, Inc.

11034 Hwy. 64

Arlington, TN

38002

PHONE: (901) 867-3511

$\begin{array}{ll}\nabla \text { TREE } & \square \text { LOCAL TREE } \\ \nabla \text { SHRUB } & \square \text { LOCAL SHRUB } \\ \nabla \text { GRASS } & \square \text { LOCAL GRASS } \\ \nabla \text { FORBS } & \square \text { LOCAL FORBS } \\ \square \text { WETLAND } & \square \text { LOCAL WETLAND }\end{array}$

URL: none currently available

\section{Beersheba Springs}

Big Springs Nursery \& Wildflowers

PO Box 513

Beersheba Springs, TN

37305

PHONE: (913) 692-3604

$\checkmark$ TREE

$\checkmark$ SHRUB

$\square$ GRASS

$\checkmark$ FORBS

$\square$ WETLAND $\square$ LOCAL TREE

$\square$ LOCAL SHRUB

$\square$ LOCAL GRASS

$\square$ LOCAL FORBS

$\square$ LOCAL WETLAND

URL: none currently available

Delano

Tennessee Division of Forestry / East Tennessee Nursery

PO Box 59

Delano, TN

37325

PHONE: (423) 263-1626

$\checkmark$ TREE

$\square$ SHRUB

$\square$ GRASS

$\square$ FORBS

$\checkmark$ WETLAND $\checkmark$ LOCAL TREE

$\square$ LOCAL SHRUB

$\square$ LOCAL GRASS

$\square$ LOCAL FORBS

$\checkmark$ LOCAL WETLAND

URL: http:/Www state.tn. us/agriculturefforestry/

\section{Greenback}

Native Gardens

5737 Fisher Lane

Greenback, TN

37742

PHONE: (865) 856-0220

$\boldsymbol{\nabla}$ TREE

$\checkmark$ SHRUB

$\nabla$ GRASS

$\checkmark$ FORBS

$\checkmark$ WETLAND $\square$ LOCAL TREE

$\square$ LOCAL SHRUB

$\square$ LOCAL GRASS

$\square$ LOCAL FORBS

$\square$ LOCAL WETLAND

URL: http:/Nww'native-gardens.com/ 


\begin{tabular}{|c|c|c|}
\hline \multicolumn{3}{|l|}{ McMinnville } \\
\hline \multicolumn{3}{|l|}{ Boyd Bros. Nursery Co. } \\
\hline Box 788 & $\nabla$ TREE & $\nabla$ LOCAL TREE \\
\hline McMinnville, TN & $\checkmark$ SHRUB & $\nabla$ LOCAL SHRUB \\
\hline 37110 & $\square$ GRASS & $\square$ LOCAL GRASS \\
\hline \multirow[t]{2}{*}{ PHONE: (931) 668-9898 } & $\nabla$ FORBS & $\square$ LOCAL FORBS \\
\hline & $\square$ WETLAND & $\square$ LOCAL WETLAND \\
\hline \multicolumn{3}{|c|}{ URL: none currently available } \\
\hline \multicolumn{3}{|l|}{ Forest Nursery $\mathrm{C}_{0} .$, Inc. } \\
\hline 2362 Beersheba Hwy & $\checkmark$ TREE & $\nabla$ LOCAL TREE \\
\hline McMinnville, TN & SHRUB & $\nabla$ LOCAL SHRUB \\
\hline 37110 & $\square$ GRASS & $\square$ LOCAL GRASS \\
\hline \multirow[t]{2}{*}{ PHONE: (931) 473-2133 } & $\nabla$ FORBS & $\nabla$ LOCAL FORBS \\
\hline & $\square$ WETLAND & $\square$ LOCAL WETLAND \\
\hline \multicolumn{3}{|c|}{ URL: http://Www thnursery.com/Forest/ } \\
\hline \multicolumn{3}{|l|}{ Greenwood Nursery } \\
\hline 636 Myers Grove Rd. & $\nabla$ TREE & $\checkmark$ LOCAL TREE \\
\hline McMinnville, TN & $\nabla$ SHRUB & $\nabla$ LOCAL SHRUB \\
\hline 37110 & $\square$ GRASS & $\square$ LOCAL GRASS \\
\hline \multirow{2}{*}{ PHONE: $(800)$ 426-0958 } & $\nabla$ FORBS & $\square$ LOCAL FORBS \\
\hline & $\square$ WETLAND & $\square$ LOCAL WETLAND \\
\hline \multicolumn{3}{|c|}{ URL: http:/WWww greenwoodnursery.com/ } \\
\hline \multicolumn{3}{|c|}{ Hillis Nursery Co., Inc. } \\
\hline 92 Gardner Rd. & $\nabla$ TREE & $\nabla$ LOCAL TREE \\
\hline McMinnville, TN & $\checkmark$ SHRUB & $\nabla$ LOCAL SHRUB \\
\hline 37110 & $\square$ GRASS & $\square$ LOCAL GRASS \\
\hline \multirow[t]{2}{*}{ PHONE: (931) 668-4364 } & $\square$ FORBS & $\square$ LOCAL FORBS \\
\hline & $\nabla$ WETLAND & $\square$ LOCAL WETLAND \\
\hline \multicolumn{3}{|c|}{ URL: none currently available } \\
\hline \multicolumn{3}{|l|}{ Myers Cove Nursery, Inc. } \\
\hline Box 191 & $\boldsymbol{v}$ TREE & $\nabla$ LOCAL TREE \\
\hline McMinnville, TN & $\checkmark$ SHRUB & $\nabla$ LOCAL SHRUB \\
\hline 37110 & $\square$ GRASS & $\square$ LOCAL GRASS \\
\hline \multirow[t]{2}{*}{ PHONE: (615) 668-3155 } & $\checkmark$ FORBS & $\square$ LOCAL FORBS \\
\hline & $\square$ WETLAND & $\square$ LOCAL WETLAND \\
\hline \multicolumn{3}{|c|}{ URL: http:/Wwww.tnnursery.com/MyersCovel } \\
\hline \multicolumn{3}{|l|}{ Tennessee Bush Farms } \\
\hline 11343 Beersheeba Hwy & $\checkmark$ TREE & $\nabla$ LOCAL TREE \\
\hline McMinnville, TN & $\square$ SHRUB & $\nabla$ LOCAL SHRUB \\
\hline 37110 & $\square$ GRASS & $\square$ LOCAL GRASS \\
\hline \multirow[t]{2}{*}{ PHONE: (931) 692-3624 } & $\square$ FORBS & $\square$ LOCAL FORBS \\
\hline & $\square$ WETLAND & $\square$ LOCAL WETLAND \\
\hline \multicolumn{3}{|c|}{ URL: none currently available } \\
\hline \multicolumn{3}{|l|}{ Triangle Nursery, Inc. } \\
\hline 8526 Beersheba Hwy & $\nabla$ TREE & $\nabla$ LOCAL TREE \\
\hline McMinnville, TN & $\square$ SHRUB & $\checkmark$ LOCAL SHRUB \\
\hline 37110 & $\square$ GRASS & $\square$ LOCAL GRASS \\
\hline \multirow[t]{2}{*}{ PHONE: (931) 668-8022 } & $\square$ FORBS & $\square$ LOCAL FORBS \\
\hline & $\square$ WETLAND & $\square$ LOCAL WETLAND \\
\hline
\end{tabular}

URL: www.trianglenursery.com 
Wanamaker Nursery, Inc.

12695 Beersheba Hwy

McMinnville, TN

37110

PHONE: (931) 692-3763

$\begin{array}{ll}\nabla \text { TREE } & \square \text { LOCAL TREE } \\ \nabla \text { SHRUB } & \square \text { LOCAL SHRUB } \\ \square \text { GRASS } & \square \text { LOCAL GRASS } \\ \square \text { FORBS } & \square \text { LOCAL FORBS } \\ \square \text { WETLAND } & \square \text { LOCAL WETLAND }\end{array}$

URL: none currently available

Warren County Nursery

6492 Beersheba Hwy

McMinnville, TN

37110

PHONE: (931) 668-8941

$\begin{array}{ll}\nabla \text { TREE } & \nabla \text { LOCAL TREE } \\ \nabla \text { SHRUB } & \nabla \text { LOCAL SHRUB } \\ \nabla \text { GRASS } & \nabla \text { LOCAL GRASS } \\ \nabla \text { FORBS } & \nabla \text { LOCAL FORBS } \\ \nabla \text { WETLAND } & \nabla \text { LOCAL WETLAND }\end{array}$

URL: http:/Www tnnursery.comMCN/

Memphis

Seeds, Inc.

2435 Harbor Ave.

Memphis, TN

38113

PHONE: (901) 775-2345

$\begin{array}{ll}\square \text { TREE } & \square \text { LOCAL TREE } \\ \square \text { SHRUB } & \square \text { LOCAL SHRUB } \\ \nabla \text { GRASS } & \square \text { LOCAL GRASS } \\ \square \text { FORBS } & \square \text { LOCAL FORBS } \\ \square \text { WETLAND } & \square \text { LOCAL WETLAND }\end{array}$

URL: none currently available

Trees By Touliatos

2020 Brooks Rd.

Memphis, TN

38116

PHONE: (901) 346-8065

$\begin{array}{ll}\nabla \text { TREE } & \nabla \text { LOCAL TREE } \\ \nabla \text { SHRUB } & \nabla \text { LOCAL SHRUB } \\ \nabla \text { GRASS } & \square \text { LOCAL GRASS } \\ \nabla \text { FORBS } & \square \text { LOCAL FORBS } \\ \nabla \text { WETLAND } & \square \text { LOCAL WETLAND }\end{array}$

URL: www.waterplants.net

Nashville

Green Seed Co. Inc.

120 Donelson Pike Suite 102

Nashville, TN

37214

PHONE: (615) 883-2554

$\begin{array}{ll}\square \text { TREE } & \square \text { LOCAL TREE } \\ \square \text { SHRUB } & \square \text { LOCAL SHRUB } \\ \nabla \text { GRASS } & \square \text { LOCAL GRASS } \\ \square \text { FORBS } & \square \text { LOCAL FORBS } \\ \square \text { WETLAND } & \square \text { LOCAL WETLAND }\end{array}$

URL: none currently available

\section{Rock Island}

Pleasant Cove Nursery

2400 Old Rock Island Rd.

Rock Island, TN

38581

PHONE: (931) 686-2215

$\begin{array}{ll}\nabla \text { TREE } & \nabla \text { LOCAL TREE } \\ \nabla \text { SHRUB } & \square \text { LOCAL SHRUB } \\ \square \text { GRASS } & \square \text { LOCAL GRASS } \\ \square \text { FORBS } & \square \text { LOCAL FORBS } \\ \square \text { WETLAND } & \square \text { LOCAL WETLAND }\end{array}$

URL: none currently available

Triple W Nursery

917 Hennessee Bridge Rd

Rock Island, TN

38581

PHONE: (931) 686-8393

$\begin{array}{ll}\nabla \text { TREE } & \square \text { LOCAL TREE } \\ \nabla \text { SHRUB } & \square \text { LOCAL SHRUB } \\ \square \text { GRASS } & \square \text { LOCAL GRASS } \\ \square \text { FORBS } & \square \text { LOCAL FORBS } \\ \square \text { WETLAND } & \square \text { LOCAL WETLAND }\end{array}$

URL: none currently available 
Smartt

Flower City Nurseries

PO Box 75

Smartt, TN

37378

PHONE: (931) 668-4351

$\begin{array}{ll}\nabla \text { TREE } & \square \text { LOCAL TREE } \\ \nabla \text { SHRUB } & \square \text { LOCAL SHRUB } \\ \square \text { GRASS } & \square \text { LOCAL GRASS } \\ \square \text { FORBS } & \square \text { LOCAL FORBS } \\ \square \text { WETLAND } & \square \text { LOCAL WETLAND }\end{array}$

URL: http://Www thnursery.com/FlowerCityl

\section{Smithville}

Center Hill Nursery

897 South Congress Blvd.

$\nabla$ TREE $\quad \nabla$ LOCAL TREE

Smithville, TN

37166

$\checkmark$ SHRUB

$\checkmark$ LOCAL SHRUB

PHONE: (615) 597-1888

$\square$ GRASS $\square$ LOCAL GRASS

$\square$ FORBS $\square$ LOCAL FORBS

$\square$ WETLAND $\square$ LOCAL WETLAND

URL: http:/Wwww.centerhillnursery.uS/

Judkins Nursery

6416 Short Mountain Hwy

Smithville, TN

37166

PHONE: (615) 597-7215

$\begin{array}{ll}\nabla \text { TREE } & \nabla \text { LOCAL TREE } \\ \nabla \text { SHRUB } & \nabla \text { LOCAL SHRUB } \\ \square \text { GRASS } & \square \text { LOCAL GRASS } \\ \square \text { FORBS } & \square \text { LOCAL FORBS } \\ \square \text { WETLAND } & \square \text { LOCAL WETLAND }\end{array}$

URL: none currently available

\section{Tullahoma}

Shahan Bros. Nursery

PO Box 876

Tullahoma, TN

37388

PHONE: (931) 455-3297

$\begin{array}{ll}\nabla \text { TREE } & \square \text { LOCAL TREE } \\ \square \text { SHRUB } & \nabla \text { LOCAL SHRUB } \\ \square \text { GRASS } & \square \text { LOCAL GRASS } \\ \square \text { FORBS } & \square \text { LOCAL FORBS } \\ \square \text { WETLAND } & \square \text { LOCAL WETLAND }\end{array}$

URL: http:/Wwww.tnnursery.com/Shahan/

Turtletown

Apalachee Nursery

1210 Kimsey Dairy Road

Turtletown, TN

37391

PHONE: (423) 496-7246

$\begin{array}{ll}\nabla \text { TREE } & \square \text { LOCAL TREE } \\ \nabla \text { SHRUB } & \square \text { LOCAL SHRUB } \\ \square \text { GRASS } & \square \text { LOCAL GRASS } \\ \square \text { FORBS } & \square \text { LOCAL FORBS } \\ \square \text { WETLAND } & \square \text { LOCAL WETLAND }\end{array}$

URL: none currently available

\section{Winchester}

\section{Shadow Nursery}

254 Shadow Nursery Rd.

37398

PHONE: (931) 967-6059

URL: none currently available $\checkmark$ TREE

$\checkmark$ SHRUB

$\begin{array}{ll}\square \text { GRASS } & \square \text { LOCAL GRASS } \\ \square \text { FORBS } & \square \text { LOCAL FORBS } \\ \square \text { WETLAND } & \square \text { LOCAL WETLAND }\end{array}$

$\checkmark$ LOCAL TREE

$\checkmark$ LOCAL SHRUB

$\square$ LOCAL GRASS

$\square$ LOCAL WETLAND 
Texas

Abernathy

Frontier Hybrids

Box 177

Abernathy, TX

79311

PHONE: (806) 298-2595

$\begin{array}{ll}\square \text { TREE } & \square \text { LOCAL TREE } \\ \square \text { SHRUB } & \square \text { LOCAL SHRUB } \\ \square \text { GRASS } & \square \text { LOCAL GRASS } \\ \square \text { FORBS } & \square \text { LOCAL FORBS } \\ \square \text { WETLAND } & \square \text { LOCAL WETLAND }\end{array}$

URL: none currently available

Abilene

Abilene Ag Service \& Supply, Inc.

1301 S. Treadway

Abilene, TX

79602

PHONE: (915) 677-4371

$\begin{array}{ll}\square \text { TREE } & \square \text { LOCAL TREE } \\ \square \text { SHRUB } & \square \text { LOCAL SHRUB } \\ \square \text { GRASS } & \square \text { LOCAL GRASS } \\ \square \text { FORBS } & \square \text { LOCAL FORBS } \\ \square \text { WETLAND } & \square \text { LOCAL WETLAND }\end{array}$

URL: none currently available

Alto

Texas Forest Service

PO Box 617

$\begin{array}{ll}\nabla \text { TREE } & \square \text { LOCAL TREE } \\ \square \text { SHRUB } & \square \text { LOCAL SHRUB } \\ \square \text { GRASS } & \square \text { LOCAL GRASS } \\ \square \text { FORBS } & \square \text { LOCAL FORBS } \\ \square \text { WETLAND } & \square \text { LOCAL WETLAND }\end{array}$

Alto, $T X$

75925

PHONE: (936) 858-4202

$\nabla$ WETLAND $\nabla$ LOCAL WETLAND

URL: http://txforestservice.tamu.edu/

Arlington

Bluestem Nurseries

4101 Curry Rd.

Arlington, TX

76001

$\square$ TREE

$\square$ SHRUB

$\checkmark$ GRASS

$\square$ LOCAL TREE

$\square$ FORBS

$\square$ LOCAL SHRUB

PHONE: (817) 478-6202

$\checkmark$ WETLAND

$\checkmark$ LOCAL GRASS

$\square$ LOCAL FORBS

URL: none currently available

Austin

Barton Springs Nursery

3601 Bee Cave Rd

Austin, TX

78746

PHONE: (512) 328-6655

$\begin{array}{ll}\nabla \text { TREE } & \square \text { LOCAL TREE } \\ \nabla \text { SHRUB } & \square \text { LOCAL SHRUB } \\ \nabla \text { GRASS } & \square \text { LOCAL GRASS } \\ \nabla \text { FORBS } & \square \text { LOCAL FORBS } \\ \square \text { WETLAND } & \square \text { LOCAL WETLAND }\end{array}$

URL: none currently available

Gardens

1818 W. 35th St.

$\nabla$ TREE

$\checkmark$ SHRUB

$\nabla$ GRASS

78703

$\checkmark$ FORBS

$\square$ WETLAND

$\square$ LOCAL TREE

$\square$ LOCAL SHRUB

$\square$ LOCAL GRASS

$\square$ LOCAL FORBS

PHONE: (512) 451-5490

$\square$ LOCAL WETLAND

URL: www.gardens-austin.com 
John Deere Landscapes

10805 Metric Boulevard

Austin, TX

$\checkmark$ TREE $\quad \square$ LOCAL TREE

78758

PHONE: (512) 833-5070

$\nabla$ SHRUB $\square$ LOCAL SHRUB

$\square$ GRASS $\square$ LOCAL GRASS

$\checkmark$ FORBS $\square$ LOCAL FORBS

$\square$ WETLAND $\square$ LOCAL WETLAND

URL: www.johndeerelandscapes.com

Texzen Gardens

4806 Burnet Rd

Austin, TX

78756

PHONE: (512) 454-6471

$\begin{array}{ll}\square \text { TREE } & \square \text { LOCAL TREE } \\ \square \text { SHRUB } & \square \text { LOCAL SHRUB } \\ \square \text { GRASS } & \square \text { LOCAL GRASS } \\ \square \text { FORBS } & \square \text { LOCAL FORBS } \\ \square \text { WETLAND } & \square \text { LOCAL WETLAND }\end{array}$

URL: none currently available

\section{Breckenridge}

Turner Seed Co., Inc.

211 CR 151

Breckenridge, TX

76024

PHONE: (817) 559-2065

$\begin{array}{ll}\square \text { TREE } & \square \text { LOCAL TREE } \\ \square \text { SHRUB } & \square \text { LOCAL SHRUB } \\ \nabla \text { GRASS } & \square \text { LOCAL GRASS } \\ \nabla \text { FORBS } & \square \text { LOCAL FORBS } \\ \square \text { WETLAND } & \square \text { LOCAL WETLAND }\end{array}$

URL: none currently available

\section{Comfort}

Atkison Seed \& Supply

Box 656

Comfort, TX

78013

PHONE: (830) 995-2186

$\begin{array}{ll}\square \text { TREE } & \square \text { LOCAL TREE } \\ \square \text { SHRUB } & \square \text { LOCAL SHRUB } \\ \square \text { GRASS } & \square \text { LOCAL GRASS } \\ \square \text { FORBS } & \square \text { LOCAL FORBS } \\ \square \text { WETLAND } & \square \text { LOCAL WETLAND }\end{array}$

URL: none currently available

\section{El Paso}

Plantation Nursery

105 Lindbergh

El Paso, TX

79932

PHONE: (915) 584-0433

$\begin{array}{ll}\nabla \text { TREE } & \square \text { LOCAL TREE } \\ \square \text { SHRUB } & \square \text { LOCAL SHRUB } \\ \square \text { GRASS } & \square \text { LOCAL GRASS } \\ \nabla \text { FORBS } & \square \text { LOCAL FORBS } \\ \square \text { WETLAND } & \square \text { LOCAL WETLAND }\end{array}$

URL: none currently available

\section{Fredericksburg}

Wildseed Farms Incorporated

PO Box 3000

Fredericksburg, TX

78624

PHONE: (830) 990-8080

$\begin{array}{ll}\square \text { TREE } & \square \text { LOCAL TREE } \\ \square \text { SHRUB } & \square \text { LOCAL SHRUB } \\ \square \text { GRASS } & \square \text { LOCAL GRASS } \\ \square \text { FORBS } & \square \text { LOCAL FORBS } \\ \square \text { WETLAND } & \square \text { LOCAL WETLAND }\end{array}$

URL: www.wildseedfarms.com 
Houston

Treesearch Farm

7625 Alabonson Rd.

Houston, TX

77088

PHONE: (713) 937-9811

$\begin{array}{ll}\nabla \text { TREE } & \square \text { LOCAL TREE } \\ \nabla \text { SHRUB } & \square \text { LOCAL SHRUB } \\ \nabla \text { GRASS } & \square \text { LOCAL GRASS } \\ \square \text { FORBS } & \square \text { LOCAL FORBS } \\ \square \text { WETLAND } & \square \text { LOCAL WETLAND }\end{array}$

URL: www.treesearchfarms.com

Junction

Native American Seed

127 N. 16 th St.

Junction, TX

76849

PHONE: (325) 446-3600

$\begin{array}{ll}\square \text { TREE } & \square \text { LOCAL TREE } \\ \square \text { SHRUB } & \square \text { LOCAL SHRUB } \\ \square \text { GRASS } & \square \text { LOCAL GRASS } \\ \square \text { FORBS } & \square \text { LOCAL FORBS } \\ \square \text { WETLAND } & \square \text { LOCAL WETLAND }\end{array}$

URL: www.seedsource.com

Justin

Justin Seed Co.

524 S. Highway 156 Box 6

Justin, TX

76247

PHONE: (940) 648-2751

$\begin{array}{ll}\square \text { TREE } & \square \text { LOCAL TREE } \\ \square \text { SHRUB } & \square \text { LOCAL SHRUB } \\ \square \text { GRASS } & \square \text { LOCAL GRASS } \\ \square \text { FORBS } & \square \text { LOCAL FORBS } \\ \square \text { WETLAND } & \square \text { LOCAL WETLAND }\end{array}$

URL: www.justinseed.com

\section{Kenedy}

Pogue Agri Partners

PO Box 389

Kenedy, TX

78119

PHONE: (830) 583-3456

$\begin{array}{ll}\square \text { TREE } & \square \text { LOCAL TREE } \\ \square \text { SHRUB } & \square \text { LOCAL SHRUB } \\ \square \text { GRASS } & \square \text { LOCAL GRASS } \\ \square \text { FORBS } & \square \text { LOCAL FORBS } \\ \square \text { WETLAND } & \square \text { LOCAL WETLAND }\end{array}$

URL: www.pogueagri.com

\section{Kingsville}

Plant Mlaterials Center

3409 N FM 1355

Kingsville, TX

78363

PHONE: (361) 595-1313

$\begin{array}{ll}\square \text { TREE } & \square \text { LOCAL TREE } \\ \square \text { SHRUB } & \square \text { LOCAL SHRUB } \\ \square \text { GRASS } & \square \text { LOCAL GRASS } \\ \square \text { FORBS } & \square \text { LOCAL FORBS } \\ \square \text { WETLAND } & \square \text { LOCAL WETLAND }\end{array}$

URL: http://plant-materials.nrcs.usda.gov/stpmc/

\section{Knox City}

Plant Materials Center

3776 Farm Rd 1292

Knox City, TX

79529

PHONE: (940) 658-3922

$\begin{array}{ll}\square \text { TREE } & \square \text { LOCAL TREE } \\ \square \text { SHRUB } & \square \text { LOCAL SHRUB } \\ \square \text { GRASS } & \square \text { LOCAL GRASS } \\ \square \text { FORBS } & \square \text { LOCAL FORBS } \\ \square \text { WETLAND } & \square \text { LOCAL WETLAND }\end{array}$

URL: http://plant-materials.nrcs.usda.govftxpmc/ 
Lewisville

John Deere Landscapes, Inc.

11120 S. Texas Rd

Lewisville, $T X$

75057

PHONE: (972) 219-1509

$\begin{array}{ll}\nabla \text { TREE } & \square \text { LOCAL TREE } \\ \nabla \text { SHRUB } & \square \text { LOCAL SHRUB } \\ \nabla \text { GRASS } & \square \text { LOCAL GRASS } \\ \nabla \text { FORBS } & \square \text { LOCAL FORBS } \\ \square \text { WETLAND } & \square \text { LOCAL WETLAND }\end{array}$

URL: www.johndeerelandscapes.com

\section{Lubbock}

Richardson Seeds Ltd.

3908 N. Frankfort Rd

Lubbock, TX

79416

$\square$ SHRUB $\quad \square$ LOCAL SHRUB

PHONE: (806) 749-2247

$\square$ GRASS $\square$ LOCAL GRASS

$\begin{array}{ll}\square \text { FORBS } & \square \text { LOCAL FORBS } \\ \square \text { WETLAND } & \square \text { LOCAL WETLAND }\end{array}$

URL: none currently available

Maple

High Plains Native Grass Seed

Box 69

Maple, TX

79344

PHONE: (806) 927-5545

$\begin{array}{ll}\square \text { TREE } & \square \text { LOCAL TREE } \\ \square \text { SHRUB } & \square \text { LOCAL SHRUB } \\ \square \text { GRASS } & \square \text { LOCAL GRASS } \\ \square \text { FORBS } & \square \text { LOCAL FORBS } \\ \square \text { WETLAND } & \square \text { LOCAL WETLAND }\end{array}$

URL: none currently available

Mason

Dewey Eckert

Brady Rt., Box 27"

Mason, TX

76856

PHONE: (325) 258-4441

$\begin{array}{ll}\square \text { TREE } & \square \text { LOCAL TREE } \\ \square \text { SHRUB } & \square \text { LOCAL SHRUB } \\ \square \text { GRASS } & \square \text { LOCAL GRASS } \\ \square \text { FORBS } & \square \text { LOCAL FORBS } \\ \square \text { WETLAND } & \square \text { LOCAL WETLAND }\end{array}$

URL: none currently available

Maxwell

Tangram Nursery

5315 Tangram Nursery Rd.

Maxwell, TX

78656

PHONE: (512) 396-0667

$\begin{array}{ll}\square \text { TREE } & \square \text { LOCAL TREE } \\ \square \text { SHRUB } & \square \text { LOCAL SHRUB } \\ \square \text { GRASS } & \square \text { LOCAL GRASS } \\ \square \text { FORBS } & \square \text { LOCAL FORBS } \\ \square \text { WETLAND } & \square \text { LOCAL WETLAND }\end{array}$

URL: none currently available

\section{Montgomery}

Number 1 Nursery Tree Farm

131 Raborn Chapel Rd.

Montgomery, TX

77356

PHONE: (936) 588-2508

$\begin{array}{ll}\nabla \text { TREE } & \square \text { LOCAL TREE } \\ \nabla \text { SHRUB } & \square \text { LOCAL SHRUB } \\ \square \text { GRASS } & \square \text { LOCAL GRASS } \\ \square \text { FORBS } & \square \text { LOCAL FORBS } \\ \square \text { WETLAND } & \square \text { LOCAL WETLAND }\end{array}$

URL: none currently available 
Muleshoe

Bamert Seed Co.

Rt.3, Box 1120

Muleshoe, TX

79347

PHONE: (806) 272-5506

URL: www.bamertseed.com

McCormick Seeds, Inc.

400 E. Ash

Muleshoe, TX

79347

PHONE: (806) 272-3156

URL: none currently available

\section{Nacogdoches}

Lone Star Farm \& Home Center

608 W. Main

Nacogdoches, TX

75961

PHONE: (936) 560-8377

URL: none currently available

Plant Materials Center

6598 FM2782

Nacogdoches, TX

75964

PHONE: (936) 564-4873

URL: http://plant-materials.nrcs.usda.gov/etpmc/

\section{Plainview}

Browning Seed

Box 1836

Plainview, TX

79072

PHONE: (800) 243-5271

$\begin{array}{ll}\square \text { TREE } & \square \text { LOCAL TREE } \\ \square \text { SHRUB } & \square \text { LOCAL SHRUB } \\ \square \text { GRASS } & \square \text { LOCAL GRASS } \\ \square \text { FORBS } & \square \text { LOCAL FORBS } \\ \square \text { WETLAND } & \square \text { LOCAL WETLAND }\end{array}$

$\begin{array}{ll}\square \text { TREE } & \square \text { LOCAL TREE } \\ \square \text { SHRUB } & \square \text { LOCAL SHRUB } \\ \square \text { GRASS } & \square \text { LOCAL GRASS } \\ \nabla \text { FORBS } & \square \text { LOCAL FORBS } \\ \square \text { WETLAND } & \square \text { LOCAL WETLAND }\end{array}$

SHRUB

$\nabla$ GRASS

$\checkmark$ FORBS

$\square$ WETLAND

$\square$ LOCAL TREE

$\square$ LOCAL SHRUB

$\square$ LOCAL GRASS

$\square$ LOCAL FORBS

$\square$ LOCAL WETLAND

$\begin{array}{ll}\square \text { TREE } & \square \text { LOCAL TREE } \\ \square \text { SHRUB } & \square \text { LOCAL SHRUB } \\ \square \text { GRASS } & \square \text { LOCAL GRASS } \\ \square \text { FORBS } & \square \text { LOCAL FORBS } \\ \square \text { WETLAND } & \square \text { LOCAL WETLAND }\end{array}$

URL: www.browningseed.com

\section{Richmond}

Sunbelt Trees, Inc.

16008 Boss Gaston

Richmond, TX

77469

PHONE: (800) 635-4313

$\begin{array}{ll}\nabla \text { TREE } & \square \text { LOCAL TREE } \\ \square \text { SHRUB } & \square \text { LOCAL SHRUB } \\ \square \text { GRASS } & \square \text { LOCAL GRASS } \\ \square \text { FORBS } & \square \text { LOCAL FORBS } \\ \square \text { WETLAND } & \square \text { LOCAL WETLAND }\end{array}$

URL: www.sunbelttrees.com 


\section{San Angelo}

Schertz Landscape $\mathrm{C}_{0}$.

2225 Knickerbocker Rd

San Angelo, TX

76904

PHONE: (325) 944-0511

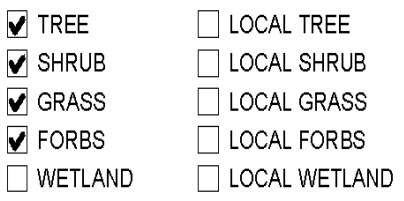

URL: http://www scherzlandscape.com

\section{San Antonio}

Douglass W. King Seed Co.

Box 200320

San Antonio, TX

78220

$\square$ SHRUB $\quad \square$ LOCAL SHRUB

PHONE: (210) 661-4191

$\square$ GRASS $\square$ LOCAL GRASS

$\begin{array}{ll}\square \text { FORBS } & \square \text { LOCAL FORBS } \\ \square \text { WETLAND } & \square \text { LOCAL WETLAND }\end{array}$

URL: www.dkseeds.com

Shamrock

James Reneau Seed Co.

119 S. Main St.

Shamrock, TX

79079

PHONE: (806) 256-3216

$\begin{array}{ll}\square \text { TREE } & \square \text { LOCAL TREE } \\ \square \text { SHRUB } & \square \text { LOCAL SHRUB } \\ \square \text { GRASS } & \square \text { LOCAL GRASS } \\ \square \text { FORBS } & \square \text { LOCAL FORBS } \\ \square \text { WETLAND } & \square \text { LOCAL WETLAND }\end{array}$

URL: none currently available

\section{Spearman}

Watley Seed Co.

Box 51

Spearman, TX

79081

PHONE: (806) 659-3838

$\begin{array}{ll}\square \text { TREE } & \square \text { LOCAL TREE } \\ \square \text { SHRUB } & \square \text { LOCAL SHRUB } \\ \square \text { GRASS } & \square \text { LOCAL GRASS } \\ \square \text { FORBS } & \square \text { LOCAL FORBS } \\ \square \text { WETLAND } & \square \text { LOCAL WETLAND }\end{array}$

URL: none currently available

Uvalde

Foster-Rambie Grass Seed

PO Box 5386

Uvalde, TX

78802

PHONE: (830) 278-2711

$\begin{array}{ll}\square \text { TREE } & \square \text { LOCAL TREE } \\ \square \text { SHRUB } & \square \text { LOCAL SHRUB } \\ \square \text { GRASS } & \square \text { LOCAL GRASS } \\ \square \text { FORBS } & \square \text { LOCAL FORBS } \\ \square \text { WETLAND } & \square \text { LOCAL WETLAND }\end{array}$

URL: none currently available

\begin{tabular}{|c|c|c|}
\hline Van Ormy & & \\
\hline Aldridge Nursery & & \\
\hline PO Box 1299 & $\checkmark$ TREE & $\checkmark$ LOCAL TREE \\
\hline Van Ormy, TX & $\bar{V}$ SHRUB & $\bar{\nabla}$ LOCAL SHRUB \\
\hline 78073 & $\square$ GRASS & $\square$ LOCAL GRASS \\
\hline PHONE: (210) 622-3491 & $\square$ FORBS & $\square$ LOCAL FORBS \\
\hline & $\square$ WETLAND & $\square$ LOCAL WETLAND \\
\hline
\end{tabular}

URL: www.aldridgenursery.com 
Vernon

Texas Foundation Seed Service

11914 Hwy 70 S.

Vernon, TX

76384

PHONE: (940) 552-6226

$\begin{array}{ll}\square \text { TREE } & \square \text { LOCAL TREE } \\ \square \text { SHRUB } & \square \text { LOCAL SHRUB } \\ \square \text { GRASS } & \square \text { LOCAL GRASS } \\ \square \text { FORBS } & \square \text { LOCAL FORBS } \\ \square \text { WETLAND } & \square \text { LOCAL WETLAND }\end{array}$

URL: http://tiss.tamu.edu/

Warren

Doremus Wholesale Nursery

Rt.2, Box 750

$\nabla$ TREE $\quad \nabla$ LOCAL TREE

Warren, TX

$\checkmark$ SHRUB

$\nabla$ LOCAL SHRUB

77664

$\square$ GRASS

$\square$ LOCAL GRASS

PHONE: (409) 547-3536

$\square$ FORBS $\quad \square$ LOCAL FORBS

$\square$ WETLAND $\square$ LOCAL WETLAND

URL: www.doremusnursery.com

\section{Utah}

Draper

Lone Peak Nursery

$\begin{array}{lll}271 \text { Bitterbrush Ln } & \nabla \text { TREE } & \nabla \text { LOCAL TREE } \\ \text { Draper, UT } & \nabla \text { SHRUB } & \nabla \text { LOCAL SHRUB } \\ \text { 84020-959 } & \nabla \text { GRASS } & \nabla \text { LOCAL GRASS } \\ \text { PHONE: (801) 571-0900 } & \nabla \text { FORBS } & \nabla \text { LOCAL FORBS } \\ & \nabla \text { WETLAND } & \nabla \text { LOCAL WETLAND }\end{array}$

URL: http://Ww'w.ffsl.utah.gov/lonepeak/nursery/LPNursery.htm

\section{Ephraim}

Intermountain Seed

300 W. $300 \mathrm{~N}$.

Ephraim, UT

84627

PHONE: (435) 283-4703

$\begin{array}{ll}\square \text { TREE } & \square \text { LOCAL TREE } \\ \square \text { SHRUB } & \square \text { LOCAL SHRUB } \\ \square \text { GRASS } & \square \text { LOCAL GRASS } \\ \square \text { FORBS } & \square \text { LOCAL FORBS } \\ \square \text { WETLAND } & \square \text { LOCAL WETLAND }\end{array}$

URL: none currently available

Maple Leaf Industries

480 S. 50E, Box 9-6"

Ephraim, UT

84627

PHONE: (435) 283-4701

$\begin{array}{ll}\square \text { TREE } & \square \text { LOCAL TREE } \\ \nabla \text { SHRUB } & \square \text { LOCAL SHRUB } \\ \nabla \text { GRASS } & \square \text { LOCAL GRASS } \\ \nabla \text { FORBS } & \square \text { LOCAL FORBS } \\ \nabla \text { WETLAND } & \nabla \text { LOCAL WETLAND }\end{array}$

URL: www.mapleleafinc.com/seed

Plummer Plant \& Seed Co.

Box 70

Ephraim, UT

84627

PHONE: (435) 283-4844

$\begin{array}{ll}\square \text { TREE } & \square \text { LOCAL TREE } \\ \square \text { SHRUB } & \square \text { LOCAL SHRUB } \\ \nabla \text { GRASS } & \square \text { LOCAL GRASS } \\ \square \text { FORBS } & \square \text { LOCAL FORBS } \\ \square \text { WETLAND } & \square \text { LOCAL WETLAND }\end{array}$

URL: none currently available 
Stevenson Intermountain Seed

Box 2

Ephraim, UT

84627

PHONE: (435) 283-6639

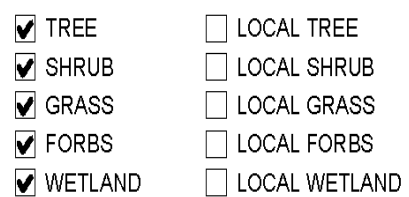

URL: www.stevensonintermountainseed.com

\section{Gunnison}

Goble Seed Co.

Box 203

Gunnison, UT

84634

PHONE: (435) 528-3234

$\begin{array}{ll}\square \text { TREE } & \square \text { LOCAL TREE } \\ \square \text { SHRUB } & \square \text { LOCAL SHRUB } \\ \square \text { GRASS } & \square \text { LOCAL GRASS } \\ \square \text { FORBS } & \square \text { LOCAL FORBS } \\ \square \text { WETLAND } & \square \text { LOCAL WETLAND }\end{array}$

URL: none currently available

Lehi

Granite Seed

1697 West $2100 \mathrm{~N}$

Lehi, UT

84043

PHONE: (801) 768-4422

$\begin{array}{ll}\nabla \text { TREE } & \square \text { LOCAL TREE } \\ \nabla \text { SHRUB } & \square \text { LOCAL SHRUB } \\ \nabla \text { GRASS } & \square \text { LOCAL GRASS } \\ \nabla \text { FORBS } & \square \text { LOCAL FORBS } \\ \nabla \text { WETLAND } & \square \text { LOCAL WETLAND }\end{array}$

URL: wWW.graniteseed.com

\section{Salt Lake City}

Mountain Valley Seeds

1800 SW Temple

Salt Lake City, UT

84115

PHONE: (801) 486-0480

$\begin{array}{ll}\square \text { TREE } & \square \text { LOCAL TREE } \\ \square \text { SHRUB } & \square \text { LOCAL SHRUB } \\ \square \text { GRASS } & \square \text { LOCAL GRASS } \\ \square \text { FORBS } & \square \text { LOCAL FORBS } \\ \square \text { WETLAND } & \square \text { LOCAL WETLAND }\end{array}$

URL: www.mvseeds.com

Sandy

Biograss Farms

9980 South State

Sandy. UT

84070

PHONE: (801) 562-9090

$\begin{array}{ll}\square \text { TREE } & \square \text { LOCAL TREE } \\ \square \text { SHRUB } & \square \text { LOCAL SHRUB } \\ \square \text { GRASS } & \square \text { LOCAL GRASS } \\ \square \text { FORBS } & \square \text { LOCAL FORBS } \\ \square \text { WETLAND } & \square \text { LOCAL WETLAND }\end{array}$

URL: www.biograss.net

Progressive Plants

9180 S. Wasatch Blvd.

Sandy, UT

84093

PHONE: (801) 942-7333

$\begin{array}{ll}\nabla \text { TREE } & \square \text { LOCAL TREE } \\ \nabla \text { SHRUB } & \square \text { LOCAL SHRUB } \\ \nabla \text { GRASS } & \square \text { LOCAL GRASS } \\ \nabla \text { FORBS } & \square \text { LOCAL FORBS } \\ \square \text { WETLAND } & \square \text { LOCAL WETLAND }\end{array}$

URL: www.progressiveplants.com 


\section{Virginia}

Afton

Edible Landscaping

P.O. Box 77

Afton, VA

$\nabla$ TREE

$\checkmark$ SHRUB

22920

PHONE: (434) 361-9134

$\square$ GRASS

$\square$ FORBS $\square$ LOCAL FORBS

URL: http://Www ediblelandscaping.com/

Bristol

Country Boy Seed Co.

6685 Gate City Hwy.

Bristol, VA

24202

$\square$ WETLAND

$$
\begin{aligned}
& \nabla \text { LOCAL TREE } \\
& \nabla \text { LOCAL SHRUB } \\
& \square \text { LOCAL GRASS } \\
& \square \text { LOCAL FORBS } \\
& \square \text { LOCAL WETLAND }
\end{aligned}
$$

PHONE: (276) 669-1004

$\begin{array}{ll}\square \text { TREE } & \square \text { LOCAL TREE } \\ \square \text { SHRUB } & \square \text { LOCAL SHRUB } \\ \square \text { GRASS } & \square \text { LOCAL GRASS } \\ \square \text { FORBS } & \square \text { LOCAL FORBS } \\ \square \text { WETLAND } & \square \text { LOCAL WETLAND }\end{array}$

URL: www.countryboyseed.com

\section{Charlottesville}

Virginia Department of Forestry

900 Natural Resources Drive Suite 800

Charlottesville, VA

22903

PHONE: (434) 977-6555

$\begin{array}{ll}\nabla \text { TREE } & \square \text { LOCAL TREE } \\ \nabla \text { SHRUB } & \square \text { LOCAL SHRUB } \\ \square \text { GRASS } & \square \text { LOCAL GRASS } \\ \square \text { FORBS } & \square \text { LOCAL FORBS } \\ \square \text { WETLAND } & \square \text { LOCAL WETLAND }\end{array}$

URL: http:/NWWW.vdof.org/

\section{Chesapeake}

Greenbrier Farms, Inc.

201 Hickory Rd. W.

Chesapeake, VA

23322

PHONE: (757) 421-2141

$\begin{array}{ll}\nabla \text { TREE } & \nabla \text { LOCAL TREE } \\ \nabla \text { SHRUB } & \square \text { LOCAL SHRUB } \\ \square \text { GRASS } & \square \text { LOCAL GRASS } \\ \square \text { FORBS } & \square \text { LOCAL FORBS } \\ \square \text { WETLAND } & \square \text { LOCAL WETLAND }\end{array}$

URL: none currently available

\section{Gloucester Point}

Coastal Wetlands Nursery, Inc.

$$
\text { P.O. Box } 1018
$$

Gloucester Point, VA

23062

PHONE: (804) 693-2619

$\begin{array}{ll}\square \text { TREE } & \square \text { LOCAL TREE } \\ \square \text { SHRUB } & \square \text { LOCAL SHRUB } \\ \square \text { GRASS } & \square \text { LOCAL GRASS } \\ \square \text { FORBS } & \square \text { LOCAL FORBS } \\ \square \text { WETLAND } & \square \text { LOCAL WETLAND }\end{array}$

URL: none currently available

$\begin{array}{lll}\text { Hume } & & \\ \text { Virginia Natives } & & \\ \text { P.O. Box } 18 & \square \text { TREE } & \square \text { LOCAL TREE } \\ \text { Hume, VA } & \square \text { SHRUB } & \square \text { LOCAL SHRUB } \\ 22639 & \square \text { GRASS } & \square \text { LOCAL GRASS } \\ \text { PHONE: (540) 364-1665 } & \square \text { FORBS } & \square \text { LOCAL FORBS } \\ & \square \text { WETLAND } & \square \text { LOCAL WETLAND }\end{array}$

URL: none currently available 
Lovettsville

Water Ways Nursery

13015 Middletown Rd.

Lovettsville, VA

20180

PHONE: (540) 822-5994

$\begin{array}{ll}\nabla \text { TREE } & \square \text { LOCAL TREE } \\ \square \text { SHRUB } & \nabla \text { LOCAL SHRUB } \\ \square \text { GRASS } & \square \text { LOCAL GRASS } \\ \square \text { FORBS } & \square \text { LOCAL FORBS } \\ \square \text { WETLAND } & \square \text { LOCAL WETLAND }\end{array}$

URL: none currently available

Melfa

Bobtown Nursery

16212 Country Club Rd

Melfa, VA

23410

PHONE: (804) 787-8484

$\begin{array}{ll}\square \text { TREE } & \square \text { LOCAL TREE } \\ \square \text { SHRUB } & \square \text { LOCAL SHRUB } \\ \nabla \text { GRASS } & \square \text { LOCAL GRASS } \\ \nabla \text { FORBS } & \square \text { LOCAL FORBS } \\ \square \text { WETLAND } & \square \text { LOCAL WETLAND }\end{array}$

URL: www.bobtown.com

Mobjack

Mobjack Nurseries, Inc.

H.C. 75 , Box 7965

Mobjack, VA

23056-796

PHONE: (800) 729-6625

$\begin{array}{ll}\nabla \text { TREE } & \square \text { LOCAL TREE } \\ \nabla \text { SHRUB } & \square \text { LOCAL SHRUB } \\ \square \text { GRASS } & \square \text { LOCAL GRASS } \\ \square \text { FORBS } & \square \text { LOCAL FORBS } \\ \square \text { WETLAND } & \square \text { LOCAL WETLAND }\end{array}$

URL: www.mobjack.com

\section{Oak Grove}

Ingleside Plantation Nurseries

Box 1038

Oak Grove, VA

22443

PHONE: (804) 224-7111

$\begin{array}{ll}\nabla \text { TREE } & \square \text { LOCAL TREE } \\ \nabla \text { SHRUB } & \checkmark \text { LOCAL SHRUB } \\ \square \text { GRASS } & \square \text { LOCAL GRASS } \\ \square \text { FORBS } & \square \text { LOCAL FORBS } \\ \square \text { WETLAND } & \square \text { LOCAL WETLAND }\end{array}$

URL: http://Www inglesidenurseries.com/

\section{Roanoake}

Landscape Supply

Box 12706

Roanoake. VA

24027

PHONE: (800) 876-2151

WWw.landscapesupplyonline.com

$\begin{array}{ll}\square \text { TREE } & \square \text { LOCAL TREE } \\ \square \text { SHRUB } & \square \text { LOCAL SHRUB } \\ \square \text { GRASS } & \square \text { LOCAL GRASS } \\ \square \text { FORBS } & \square \text { LOCAL FORBS } \\ \square \text { WETLAND } & \square \text { LOCAL WETLAND }\end{array}$

\section{Waynesboro}

Waynesboro Nurseries, Inc.

Box 987

Waynesboro, VA

22980

PHONE: (540) 942-4141

$\begin{array}{ll}\nabla \text { TREE } & \square \text { LOCAL TREE } \\ \square \text { SHRUB } & \square \text { LOCAL SHRUB } \\ \square \text { GRASS } & \square \text { LOCAL GRASS } \\ \square \text { FORBS } & \square \text { LOCAL FORBS } \\ \square \text { WETLAND } & \square \text { LOCAL WETLAND }\end{array}$

URL: http:/WwWw.waynesboronurseries.com 


\section{Vermont}

Williston

American Meadows / Vermont Wildflower Farm

$\begin{array}{lll}223 \text { Ave. D } \# 30 & \square \text { TREE } & \square \text { LOCAL TREE } \\ \text { Williston, } V T & \square \text { SHRUB } & \square \text { LOCAL SHRUB } \\ 05495 & \square \text { GRASS } & \square \text { LOCAL GRASS } \\ \text { PHONE: (802) } 951-5812 & \square \text { FORBS } & \square \text { LOCAL FORBS } \\ & \square \text { WETLAND } & \square \text { LOCAL WETLAND }\end{array}$

URL: www.americanmeadows.com

\section{Washington}

Battle Ground

Viewcrest Nurseries, Inc.

12713 NE 184th St.

Battle Ground, WA

98604

PHONE: (360) 687-5167

$\begin{array}{ll}\square \text { TREE } & \square \text { LOCAL TREE } \\ \square \text { SHRUB } & \checkmark \text { LOCAL SHRUB } \\ \square \text { GRASS } & \square \text { LOCAL GRASS } \\ \square \text { FORBS } & \square \text { LOCAL FORBS } \\ \square \text { WETLAND } & \square \text { LOCAL WETLAND }\end{array}$

URL: http:/Wwww.viewcrest.com/

$\begin{array}{lll}\begin{array}{l}\text { Bellingham } \\ \text { Fourth Corner Nurseries }\end{array} & \\ \text { 3057 E. Bakerview Rd. } & \nabla \text { TREE } & \nabla \text { LOCAL TREE } \\ \text { Bellingham. WA } & \nabla \text { SHRUB } & \nabla \text { LOCAL SHRUB } \\ 98226 & \nabla \text { GRASS } & \nabla \text { LOCAL GRASS } \\ \text { PHONE: (360) 734-0079 } & \nabla \text { FORBS } & \nabla \text { LOCAL FORBS } \\ & \nabla \text { WETLAND } & \nabla \text { LOCAL WETLAND }\end{array}$

URL: www.4th-corner-nurseries.com

Bow

WACD Plant Materials Center

16564 Bradley Rd.

Bow, WA

98233

PHONE: (360) 757-1094

$\begin{array}{ll}\nabla \text { TREE } & \square \text { LOCAL TREE } \\ \nabla \text { SHRUB } & \square \text { LOCAL SHRUB } \\ \square \text { GRASS } & \square \text { LOCAL GRASS } \\ \square \text { FORBS } & \square \text { LOCAL FORBS } \\ \square \text { WETLAND } & \square \text { LOCAL WETLAND }\end{array}$

URL: http://Www.wacd.org/PMC/PMC.htm

Buckley

Buckley Nursery $\mathrm{C}_{0}$.

$646 \mathrm{~N}$. River Rd

Buckley, WA

98321

PHONE: (360) 829-1811

$\begin{array}{ll}\nabla \text { TREE } & \nabla \text { LOCAL TREE } \\ \nabla \text { SHRUB } & \nabla \text { LOCAL SHRUB } \\ \square \text { GRASS } & \square \text { LOCAL GRASS } \\ \square \text { FORBS } & \square \text { LOCAL FORBS } \\ \square \text { WETLAND } & \square \text { LOCAL WETLAND }\end{array}$

URL: none currently available

\section{Clarkston}

Grassland West Co.

908 Port Dr.

Clarkston. WA

99403

$\square$ TREE

$\square$ SHRUB

$\checkmark$ GRASS

PHONE: (509) 758-9100

$\square$ FORBS

$\square$ LOCAL TREE

$\square$ LOCAL SHRUB

PHONE: (509) 758-9100

$\square$ WETLAND

$\square$ LOCAL GRASS

$\square$ LOCAL FORBS

URL: http://Ww'.grasslandwest.com/ 
Colfax

Colfax Grange Supply

E. 105 Harrison St

Colfax, WA

99111

$\checkmark$ TREE

$\checkmark$ SHRUB

$\checkmark$ GRASS

$\checkmark$ FORBS

PHONE: (509) 397-4324

$\square$ WETLAND $\square$ LOCAL WETLAND

URL: www.colfaxgrange.com

Connell

L\&H Seeds, Inc.

4756 W. Hwy. 260

Connell, WA

99326

PHONE: (509) 234-4433

$\begin{array}{ll}\square \text { TREE } & \square \text { LOCAL TREE } \\ \square \text { SHRUB } & \square \text { LOCAL SHRUB } \\ \square \text { GRASS } & \square \text { LOCAL GRASS } \\ \nabla \text { FORBS } & \square \text { LOCAL FORBS } \\ \square \text { WETLAND } & \square \text { LOCAL WETLAND }\end{array}$

URL: www.lhseeds.com

\section{Coulee City}

John L. McLean Seed Co.

P.O. Box 815

Coulee City, WA

99115

PHONE: (509) 632-5238

$\begin{array}{ll}\nabla \text { TREE } & \square \text { LOCAL TREE } \\ \nabla \text { SHRUB } & \square \text { LOCAL SHRUB } \\ \nabla \text { GRASS } & \square \text { LOCAL GRASS } \\ \nabla \text { FORBS } & \square \text { LOCAL FORBS } \\ \nabla \text { WETLAND } & \square \text { LOCAL WETLAND }\end{array}$

URL: none currently available

Davenport

Rainier Seeds

1404 Fourth St

$\checkmark$ TREE

$\checkmark$ SHRUB

Davenport, WA

99122

PHONE: (509) 725-1235

$\checkmark$ GRASS

$\checkmark$ FORBS

$\square$ LOCAL SHRUB

$\square$ LOCAL GRASS

$\square$ LOCAL FORBS

$\square$ WETLAND $\square$ LOCAL WETLAND

URL: http://WwW rainierseeds.com/

Des Moines

Furneys Nursery, Inc.

21215 Pacific Hwy. S.

Des Moines, WA

98198

PHONE: (206) 624-0634

$\begin{array}{ll}\nabla \text { TREE } & \square \text { LOCAL TREE } \\ \nabla \text { SHRUB } & \square \text { LOCAL SHRUB } \\ \square \text { GRASS } & \square \text { LOCAL GRASS } \\ \square \text { FORBS } & \square \text { LOCAL FORBS } \\ \nabla \text { WETLAND } & \square \text { LOCAL WETLAND }\end{array}$

URL: http://Www furneysnursery.com

\section{Ellensburg}

Smith-Kem Ellensburg Inc.

P.O. Box 774

$\square$ TREE

Ellensburg, WA

98926

PHONE: (509) 925-5977

$\square$ SHRUB

$\square$ GRASS

$\square$ LOCAL TREE

$\square$ LOCAL SHRUB

$\square$ LOCAL GRASS

$\square$ LOCAL FORBS

$\checkmark$ FORBS

$\square$ LOCAL TREE

$\square$ LOCAL SHRUB

$\square$ LOCAL FORBS

$\square$ WETLAND $\square$ LOCAL WETLAND

URL: www.smithkem.com 


\section{Gold Bear}

Fancy Fronds

P.O. Box 1090

Gold Bear, WA

98251

PHONE: (360) 793-1472

$\begin{array}{ll}\square \text { TREE } & \square \text { LOCAL TREE } \\ \square \text { SHRUB } & \square \text { LOCAL SHRUB } \\ \square \text { GRASS } & \square \text { LOCAL GRASS } \\ \square \text { FORBS } & \square \text { LOCAL FORBS } \\ \square \text { WETLAND } & \square \text { LOCAL WETLAND }\end{array}$

URL: www.fancyfronds.com

Langley

Frosty Hollow Ecological Restoration

PO Box 53

Langley, WA

98260

PHONE: (360) 579-2332

$\begin{array}{ll}\nabla \text { TREE } & \square \text { LOCAL TREE } \\ \square \text { SHRUB } & \square \text { LOCAL SHRUB } \\ \square \text { GRASS } & \square \text { LOCAL GRASS } \\ \nabla \text { FORBS } & \square \text { LOCAL FORBS } \\ \square \text { WETLAND } & \square \text { LOCAL WETLAND }\end{array}$

URL: none currently available

Longview

Watershed Garden Works

2039 - 44th Ave.

Longview, WA

98632

PHONE: (360) 423-6456

$\begin{array}{ll}\nabla \text { TREE } & \square \text { LOCAL TREE } \\ \nabla \text { SHRUB } & \nabla \text { LOCAL SHRUB } \\ \nabla \text { GRASS } & \nabla \text { LOCAL GRASS } \\ \nabla \text { FORBS } & \square \text { LOCAL FORBS } \\ \nabla \text { WETLAND } & \nabla \text { LOCAL WETLAND }\end{array}$

URL: http://www. watershedgardenworks.com/

Lynden

Dewilid Wholesale Nursery

6930 Old Guide Rd.

Lynden, WA

98264

PHONE: (360) 398-1960

$\begin{array}{ll}\square \text { TREE } & \square \text { LOCAL TREE } \\ \nabla \text { SHRUB } & \checkmark \text { LOCAL SHRUB } \\ \square \text { GRASS } & \square \text { LOCAL GRASS } \\ \square \text { FORBS } & \square \text { LOCAL FORBS } \\ \square \text { WETLAND } & \square \text { LOCAL WETLAND }\end{array}$

URL: none currently available

Mesa

Eppich Grain Co.

151 Canal Blvd.

Mesa, WA

99343

PHONE: (509) 269-4693

$\begin{array}{ll}\square \text { TREE } & \square \text { LOCAL TREE } \\ \square \text { SHRUB } & \square \text { LOCAL SHRUB } \\ \square \text { GRASS } & \square \text { LOCAL GRASS } \\ \square \text { FORBS } & \square \text { LOCAL FORBS } \\ \square \text { WETLAND } & \square \text { LOCAL WETLAND }\end{array}$

URL: none currently available

\begin{tabular}{|c|c|c|}
\hline Moses Lake & & \\
\hline Firstline Seeds & & \\
\hline 11703 Rd.1 S.E. & $\square$ TREE & $\square$ LOCAL TREE \\
\hline Moses Lake, WA & $\square$ SHRUB & $\square$ LOCAL SHRUB \\
\hline 98837 & $\checkmark$ GRASS & $\square$ LOCAL GRASS \\
\hline PHONE: (509) 765-1772 & $\square$ FORBS & $\square$ LOCAL FORBS \\
\hline & $\checkmark$ WETLAND & $\square$ LOCAL WETLAND \\
\hline
\end{tabular}

URL: none currently available 
Moses Lake Cons. Dist. Nursery

1775 Southeast Hwy 17

Moses Lake, WA

98837

PHONE: (509) 765-5333

$\begin{array}{ll}\nabla \text { TREE } & \nabla \text { LOCAL TREE } \\ \nabla \text { SHRUB } & \nabla \text { LOCAL SHRUB } \\ \square \text { GRASS } & \square \text { LOCAL GRASS } \\ \square \text { FORBS } & \square \text { LOCAL FORBS } \\ \nabla \text { WETLAND } & \square \text { LOCAL WETLAND }\end{array}$

URL: http://wa.nacdnet.org/mlcd/

Mossyrock

Aldrich Berry Farm \& Nursery

190 Aldrich Rd.

Mossyrock, WA

98564

PHONE: (360) 983-3138

$\begin{array}{ll}\nabla \text { TREE } & \square \text { LOCAL TREE } \\ \nabla \text { SHRUB } & \checkmark \text { LOCAL SHRUB } \\ \square \text { GRASS } & \square \text { LOCAL GRASS } \\ \square \text { FORBS } & \square \text { LOCAL FORBS } \\ \square \text { WETLAND } & \square \text { LOCAL WETLAND }\end{array}$

URL: none currently available

\section{Mt. Vernon}

Christiansons Nursen

15806 Best Rd.

Mt. Vernon, WA

98273

PHONE: (360) 466-3821

$\begin{array}{ll}\nabla \text { TREE } & \square \text { LOCAL TREE } \\ \nabla \text { SHRUB } & \square \text { LOCAL SHRUB } \\ \square \text { GRASS } & \square \text { LOCAL GRASS } \\ \nabla \text { FORBS } & \square \text { LOCAL FORBS } \\ \square \text { WETLAND } & \square \text { LOCAL WETLAND }\end{array}$

URL: hlttp:/Www.christiansonsnursery.com

Olympia

Briggs Nursery

4407 Henderson Blvd.

Olympia, WA

98501

PHONE: (360) 352-5405

$\begin{array}{ll}\nabla \text { TREE } & \square \text { LOCAL TREE } \\ \square \text { SHRUB } & \square \text { LOCAL SHRUB } \\ \square \text { GRASS } & \square \text { LOCAL GRASS } \\ \square \text { FORBS } & \square \text { LOCAL FORBS } \\ \square \text { WETLAND } & \square \text { LOCAL WETLAND }\end{array}$

URL: http://Www briggsnursery.com/

Sound Native Plants

P.O. Box 7505

Olympia, WA

98507

PHONE: (360) 352-4122

$\begin{array}{ll}\nabla \text { TREE } & \nabla \text { LOCAL TREE } \\ \nabla \text { SHRUB } & \nabla \text { LOCAL SHRUB } \\ \nabla \text { GRASS } & \nabla \text { LOCAL GRASS } \\ \nabla \text { FORBS } & \nabla \text { LOCAL FORBS } \\ \nabla \text { WETLAND } & \nabla \text { LOCAL WETLAND }\end{array}$

URL: http://Www. soundnativeplants.com/

Webster Forest Nursery

P.O. Box 47017

Olympia, WA

98504

PHONE: (360) 753-5305

$\begin{array}{ll}\nabla \text { TREE } & \nabla \text { LOCAL TREE } \\ \square \text { SHRUB } & \square \text { LOCAL SHRUB } \\ \square \text { GRASS } & \square \text { LOCAL GRASS } \\ \square \text { FORBS } & \square \text { LOCAL FORBS } \\ \nabla \text { WETLAND } & \square \text { LOCAL WETLAND }\end{array}$

URL: http://www3.wadnr.gov/dnrapp3/webster/index.htm| 


\section{Onalaska}

Burnt Ridge Nursery

432 Burnt Ridge Rd.

Onalaska. WA

98570

PHONE: (360) 985-2873

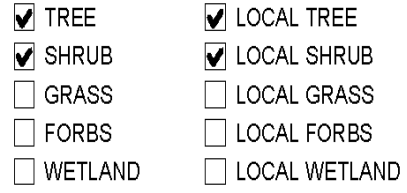

URL: hitp://andru.myhome.net/surntridge/

Pomeroy

Dye Seed Ranch

P.O. Box 610

Pomeroy, WA

99347

$\begin{array}{ll}\square \text { TREE } & \square \text { LOCAL TREE } \\ \square \text { SHRUB } & \square \text { LOCAL SHRUB } \\ \square \text { GRASS } & \square \text { LOCAL GRASS } \\ \square \text { FORBS } & \square \text { LOCAL FORBS } \\ \square \text { WETLAND } & \square \text { LOCAL WETLAND }\end{array}$

PHONE: (509) 291-3661

$\square$ WETLAND $\square$ LOCAL WETLAND

URL: none currently available

Pullman

Plant Materials Center

Hulbert Hall, W.S.U."

Pullman, WA

99164

PHONE: (509) 335-7376

$\begin{array}{ll}\square \text { TREE } & \square \text { LOCAL TREE } \\ \square \text { SHRUB } & \square \text { LOCAL SHRUB } \\ \square \text { GRASS } & \square \text { LOCAL GRASS } \\ \square \text { FORBS } & \square \text { LOCAL FORBS } \\ \square \text { WETLAND } & \square \text { LOCAL WETLAND }\end{array}$

URL: http://plant-materials.nrcs.usda.gov/wapmc/

Roy

Silvaseed Co.

Box 118

Roy, WA

98580

$\nabla$ TREE $\quad \boldsymbol{\nabla}$ LOCAL TREE

$\square$ SHRUB

$\square$ LOCAL SHRUB

PHONE: (253) 843-2246

$\square$ GRASS

$\square$ LOCAL GRASS

$\square$ FORBS

$\square$ LOCAL FORBS

$\square$ WETLAND $\square$ LOCAL WETLAND

URL: hltp://WWw' silvaseed.com/

\section{Seattle}

Rosso Wholesale Nursery

Box 80345

Seattle, WA

98108

PHONE: (206) 763-1888

$\begin{array}{ll}\nabla \text { TREE } & \nabla \text { LOCAL TREE } \\ \nabla \text { SHRUB } & \square \text { LOCAL SHRUB } \\ \square \text { GRASS } & \square \text { LOCAL GRASS } \\ \square \text { FORBS } & \square \text { LOCAL FORBS } \\ \nabla \text { WETLAND } & \square \text { LOCAL WETLAND }\end{array}$

URL: none currently available

\section{Snohomish}

Storm Lake Growers

21809 89th St. S.E.

Snohomish, WA

98290

PHONE: (360) 794-4842

$\begin{array}{ll}\square \text { TREE } & \square \text { LOCAL TREE } \\ \nabla \text { SHRUB } & \square \text { LOCAL SHRUB } \\ \square \text { GRASS } & \square \text { LOCAL GRASS } \\ \square \text { FORBS } & \square \text { LOCAL FORBS } \\ \square \text { WETLAND } & \square \text { LOCAL WETLAND }\end{array}$

URL: http://Www.slgrowers.com/ 


\section{Spokane}

Cascade Seed Wholesale

1406 E. Front Ave

Spokane, WA

99202

PHONE: (509) 534-9431

URL: none currently available

\section{Gibsons Nursery}

S. 1401 Pines Rd.

Spokane, WA

99206

PHONE: (509) 928-0973

$\begin{array}{ll}\square \text { TREE } & \square \text { LOCAL TREE } \\ \square \text { SHRUB } & \square \text { LOCAL SHRUB } \\ \square \text { GRASS } & \square \text { LOCAL GRASS } \\ \square \text { FORBS } & \square \text { LOCAL FORBS } \\ \square \text { WETLAND } & \square \text { LOCAL WETLAND }\end{array}$

URL: none currently available

Northwest Seed \& Pet

E. 2422 Sprague Ave.

Spokane, WA

99202

PHONE: (509) 534-0694

$\begin{array}{ll}\nabla \text { TREE } & \square \text { LOCAL TREE } \\ \nabla \text { SHRUB } & \square \text { LOCAL SHRUB } \\ \nabla \text { GRASS } & \square \text { LOCAL GRASS } \\ \nabla \text { FORBS } & \square \text { LOCAL FORBS } \\ \nabla \text { WETLAND } & \square \text { LOCAL WETLAND }\end{array}$

URL: none currently available

Tacoma

Weyerhaeuser

WWC $2 F 2$

Tacoma, WA

98477

PHONE: (800) 732-4769

$\begin{array}{ll}\square \text { TREE } & \square \text { LOCAL TREE } \\ \square \text { SHRUB } & \square \text { LOCAL SHRUB } \\ \nabla \text { GRASS } & \square \text { LOCAL GRASS } \\ \nabla \text { FORBS } & \square \text { LOCAL FORBS } \\ \square \text { WETLAND } & \square \text { LOCAL WETLAND }\end{array}$

URL: www.weyerhaeuser.com

\section{Tekoa}

Plants of the Wild

PO Box 866

Tekoa, WA

99033

PHONE: (509) 284-2848

$\begin{array}{ll}\nabla \text { TREE } & \square \text { LOCAL TREE } \\ \square \text { SHRUB } & \square \text { LOCAL SHRUB } \\ \square \text { GRASS } & \square \text { LOCAL GRASS } \\ \square \text { FORBS } & \square \text { LOCAL FORBS } \\ \square \text { WETLAND } & \square \text { LOCAL WETLAND }\end{array}$

URL: www.plantsofthewild.com

\section{Woodinville}

Pacific Natives \& Ornamentals

23423 57th Ave. S.E.

Woodinville, WA

98072

PHONE: (425) 483-8108

$\begin{array}{ll}\nabla \text { TREE } & \square \text { LOCAL TREE } \\ \nabla \text { SHRUB } & \square \text { LOCAL SHRUB } \\ \nabla \text { GRASS } & \square \text { LOCAL GRASS } \\ \nabla \text { FORBS } & \square \text { LOCAL FORBS } \\ \square \text { WETLAND } & \square \text { LOCAL WETLAND }\end{array}$

$\checkmark$ WETLAND $\square$ LOCAL WETLAND

URL: none currently available 


$\begin{array}{lll}\text { Vibrant Plants } & & \\ \text { PO Box } 627 & \square \text { TREE } & \square \text { LOCAL TREE } \\ \text { Woodinville, WA } & \square \text { SHRUB } & \square \text { LOCAL SHRUB } \\ 98072 & \square \text { GRASS } & \square \text { LOCAL GRASS } \\ \text { PHONE: (425) 488-1155 } & \square \text { FORBS } & \square \text { LOCAL FORBS } \\ & \square \text { WETLAND } & \square \text { LOCAL WETLAND }\end{array}$

URL: www.vibrantplants.com

\section{Wisconsin}

Ashland

Deercreek Seed Farm

Box 105

Ashland, WI

54806

PHONE: (715) 278-3200

$\begin{array}{ll}\square \text { TREE } & \square \text { LOCAL TREE } \\ \square \text { SHRUB } & \square \text { LOCAL SHRUB } \\ \square \text { GRASS } & \square \text { LOCAL GRASS } \\ \square \text { FORBS } & \square \text { LOCAL FORBS } \\ \square \text { WETLAND } & \square \text { LOCAL WETLAND }\end{array}$

URL: none currently available

Bayfield

Hausers Superior View Farm

Rt.1, Box 199"

Bayfield, WI

54814

PHONE: (715) 779-5404

$\begin{array}{ll}\square \text { TREE } & \square \text { LOCAL TREE } \\ \square \text { SHRUB } & \square \text { LOCAL SHRUB } \\ \square \text { GRASS } & \square \text { LOCAL GRASS } \\ \square \text { FORBS } & \square \text { LOCAL FORBS } \\ \square \text { WETLAND } & \square \text { LOCAL WETLAND }\end{array}$

URL: wWw.superionviewfarm.com

Brodhead

Applied Ecological Service, Inc. / Taylor Creek Restoration Nursery

$\mathrm{PO}$ Box 256

$\checkmark$ TREE

Brodhead, WI

$\checkmark$ SHRUB

$\nabla$ GRASS

53520

$\nabla$ FORBS

$\square$ WETLAND

$\checkmark$ LOCAL TREE

$\checkmark$ LOCAL SHRUB

$\nabla$ LOCAL GRASS

PHONE: (608) 897-8641

$\checkmark$ LOCAL FORBS

URL: www.appliedeco.com

De Pere

Ambrosius Sales \& Service

$840 \mathrm{~N}$ 9th St

De Pere, WI

54115

PHONE: (920) 336-4575

$\begin{array}{ll}\square \text { TREE } & \square \text { LOCAL TREE } \\ \square \text { SHRUB } & \square \text { LOCAL SHRUB } \\ \square \text { GRASS } & \square \text { LOCAL GRASS } \\ \square \text { FORBS } & \square \text { LOCAL FORBS } \\ \square \text { WETLAND } & \square \text { LOCAL WETLAND }\end{array}$

URL: www.ambrosiussas.com

Progressive Farmers $\mathrm{C}_{0}$-op

1221 Grant St.

De Pere, WI

54115

PHONE: (920) 336-8921

$\begin{array}{ll}\square \text { TREE } & \square \text { LOCAL TREE } \\ \square \text { SHRUB } & \square \text { LOCAL SHRUB } \\ \square \text { GRASS } & \square \text { LOCAL GRASS } \\ \square \text { FORBS } & \square \text { LOCAL FORBS } \\ \square \text { WETLAND } & \square \text { LOCAL WETLAND }\end{array}$

URL: www.progressivefarmers.com 
Hayward

Chief River Nursery

10681 N McClain Rd

Hayward, WI

54843

PHONE: (800) 367-9254

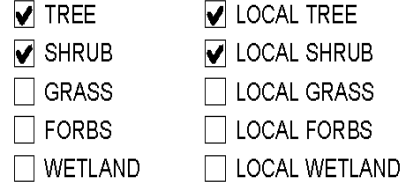

URL: http://www.walinursery.com/

Hixton

Toddle-In Nursery

Rt.2, Box 58"

Hixton, WI

54635

$\square$ LOCAL SHRUB

PHONE: (715) 963-3561

$\square$ FORBS $\quad \square$ LOCAL FORBS

$\square$ WETLAND $\square$ LOCAL WETLAND

URL: none currently available

\begin{tabular}{|c|c|c|}
\hline \multicolumn{3}{|l|}{ Junction City } \\
\hline \multicolumn{3}{|l|}{ FS Co-op } \\
\hline 625 Main St & $\square$ TREE & $\square$ LOCAL TREE \\
\hline Junction City, WI & $\square$ SHRUB & $\square$ LOCAL SHRUB \\
\hline 54443 & $\nabla$ GRASS & $\square$ LOCAL GRASS \\
\hline PHONE: (715) 457-2311 & $\checkmark$ FORBS & $\square$ LOCAL FORBS \\
\hline & $\square$ WETLAND & $\square$ LOCAL WETLAND \\
\hline
\end{tabular}

URL: none currently available

\section{Lacrosse}

LaCrosse Forage \& Tur

2615 Commerce St.

Lacrosse, WI

54602

PHONE: (608) 783-9560

$\begin{array}{ll}\square \text { TREE } & \square \text { LOCAL TREE } \\ \square \text { SHRUB } & \square \text { LOCAL SHRUB } \\ \square \text { GRASS } & \square \text { LOCAL GRASS } \\ \square \text { FORBS } & \square \text { LOCAL FORBS } \\ \square \text { WETLAND } & \square \text { LOCAL WETLAND }\end{array}$

URL: www.lftseed.com

Wisconsin DNR / State Forest Nursery

$\begin{array}{lll}3550 \text { Mormon Coulee Road } & \square \text { TREE } & \square \text { LOCAL TREE } \\ \text { Lacrosse, WI } & \square \text { SHRUB } & \square \text { LOCAL SHRUB } \\ 54601 & \square \text { GRASS } & \square \text { LOCAL GRASS } \\ \text { PHONE: (608) 785-9011 } & \square \text { FORBS } & \square \text { LOCAL FORBS } \\ & \square \text { WETLAND } & \square \text { LOCAL WETLAND }\end{array}$

URL: http:/Wwww.dnr.state.wi.us/orgland/forestry/Nurseryindex.htm

\section{Madison}

Olds Seed Solutions

2901 Packers Ave

Madison, WI

53707

PHONE: (800) 356-7333

$\begin{array}{ll}\square \text { TREE } & \square \text { LOCAL TREE } \\ \square \text { SHRUB } & \square \text { LOCAL SHRUB } \\ \nabla \text { GRASS } & \square \text { LOCAL GRASS } \\ \nabla \text { FORBS } & \square \text { LOCAL FORBS } \\ \square \text { WETLAND } & \square \text { LOCAL WETLAND }\end{array}$

URL: www.seedsolutions.com 
Manawa

Tomorrow Valley Coop

961 Depot St

Manawa, WI

54949

PHONE: (877) 596-8827

$\begin{array}{ll}\square \text { TREE } & \square \text { LOCAL TREE } \\ \square \text { SHRUB } & \square \text { LOCAL SHRUB } \\ \square \text { GRASS } & \square \text { LOCAL GRASS } \\ \square \text { FORBS } & \square \text { LOCAL FORBS } \\ \square \text { WETLAND } & \square \text { LOCAL WETLAND }\end{array}$

URL: www.tomorrowvalley.com

Mason

Nel son Seed Farm

Rt.1, Box $197 \mathrm{~A}^{\prime \prime}$

Mason, WI

54856

$\square$ TREE

$\square$ SHRUB

$\square$ LOCAL TREE

$\square$ GRASS

$\square$ LOCAL SHRUB

PHONE: (715) 765-4591

$\checkmark$ FORBS

$\square$ LOCAL GRASS

$\square$ WETLAND $\square$ LOCAL WETLAND

URL: none currently available

Middleton

Bruce Miller Nursery

$\begin{array}{lll}2830 \text { West Beltline Highway } & \nabla \text { TREE } & \square \text { LOCAL TREE } \\ \text { Middleton, WI } & \square \text { SHRUB } & \square \text { LOCAL SHRUB } \\ 53562 & \square \text { GRASS } & \square \text { LOCAL GRASS } \\ \text { PHONE: (608) 836-7041 } & \square \text { FORBS } & \square \text { LOCAL FORBS } \\ & \square \text { WETLAND } & \square \text { LOCAL WETLAND }\end{array}$

URL: www.bruceco.com

\section{Mt. Horeb}

Prairie Ridge Nursery

9738 Overland Rd.

Mt. Horeb, WI

53572

PHONE: (608) 437-5245

$\begin{array}{ll}\square \text { TREE } & \square \text { LOCAL TREE } \\ \square \text { SHRUB } & \square \text { LOCAL SHRUB } \\ \square \text { GRASS } & \square \text { LOCAL GRASS } \\ \nabla \text { FORBS } & \square \text { LOCAL FORBS } \\ \square \text { WETLAND } & \square \text { LOCAL WETLAND }\end{array}$

URL: www.prairieridgenursery.com

\section{Neshkoro}

Rohdes Nursery

N8098 Duck Creek Ave

Neshkoro, WI

54960

PHONE: (920) 293-4374

$\begin{array}{ll}\nabla \text { TREE } & \square \text { LOCAL TREE } \\ \square \text { SHRUB } & \square \text { LOCAL SHRUB } \\ \nabla \text { GRASS } & \square \text { LOCAL GRASS } \\ \nabla \text { FORBS } & \square \text { LOCAL FORBS } \\ \square \text { WETLAND } & \square \text { LOCAL WETLAND }\end{array}$

URL: none currently available

\section{New Richmond}

Blue Ribbon Seed \& Garden Ctr. 513 N. Knowles Ave.

New Richmond, WI

54017

PHONE: (715) 246-2902

$\begin{array}{ll}\square \text { TREE } & \square \text { LOCAL TREE } \\ \square \text { SHRUB } & \square \text { LOCAL SHRUB } \\ \square \text { GRASS } & \square \text { LOCAL GRASS } \\ \square \text { FORBS } & \square \text { LOCAL FORBS } \\ \square \text { WETLAND } & \square \text { LOCAL WETLAND }\end{array}$

URL: none currently available 
Omro

Kesters Wild Game Food, Inc.

PO Box 516

Omro, WI

54963

PHONE: (920) 685-2929

$\begin{array}{ll}\square \text { TREE } & \square \text { LOCAL TREE } \\ \square \text { SHRUB } & \square \text { LOCAL SHRUB } \\ \square \text { GRASS } & \square \text { LOCAL GRASS } \\ \square \text { FORBS } & \square \text { LOCAL FORBS } \\ \square \text { WETLAND } & \square \text { LOCAL WETLAND }\end{array}$

URL: www.kestersnursery.com

\section{Oshkosh}

Wildlife Nurseries, Inc.

PO Box 2724

Oshkosh, WI

54903

$\square$ SHRUB $\quad \square$ LOCAL SHRUB

PHONE: (920) 231-3780

$\nabla$ GRASS $\square$ LOCAL GRASS

$\begin{array}{ll}\square \text { FORBS } & \square \text { LOCAL FORBS } \\ \square \text { WETLAND } & \square \text { LOCAL WETLAND }\end{array}$

URL: none currently available

Osseo

KF Evergreens

N 12757 Rindahl Valley Rd

Osseo, WI

54758

PHONE: (800) 423-3530

URL: www.kfevergreens.com

Plainfield

Lauras Lane Nursery

Box 232

$\checkmark$ TREE $\quad \nabla$ LOCAL TREE

Plainfield, WI

54966

$\checkmark$ SHRUB

$\checkmark$ LOCAL SHRUB

PHONE: (715) 366-2477

$\square$ GRASS $\square$ LOCAL GRASS

$\square$ FORBS $\square$ LOCAL FORBS

$\square$ WETLAND $\square$ LOCAL WETLAND

URL: none currently available

Racine

Mileagers Gardens

4838 Douglas Ave.

Racine, W|

53402

PHONE: (800) 669-1229

$\begin{array}{ll}\square \text { TREE } & \square \text { LOCAL TREE } \\ \square \text { SHRUB } & \square \text { LOCAL SHRUB } \\ \square \text { GRASS } & \square \text { LOCAL GRASS } \\ \square \text { FORBS } & \square \text { LOCAL FORBS } \\ \square \text { WETLAND } & \square \text { LOCAL WETLAND }\end{array}$

URL: Www.milaegers.com

\section{Randolph}

J.W. Jung Seed Co

335 S. High St.

Randolph, W

53957

PHONE: (800) 297-3123

$\begin{array}{ll}\nabla \text { TREE } & \square \text { LOCAL TREE } \\ \nabla \text { SHRUB } & \square \text { LOCAL SHRUB } \\ \square \text { GRASS } & \square \text { LOCAL GRASS } \\ \square \text { FORBS } & \square \text { LOCAL FORBS } \\ \square \text { WETLAND } & \square \text { LOCAL WETLAND }\end{array}$

URL: www.jungseed.com 


\section{South Milwauke}

Honadels, Inc.

1204 Minnesota Ave.

South Milwaukee, WI

53172

PHONE: (414) 762-0458

URL: none currently available

\section{Sturgeon Bay}

Evergreen Nursery

5027 County TT

Sturgeon Bay, WI

54235

PHONE: (800) 448-5691

URL: www.evergreennurseryco.com

$\begin{array}{ll}\square \text { TREE } & \square \text { LOCAL TREE } \\ \square \text { SHRUB } & \square \text { LOCAL SHRUB } \\ \square \text { GRASS } & \square \text { LOCAL GRASS } \\ \square \text { FORBS } & \square \text { LOCAL FORBS } \\ \square \text { WETLAND } & \square \text { LOCAL WETLAND }\end{array}$

$\square$ WETLAND $\square$ LOCAL WETLAND

$x$

Prairie Future Seed Co.

P.O. Box 644

Sussex, WI

53089

PHONE: (262) 820-0221

$\begin{array}{ll}\nabla \text { TREE } & \nabla \text { LOCAL TREE } \\ \square \text { SHRUB } & \nabla \text { LOCAL SHRUB } \\ \square \text { GRASS } & \square \text { LOCAL GRASS } \\ \nabla \text { FORBS } & \square \text { LOCAL FORBS } \\ \square \text { WETLAND } & \square \text { LOCAL WETLAND }\end{array}$

$\square$ WETLAND

$\square$ LOCAL WETLAND

URL: none currently available

Waterloo

McKay Nursery $\mathrm{C}_{0}$

PO Box 185

Waterloo, WI

53594

PHONE: (920) 478-2121

$\begin{array}{ll}\square \text { TREE } & \square \text { LOCAL TREE } \\ \square \text { SHRUB } & \square \text { LOCAL SHRUB } \\ \nabla \text { GRASS } & \nabla \text { LOCAL GRASS } \\ \nabla \text { FORBS } & \square \text { LOCAL FORBS } \\ \square \text { WETLAND } & \square \text { LOCAL WETLAND }\end{array}$

URL: www.mckaynursery.com

Waupaca

Jay-Mlar Inc.

905 Ware St.

Waupaca, WI

54981

PHONE: (715) 258-3234

$\begin{array}{ll}\square \text { TREE } & \square \text { LOCAL TREE } \\ \nabla \text { SHRUB } & \square \text { LOCAL SHRUB } \\ \square \text { GRASS } & \square \text { LOCAL GRASS } \\ \nabla \text { FORBS } & \square \text { LOCAL FORBS } \\ \square \text { WETLAND } & \square \text { LOCAL WETLAND }\end{array}$

$\checkmark$ WETLAND $\square$ LOCAL WETLAND

URL: none currently available

\section{Wautoma}

Campbell Tree \& Land $\mathrm{Co}$.

W7427 State Rd 21 and 73

Wautoma, WI

54982

PHONE: (920) 787-4653

$\begin{array}{ll}\square \text { TREE } & \square \text { LOCAL TREE } \\ \square \text { SHRUB } & \square \text { LOCAL SHRUB } \\ \square \text { GRASS } & \square \text { LOCAL GRASS } \\ \square \text { FORBS } & \square \text { LOCAL FORBS } \\ \square \text { WETLAND } & \square \text { LOCAL WETLAND }\end{array}$

$\begin{array}{ll}\square \text { TREE } & \square \text { LOCAL TREE } \\ \nabla \text { SHRUB } & \square \text { LOCAL SHRUB } \\ \square \text { GRASS } & \square \text { LOCAL GRASS } \\ \square \text { FORBS } & \square \text { LOCAL FORBS } \\ \square \text { WETLAND } & \square \text { LOCAL WETLAND }\end{array}$

URL: none currently available 
Westfield

Prairie Nursery

Box 306

Westield, WI

53964

PHONE: (800) 476-9453

$\begin{array}{ll}\square \text { TREE } & \square \text { LOCAL TREE } \\ \square \text { SHRUB } & \square \text { LOCAL SHRUB } \\ \nabla \text { GRASS } & \square \text { LOCAL GRASS } \\ \nabla \text { FORBS } & \square \text { LOCAL FORBS } \\ \nabla \text { WETLAND } & \square \text { LOCAL WETLAND }\end{array}$

URL: www.prairienursery.com

White Lake

Wolf River Valley Seeds

\section{N2976 Hwy M}

White Lake, WI

54491

PHONE: (800) 359-2480

$\begin{array}{ll}\square \text { TREE } & \square \text { LOCAL TREE } \\ \square \text { SHRUB } & \square \text { LOCAL SHRUB } \\ \square \text { GRASS } & \square \text { LOCAL GRASS } \\ \square \text { FORBS } & \square \text { LOCAL FORBS } \\ \square \text { WETLAND } & \square \text { LOCAL WETLAND }\end{array}$

URL: www.wolfrivervalleyseeds.com

Wild Rose

J \& J Transplant Aquatic Nursery, Inc.

$\begin{array}{lll}\text { PO Box } 227 & \square \text { TREE } & \square \text { LOCAL TREE } \\ \text { Wild Rose, WI } & \square \text { SHRUB } & \square \text { LOCAL SHRUB } \\ 54984 & \square \text { GRASS } & \square \text { LOCAL GRASS } \\ \text { PHONE: }(800) \text { 622-5055 } & \square \text { FORBS } & \square \text { LOCAL FORBS } \\ & \square \text { WETLAND } & \square \text { LOCAL WETLAND }\end{array}$

URL: www.tranzplant.com

\section{West Virginia}

Alderson

Plant Mlaterials Center

PO Box 390

Alderson, W

24910

PHONE: (304) 445-3005

$\begin{array}{ll}\square \text { TREE } & \square \text { LOCAL TREE } \\ \square \text { SHRUB } & \square \text { LOCAL SHRUB } \\ \square \text { GRASS } & \square \text { LOCAL GRASS } \\ \square \text { FORBS } & \square \text { LOCAL FORBS } \\ \square \text { WETLAND } & \square \text { LOCAL WETLAND }\end{array}$

URL: www.plant-materials.nrcs.usda.gov/wvpmc/

Nitro

JMD Company

160 West 19th Street

Nitro, W

25143

PHONE: (304) 755-2800

$\begin{array}{ll}\square \text { TREE } & \square \text { LOCAL TREE } \\ \square \text { SHRUB } & \square \text { LOCAL SHRUB } \\ \square \text { GRASS } & \square \text { LOCAL GRASS } \\ \square \text { FORBS } & \square \text { LOCAL FORBS } \\ \square \text { WETLAND } & \square \text { LOCAL WETLAND }\end{array}$

URL: www.mdcompany.com

Renick

Sunshine Farm \& Garden

HC 67, Box 539B

Renick, WW

24966

PHONE: (304) 497-2208

$\begin{array}{ll}\nabla \text { TREE } & \square \text { LOCAL TREE } \\ \nabla \text { SHRUB } & \square \text { LOCAL SHRUB } \\ \nabla \text { GRASS } & \square \text { LOCAL GRASS } \\ \nabla \text { FORBS } & \square \text { LOCAL FORBS } \\ \square \text { WETLAND } & \square \text { LOCAL WETLAND }\end{array}$

URL: http:/Nww'sunfarm.com/ 
Romney

Hampshire Co-op

56 S. Marsham St.

Romney, WV

26757

PHONE: (304) 822-5115

URL: none currently available

\section{West Columbia}

Clements State Tree Nursery

PO Box 8

West Columbia, WW

25287

PHONE: (304) 675-1820

$\begin{array}{ll}\square \text { TREE } & \square \text { LOCAL TREE } \\ \square \text { SHRUB } & \square \text { LOCAL SHRUB } \\ \square \text { GRASS } & \square \text { LOCAL GRASS } \\ \square \text { FORBS } & \square \text { LOCAL FORBS } \\ \square \text { WETLAND } & \square \text { LOCAL WETLAND }\end{array}$

$\square$ WETLAND

$\begin{array}{ll}\nabla \text { TREE } & \square \text { LOCAL TREE } \\ \nabla \text { SHRUB } & \nabla \text { LOCAL SHRUB } \\ \square \text { GRASS } & \square \text { LOCAL GRASS } \\ \square \text { FORBS } & \square \text { LOCAL FORBS } \\ \square \text { WETLAND } & \square \text { LOCAL WETLAND }\end{array}$

URL: http://www.wworestry.com/nursery.cfm?menucall=nurse

\section{Wyoming}

Buffalo

Johnson County $\mathrm{C}_{0}-\mathrm{op}$

Box 907

Buffalo, WY

82834

PHONE: (307) 684-9561

URL: none currently available

Casper

Noland Feed

268 Industrial Ave.

Casper, WY

82601

$\begin{array}{ll}\square \text { TREE } & \square \text { LOCAL TREE } \\ \square \text { SHRUB } & \square \text { LOCAL SHRUB } \\ \square \text { GRASS } & \square \text { LOCAL GRASS } \\ \square \text { FORBS } & \square \text { LOCAL FORBS } \\ \square \text { WETLAND } & \square \text { LOCAL WETLAND }\end{array}$

PHONE: (307) 237-8926

$\begin{array}{ll}\square \text { TREE } & \square \text { LOCAL TREE } \\ \square \text { SHRUB } & \square \text { LOCAL SHRUB } \\ \square \text { GRASS } & \square \text { LOCAL GRASS } \\ \square \text { FORBS } & \square \text { LOCAL FORBS } \\ \square \text { WETLAND } & \square \text { LOCAL WETLAND }\end{array}$

URL: none currently available

Gillette

Farmers Co-op

207 E. 1st St.

Gillette, WY

82716

PHONE: (307) 682-4772

$\begin{array}{ll}\square \text { TREE } & \square \text { LOCAL TREE } \\ \square \text { SHRUB } & \square \text { LOCAL SHRUB } \\ \square \text { GRASS } & \square \text { LOCAL GRASS } \\ \square \text { FORBS } & \square \text { LOCAL FORBS } \\ \square \text { WETLAND } & \square \text { LOCAL WETLAND }\end{array}$

URL: none currently available

Thars Feed \& Ranch Supply

100 S. Burma Ave.

Gillette, WY

82716

PHONE: (307) 682-9501

$\begin{array}{ll}\square \text { TREE } & \square \text { LOCAL TREE } \\ \square \text { SHRUB } & \square \text { LOCAL SHRUB } \\ \square \text { GRASS } & \square \text { LOCAL GRASS } \\ \square \text { FORBS } & \square \text { LOCAL FORBS } \\ \square \text { WETLAND } & \square \text { LOCAL WETLAND }\end{array}$

URL: none currently available 
Manderson

Wind River Seed

3075 Lane 51 1/2

Manderson. WY

82432

TREE

$\checkmark$ LOCAL TREE

PHONE: (307) 568-3361

$\square$ SHRUB

$\checkmark$ GRASS

$\square$ LOCAL SHRUB

$\checkmark$ LOCAL GRASS

$\square$ WETLAND $\square$ LOCAL WETLAND

URL: www.windriverseed.com

Powell

Etheridge Seed Farm

1950 Lane 11

Powell, WY

82435

$\square$ SHRUB $\quad \square$ LOCAL SHRUB

$\checkmark$ GRASS $\square$ LOCAL GRASS

PHONE: (307) 754-2371

$\begin{array}{ll}\square \text { FORBS } & \square \text { LOCAL FORBS } \\ \square \text { WETLAND } & \square \text { LOCAL WETLAND }\end{array}$

URL: none currently available

Mike Forman

990 Rd. 19

Powell, WY

82435

PHONE: (307) 754-5360

$\begin{array}{ll}\square \text { TREE } & \square \text { LOCAL TREE } \\ \square \text { SHRUB } & \square \text { LOCAL SHRUB } \\ \square \text { GRASS } & \square \text { LOCAL GRASS } \\ \square \text { FORBS } & \square \text { LOCAL FORBS } \\ \square \text { WETLAND } & \square \text { LOCAL WETLAND }\end{array}$

URL: none currently available

Spiering Farms

1170 Rd. 19

Powell, WY

82435

PHONE: (307) 754-4349

$\begin{array}{ll}\square \text { TREE } & \square \text { LOCAL TREE } \\ \square \text { SHRUB } & \square \text { LOCAL SHRUB } \\ \square \text { GRASS } & \square \text { LOCAL GRASS } \\ \square \text { FORBS } & \square \text { LOCAL FORBS } \\ \square \text { WETLAND } & \square \text { LOCAL WETLAND }\end{array}$

URL: none currently available

\section{Sheridan}

Sheridan Seed \& Supply

161 N. Scott St.

Sheridan, WY

82801

PHONE: (307) 672-2475

$\begin{array}{ll}\square \text { TREE } & \square \text { LOCAL TREE } \\ \square \text { SHRUB } & \square \text { LOCAL SHRUB } \\ \square \text { GRASS } & \square \text { LOCAL GRASS } \\ \square \text { FORBS } & \square \text { LOCAL FORBS } \\ \square \text { WETLAND } & \square \text { LOCAL WETLAND }\end{array}$

URL: none currently available

\section{Torrington}

Z\&W Mill

Box 236

Torrington, WY

82240

PHONE: (307) 532-2347

$\begin{array}{ll}\square \text { TREE } & \square \text { LOCAL TREE } \\ \square \text { SHRUB } & \square \text { LOCAL SHRUB } \\ \square \text { GRASS } & \square \text { LOCAL GRASS } \\ \square \text { FORBS } & \square \text { LOCAL FORBS } \\ \square \text { WETLAND } & \square \text { LOCAL WETLAND }\end{array}$

URL: none currently available 


\section{Conclusion and Recommendation}

\section{Conclusion}

This research has created a comprehensive, up-to-date listing of plant material vendors. The list includes vendors of trees, shrubs, grasses, wild-flowers, and wetland plants.

\section{Recommendation}

Plant material vendors, like all seasonal, agricultural businesses, are subject to changes in weather and market shifts. It is reasonable to assume a number of the businesses listed here will leave the market, and many new businesses will take their places. It is recommended that this listing be updated and verified annually, and that current listings be made available via the World-Wide Web. 


\section{Bibliography}

“1994 Buyers Guide,” Land and Water 37(November/December):32-80.

Harker, D., S. Evans, M. Evans, and K. Harker, Landscape Restoration Handbook (Lewis Publishers, Boca Raton, FL, 1993).

International Erosion Control Association, 1993-1994 Products and Services Directory (Steamboat Springs, CO, 1993).

Soil and Water Conservation Society, Sources of Native Seeds and Plants (Ankeny, IA, 1087).

Soil Conservation Service, Directory of Wetland Plant Vendors, U.S. Army Corps of Engineers, Waterways Experiment Station, Wetlands Research Program Technical Report WRP-SM1. 


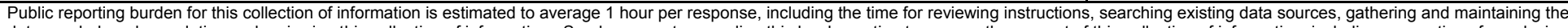

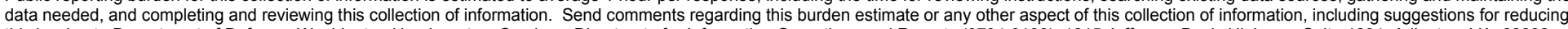

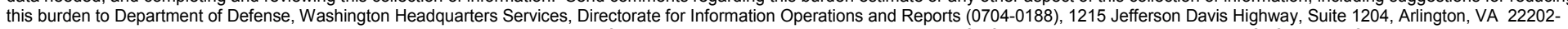

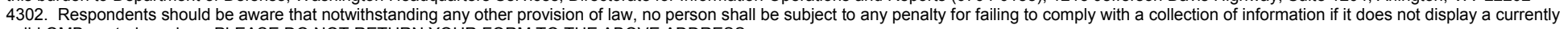
valid OMB control number. PLEASE DO NOT RETURN YOUR FORM TO THE ABOVE ADDRESS.
1. REPORT DATE (DD-MM-YYYY)
2. REPORT TYPE
3. DATES COVERED (From - To)

$12-2004$

Final

4. TITLE AND SUBTITLE

Sources of Plant Materials for Land Rehabilitation

5a. CONTRACT NUMBER

5b. GRANT NUMBER

5c. PROGRAM ELEMENT NUMBER

6. AUTHOR(S)

Heidi R. Howard, John A. Guretzky, Brandy S. Koch, Shannon N. McDowell, and Steven D.

Warren

5d. PROJECT NUMBER

MIPR

5e. TASK NUMBER

5f. WORK UNIT NUMBER

$134 \mathrm{GH} 5$

8. PERFORMING ORGANIZATION REPORT NUMBER

ERDC/CERL SR-04-34

Construction Engineering Research Laboratory (CERL)

PO Box 9005

Champaign, IL 61826-9005

9. SPONSORING / MONITORING AGENCY NAME(S) AND ADDRESS(ES)

U.S. Army Environmental Center (USAEC)

Pollution Prevention and Environmental

Technology Division

Aberdeen Proving Ground, MD 21010-5401

Colorado State University

Center for Environmental Management of

Military Lands

1490 Campus Delivery

Fort Collins, CO 80523-1490

10. SPONSOR/MONITOR'S ACRONYM(S)

11. SPONSOR/MONITOR'S REPORT NUMBER(S)

\section{DISTRIBUTION / AVAILABILITY STATEMENT}

Approved for public release; distribution is unlimited.

\section{SUPPLEMENTARY NOTES}

Copies are available from the National Technical Information Service, 5285 Port Royal Road, Springfield, VA 22161.

\section{ABSTRACT}

Military training often results in environmental degradation that has negative impacts on future training missions. The U.S. Army Construction Engineering Research Laboratory (CERL) conceptualized the Army's Integrated Training Area Management (ITAM) program in response to the concerns expressed by military land managers and trainers over the declining condition of training and testing lands. ITAM has evolved to include land condition and trend analysis (LCTA), environmental awareness (EA), training requirements integration (TRI), and land rehabilitation and maintenance (LRAM). The need for LRAM has increased dramatically over the past decade. Among the many difficul-ties faced in planning and implementing a land rehabilitation project is procurement of appropriate plant materials for revegetation of damaged lands. To satisfy government procurement requirements and ensure a fair and reasonable price for materials, it is usually necessary to identify and solicit bids from multiple vendors. Moreover, there is an increasing emphasis on the use of locally endemic or adapted plant species. These factors increase the demand to identify multiple commercial sources of regional or local sources of plant materials. This research compiled a comprehensive, current list of plant material vendors, including vendors of trees, shrubs, grasses, wild-flowers, and wetland plants.

\section{SUBJECT TERMS}

land management plant material

rehabilitation

natural resource management

\begin{tabular}{|c|c|c|c|c|c|}
\hline \multicolumn{3}{|c|}{ 16. SECURITY CLASSIFICATION OF: } & \multirow{2}{*}{$\begin{array}{l}\text { 17. LIMITATION } \\
\text { OF ABSTRACT } \\
\text { SAR }\end{array}$} & \multirow{2}{*}{$\begin{array}{l}\text { 18. NUMBER } \\
\text { OF PAGES } \\
212\end{array}$} & \multirow{2}{*}{$\begin{array}{l}\text { 19a. NAME OF RESPONSIBLE PERSON } \\
\text { Heidi R. Howard } \\
\text { 19b. TELEPHONE NUMBER (in- } \\
\text { clude area code) } \\
\text { (217) } 352-6511, \mathrm{x}-7601\end{array}$} \\
\hline $\begin{array}{l}\text { a. REPORT } \\
\text { Unclassified }\end{array}$ & $\begin{array}{l}\text { b. ABSTRACT } \\
\text { Unclassified }\end{array}$ & $\begin{array}{l}\text { c. THIS PAGE } \\
\text { Unclassified }\end{array}$ & & & \\
\hline
\end{tabular}

